\title{
DEVELOPMENT OF AN AIR-CYCLE ENVIRONMENTAL CONTROL SYSTEM FOR AUTOMOTIVE
} APPLICATIONS

\author{
A thesis \\ Presented to \\ the faculty of California Polytechnic State University \\ San Luis Obispo \\ In Partial Fulfillment of \\ the Requirements for the Degree of \\ Master of Science in Mechanical Engineering
}

by

Christopher J. Forster

December 2009 
(C) 2009

Christopher J. Forster

ALL RIGHTS RESERVED 
APPROVAL PAGE

TITLE: DEVELOPMENT OF AN AIR-CYCLE ENVIRONMENTAL CONTROL SYSTEM FOR AUTOMOTIVE APPLICATIONS

AUTHOR: Christopher James Forster

DATE SUBMITTED:

Committee Chair: Dr. Patrick Lemieux

Committee Member: Dr. Chris Pascual

Committee Member: Dr. Kim Shollenberger 


\begin{abstract}
Development of an Air-Cycle Environmental Control System for Automotive Applications Christopher James Forster

Mechanical Engineering Department

California Polytechnic State University, San Luis Obispo
\end{abstract}

An air-cycle air conditioning system, using a typical automotive turbocharger as the core of the system, was designed and tested. Effects on engine performance were kept to a minimum while providing the maximum amount of cooling possible and minimizing weight and space

requirements. A test stand utilizing shop compressed air was developed to measure component performance. An unmodified automotive turbocharger was tested initially as a baseline in a Reversed-Brayton Cycle air cooling system. Once the baseline was established, another aircycle machine, assembled from commercial turbocharger components chosen individually to optimize their performance for cooling purposes, was tested to improve the overall cycle efficiency. Finally, once the air-cycle air conditioning system was optimized, it was tested on an engine to simulate more realistic operating conditions and performance. The shop-air test stand experiments showed a peak dry-air-rated (DAR) coefficient of performance (COP) of 0.38 and a DAR cooling capacity of 0.45 tons for the baseline turbocharger, and a peak DAR COP of 0.73 and DAR cooling capacity of 1.5 tons for the optimized system with a modified turbocharger. The on-engine testing was limited due to a thrust bearing failure in the ACM. However, the data collected at lower engine load and speed indicates a DAR COP of 0.56 and a DAR cooling capacity of 0.72 tons. On-engine testing was planned to include operating points where the stock turbocharger was utilizing turbine-bypass to limit boost pressure. While it wasn't possible to continue testing, it is expected that DAR COP and cooling capacity would have increased at higher engine load and speed, where turbine-bypass operation typically occurs. 


\section{Acknowledgements}

I would like to thank Dr. Patrick Lemieux for his support and keeping me on task throughout this

project. I would also like to thank Dr. Glen Thorncroft and Jim Gerhardt for their help with instrumentation and lab equipment troubleshooting. 


\section{Table of Contents}

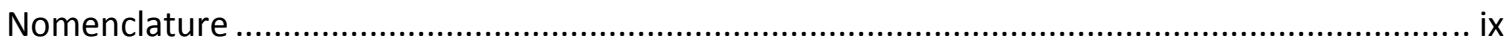

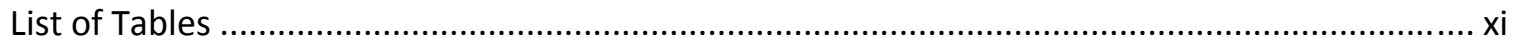

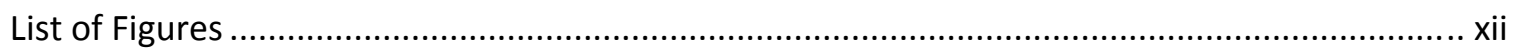

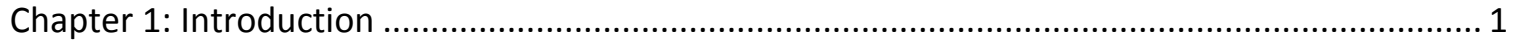

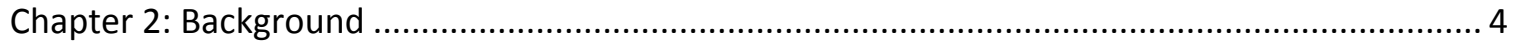

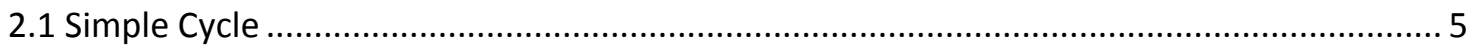

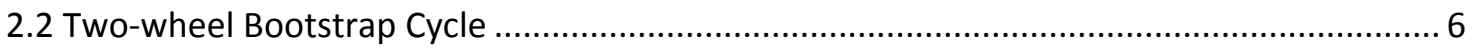

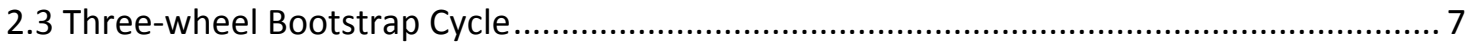

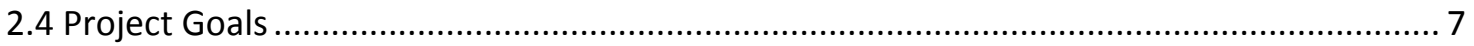

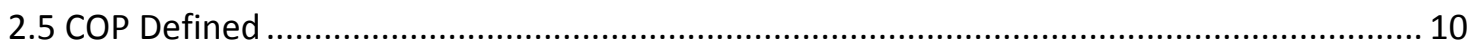

2.6 Previous work performed at Queen's University, Belfast................................................. 11

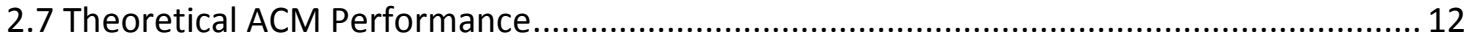

Chapter 3 Experimental Apparatus, Procedures, and Conditions .............................................. 19

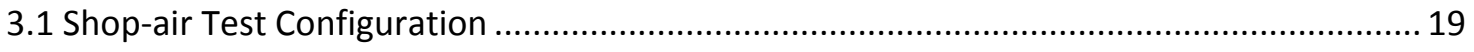

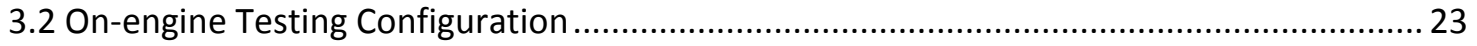

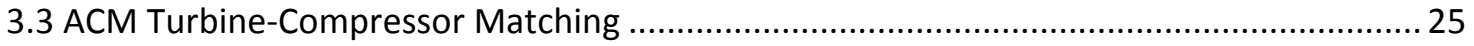

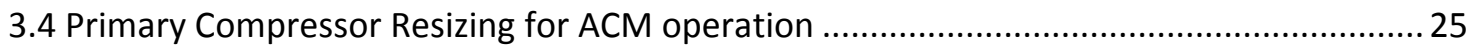

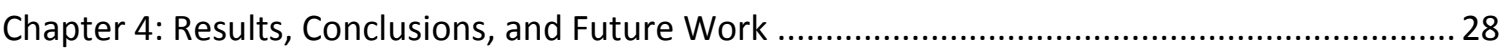

4.1 Results and Discussion from Unmodified GT1241 Shop Air Test Stand ............................28

4.2 Results and Discussion from Modified GT1244 Shop Air Test Stand................................. 31

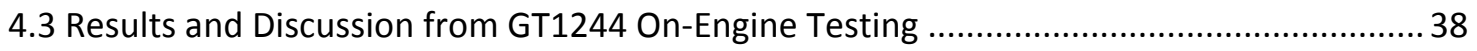

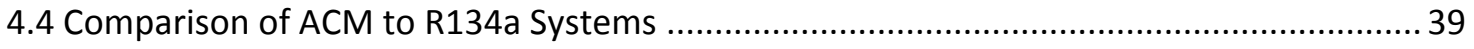

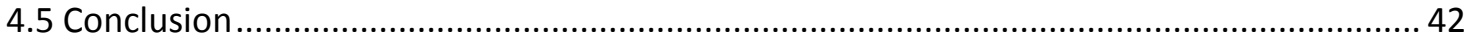

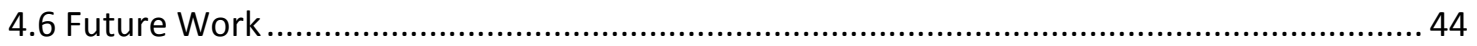

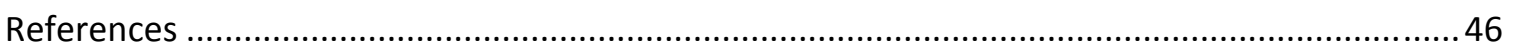

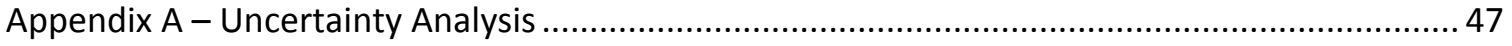

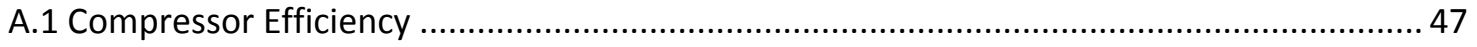

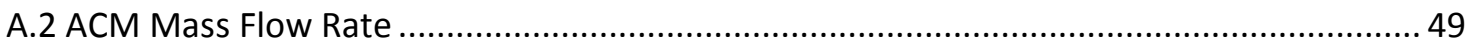




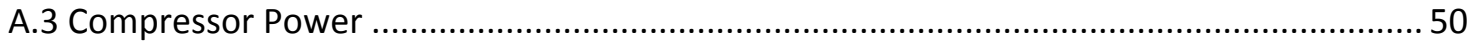

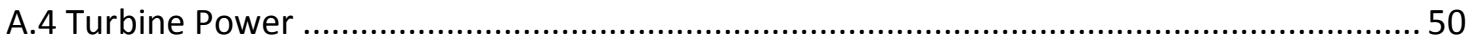

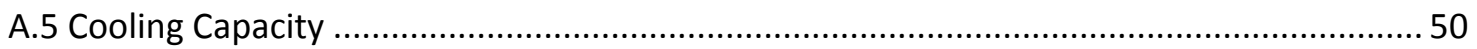

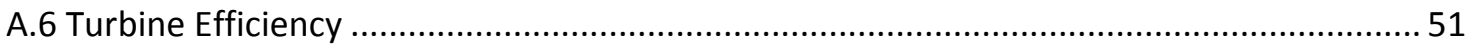

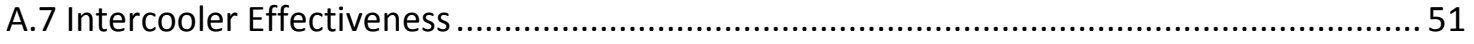

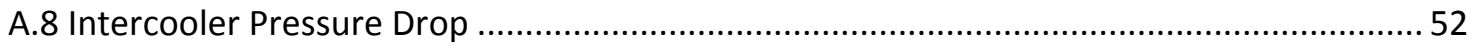

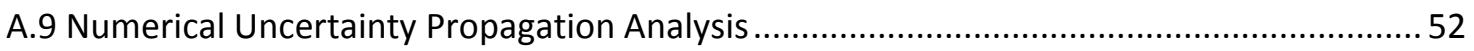

Appendix B - Derivation of Equations for Air-cycle Analysis ....................................................... 53

B.1 Isentropic Compressor Power Requirement .............................................................. 53

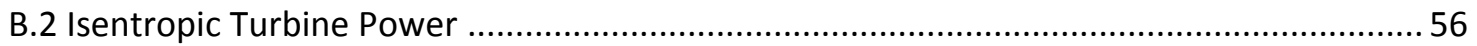

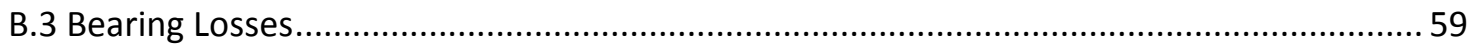

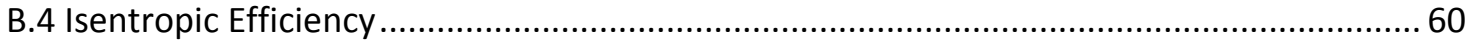

B.4.1 Compressor Isentropic Efficiency Measurement............................................................ 60

B.4.2 Turbine Isentropic Efficiency Measurement with Bearing Losses Combined ...............60

B.4.3 Turbine Isentropic Efficiency Measurement without Bearing Losses Combined.........6 61

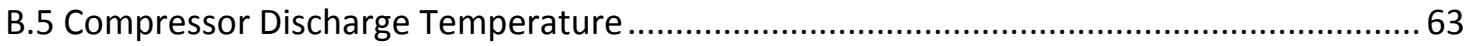

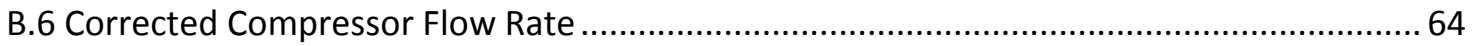

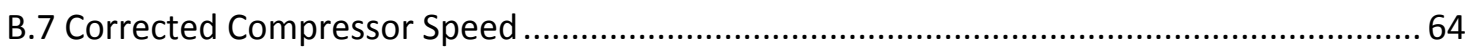

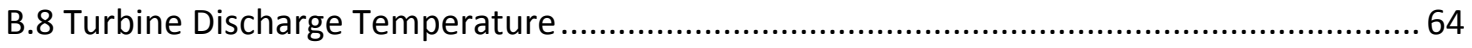

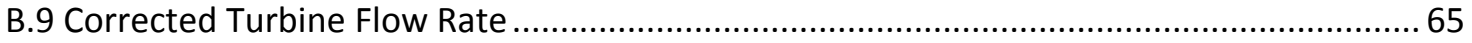

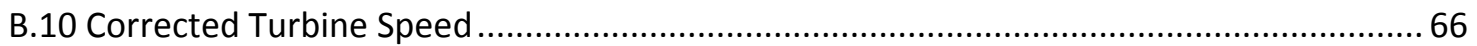

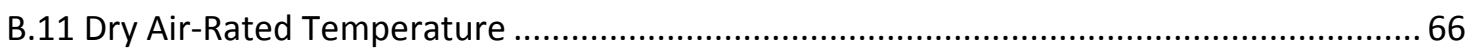

Appendix C - Compressor-Turbine Matching Process …............................................................ 66

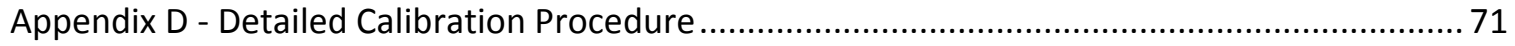

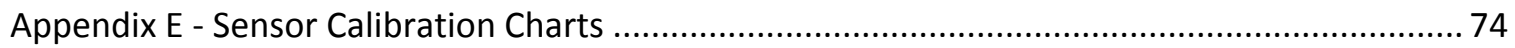

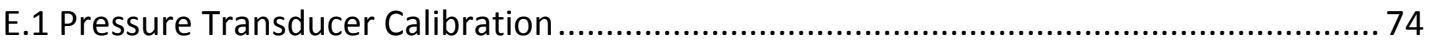

E.2 Laminar Flow Element and Differential Pressure Transducer Calibration ........................ 75

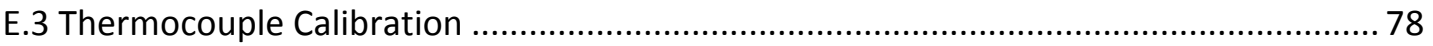

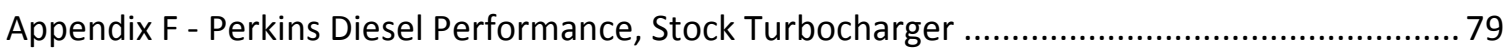

Appendix G - Perkins Diesel Performance, VNT Turbocharger ................................................... 83

Appendix H - Sanden R134a Compressor Performance Chart................................................... 87

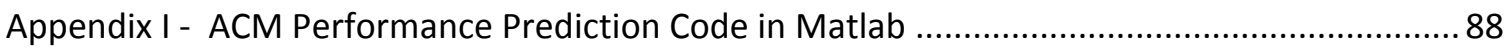


Appendix J - ACM Performance Prediction Code in EES

Appendix K - ACM Single-Point Performance Comparison in EES 147

Appendix L - ACM Component Efficiency Variation Simulation ..... 150

Appendix M - ACM Post-processing Code in Matlab ............................................................. 153

Appendix N - Perkins Diesel Post-processing Code in Matlab .................................................. 175

Appendix O - Perkins Post-processing Comparison in Matlab................................................. 213 


\section{Nomenclature}

$\begin{array}{ll}\mathrm{P}_{1 \mathrm{C}} & \text { Compressor inlet pressure } \\ \mathrm{P}_{2 \mathrm{C}} & \text { Compressor discharge pressure } \\ \mathrm{P}_{1 \mathrm{~T}} & \text { Turbine inlet pressure } \\ \mathrm{P}_{2 \mathrm{~T}} & \text { Turbine discharge pressure } \\ \mathrm{T}_{1 \mathrm{C}} & \text { Compressor inlet temperature } \\ \mathrm{T}_{2 \mathrm{C}} & \text { Compressor discharge temperature } \\ \mathrm{T}_{1 \mathrm{~T}} & \text { Turbine inlet temperature } \\ \mathrm{T}_{2 \mathrm{~T}} & \text { Turbine discharge temperature } \\ \mathrm{PR}_{\mathrm{C}} & \text { Compressor pressure ratio } \\ \mathrm{PR}_{\mathrm{T}} & \text { Turbine pressure ratio } \\ \mathrm{C}_{\mathrm{p}} & \text { Specific heat capacity } \\ \mathrm{N}_{\mathrm{C}} & \text { Physical compressor speed } \\ N_{C}^{*} & \text { Corrected compressor speed } \\ \mathrm{N}_{\mathrm{T}} & \text { Physical turbine speed } \\ N_{T}^{*} & \text { Corrected turbine speed } \\ \mathrm{W}_{\mathrm{C}} & \text { Physical compressor mass flow rate } \\ W_{C}^{*} & \text { Corrected compressor mass flow rate } \\ \mathrm{W}_{\mathrm{T}} & \text { Physical turbine mass flow rate } \\ W_{T}^{*} & \text { Corrected turbine mass flow rate } \\ \dot{W}_{C} & \text { Compressor Power } \\ \dot{W}_{T} & \text { Turbine Power } \\ \mathrm{R} & \text { Gas constant } \\ \bar{R} & \text { Universal gas constant } \\ \mathrm{M} & \text { Molecular weight } \\ \mathrm{h} & \text { Specific enthalpy }\end{array}$

Greek:

$\gamma \quad$ Ratio of specific heats

Acronyms:

ACM Air-cycle machine

BSFC Brake specific fuel consumption

CFM Cubic feet per minute

DAR Dry air rated

SCFM Standard cubic feet per minute

LFE Laminar flow element

CHRA Center housing rotating assembly

$\mathrm{Cl}$ Compression ignition 
SI Spark ignition

COP Coefficient of performance

VE Volumetric efficiency

EGT Exhaust gas temperature 


\section{List of Tables}

Table 2.1 Ambient conditions for theoretical performance model.

Table 2.2 Tabulated data from parametric study of performance analysis for a relative humidity of $50 \%$.

Table 4.1 Operating Conditions for single point comparison of theoretical and actual performance. 38

Table 4.2 Results of single point comparison of theoretical and actual performance. .38

Table 4.3 ACM component weight breakdown.

Table A.1 Measurement Uncertainty Based on Calibration Instrumentation. 47

Table A.2 Values used in calculating uncertainty in ACM compressor efficiency calculations. .... 49

Table A.3 Conditions for evaluating uncertainty in mass flow rate... 49

Table A.4 Conditions for evaluating uncertainty in ACM compressor power. 50 


\section{List of Figures}

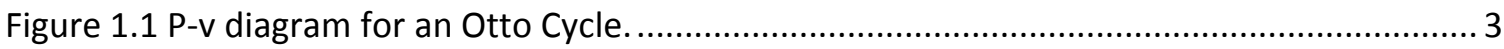

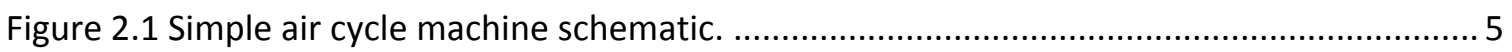

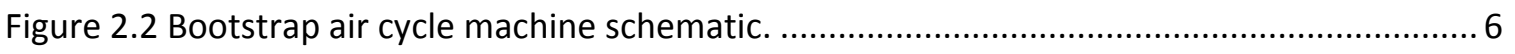

Figure 2.3 On-engine two-wheel bootstrap schematic. ............................................................. 9

Figure 2.4 Comparison of the Reverse-Brayton Air Cooling Cycle and the Brayton Cycle. ........... 14

Figure 2.5 Parametric study of ACM performance for a compressor with $60 \%$ efficiency and varying levels of turbine efficiency and heat exchanger effectiveness........................................ 16

Figure 2.6 Parametric study of ACM performance for a compressor with $80 \%$ efficiency and varying levels of turbine efficiency and heat exchanger effectiveness....................................... 16

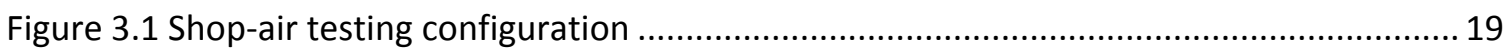

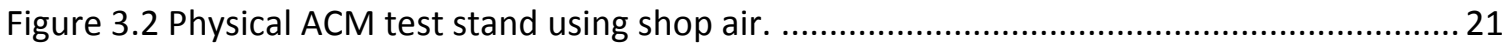

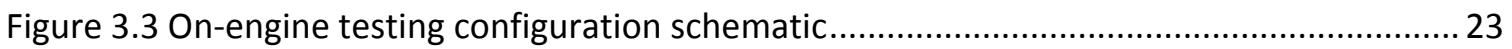

Figure 3.4 Primary engine compressor matching process with an engine operating point of

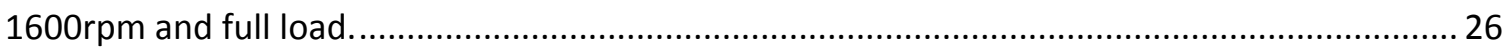

Figure 3.5 Primary engine compressor match with the Perkins diesel boost curve and the target boost curve with the ACM operating.

Figure 4.1 Dry air rated COP for the unmodified GT1241 turbocharger. Data points in

Figures 4.1-4.3 have a one-to-one correspondence.

Figure 4.2 Dry air rated ACM cooling capacity. Data points Figures 4.1-4.3 have a one-to-one correspondence.

Figure 4.3 Intercooler effectiveness variation with mass flow rate in the GT1241 ACM with the electric fan. Data points Figures 4.1-4.3 have a one-to-one correspondence........................30 Figure 4.4 Dry air rated COP for the modified GT1244 turbocharger. Data points Figures 4.4-4.5 have a one-to-one correspondence. 32

Figure 4.5 Dry air rated ACM cooling capacity. Data points Figures 4.4-4.5 have a one-to-one correspondence. 32

Figure 4.6 Intercooler effectiveness variation with ACM mass flow rate using the electric fan.

Data points Figures 4.4-4.5 have a one-to-one correspondence. 33

Figure 4.7 Dry air rated COP for the modified GT1244 turbocharger. Data points

Figures 4.7-4.9 have a one-to-one correspondence. 34

Figure 4.8 Dry air rated ACM cooling capacity. Data points Figures 4.7-4.9 have a

one-to-one correspondence. 35

Figure 4.9 Intercooler effectiveness variation with ACM mass flow rate. Data points

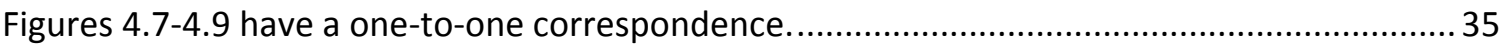

Figure 4.11 P-v diagram for four-stroke gasoline engine. ...................................................... 41

Figure A.1 Uncertainty in ACM compressor efficiency during typical operating conditions......... 48

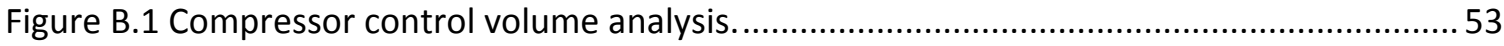

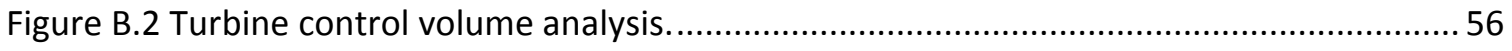

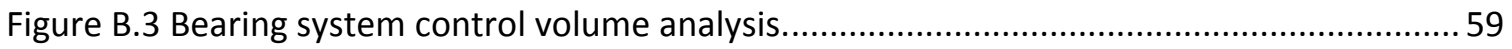




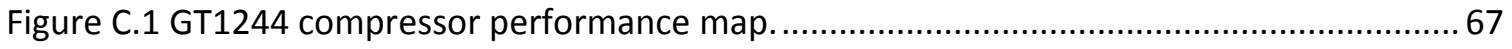

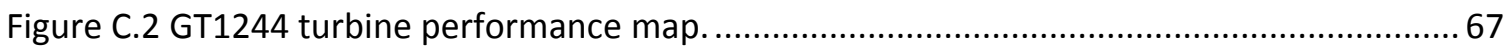

Figure D.1 Thermocouple measurement noise (pre-filtered) before and after shielding

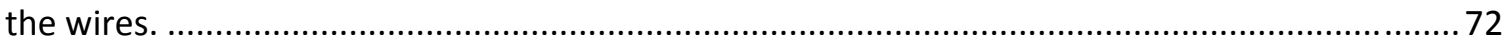

Figure D.2 Temperature measurement output after oversampling and averaging. .................... 72

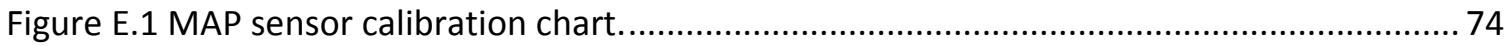

Figure E.2 This schematic shows the configuration of the LFE, differential pressure

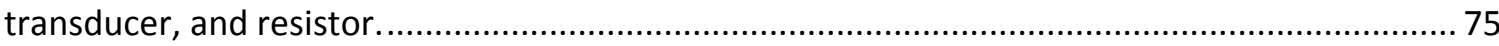

Figure E.3 Laminar flow element calibration curve. Data is provided by Meriam Process

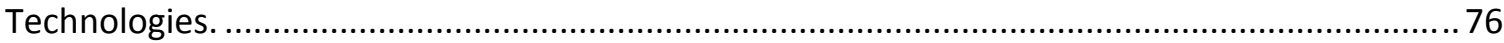

Figure E.4 Differential pressure transducer and resistor combined calibration. ........................... 77

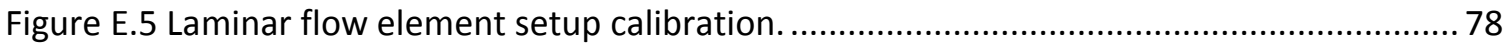

Figure F.1 BSFC contour map using the stock turbocharger.................................................. 79

Figure F.2 VE contour map using the stock turbocharger. ......................................................... 79

Figure F.3 Intercooler effectiveness contour map using the stock turbocharger. ....................... 80

Figure F.4 Pressure differential between the exhaust and intake manifolds. (P_int - P_exh) ...... 80

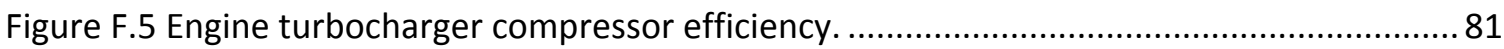

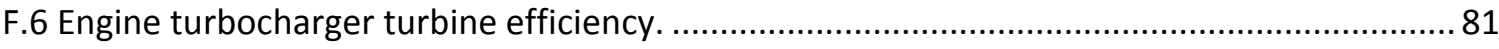

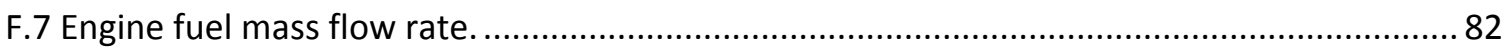

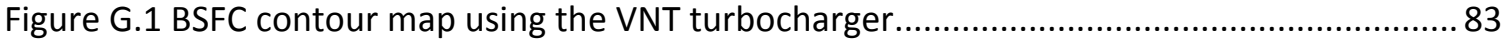

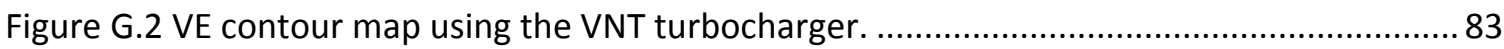

Figure G.3 Intercooler effectiveness contour map using the VNT turbocharger. ........................... 84

Figure G.4 differential between the exhaust and intake manifolds. ( $P_{-}$int - $P_{-}$exh) .................... 84

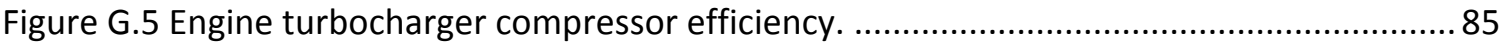

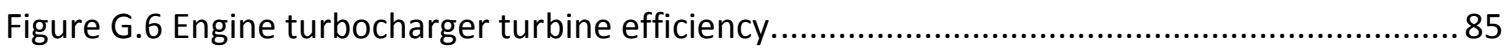

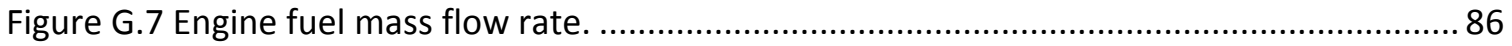




\section{Chapter 1: Introduction}

Automotive air conditioning systems are in the process of undergoing some major changes due to environmental concerns. The new changes are to prevent global warming, in addition to ozone depletion. These changes tend to have negative impacts on air conditioning performance due to a shift in refrigerant selection criteria, from maximum coefficient of performance (COP) to lifecycle environmental impact of the system. The new criteria considers designs that have lower COP but may still have less overall negative impact on the environment throughout the lifecycle of the system. Some of the other considerations in selecting a refrigerant, are the system weight, manufacturing costs, refrigerant toxicity, and effects of potential leaks. Looking into the future, additional changes to automotive air conditioning systems are likely, and use of a refrigerant that is naturally available and does not need to be retrofitted is convenient.

Air-cycle machines (ACM) conveniently use air as the refrigerant and have the potential to provide convenient air conditioning for high performance vehicles, where weight is at a premium. A typical ACM can consist of a compressor, heat exchanger, and cooling turbine, which is supplied with pressurized air. The main components of an air-cycle cooling system are the compression process, heat removal, and controlled expansion. The cool air from the turbine outlet can be routed directly into the passenger cabin.

In racing applications air conditioning is a secondary consideration in vehicle design, and often not used due to weight and power requirements. Any amount of cooling that can be provided without loss in engine performance and minimal weight increase is considered to be an improvement. A bleed-air control configuration is best-suited to racing applications because the amount of cooling required is not set, any cooling provided is beneficial. The bleed-air 
configuration requires a forced-induction system as the primary source of compressed air for the Reverse Brayton Cycle to operate. This configuration will work with either a supercharged or turbocharged system. The latter is preferred to minimize the parasitic power loss on the engine caused by the ACM operation.

Instead of "waste-gating", a process in which a fraction of the exhaust to bypass the turbine stage to limit compressor outlet pressure, the compressor outlet pressure can be limited by bleeding air through the ACM. This has a reduced impact on power consumption from the engine because the power would have gone to waste if not used for cooling.

A variable nozzle turbine (VNT) can be used to achieve a desired cooling capacity while maintaining boost levels with increased primary compressor power requirements during full $\mathrm{ACM}$ operation by varying turbine power. This configuration can be designed to increase overall system efficiency by minimizing lower-loop losses, which is important for minimizing fuel consumption. The lower-loop losses can be seen on the P-v diagram of the Otto Cycle, in Figure 1.1. The lower-loop on either the Otto or Diesel Cycles represents the net pumping losses induced on the engine by the exhaust and intake systems. The net power can be determined by integrating the P-v curve forward through the intake and exhaust strokes. The sum of the signed areas results in the net power consumed. An intake pressure greater than the exhaust backpressure will result in a negative pumping loss, or increased engine power. 


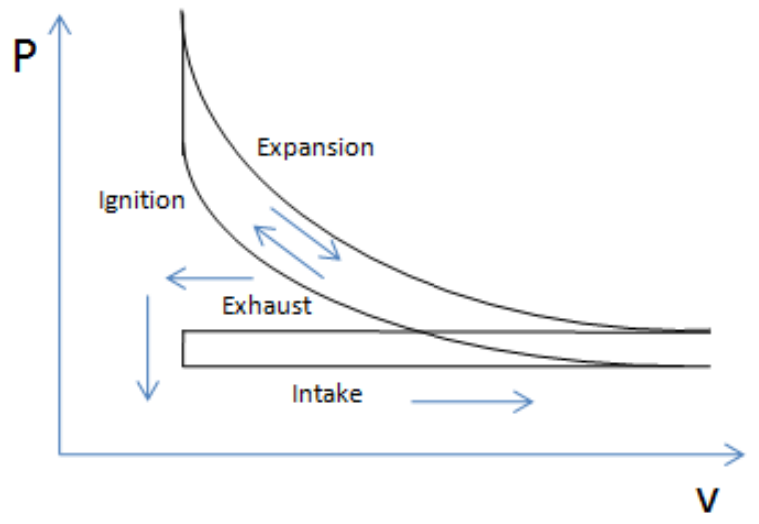

Figure 1.1 P-v diagram for an Otto Cycle.

The VNT control method was found to be the most practical to implement for this testing, and it demonstrates that the primary turbocharger can supply the necessary airflow to operate the ACM effectively in a bleed-air configuration. There are two objectives in this project; 1 )

demonstrate the feasibility of using commercially available automotive turbocharger components to design an ACM unit; and, 2) demonstrate that an engine-turbocharger system can be re-designed to support the additional airflow required by the ACM. 


\section{Chapter 2: Background}

Air-cycle technology has been in use since the 1940's in aircraft environmental control systems (ECS) [1]. Until then it was considered commercially unfeasible due to its relatively low coefficient of performance (COP) compared to other refrigeration methods available, namely vapor-cycle systems and dry ice. The appeal that made it a consideration for use in aircraft is the size and weight of a typical ACM. In addition to this, there is a convenient source of compressed air on-board already, the jet engine's compressor stages. For comparable systems, in terms of cooling capacity, air-cycle systems can reduce space requirements by approximately $25 \%$ and weight by $50 \%$ [1]. This is important in racing and high-performance applications because engine bays are typically very tight on space, and weight can diminish acceleration and handling. The increased weight can be especially detrimental in vehicles with down-force since the increase in weight does not necessarily increase the lateral load capability by a proportional amount.

The air-cycle machine is based around the concept of a cooling turbine. This is the common component among all of the various ACM configurations, such as the simple, two-wheel, or three-wheel bootstrap cycles. The cooling turbine operates by providing a controlled expansion of air; an adiabatic, controlled expansion requires the air to do work on its boundaries to provide any cooling effect. The work done on the turbine wheel is transmitted by the shaft to a compressor or fan to utilize this power. The compressor and fan are methods of dissipating turbine power to do useful work in the system. 


\subsection{Simple Cycle}

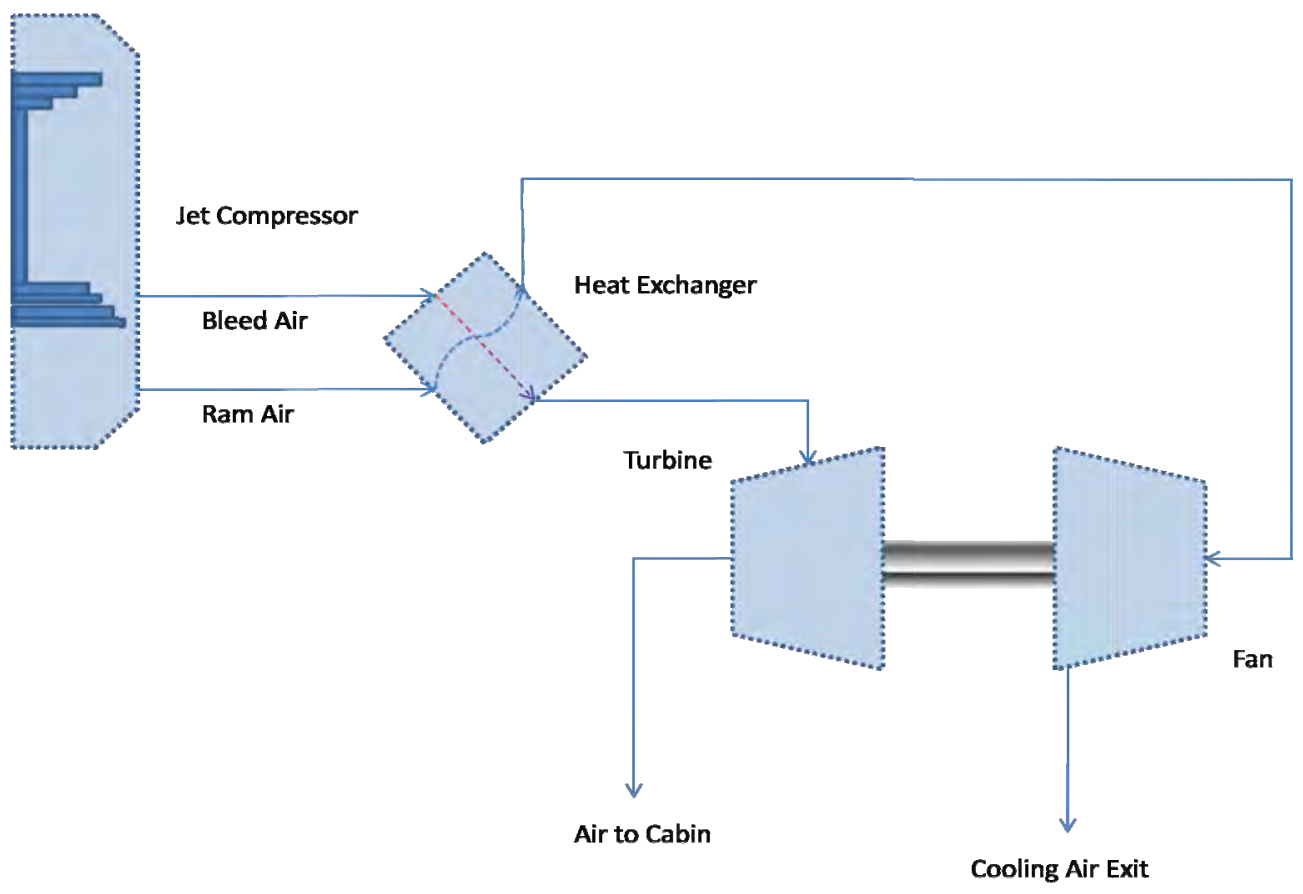

Figure 2.1 Simple air cycle machine schematic.

The main advantage to using the simple air-cycle system, as the name implies, is simplicity.

Decreased component numbers reduce weight and space requirements. The air coming from the main jet engine is typically in the range of $400-600^{\circ} \mathrm{F}$. This air is then passed through a heat exchanger with high effectiveness to get the air cooled down to as close to the ram air temperature as possible. Once the air is cooled by the heat exchanger, it is introduced into the turbine for expansion. The power produced by the turbine is transmitted, by direct drive, to the fan. The turbine power corresponds to the enthalpy change in the air passing through it. There are two components to cooling the air; 1) Heat rejection from the ACM intercooler; 2) Turbine power derived from the airflow reduces enthalpy and air temperature. The fan helps improve heat exchanger effectiveness by increasing the airflow over the cold side of the heat exchanger. The fact that the simple cycle utilizes a fan and does not rely only on ram air for cooling the heat 
exchanger allows it to provide cooling while the plane is stationary. This is not ideal for applications with the intent of using typical automotive turbocharger components because of fabrication required for manufacturing and installing the fan on the turbine shaft.

\subsection{Two-wheel Bootstrap Cycle}

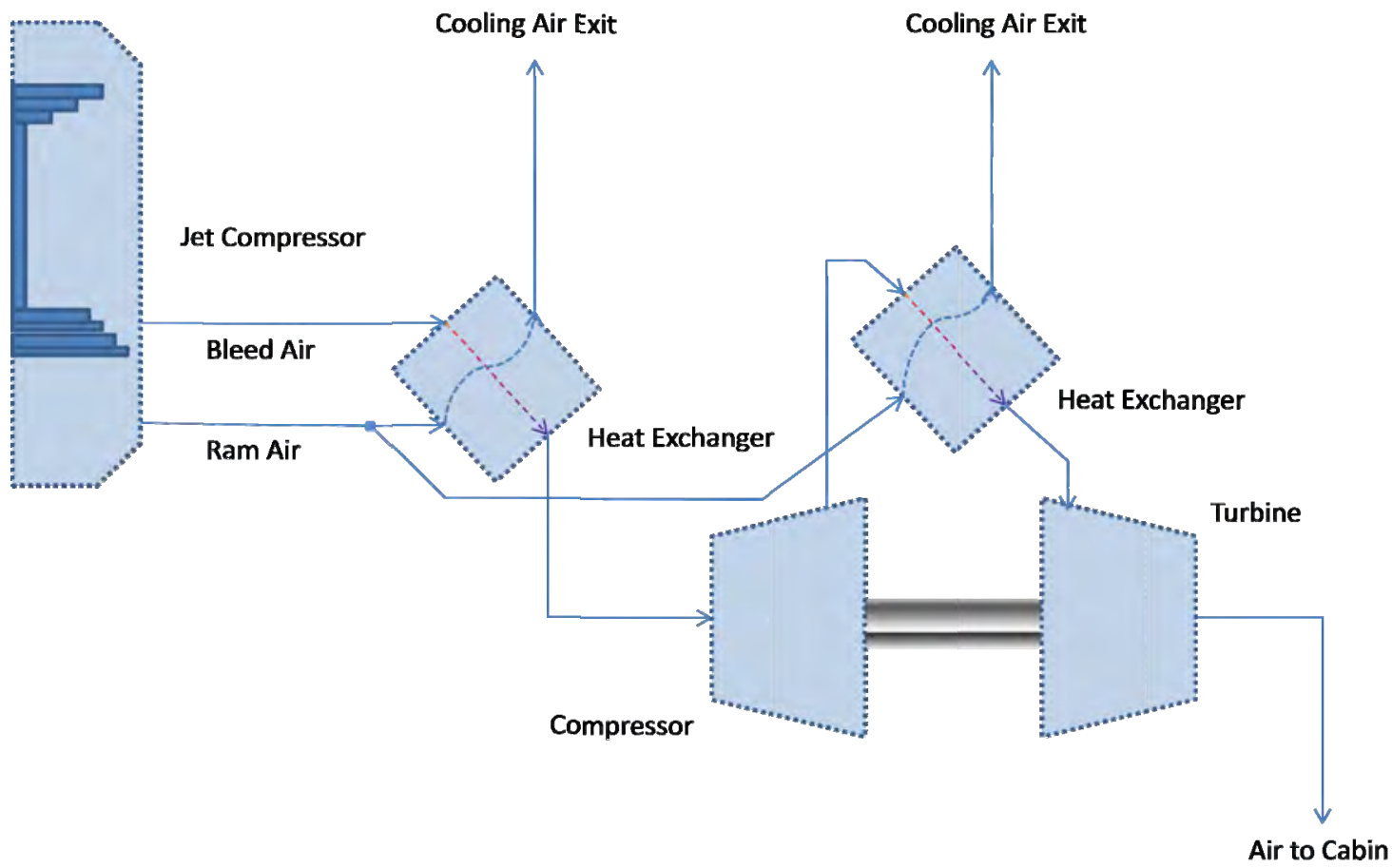

Figure 2.2 Bootstrap air cycle machine schematic.

The most noticeable differences from the simple air cycle machine are the use of a compressor, instead of a fan, and a second heat exchanger. While this system relies on ram air for cooling and does not provide cooling while the aircraft is stationary, it does provide significantly lower turbine discharge temperatures. The turbine discharge temperature is low enough to have concerns about freezing the moisture out of the airflow. Although the ice does not form on the turbine wheel itself, it can build up to an amount that restricts airflow to the cabin, reducing the pressure ratio across the turbine and decreasing performance. 
The air is drawn from one of the main engine compressor stages and cooled through a primary heat exchanger, just as the simple cycle does. Next, the cooler air enters the secondary compressor stage. This provides another pressure rise to further compress and heat the air. The power required by the secondary compressor to accomplish this task is provided by the cooling turbine. The air exiting the secondary compressor stage is cooled by another heat exchanger before entering the turbine. The turbine in the bootstrap configuration receives air at approximately the same temperature as the simple cycle, but at a significantly higher pressure ratio across the turbine. This is how the bootstrap air cycle machine is able to provide lower turbine discharge temperatures than the simple cycle.

\subsection{Three-wheel Bootstrap Cycle}

The three-wheeled bootstrap cycle is essentially the same as the two-wheeled assembly with the addition of a fan mounted on the same shaft as the ACM compressor and turbine or geardriven from the shaft. The fan ensures adequate airflow on the cold side of the intercooler, but it will consume power from the turbine and reduce the pressure ratio across the compressor. This configuration requires one-off design and fabrication of ACM components that are not readily available with automotive parts.

\subsection{Project Goals}

The goal of this project is to demonstrate that an ACM air conditioning unit can be manufactured from existing automotive turbocharger components and provide acceptable cooling with a minimal performance penalty. There are many choices for ACM configuration, but some of them lend themselves more easily to automotive applications than others.

The simple cycle has few components, but the turbocharger compressor would have to be replaced by a fan. This requires a significant amount of fabrication and does not meet the goal 
of using existing automotive turbocharger components. Using a fan mounted on the turbine shaft requires ducting that may be difficult to package in a car engine bay.

The three-wheel bootstrap configuration makes for a nicely-packaged rotating assembly. The main concern with this setup is the manufacturing and balancing of a complicated rotating assembly. This requires extensive modification to a turbocharger and is not appropriate for meeting the goals of this project.

The two-wheel bootstrap configuration requires more components, but there is a compressorturbine assembly that allows the use of a turbocharger to package those components. An electric fan is needed to provide airflow over the intercooler at low vehicle speeds. This allows for simpler packaging in a small engine bay than ducting air from a remotely located fan. The two-wheel configuration provides the lowest turbine discharge temperature of all the setups. This is important in an automotive application because the pressure ratios available are limited due to engine constraints. A lower pressure ratio will reduce the temperature drop across the turbine, assuming everything else remains constant. The two-wheel bootstrap configuration is used for this project because it meets the goals that have been set. This configuration, in the context of the engine-mounted system to be tested in this project, can be seen below. 


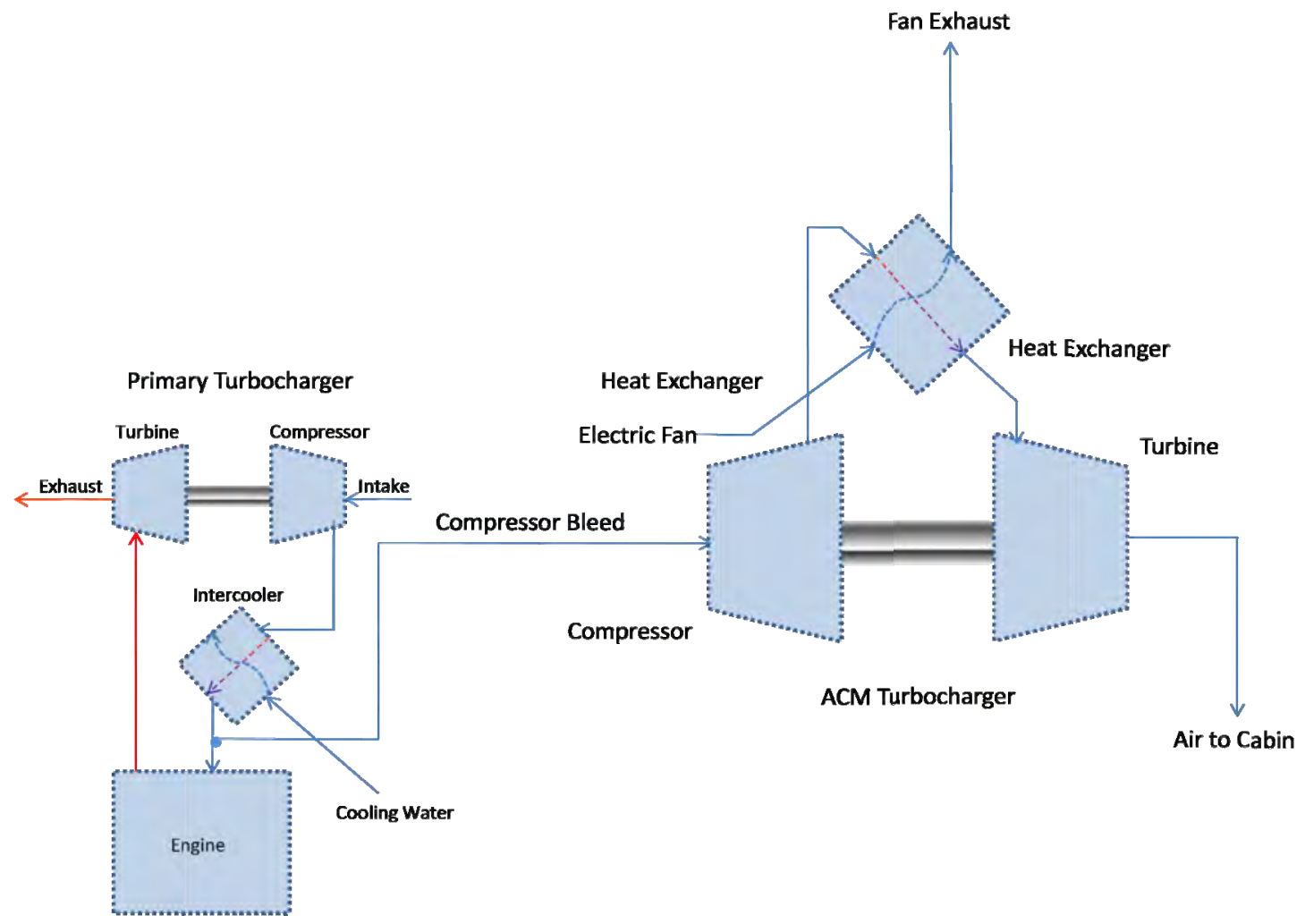

Figure 2.3 On-engine two-wheel bootstrap schematic.

The on-engine ACM configuration operates by bleeding air from the engine's forced-induction pressurized intake tubing to the intake manifold. The bleed-air configuration allows for control of intake manifold pressure or boost, a common name referring to intake manifold gauge pressure. Boost control is typically performed by using a pneumatic actuator that senses compressor outlet pressure and closes or opens a flapper valve located next to the turbine. This allows for a fraction of the exhaust gas to bypass the turbine, limiting turbine power and boost. The turbine bypass valve is commonly referred to as a waste-gate. It is referred to as "wastegating" because any fraction of the exhaust that is allowed to bypass the turbine is wasted energy. The compressor bleed configuration uses this wasted energy to power the ACM while preventing excessive boost. 
While the on-engine, two-wheel bootstrap configuration is the primary goal of this project, the turbine-compressor match must first be validated. This is performed on a stand-alone test stand with compressed air supplied to the ACM by the shop air compressor system. This configuration can be seen in Figure 3.1.

\subsection{COP Defined}

Traditionally COP is defined as the ratio of cooling load compared to the power required to drive the system. This is a practical definition for most air conditioning systems where the compressor, dynamic or fixed-displacement, is driven from a motor or engine. The power, with an associated cost, going to the air conditioning system is easier to determine than that of the turbocharger-based system described in this project since there is a physical connection between the motor and compressor.

$$
\text { COP traditional }=\frac{\dot{Q}_{\text {cooling }}}{\dot{W}_{\text {compressor,fans }}}(\text { Eqn. 2.1) }
$$

This is not as clear when considering an ACM powered by the engine's turbocharger; not all of the power delivered to the ACM has a cost associated with it. A portion of the power that went to drive the primary compressor was from heat energy that would have been wasted through the exhaust anyways or lost through waste-gating.

A new method for determining power consumption specifically by the ACM is proposed. This can be done by finding the change in fuel flow rate with and without the ACM operating and using brake-specific fuel consumption (bsfc) to calculate power used by the ACM. This represents the effective power to drive the ACM, in other words, only power that has an associated increase in fuel cost. This applies to ACM operation outside of normal waste-gate 
operation to limit boost. ACM operation to limit boost levels instead of using the waste-gate has minimal impact on engine fuel consumption, lending to a significantly increased COP.

$$
\begin{aligned}
& C O P_{\text {effective }}=\frac{\dot{Q}_{\text {cooling }}}{\dot{W}_{\text {Engine-ACM }}}(\text { Eqn. 2.2) } \\
& \dot{W}_{\text {Engine-ACM }}=\frac{\Delta \dot{m}_{\text {fuel,ACM }}}{b s f c} \quad(\text { Eqn. 2.3) }
\end{aligned}
$$

$\mathrm{COP}_{\text {effective }}$ is not the only concern in $\mathrm{ACM}$ design; the cooling capacity is the driving requirement in the design process. In optimizing the system, while meeting cooling capacity requirements, COP is maximized for a given set of ambient and ACM inlet conditions. The effective COP and cooling capacity together are referred to as ACM performance.

\subsection{Previous work performed at Queen's University, Belfast}

To the author's knowledge, there has only been one other recent group to perform air-cycle research for automotive applications. The idea of using air-cycle air conditioning for this type of application is well-founded by theory, but practical limitations in implementing the system can considerably decrease the performance of an ACM. This can be observed in the previous work described below.

A research group at Queen's University, Belfast, has designed and implemented a superchargerbased ACM for refrigerated trailers in road transport applications $[2,3]$. The group implemented a two-wheel bootstrap cycle similar to the one in this project. The primary compressor is geardriven from the crankshaft, and it feeds compressed air to the typical bootstrap ACM. Instead of bleeding air from the engine intake manifold as done in this project, the supercharger is dedicated to supplying air to the ACM. This configuration somewhat decouples engine and ACM operation, making it easier to control and quantify ACM performance. It has a severe 
shortcoming in that it will always be a parasitic power loss from the engine crankshaft, rather than "free" power from a turbocharger-based system that is demonstrated in this system. The theoretical COP of their ACM was 0.294 , corresponding to the $\mathrm{COP}_{\text {traditional }}$ defined above, while an optimized model showed a possibility of 0.62 . Their performance goal was not achieved due to excessively low efficiency of the gearbox to drive the supercharger, heat exchanger performance, and excessively large turbocharger bearing losses. Overall, the fuel consumption of the engine was approximately three times greater with the ACM operating, and no actual COP figures were provided from this testing. While their testing did not show promising results, the lower-than-expected performance likely came from limitations in components selected rather than the air-cycle concept itself.

The turbocharger-based ACM differs from the supercharger-based unit because there is energy available that would have been wasted during the turbine bypass process, waste-gating, without the presence of the ACM. Instead of wasting this energy, it can be utilized for powering the ACM without any additional cost to the engine. This is essentially free cooling, or infinite COP. Since the use of a turbocharger as the air source allows increased upper limits of performance than the supercharger-based system, there is an improved likelihood of success.

\subsection{Theoretical ACM Performance}

Air-cycle analysis can be performed assuming dry or moist air. The analysis for this project assumes dry air, except for using the dry-air rated (DAR) analysis for cooling capacity. DAR temperature is the equivalent temperature if the entrained water or ice in the air exiting the turbine is adiabatically evaporated [4]. Dry air is assumed because, for most of the system, the difference in enthalpy change across components is minimal between the two methods. This, however, is not true for the turbine because the air temperature falls below that of the ambient 
conditions and moisture can drop out as a liquid, or possibly ice. This can have a significant impact in the enthalpy change across the turbine, affecting turbine power and discharge temperature. Dry air is assumed for simplicity in designing the first prototype. Detailed derivations of the dry-air, perfect gas equations can be found in Appendix B.

The turbine-compressor matching process for an ACM is different than that of a match performed for an engine application. This is primarily due to the difference in turbine inlet conditions. Corrected parameters, such as flow and speed, for the turbine are dependent upon temperature. The turbine inlet temperature is significantly lower for an ACM than typical exhaust gas temperatures of either diesel or gasoline engines. This presents a challenge because commercial turbochargers are designed for engine applications, and it requires a new compressor to be matched to the turbine to maximize performance in the ACM application. The compressor-turbine matching process can be found in Appendix C. The differences in the typical on-engine application and the ACM application become apparent when viewing a T-s diagram with both the Brayton and Reverse-Brayton cycles. The Brayton Cycle is modeling the engine as a heat input to the turbocharger system. Figure 2.4 shows the relative temperature and entropy changes through each cycle.

T he process starts at the compressor inlet and the volume decreases as the pressure increases. The Brayton Air-Standard Cycle is modeled with the engine as a continuous thermodynamic machine and with a mass-fraction bypassing the turbine to prevent over-pressurizing the intake. The intake air is then drawn into the engine where it goes through the combustion process where the burned fuel adds heat to the flow before it is discharged into the exhaust manifold. The exhaust flow increase volume and decreases in pressure as it expands through the turbine. A line connecting the turbine outlet and the compressor outlet represents the air possibly being 
recycled at some point and closes the cycle. The Reverse-Brayton Cycle involves much less energy than compared to the Brayton Cycle. This results in changes in corrected conditions for the compressor and turbine, lower compressor pressure ratios, and requires a somewhat different approach to the compressor-turbine matching process. A relatively larger compressor wheel proves to be a better match for reducing excessive corrected turbine speeds for maximizing turbine efficiency, while still keeping compressor efficiency in mind.

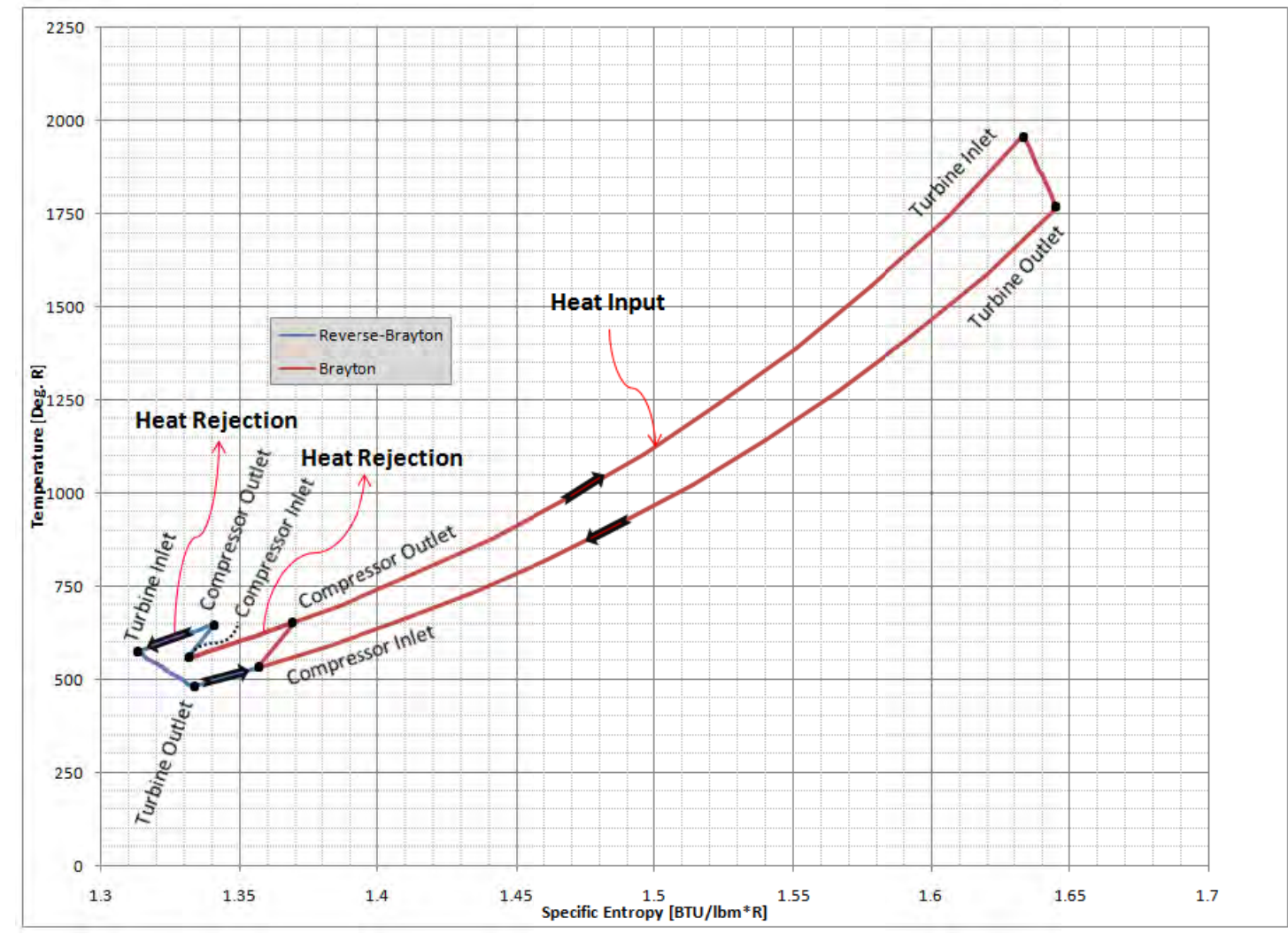

Figure 2.4 Comparison of the Reverse-Brayton Air Cooling Cycle and the Brayton Cycle.

There are a few things known about the system, as with many systems, such as mass balance, energy balance, and operating speeds of the compressor and turbine wheels. Assuming there are no leaks in the system, the mass flow rate through the compressor and turbine will be the same. With the assumption that the bearing losses are negligible, which is the case in most 
engine applications where the uncertainty of many other parameters outweigh this, the power output of the turbine will be matched by the compressor power requirement in steady-state operation. Considering the compressor and turbine wheels are directly mounted on the same shaft, the shaft speeds will be equal. Even with as many known parameters as there are, it is an iterative process to find a mass, power, and speed balance because of the graphical nature of the compressor and turbine performance maps.

It is important to investigate the effects of various component performances on overall ACM performance. A parametric study of ACM component efficiencies was conducted and the results are in Figure 2.5-2.6 and Table 2.2. With the results from the parametric study, attention can be given to the most critical components first. The analysis presented next shows that heat exchanger effectiveness (E_htxr2, Figure 2.5-2.6) and turbine efficiency (Eta_t2, Figure 2.5-2.6) are more important to overall system performance than compressor efficiency. The data in the following figures are calculated assuming a primary compressor efficiency of $70 \%$ and ambient conditions similar to those found at the testing location. The compressor efficiency chosen is representative of attainable performance over a wide range in air flow in modern automotive turbocharger systems [6]. The code used to generate the following plots can be found in Appendix K.

Table 2.1 Ambient conditions for theoretical performance model.

\begin{tabular}{ll} 
Ambient Condition & \\
\hline Temperature [Deg. F] & 70 \\
Pressure [psia] & 14.69 \\
Relative Humidity [-] & 0.50
\end{tabular}




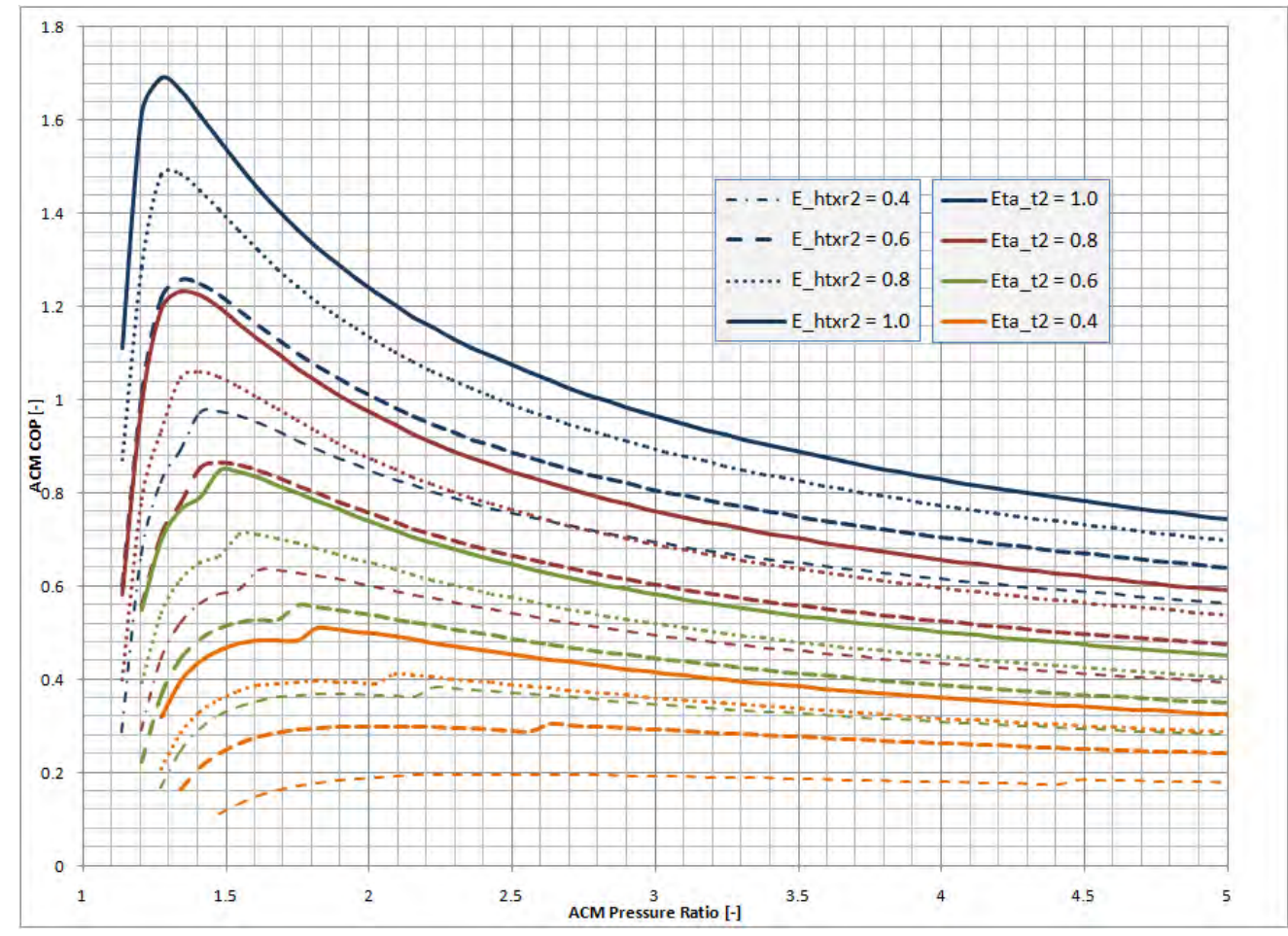

Figure 2.5 Parametric study of ACM performance for a compressor with $60 \%$ efficiency and varying levels of turbine efficiency and heat exchanger effectiveness.

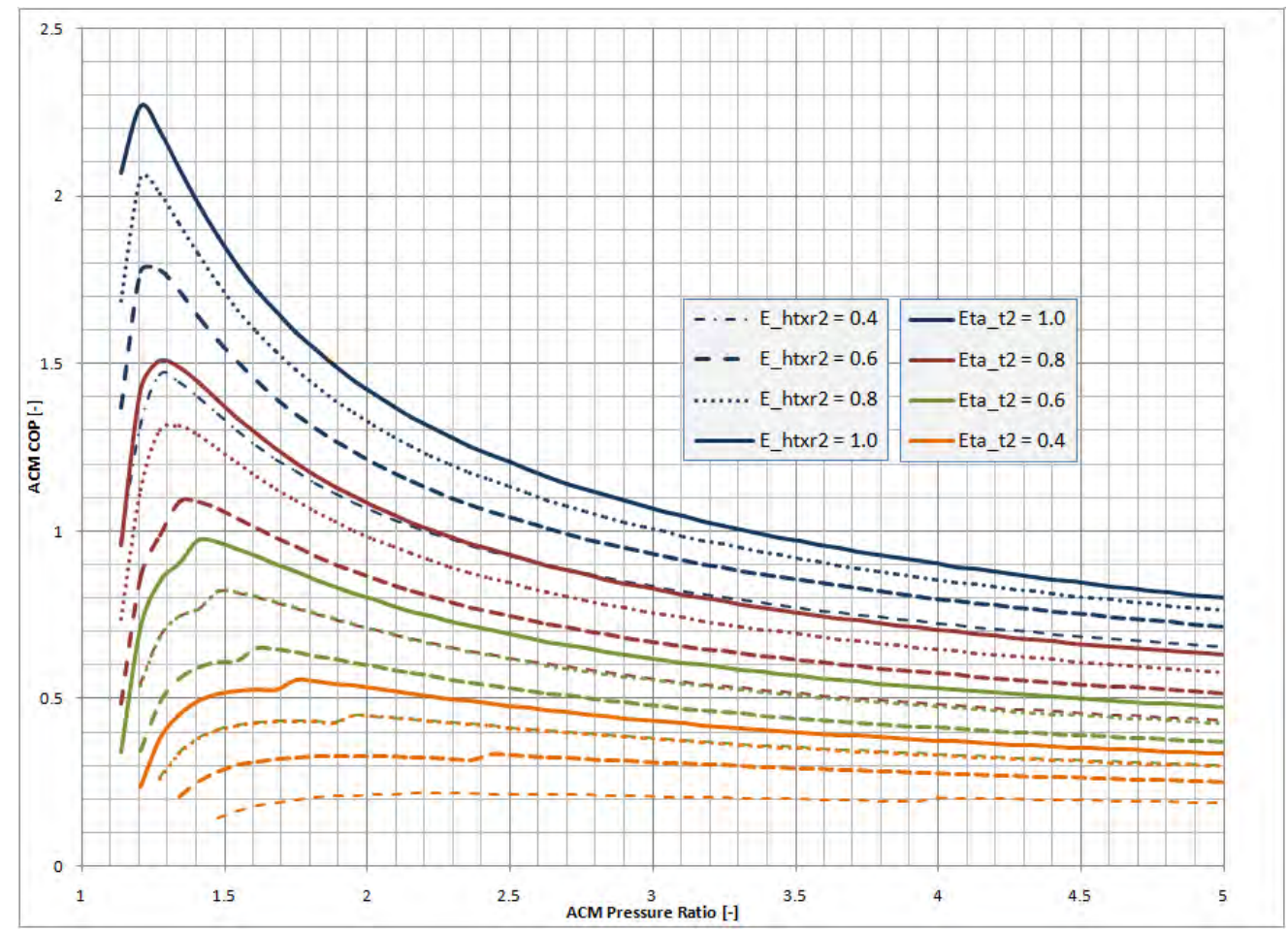

Figure 2.6 Parametric study of ACM performance for a compressor with $80 \%$ efficiency and varying levels of turbine efficiency and heat exchanger effectiveness. 
This analysis indicates that minimum acceptable intercooler effectiveness is approximately 0.70 before rapidly decreasing overall ACM performance. While compressor and turbine efficiencies remain constant, the curves for ACM COP decrease by larger amounts as heat exchanger effectiveness decreases.

Using representative values from the previous analysis, attainable performance with automotive turbocharger components, for intercooler effectiveness, turbine efficiency, and compressor efficiency, the following table was constructed:

Table 2.2 Tabulated data from parametric study of performance analysis for a relative humidity of $50 \%$.

\begin{tabular}{|l|l|l|l|l|}
\hline $\begin{array}{c}\text { Pressure Ratio } \\
{[-]}\end{array}$ & \multicolumn{1}{|c|}{$\begin{array}{c}\text { Intercooler } \\
\text { Effectiveness [-] }\end{array}$} & \multicolumn{1}{|c|}{$\begin{array}{c}\text { Turbine } \\
\text { Efficiency [-] }\end{array}$} & $\begin{array}{c}\text { Compressor } \\
\text { Efficiency [-] }\end{array}$ & \multicolumn{1}{|c|}{ COP $_{\text {DAR }}[-]$} \\
\hline 1.5 & 0.80 & 0.80 & $0.60 \rightarrow 0.80$ & $1.05 \rightarrow 1.25$ \\
\hline & & $0.60 \rightarrow 0.80$ & 0.80 & $0.82 \rightarrow 1.25$ \\
\hline & 0.60 & 0.80 & $0.60 \rightarrow 0.80$ & $0.86 \rightarrow 1.07$ \\
\hline & & $0.60 \rightarrow 0.80$ & 0.80 & $0.61 \rightarrow 1.07$ \\
\hline
\end{tabular}

It can be observed that the turbine performance is more critical to the overall cycle performance. This is to be expected because the more efficient the turbine is, the more heat will be removed from the air expanding through the turbine, and the more power will be extracted to drive higher pressure ratios across the compressor. The higher pressure and temperature entering the heat exchanger allows more heat to be removed before entering the turbine. Obviously, there is a limit to this cycle and a steady-state operating point because of the decreasing energy content in the air flow to the turbine as more heat is removed by the intercooler.

It should be noted that the ACM in this testing is operated at the lower pressure ratio range due to boost limitations on the test engine and typical boost pressures for gasoline engines. The theoretical analysis is extended to the upper limits of pressure ratios that can be achieved by 
single stage compression. This is representative of what can be seen in operating intake pressures in diesel engines. 


\section{Chapter 3 Experimental Apparatus, Procedures, and Conditions}

There are two testing configurations needed to complete testing for this project. The first is designed to verify the compressor-turbine match for the ACM itself and to demonstrate that the desired performance can be achieved. The second test is designed to demonstrate on-engine performance.

\subsection{Shop-air Test Configuration}

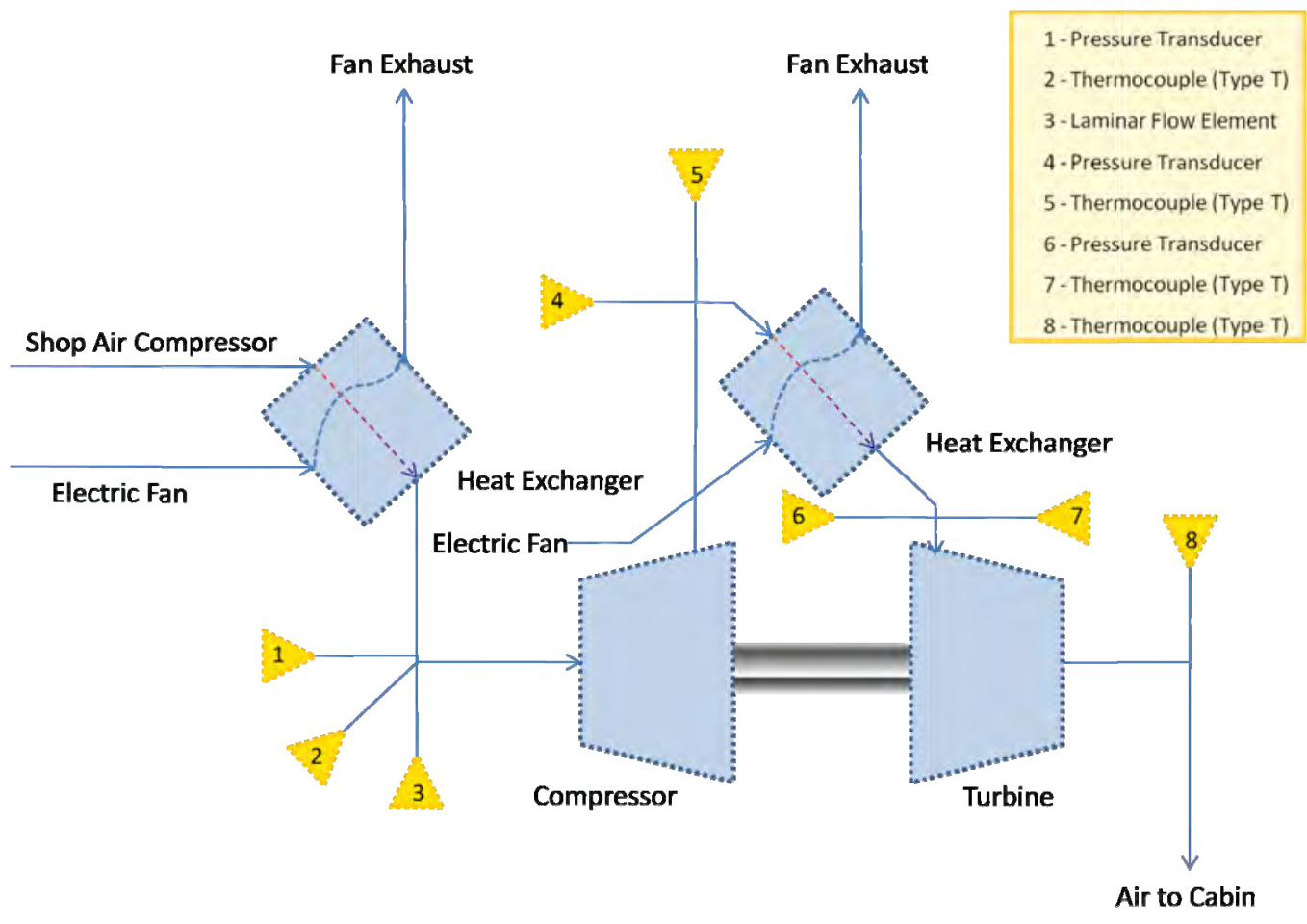

Figure 3.1 Shop-air testing configuration

The first set of tests consists of the ACM turbocharger, intercooler, and required ducting between components powered by shop air. Figure 3.1 shows the connection to the compressed air source and the locations of the sensors. The test facility has a 75-hp reciprocating compressor and large air storage tanks. A relatively large shop air supply is needed because the compressor has to supply the tanks with $125 \mathrm{psig}$, and the ACM air supply is regulated down to a 
nominal 11psig. This pressure represents the ACM inlet conditions that will be similar to operation with the diesel engine. With this in mind, there is not an exact method for finding primary compressor power for COP calculations. To represent realistic primary compressor performance in a typical turbocharger application, an isentropic compressor efficiency of $70 \%$ is assumed. This assumption applies to the following equation to calculate the air source compressor power requirements, solely for the purpose of COP estimation:

$$
\dot{W}_{C}=\dot{m} c_{p} \frac{T_{1}}{\eta_{c}}\left[P R_{C}{ }^{\gamma-1 / \gamma}-1\right] \quad(\text { Eqn. 3.1) }
$$

This testing configuration allows for a baseline to be established with the unmodified, Garrett GT1241, turbocharger. Garret Turbochargers manufactures a large range of turbocharger sizes and many combinations of compressors and turbines. The turbocharger base model selected has the smallest turbine in the product lineup, but it closely matches the requirements for this project. The next step is to optimize performance for an ACM application, rather than a gasoline engine application. This involves sizing another compressor for the GT12 turbine. The compressor-turbine matching process is discussed in greater detail in Appendix C. 


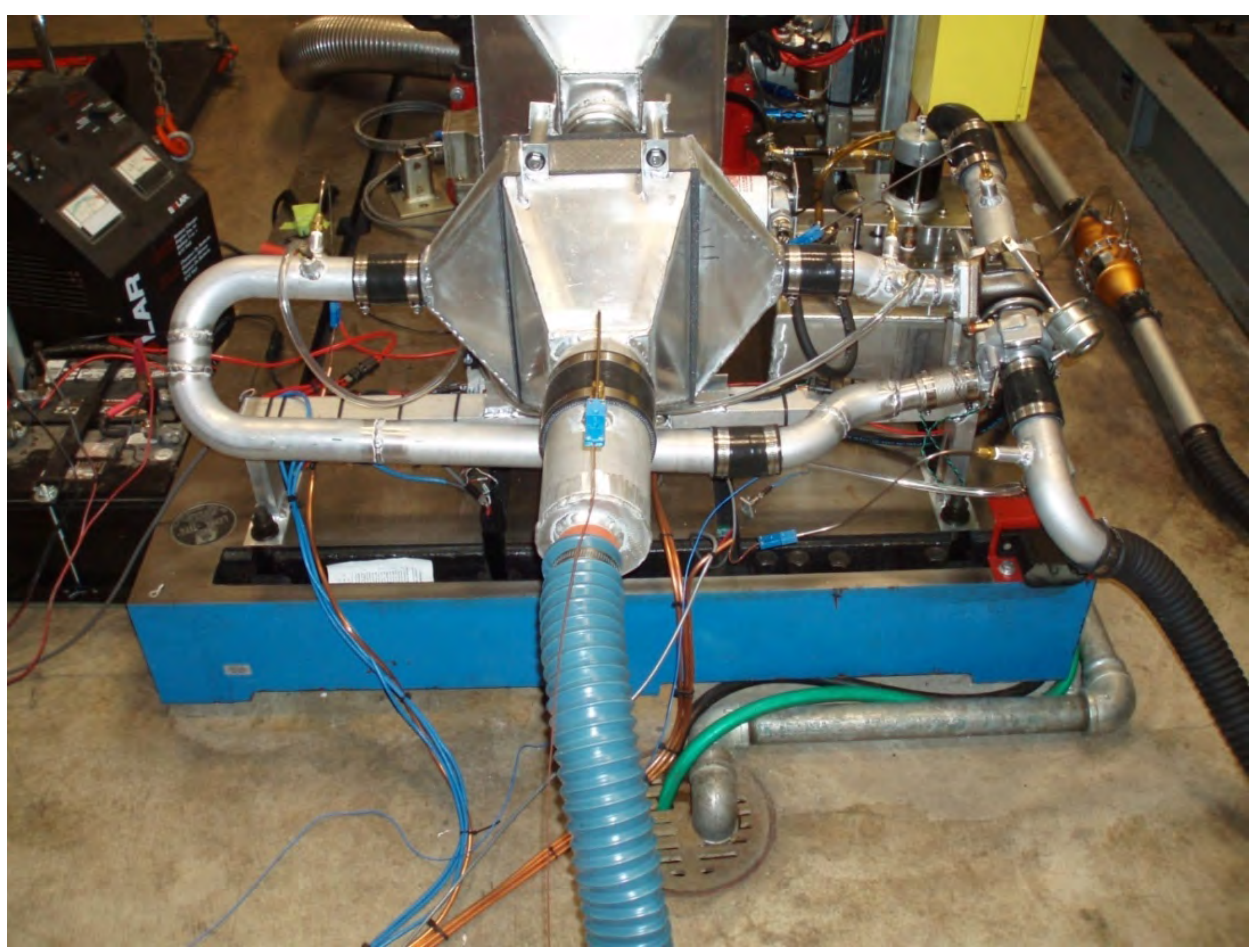

Figure 3.2 Physical ACM test stand using shop air.

Type-T thermocouples were used because they work well with the lower temperature range that the ACM will be operating in. In addition to this, the standard limit of error for type-T thermocouples is $1^{\circ} \mathrm{C}$, compared to $2.2^{\circ} \mathrm{C}$ for type-K. Each thermocouple was calibrated within the standard limit of error. All sensors were checked periodically between sets of experiments to ensure quality data. The calibration data are in Appendix E.

This testing configuration gathered data for a baseline using an unmodified GT12 turbine- $41 \mathrm{~mm}$ compressor wheel assembly for the ACM and a GT12 turbine-44mm compressor wheel assembly for improved efficiency. The larger compressor reduced corrected turbine speeds to improve performance. The $41 \mathrm{~mm}$ compressor wheel placed corrected turbine speed operating point off the high end of the turbine performance map. The compressor provided similar efficiency contours to the smaller compressor wheel, so there wasn't a significant decrease in performance on that end. The differences in the unmodified GT1241 and modified GT1244 
compressor-turbine matches are due to the differences in the component inlet operating conditions for the intended applications, as discussed in Section 2.7.

The installation of the new compressor required manufacturing a shaft adapter due to a larger bore size in the larger compressor wheel. The specifications from Garrett Turbochargers indicated that bore inside-to- outside diameter runout and perpendicularity to the end faces need to be within 0.0001 inch. A shaft adapter was produced that met the acceptable vibration limits at peak operating speeds. This was verified before continuing with testing by slowly increasing the turbocharger speed, while directly measuring shaft speed and bearing housing vibration levels.

The procedure for performing the tests on both the unmodified GT1241 and modified GT1244 assemblies included: blowing out liquid water from the shop air lines and tanks, gathering local ambient conditions, and manually regulating the ACM inlet pressure to achieve desired operating points and steady-state conditions before collecting each data set. Steady-state operating conditions can be detected and verified several ways, such as monitoring shaft speed, temperatures, and pressures.

The component that contributed most to the length of time required to reach steady state was the intercooler. It has a relatively large mass and took some time to reach a steady temperature. It is very important to wait for steady-state conditions before collecting data because fictitiously high performance numbers can be observed. This is because of the thermal capacitance of the aluminum comprising the intercooler core and end caps; it provides a second means of heat removal from the airflow until the intercooler is saturated with heat. Once the intercooler is "heat-soaked", the only means of dissipating heat is to the cold air flow stream, instead of transferring heat from the hot air stream to the thermal-capacitance of the aluminum 
and to the cold air flow stream. Initial testing showed large apparent intercooler effectiveness during the transient period. All data was collected at steady-state conditions for this project, eliminating this effect.

The shop air stand testing demonstrated that the baseline GT1241 turbocharger provided an adequate compressor-turbine match, while the modified GT1244 turbocharger provided a larger useable ACM operating range by increasing the efficiency over that range closer to the peak efficiency. The results of the shop air test stand experiments are discussed in Section 4.1 and Section 4.2. The uncertainty calculations for these measurements can be found in Appendix A. With the compressor-turbine match verified and the performance of the ACM optimized, the next step is on-engine testing.

\subsection{On-engine Testing Configuration}

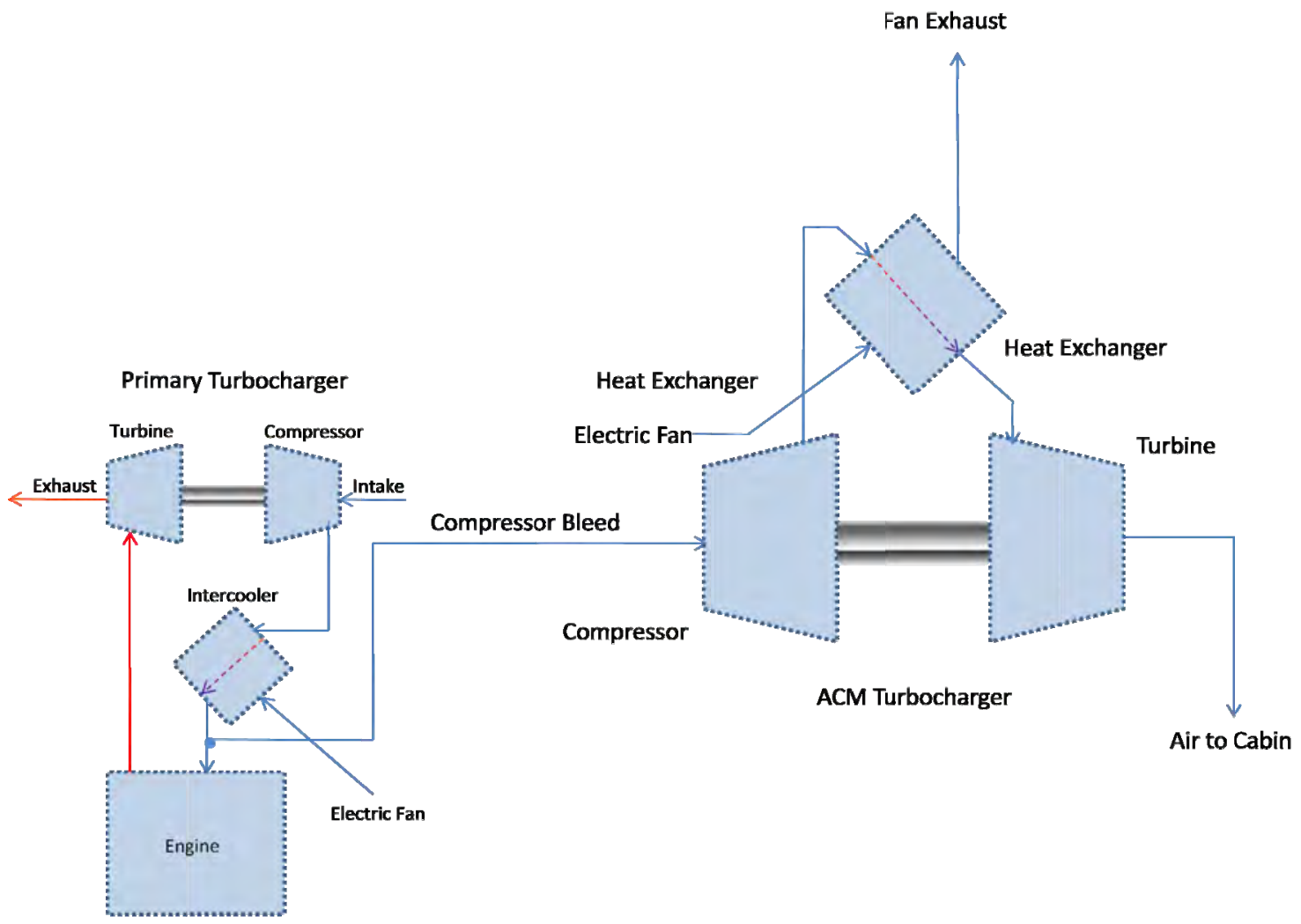

Figure 3.3 On-engine testing configuration schematic 
The on-engine configuration uses the same ACM test stand configuration with the exception of the compressor inlet being fed compressed air from the intake manifold of the diesel engine. This was achieved using a y-pipe after the primary, or engine, intercooler. The flow control valve is located before the ACM compressor inlet and is capable of turning the flow to the ACM on or off and throttling flow. The connection from the engine to the ACM is illustrated in Figure

\section{3.}

The engine used in this experiment is a six liter Perkins diesel engine. It is a four-stroke, inline six-cylinder engine. This choice of engine platform is relevant to sports car and racing applications because the engine displacement is similar, even though the speed range is lower. This is not necessarily a problem because the engine turbocharger's turbine is sized for the engine in consideration, and in the worst case, it will provide a lower limit for load and speed that the ACM can be effectively operated. Since, in racing applications, the engine is typically at higher load and speed, this is not a concern.

The engine is fully-instrumented and is connected to a dynamometer. The enginedynamometer setup is part of an engine's class, and the sensors are calibrated. A calibration log is maintained, but even so, the sensors were verified to match their previous calibrations.

The Perkins diesel engine was first tested with the stock turbocharger to set a baseline for comparison of the new compressor for the primary, or engine, turbocharger. The second turbocharger is sized to efficiently accommodate the additional air flow required for the operation of the engine and ACM. The new turbocharger has a larger compressor and a variable nozzle turbine.

The experiments performed to assess the effects of ACM operation on engine performance were conducted by running the engine at an operating point allowing maximum boost levels to 
be reached before opening the diverter valve to the ACM. This demonstrates the effect of ACM system air flow requirements on boost level available to the engine and change in fuel consumption, the two primary factors considered in a racing application.

\subsection{ACM Turbine-Compressor Matching}

The matching process for the ACM rotating assembly follows the method presented in Appendix $\mathrm{C}$, but there are some new considerations regarding the ACM application. The turbine and compressors that are paired on production turbochargers are well-matched for gasoline or diesel engine applications, where there is hot exhaust gas driving the turbine. This temperature difference in the engine and ACM applications causes a large change in corrected turbine speed and corrected mass flow rate, two parameters used to map turbine performance. Since the ACM turbine inlet conditions will always be cooler than the original engine application, a larger compressor will be needed to reduce corrected turbine speeds. The compressor matching technique is essentially unchanged, since the compressor conditions are similar in both applications.

\subsection{Primary Compressor Resizing for ACM operation}

Resizing the primary engine turbocharger compressor was required to accommodate the additional airflow required during ACM operation but still has a sufficient surge margin for safe engine operation. The method employed in sizing a new compressor for this project split the operating points symmetrically around the imaginary line that passes through the center of the efficiency contours up the map. This is graphically demonstrated below. 


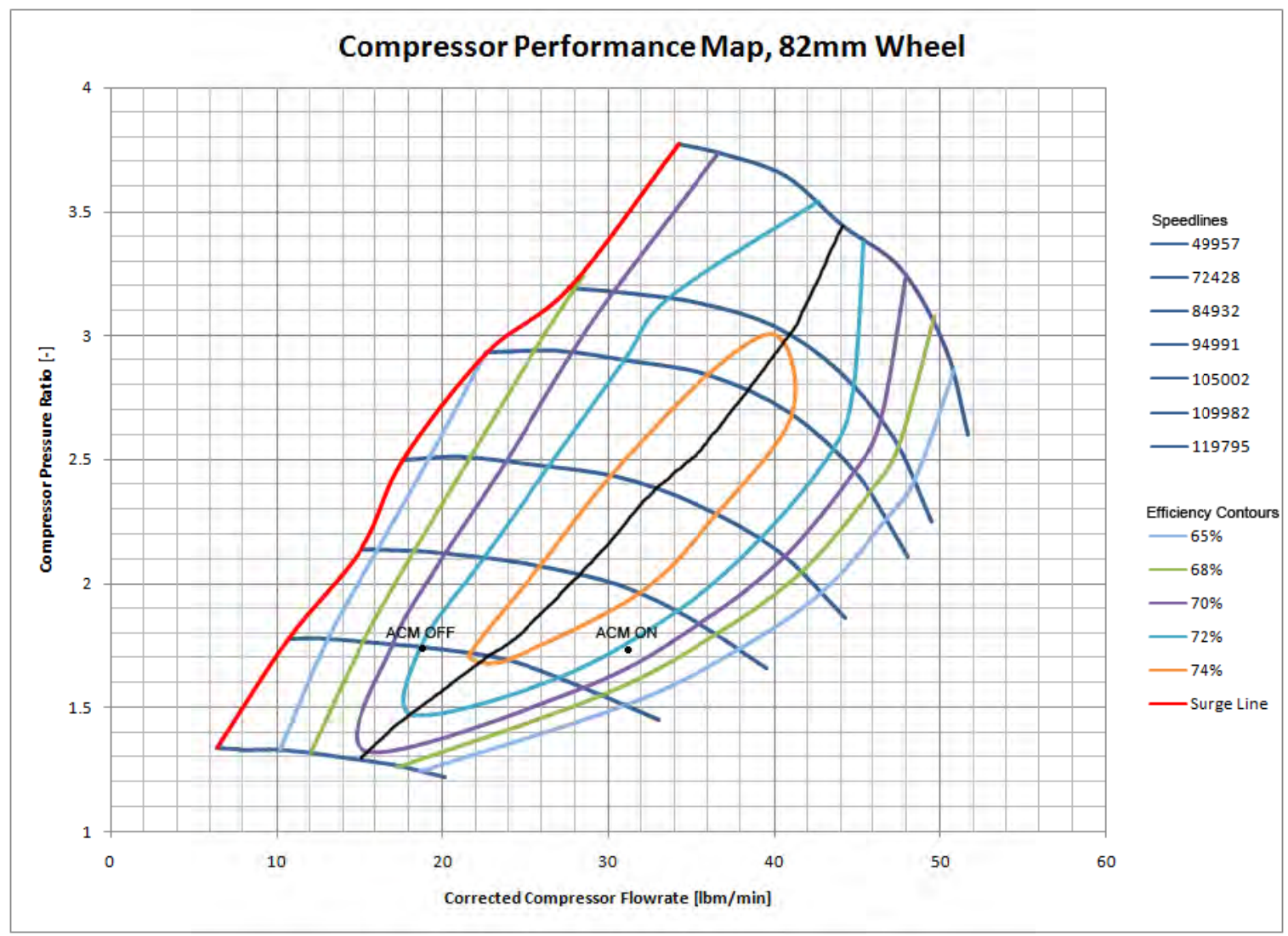

Figure 3.4 Primary engine compressor matching process with an engine operating point of 1600rpm and full load.

Splitting the operating points around the peak efficiency line provides the best average efficiency if the ACM was operated the same amount of time as it was off. This is not likely to be the case. However, since this project does not involve a specific application, the actual duty cycle is unknown and a 50\% duty cycle is assumed. The split on the actual compressor match was not $50 \%$ due to available commercial compressor assemblies. Therefore, the nearest available compressor size was chosen. The primary compressor efficiency remains nearly constant whether or not the ACM is operating by placing the operating point on each side of the nearest efficiency contour. There is opportunity for optimizing the compressor selection process, but detailed, application-specific data is required. The compressor operating boost curves for this project are shown in the figure below. 


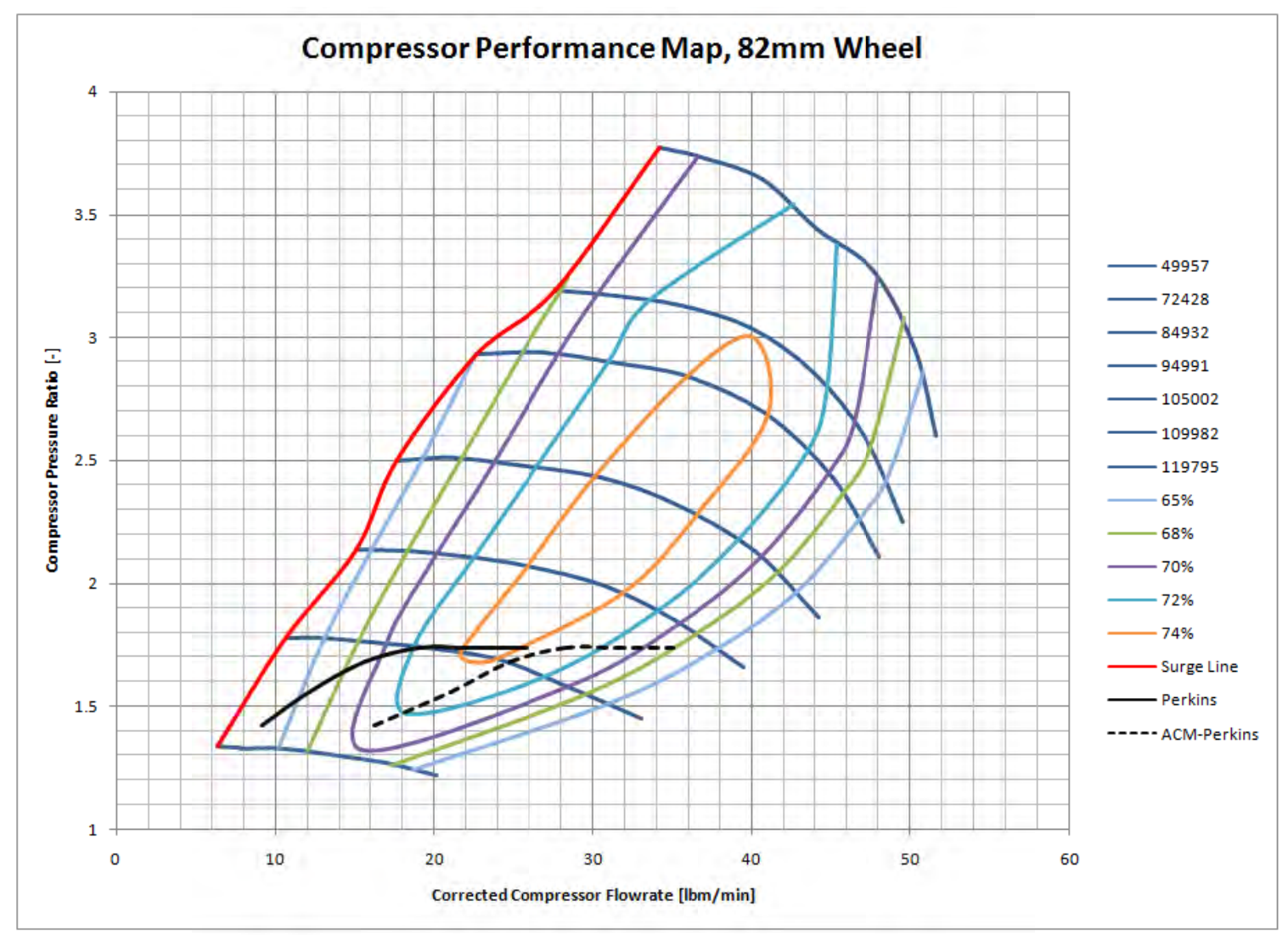

Figure 3.5 Primary engine compressor match with the Perkins diesel boost curve and the target boost curve with the ACM operating.

The compressor selected for the Perkins diesel engine allows for full engine operation with and without the ACM operating. This match provides a sufficient surge margin and low risk of compressor overspeed, while optimizing efficiency as much as possible for this project. 


\section{Chapter 4: Results, Conclusions, and Future Work}

\subsection{Results and Discussion from Unmodified GT1241 Shop Air Test}

\section{Stand}

The unmodified GT1241 turbocharger was used as a performance baseline for comparison of modifications to the turbocharger, and it performed better than expected. The ACM performance is shown in Figures 4.1 and 4.2. An accurate prediction of performance was hard to obtain due to limited compressor and turbine performance data from the manufacturer. This is because the ACM operating conditions, specifically turbine inlet temperature and pressure, caused an increase in corrected turbine speed, compared to an on-engine application. The ACM operating conditions put the target corrected turbine speed outside of the known turbine performance map. However, the increased corrected turbine speed did not cause a sharp turbine efficiency drop, which was likely to occur due to excessive speed from the relatively small compressor. The limiting factor in this configuration was the intercooler, which can be seen in Figure 4.3. The relatively low intercooler effectiveness limited heat removal from the air entering the turbine. This is detrimental to performance as an increase in turbine inlet temperature will generally cause an increase in turbine outlet temperature, decreasing the cooling capacity of the ACM. The data points in Figures 4.1-4.3 were collected simultaneously, so the mass flow rate can be related to the ACM pressure ratio, DAR COP, and DAR cooling capacity. For example, the third point from the right in Figure 4.1 corresponds to the third point from the right in Figure 4.2-4.3. 


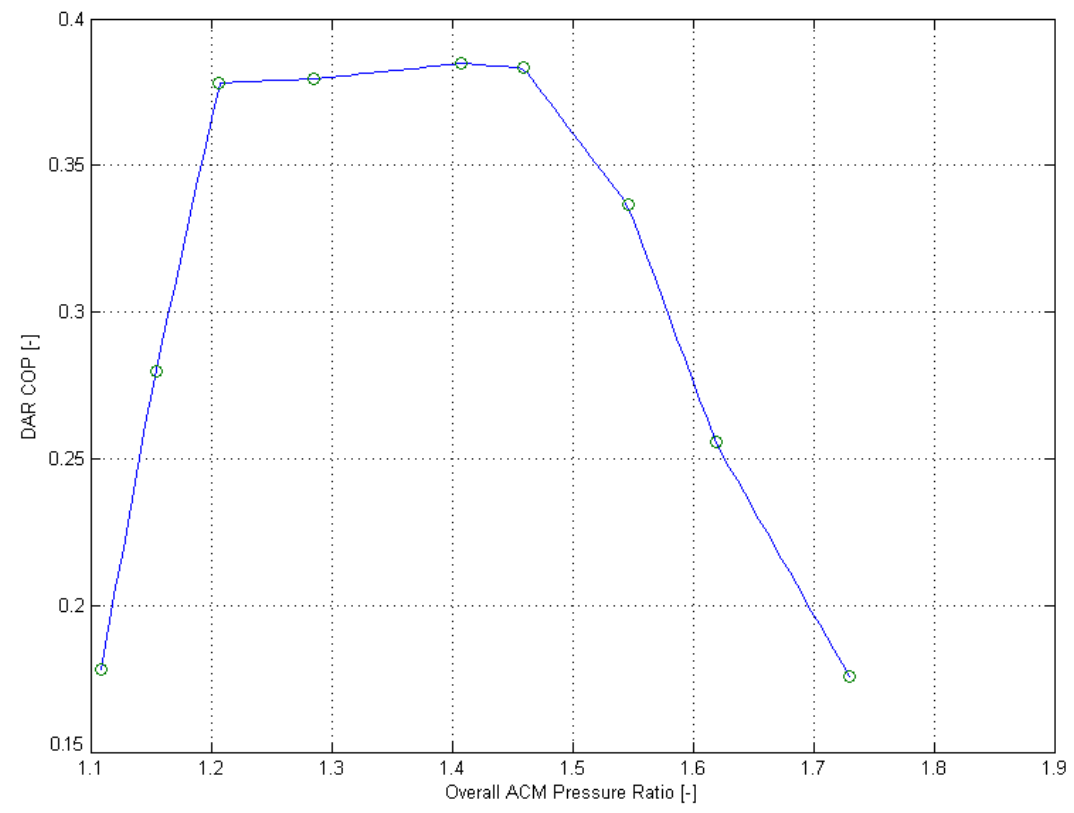

Figure 4.1 Dry air rated COP for the unmodified GT1241 turbocharger. Data points in Figures 4.1-4.3 have a one-to-one correspondence.

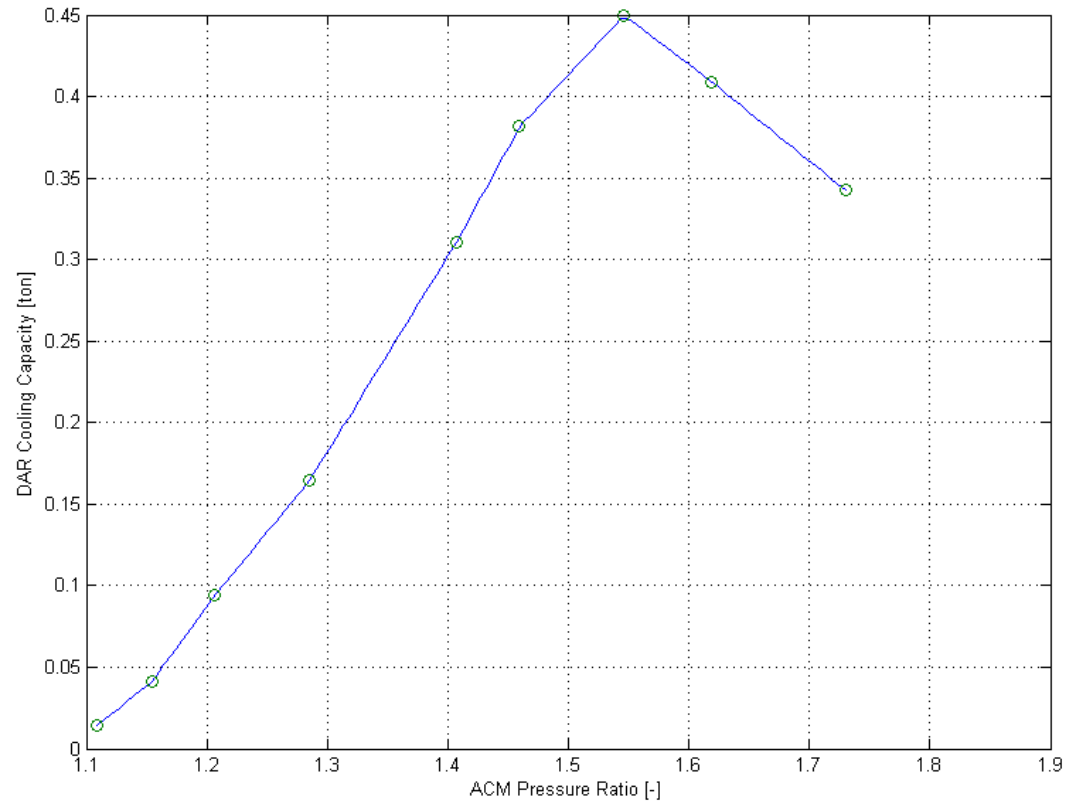

Figure 4.2 Dry air rated ACM cooling capacity. Data points Figures 4.1-4.3 have a one-to-one correspondence. 
The cooling capacity required in a performance automotive application is approximately one ton of cooling. This was determined from reviewing R134a compressor performance maps from Sanden [Appendix H]. This is discussed in greater detail in Section 4.4.

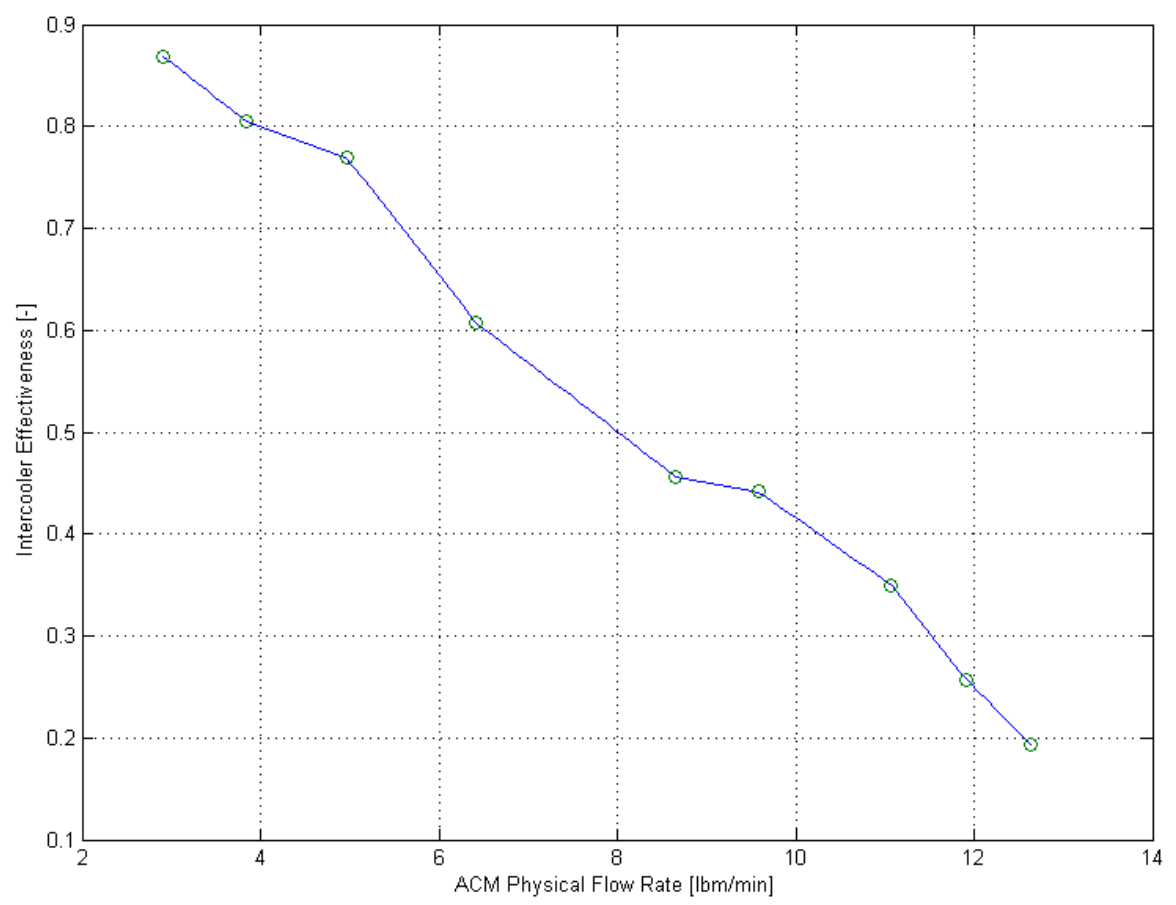

Figure 4.3 Intercooler effectiveness variation with mass flow rate in the GT1241 ACM with the electric fan.

Data points Figures 4.1-4.3 have a one-to-one correspondence.

The ACM intercooler effectiveness was limited because of poor electric fan performance. The heat exchanger performance was shown to have a significant impact on overall ACM performance in Section 2.7, so this was remedied in later tests by manually regulating compressed air to pass over the cold side of the heat exchanger. However, to continue with testing on schedule, the next compressor was installed before the heat exchanger performance was improved. The DAR COP has a peak because as the pressure ratio across the ACM starts at unity and increases, both the ACM compressor and turbine will pass through their peak efficiencies. The DAR cooling capacity will increase until both the compressor and turbine have 
passed their peak efficiencies and begin to decline in performance as the pressure ratio across the ACM is further increased.

The peak dry air rated COP, with the $70 \%$ primary compressor efficiency assumption, is on par with a COP of the theoretical optimization of the ACM from Queen's University, Belfast [3], a COP of 0.62. However, the maximum cooling capacity does not occur at the maximum COP; the dry air rated COP falls to approximately 0.48 at that point.

\subsection{Results and Discussion from Modified GT1244 Shop Air Test Stand}

The initial testing of the GT1244 turbocharger used the same intercooler-fan assembly as the previous test to have a direct comparison of a single component change in the system. The change in the compressor wheel and housing did not change the peak dry air rated COP of the $\mathrm{ACM}$, but it did greatly expand the useful operating range of the ACM and increase the system cooling capacity, pushing it closer to one ton of cabin cooling. This can be seen in Figures 4.44.5. The performance improvements from the compressor wheel and housing change from the unmodified GT1241 configuration are shown below. The intercooler effectiveness is approximately the same as in the unmodified GT1241testing, Figure 4.6. The intercooler is still a limiting factor. However, with its performance approximately the same as in the GT1241 ACM testing, the results still show a relative improvement of the $44 \mathrm{~mm}$ compressor wheel over the previous $41 \mathrm{~mm}$ compressor wheel. The data points were collected simultaneously during this testing, and each point corresponds to the same data point number in the other plots in Figure

\section{4-4.6.}




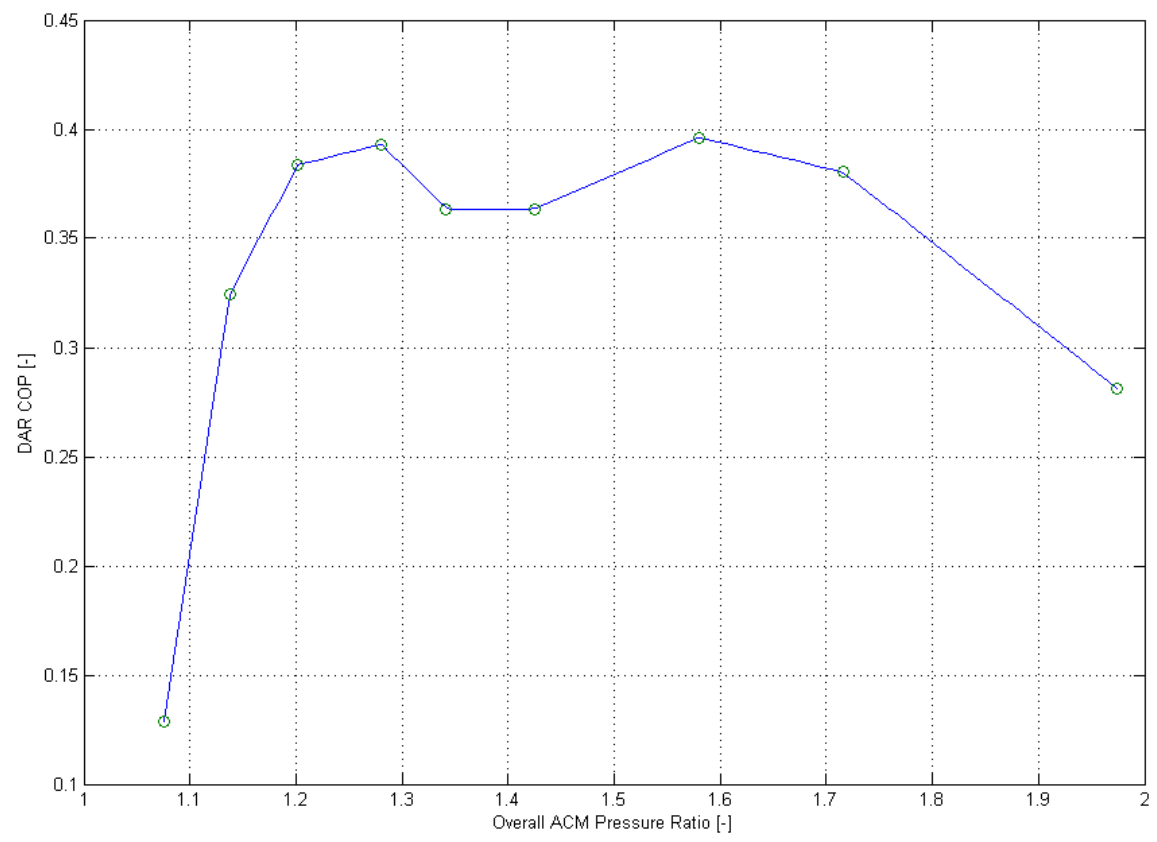

Figure 4.4 Dry air rated COP for the modified GT1244 turbocharger. Data points Figures 4.4-4.5 have a one-to-one correspondence.

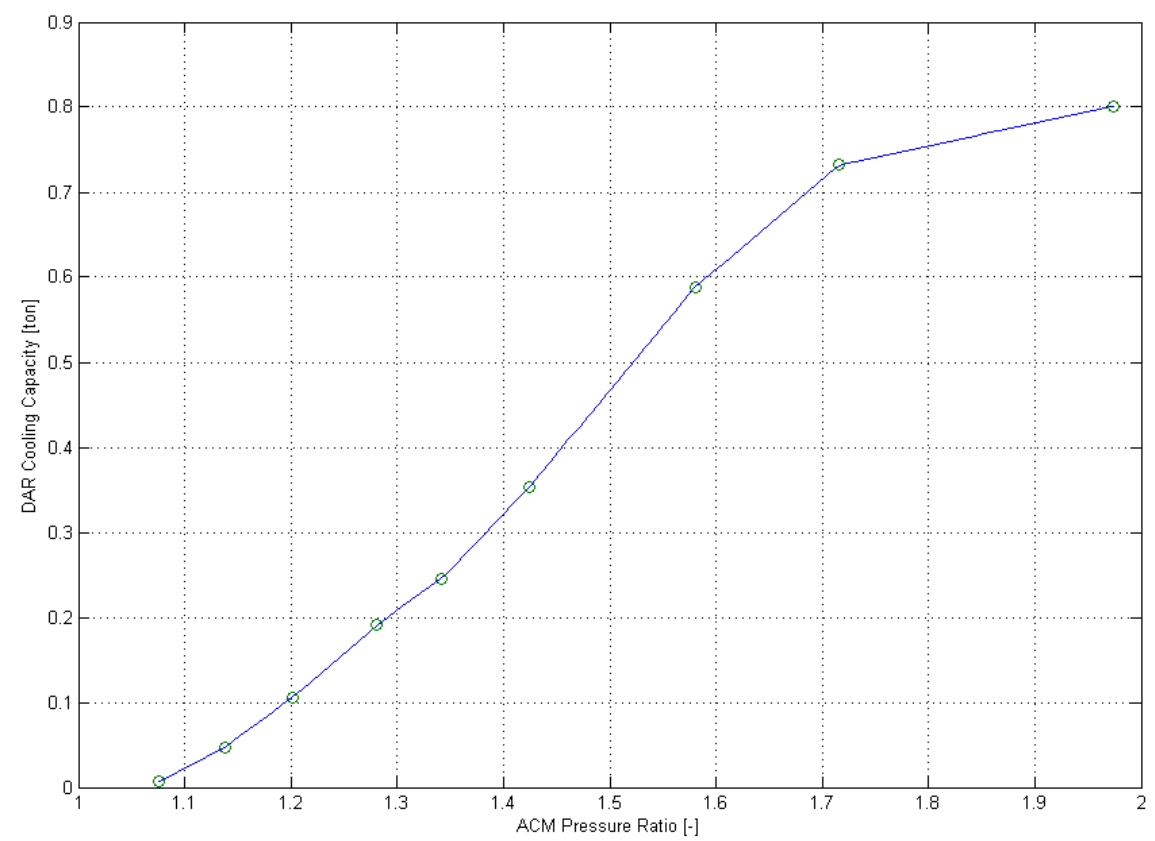

Figure 4.5 Dry air rated ACM cooling capacity. Data points Figures 4.4-4.5 have a one-to-one correspondence. 


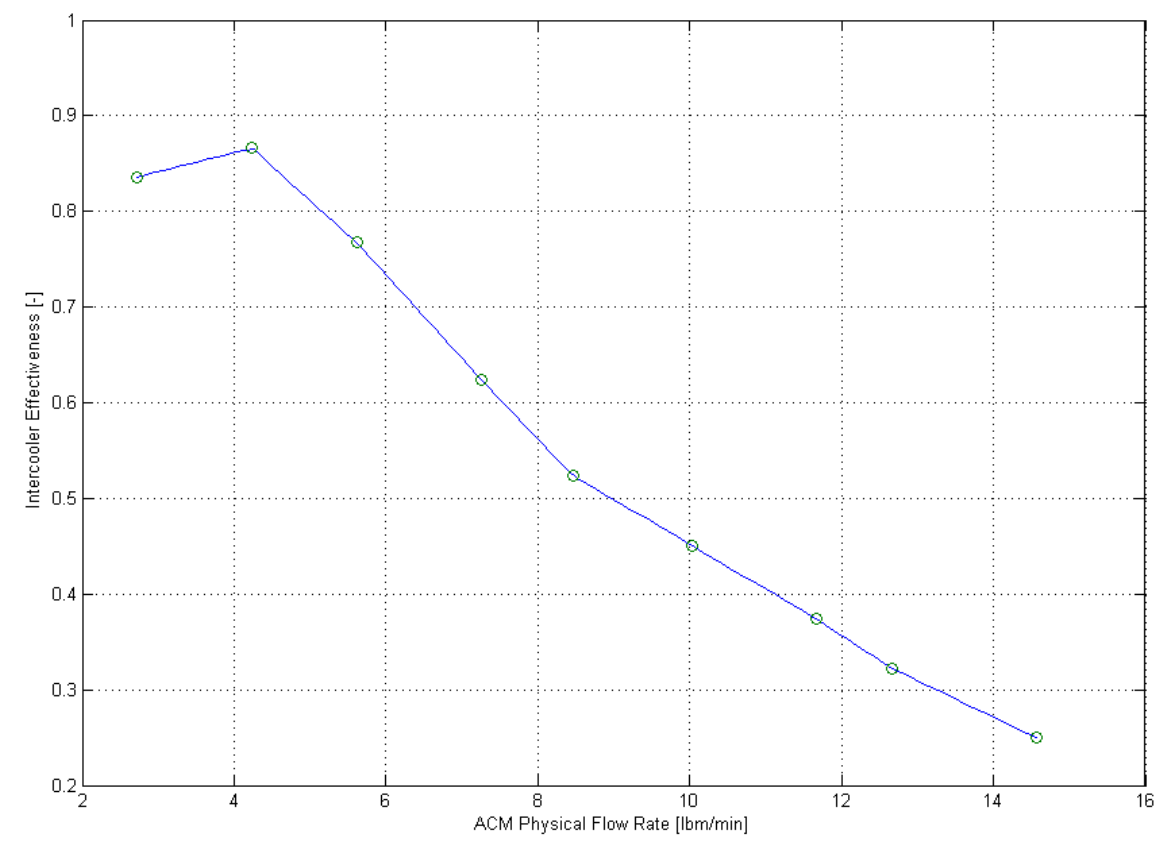

Figure 4.6 Intercooler effectiveness variation with ACM mass flow rate using the electric fan. Data points Figures 4.4-4.5 have a one-to-one correspondence.

Next, the intercooler cold-side airflow problem was fixed. This was achieved by routing manually-regulated compressed air over the cold side. The airflow over the cold side of the intercooler was kept within reasonable levels that could be obtained with a typical configuration seen in automotive applications. This was determined to be approximately 350 CFM, and this agrees with volumetric flow rates observed in R134a condensers [9]. Based on intercooler cold flow entrance area, this results in an average air velocity of approximately $8.5 \mathrm{ft} / \mathrm{s}$ entering the face of the intercooler core. This is even easier to achieve when the vehicle would be moving. The results from the improved intercooler configuration can be seen below. The intercooler performance increase improved both ACM COP and DAR cooling capacity, Figure 4.7-4.8. The increase in intercooler effectiveness can be seen in Figure 4.9. The data points were collected 
simultaneously during this testing, and each point corresponds to the same data point number in the other plots in Figure 4.7-4.9.

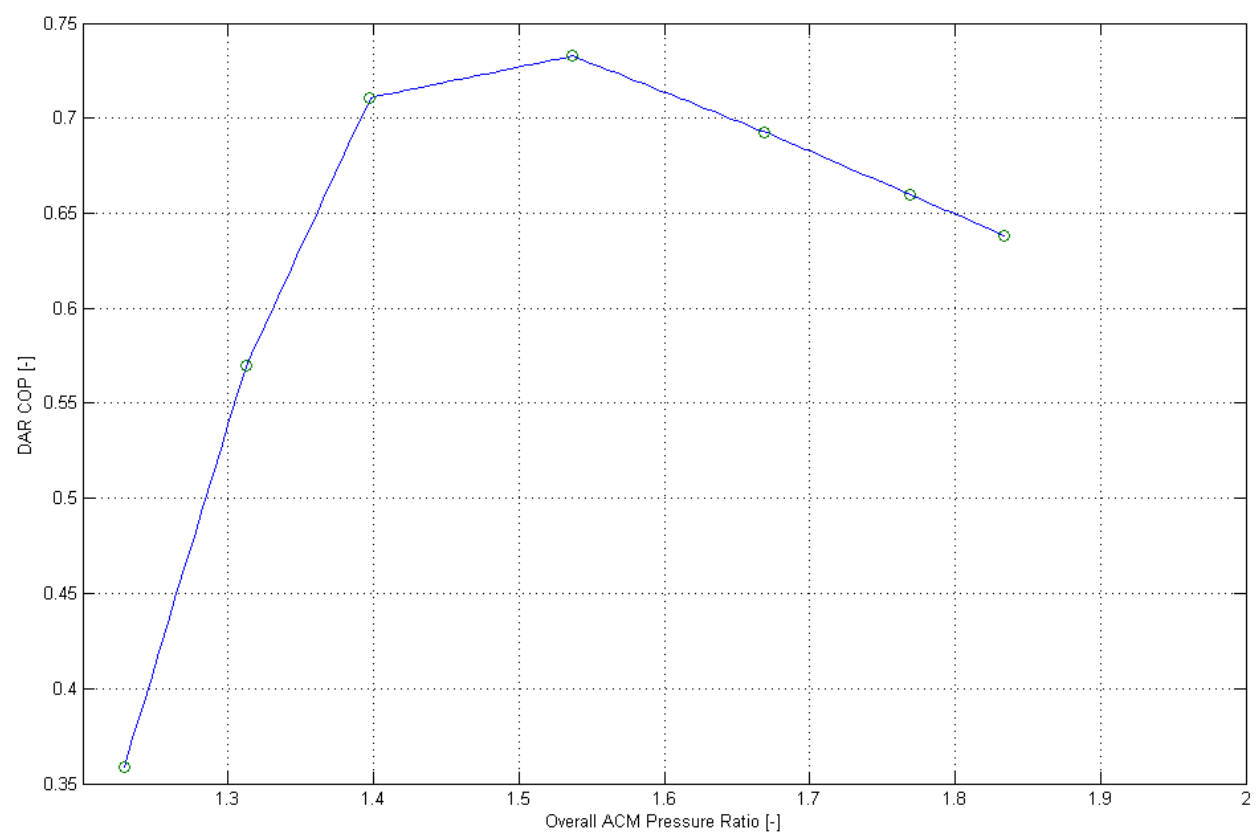

Figure 4.7 Dry air rated COP for the modified GT1244 turbocharger. Data points Figures 4.7-4.9 have a one-to-one correspondence. 


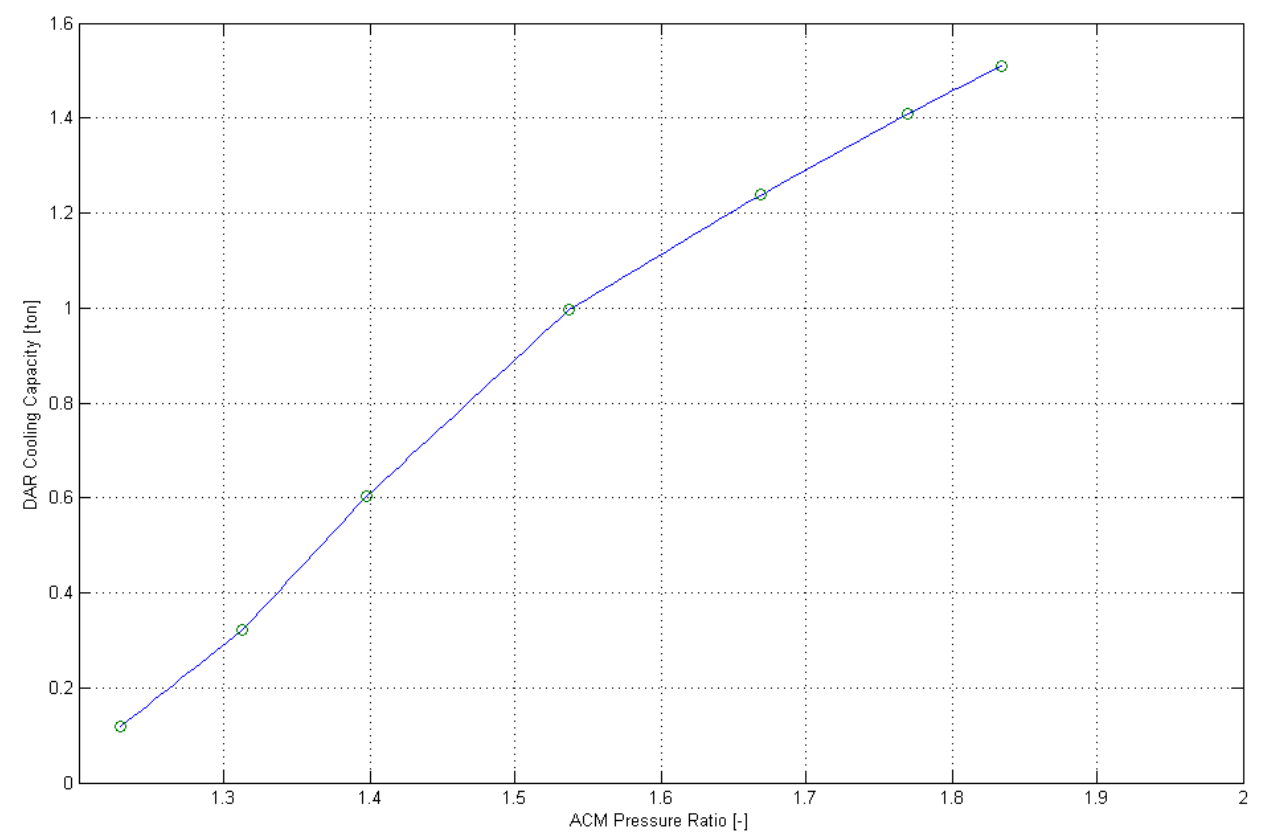

Figure 4.8 Dry air rated ACM cooling capacity. Data points Figures 4.7-4.9 have a one-to-one correspondence.

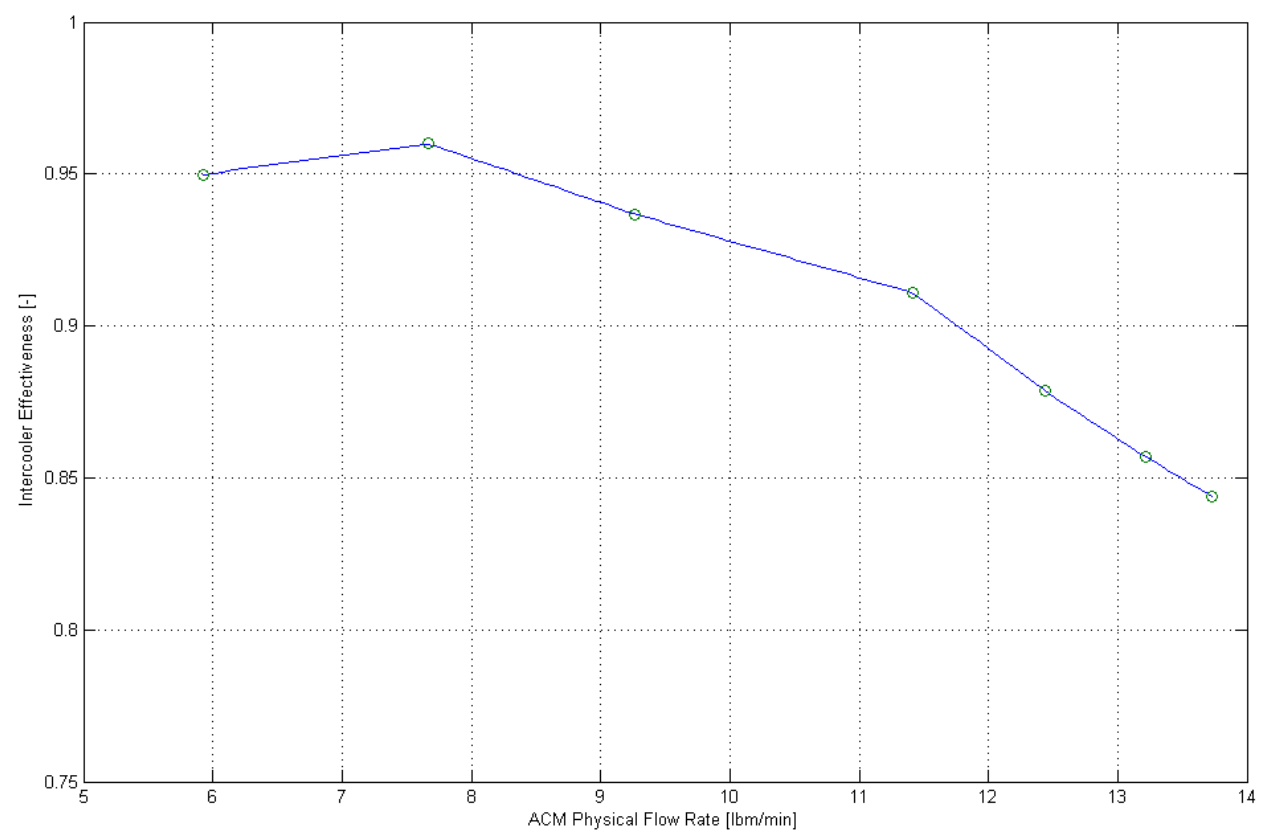

Figure 4.9 Intercooler effectiveness variation with ACM mass flow rate. Data points Figures 4.7-4.9 have a one-to-one correspondence. 
The ACM intercooler proved to be a critical component in overall ACM performance. The improved intercooler effectiveness approximately doubled the dry air rated COP and cooling capacity.

The theoretical performance analysis was shown to be a good approximation of the actual performance of the ACM. The flow prediction from the compressor-turbine match and the turbine pressure ratio-flow profile and actual flow conditions are over-plotted on the compressor performance map for the $44 \mathrm{~mm}$ compressor wheel. At lower pressure ratios, all measurements and corresponding predicted values agree within $7 \%$ difference or better. At higher pressure ratios across the ACM the dry-air rated COP and DAR cooling capacity calculations both matched actual performance within approximately $13 \%$, but the mass flow rate deviates more. The mass flow rate deviates up to $12 \%$ difference from the measured mass flow rate. At this point, the pressure ratio across the ACM is approximately 1.84 . The results of the compressor operating points comparison can be seen in Figure 4.10. The DAR cooling capacity and DAR COP comparisons agree well and are shown in Table 4.2. 


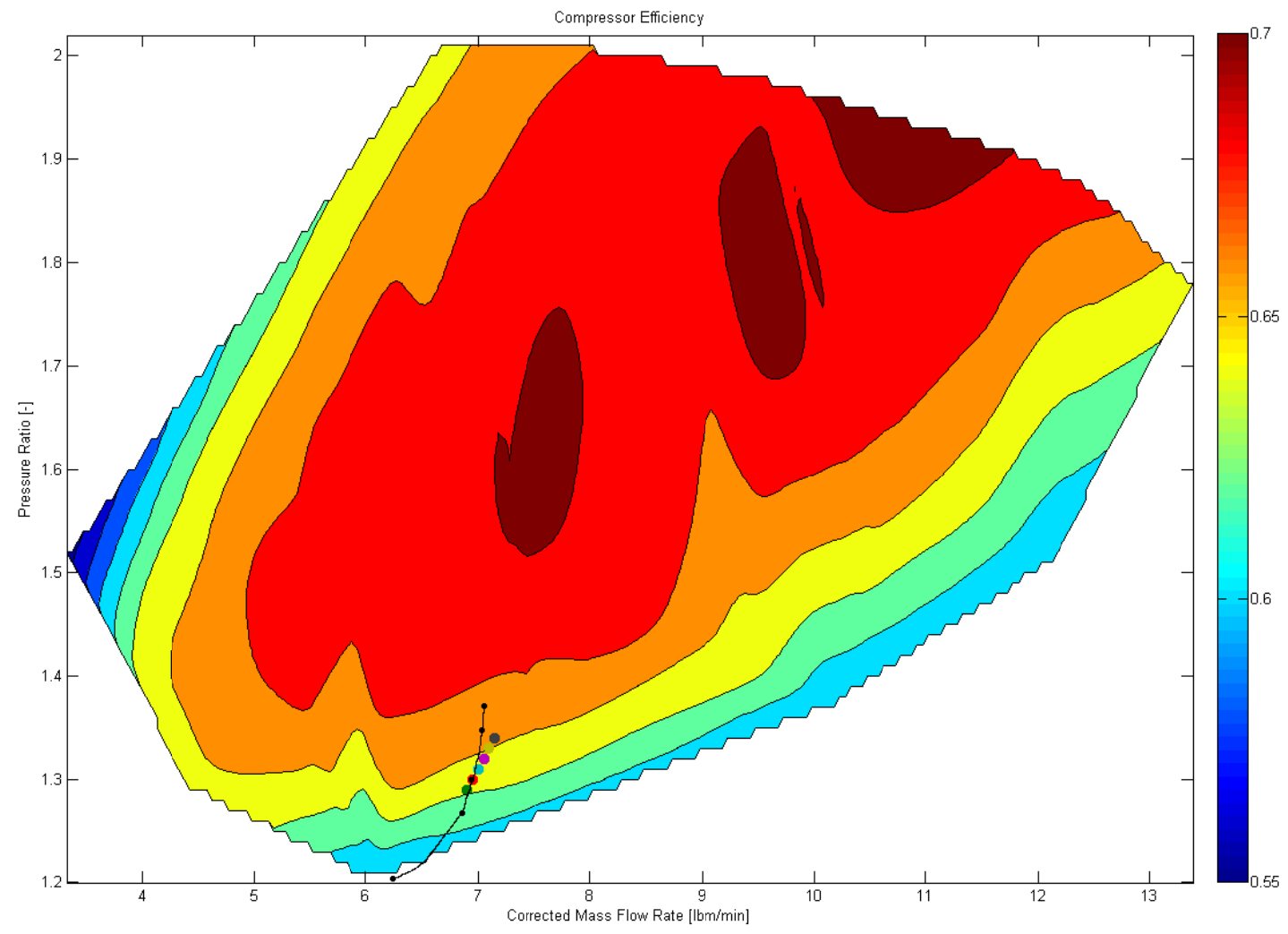

Figure 4.10 Locus of theoretical and actual operating points over-plotted on GT1244 compressor map.

Since the operation of the ACM placed the operation of the ACM at the outer limits of performance data collected by the turbocharger manufacturer, limited speed, power, and mass balance points could be obtained. If more component performance data was available, a more complete locus of operating points could be obtained with the computer simulation. Even with limited data, the part of the locus of operating points that was obtained from the computer simulation matches well with the experimental data. The computer simulations, in both Matlab and Engineering Equation Solver (EES), use the equations derived in Appendix B and the matching process described in Appendix C. A comparison of the theoretical and actual 
operation using the conditions specified in Table 4.1 results in the performance presented in Table 4.2.

Table 4.1 Operating Conditions for single point comparison of theoretical and actual performance.

\begin{tabular}{|c|c|c|c|}
\hline Ambient and Operating Conditions & Value & $\underline{\text { Units }}$ & Uncertainty [+/-] \\
\hline Ambient Temperature & 531 & [Deg. R] & 1.7 \\
\hline Ambient Pressure & 14.69 & [psia] & 0.02 \\
\hline Ambient Relative Humidity & 0.4 & {$[-]$} & 0.08 \\
\hline ACM Compressor Inlet Temperature & 526 & [Deg. R] & 1.7 \\
\hline ACM Compressor Inlet Pressure & 26.95 & [psia] & 0.02 \\
\hline ACM Compressor Efficiency & 0.72 & {$[-]$} & 0.02 \\
\hline ACM Compressor Outlet Pressure & 37.08 & [psia] & 0.02 \\
\hline ACM Intercooler Effectiveness & 0.83 & {$[-]$} & 0.04 \\
\hline ACM Intercooler Pressure Drop & 0.3 & [psi] & 0.03 \\
\hline ACM Mass Flow Rate & 13.73 & [lbm/min] & 0.6 \\
\hline ACM Turbine Inlet Temperature & 536 & [Deg. R] & 1.7 \\
\hline ACM Turbine Efficiency & 0.56 & {$[-]$} & 0.02 \\
\hline
\end{tabular}

Table 4.2 Results of single point comparison of theoretical and actual performance.

Performance Parameter

COP, Dry Air Rated [-]

Cooling Capacity, Dry Air Rated [ton]

Theoretical
Operation
0.61

1.7
Actual Operation 0.64

1.5

\section{Uncertainty [+/-] 0.04 \\ 0.06}

\subsection{Results and Discussion from GT1244 On-Engine Testing}

The on-engine testing was limited because of a bearing failure in the ACM turbocharger.

However, one operating point was tested before the failure occurred. The testing that was completed before failure was still a success with good performance. The COP based on change in bsfc and fuel flow rates indicated a COP of 0.56 , with a corresponding 0.72 tons of cooling capacity. The theoretical optimization of the ACM from Queen's University, Belfast had a COP of 0.62 , while actual performance being much less $[2,3]$. Their actual performance number was not stated, other than being less than expected. The COP for the on-engine testing for this 
project is assuming the ACM is running at a time the engine's turbocharger would not have been waste-gating, otherwise the COP is essentially infinite because the potential power would have been wasted in the turbine bypass free expansion process.

The testing conducted with the ACM being powered by the Perkins diesel's turbocharger had reduced performance from the shop air test stand. The DAR cooling capacity decreased from approximately 0.90 to 0.72 tons, and the DAR COP decreased from approximately 0.73 to 0.56 for corresponding operating points of the shop-air testing of the GT1244 and the on-engine testing. This is due to much higher ACM inlet temperatures coming from the intake manifold of the engine. The on-engine testing conditions represent real world operation more accurately than the shop air test stand.

\subsection{Comparison of ACM to R134a Systems}

The target cooling capacity for the ACM was determined by observing the average cooling capacity based on the Sanden performance charts of the SD5H09 R134a system compressors [Appendix H]. This performance chart indicates the cooling capacity of a typical R134a system using the Sanden compressor. The cooling capacity will vary with suction superheat and operating pressures. The average range was selected from the Sanden chart to represent the target cooling capacity. This is approximately one ton of cooling capacity (12000 BTU/hr). The Sanden performance chart was used because Sanden is a worldwide manufacturer of automotive air conditioning compressors, used in all types of vehicles [10] and would create a good approximation of the cooling requirements for a high-performance vehicle. While the GT1244 ACM fell a little bit short of the cooling capacity goal, the R134a systems are pre-charge pressure and engine speed sensitive. Their actual capacity varies somewhat and are seldom used at full duty cycle at all times (i.e. cabin fan speed and temperature shutoff switch). 
The system weight for a typical R134a system varies between 60-75 $\mathrm{lbf}^{1}$. The ACM system weight and estimated bracket weights totals at $40 \mathrm{lbf}$. This is approximately a $40 \%$ weight reduction. The physical size and number of components of the system is smaller than compared to R134a or Freon systems [1].

Table 4.3 ACM component weight breakdown.

\begin{tabular}{ll} 
Component & Weight [lbf] \\
\cline { 2 - 2 } Turbocharger Assy. & 10 \\
Intercooler Core & 10 \\
IC end caps & 4 \\
electric fan & 5.6 \\
ducting & 2.5 \\
Clamps & 1 \\
silicone connectors & 2 \\
Brackets (for vehicle) & 5 \\
& \\
Total & 40
\end{tabular}

Another aspect of the ACM that differs from typical R134a air conditioning systems is that the ACM does not always induce a parasitic loss on the engine crankshaft, as opposed to a beltdriven R134a compressor. This can be seen on a P-v diagram for a four-stroke engine.

\footnotetext{
${ }^{1} 2008$ e-mail to Vintage Air; unreferenced
} 


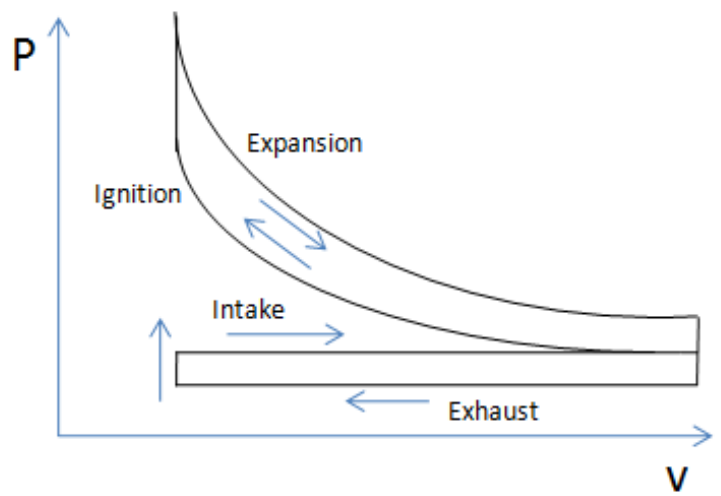

Figure 4.11 P-v diagram for four-stroke gasoline engine.

The lower loop on the P-v diagram is an indicator of the pumping losses on the engine. The pumping losses are the area under the exhaust line minus the area under the intake line in Figure 4.11 or Figure 1.1. It should be noted that in Figure 1.1, the exhaust pressure is greater than the intake pressure. A positive lower-loop loss is typical of naturally-aspirated engines. In Figure 4.11 the net pumping losses are negative, indicating that a forced-induction system is creating a higher engine intake manifold pressure than exhaust backpressure. Turning the R134a system compressor on will induce a load on the engine and always hurt engine performance. On the other hand, a turbocharger can actually create a higher intake pressure than exhaust back pressure. This is due to the turbine recovering wasted exhaust heat, with some exhaust backpressure, providing enough work to the compressor to deliver higher than exhaust pressures to the engine's intake manifold. This helps to minimize engine bsfc. 


\subsection{Conclusion}

The shop-air stand testing successfully demonstrated that an ACM can be designed around automotive turbocharger components. The use of automotive turbocharger components is critical to reducing cost in development of commercial systems for automotive use. An off-theshelf turbocharger can be used in certain applications without modification and provide adequate performance. The testing on the unmodified GT1241 turbocharger indicated a peak DAR COP of approximately 0.38 , and a DAR cooling capacity of 0.45 tons. The performance was somewhat limited due to the intercooler effectiveness initially, in the first two experiments of the GT1241 and GT1244, before the heat exchanger issue was remedied. It is expected that if the intercooler effectiveness was increased with this turbocharger configuration, the performance would be significantly increased, as seen in the modified GT1244 testing.

The GT1244 was optimized over the baseline GT1241 turbocharger. The ACM turbine and compressor were selected for the ACM operating conditions, as opposed to the typical onengine application these turbochargers are normally used for. Initially, the peak DAR COP and DAR cooling capacity was approximately the same as the baseline GT1241 configuration. However, even with the intercooler performance being limited, the operating range was significantly increased (Figure 4.4-4.5). Once the intercooler effectiveness was increased by providing adequate airflow across the cold side of the heat exchanger core, the peak DAR COP reached 0.73 with a peak DAR cooling capacity of 1.5 tons (Figure 4.7-4.9). The DAR COP figures for the shop-air testing were determined by assuming a primary compressor efficiency of $70 \%$. This primary compressor efficiency is typical of modern turbochargers in application. For reference, the compressor used for the on-engine testing in this project has an isentropic efficiency of $70 \%$, or greater at the target boost pressure of $11 \mathrm{psig}$, over a mass flow range of approximately $16-32 \mathrm{lbm} / \mathrm{min}$. 
The on-engine testing has shown that an air-cycle air conditioning system can be effective for racing applications, where there is plenty of opportunity to take advantage of time that would have otherwise been spent waste-gating. The testing performed prior to the ACM thrust bearing failure indicated a DAR COP of 0.56 and DAR cooling capacity of 0.72 tons. This operating point was not at full boost pressure due to the lower engine load and speed operating point initially tested, and the full benefit of the setup, operation during typical waste-gating conditions, could not be tested.

In comparison to previous work at Queen's University, Belfast, the ACM in this project performed well. The theoretical COP of the previous work was $0.294[1,2]$. They performed a theoretical optimization of the same system with an expected COP of 0.62 . The theoretical optimization used compressor and turbine efficiencies of state-of-the-art components. However, their final results from actual testing were not presented other than a short statement saying that fuel consumption of the engine increased over three times the amount for the vapor-compression cycle they implemented as well. The group attributed the less-thanexpected performance to excess losses in components such as the ACM bearings and primary compressor on the engine.

If the ACM bearing failure had not occurred, the next operating point would have been at higher load and speed to provide a better comparison to the waste-gated turbocharger operation. The operating point tested corresponds to a point where the stock turbocharger was not yet wastegating. This means that the boost pressure can be increased to the original limit, resulting in increased cooling capacity due to increased pressure ratio across the ACM. Also, the effects on engine performance can be decreased because the ACM will be consuming a smaller fraction of the overall engine airflow. At larger engine loads and speeds, there is significant excess turbine 
power that would normally be limited by waste-gate operation. Factors that influence turbine power are turbine mass flow rate, exhaust gas temperature, and pressure ratio across the turbine. Both turbine mass flow rate and exhaust gas temperature typically increase with engine load and speed. With this in mind, engine operating points corresponding to waste-gate operation are expected to have increased DAR COP following the proposed definition of $\mathrm{COP}_{\text {Effective }}$ in Eqn. 2.2.

The engine turbocharger matching criteria has potential to be further optimized for specific applications. This will minimize effects of ACM operation on engine performance. The nominal ACM mass flow rate relative to the engine mass flow rate at typical engine operating conditions is an important consideration because too large of an ACM and the primary compressor will not be able to maintain desired boost pressures at higher combined system mass flow rates. The air flow to the ACM can be throttled, but this will reduce the potential cooling capacity of an ACM being designed for the vehicle. While throttling the ACM may be necessary is some engine operating conditions, it is not optimal for performance and should be avoided as much as possible.

\subsection{Future Work}

While there is a considerable amount of work to still be done in designing an ACM for production, most of it can be easily handled by a turbocharger manufacturer. The conditions the ACM turbocharger compressor is operating in are similar to a second turbo in a series turbocharger setup used on modern diesel engines to more efficiently handle increased boost pressures. The main consideration is the bearing system for the ACM. The bearing system experiences higher thrust loading because it needs a larger compressor wheel relative to the turbine wheel. This is because the compressor inlet sees higher than atmospheric pressures 
while the turbine still exits to ambient pressure, and with a larger compressor wheel, there is increased area for the inlet pressure to act upon. Stability of the rotating assembly is the first priority. However, the bearing system efficiency is increasingly critical in low-temperature applications because there is less power being transmitted by the shaft, and the same amount of bearing drag will now be a larger fraction of the power transmitted. To increase stability and decrease bearing losses, magnetic or air bearings may be considered $[5,7]$.

The ACM turbocharger can operate in a smaller range than a typical on-engine application, and this allows for more optimization of the compressor and turbine designs for higher peak efficiency at the cost of usable mass flow rate range [8]. Once a particular application is welldefined, the compressor and turbine can be designed to operate in a narrower range that is adequate for the application. This requires a considerable amount of research and development and would only be practical for larger production quantities.

To completely prove out the concept with specific packaging requirements, an in-vehicle demonstration would be ideal. The type of vehicle could be anything performance-oriented with a medium to large engine, due to the minimize size of turbochargers available for ACM design. The turbocharger used for the ACM in this project is the smallest commercially available from Garrett Turbochargers. The in-vehicle demonstration would allow for an operation dutycycle to be recorded and facilitate in optimizing the engine turbocharger's compressor matching process. 


\section{References}

1. Scofield, Paul C. (1949).Air Cycle Refrigeration. Refrigerating Engineers, 57, [558-563, 611612].

2. Spence, Stephen W.T., Doran, W. John, \& Artt, David W. (2004). Design, construction and testing of an air-cycle refrigeration system for road transport. International Journal of Refrigeration, 27, 503-510.

3. Spence, Stephen W.T., Doran, W. John, Artt, David W., \& McCullough, G. (2005). Performance analysis of a feasible air-cycle refrigeration system for road transport. International Journal of Refrigeration, 28, 381-388.

4. Arora, C P. (2000). Refrigeration and air conditioning. West Patel Nagar: Tata McGraw-Hill Publishing Company Limited.

5. Murray, Charles J. (1994).Magnetic Bearing Improves Air Cycle Cooling Reliability. Design News, 49, 85-86.

6. Turbo Tech 101. Retrieved from http://www.turbobygarrett.com/turbobygarrett/tech_center/turbo_tech101.html

7. Burgmeler, Lyman, \& Poursaba, Matt (1994). Ceramic Hybrid Bearings in Air-Cycle Machines. International Gas Turbine and Aeroengine Congress and Exposition, 94-GT-393, 1-9.

8. Dresser-Rand, Olean \& Wellsville Operations. (2006). Range Versus Efficiency - A Dilemma for Compressor Designers and Users [Brochure]. Olean, NY: James M. Sorokes.

9. Hosnoz, M., \& Direk, M. (2006). Performance evaluation of an integrated automotive air conditioning and heat pump system. Energy Conversion \& Management, 47, 545-559.

10. Retrieved from www.sanden.com 


\section{Appendix A - Uncertainty Analysis}

Uncertainty of a dependent variable $F(x, y, z)$, in general can be calculated as:

$$
u_{F}=\left[\left(\frac{\partial F}{\partial x} u_{x}\right)^{2}+\left(\frac{\partial F}{\partial y} u_{y}\right)^{2}+\left(\frac{\partial F}{\partial z} u_{z}\right)^{2}\right]^{1 / 2}
$$

where $u_{F}$ is the uncertainty in the parameter of interest, such as compressor efficiency, and $u_{x}$, $\mathrm{u}_{\mathrm{y}}$, and $\mathrm{u}_{\mathrm{z}}$ are the measurement uncertainties.

Table A.1 Measurement Uncertainty Based on Calibration Instrumentation

\begin{tabular}{|c|c|}
\hline Measurement & Measurement Uncertainty \\
\hline Pressure & 0.02 [psi] \\
\hline Temperature & $1.7[$ Deg. F] \\
\hline Volumetric Flow Rate & approx. 5 [CFM] \\
\hline
\end{tabular}

\section{A.1 Compressor Efficiency}

The compressor efficiency uncertainty was calculated using conditions representative of ACM operating conditions. In doing so some assumptions are required. It is assumed the compressor and turbine efficiency are $70 \%$ and the intercooler effectiveness is $70 \%$.

The compressor efficiency can be calculated as:

$$
\eta_{C}=\frac{T_{1 c}\left(P R_{c}^{\gamma_{c}-1 / \gamma_{c-1}}\right)}{T_{2 c}-T_{1 c}} \quad(\text { Eqn. A.2) }
$$

The partial derivates are calculated:

$$
\begin{gathered}
\frac{\partial \eta_{c}}{\partial T_{1 c}}=\frac{\left[\left(\frac{P_{2 c}}{P_{1 c}}\right)^{\gamma_{c}-1 / \gamma_{c}}\right]}{\left(T_{2 c}-T_{1 c}\right)}+\frac{T_{1 c}\left[\left(\frac{P_{2 c}}{P_{1 c}}\right)^{\gamma_{c}-1 / \gamma_{c}}\right]}{\left(T_{2 c}-T_{1 c}\right)^{2}} \quad \text { (Eqn. } \\
\frac{\partial \eta_{c}}{\partial T_{2 c}}=-\frac{T_{1 c}\left[\left(\frac{P_{2 c}}{P_{1 c}}\right)^{\gamma_{c}-1 / \gamma_{c}}\right]}{\left(T_{2 c}-T_{1 c}\right)^{2}} \quad \text { (Eqn. A.4) } \\
\frac{\partial \eta_{c}}{\partial P_{1 c}}=-\frac{T_{1 c}\left(\frac{P_{2 c}}{P_{1 c}}\right)^{\gamma_{c}-1 / \gamma_{c}}}{P_{1 c}\left(T_{2 c}-T_{1 c}\right)} \frac{\gamma_{c}-1}{\gamma_{c}} \quad \text { (Eqn. A.5) }
\end{gathered}
$$




$$
\frac{\partial \eta_{c}}{\partial P_{2 c}}=\frac{T_{1 c}\left(\frac{P_{2 c} c}{P_{1 c}}\right)^{\gamma_{c}-1 / \gamma_{c}}}{P_{2 c}\left(T_{2 c}-T_{1 c}\right)} \frac{\gamma_{c}-1}{\gamma_{c}} \quad \text { (Eqn. A.6) }
$$

Equations A.3-6 can be used to find the total uncertainty in efficiency by,

$$
u_{\eta_{c}}=\sqrt{\left(\left[\frac{\left.\left(\frac{\left(P_{2 c}\right.}{P_{1 c}}\right)^{\gamma_{c}-1} / \gamma_{c}\right]}{\left(T_{2 c}-T_{1 c}\right)}+\frac{T_{1 c}\left[\left(\frac{P_{2 c}}{P_{1 c}}\right)^{\gamma_{c}-1} / \gamma_{c}\right.}{\left(T_{2 c}-T_{1 c}\right)^{2}}\right)^{2} u_{T_{1 c}}^{2}+\left(-\frac{T_{1 c}\left[\left(\frac{P_{2 c}}{P_{1 c}}\right)^{\gamma_{c}-1 / \gamma_{c}}\right]}{\left(T_{2 c}-T_{1 c}\right)^{2}}\right)^{2} u_{T_{2 c}}^{2}+\left(-\frac{T_{1 c}\left(\frac{P_{2 c}}{P_{1 c}}\right)^{\gamma_{c}-1 / \gamma_{c}}}{P_{1 c}\left(T_{2 c}-T_{1 c}\right)} \frac{\gamma_{c}-1}{\gamma_{c}}\right)^{2} u_{P_{1 c}}^{2}+\left(\frac{T_{1 c}\left(\frac{P_{2 c}}{P_{1 c}}\right)^{\gamma_{c}-1 / \gamma_{c}}}{P_{2 c}\left(T_{2 c}-T_{1 c}\right)} \frac{\gamma_{c}-1}{\gamma_{c}}\right)^{2}\right.}
$$

\section{(Eqn. A.7)}

The results for uncertainty in compressor efficiency are:

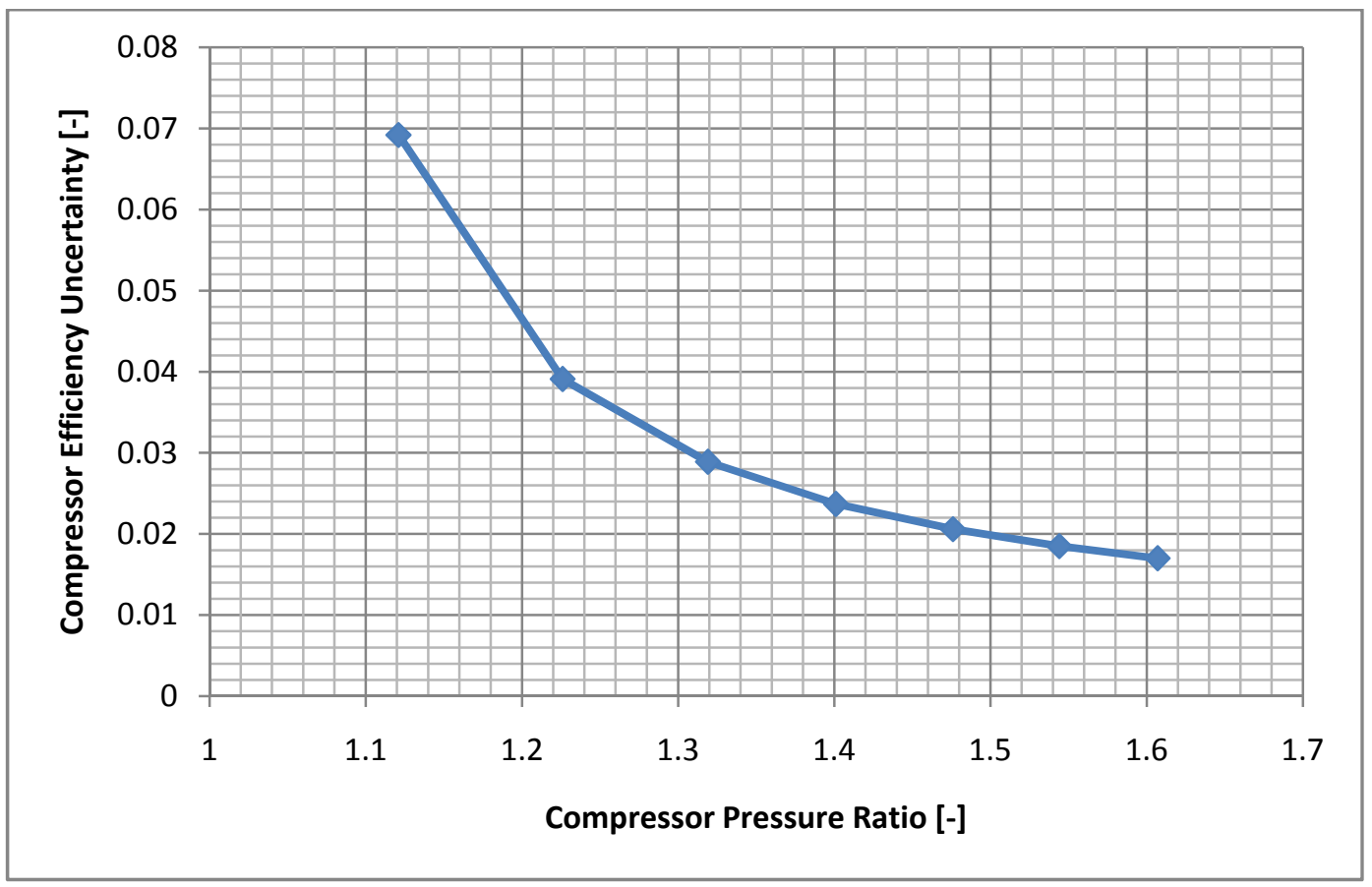

Figure A.1 Uncertainty in ACM compressor efficiency during typical operating conditions.

The uncertainty grows large at low compressor pressure ratios. The calculations are mainly limited by the measurement uncertainty associated with the thermocouples. With low pressure ratios across the compressor, there will be small changes in temperature, and the uncertainty in temperature measurements will be a larger fraction of the temperature difference across the compressor. 
Table A.2 Values used in calculating uncertainty in ACM compressor efficiency calculations.

\begin{tabular}{|r|r|r|r|r|r|}
\hline $\begin{array}{c}\text { Compressor } \\
\text { Inlet } \\
\text { Pressure } \\
\text { [psia] }\end{array}$ & $\begin{array}{c}\text { Compressor } \\
\text { Outlet } \\
\text { Pressure } \\
\text { [psia] }\end{array}$ & $\begin{array}{c}\text { Compressor } \\
\text { Inlet } \\
\text { Temperature } \\
\text { [Deg. R] }\end{array}$ & $\begin{array}{c}\text { Compressor } \\
\text { Outlet } \\
\text { Temperature } \\
\text { [Deg. R] }\end{array}$ & $\begin{array}{c}\text { Compressor } \\
\text { Efficiency } \\
\text { Uncertainty } \\
{[-]}\end{array}$ & $\begin{array}{c}\text { Compressor } \\
\text { Pressure } \\
\text { Ratio [-] }\end{array}$ \\
\hline 16.69 & 18.71 & 530 & 555.1 & 0.0692 & 1.121 \\
\hline 18.69 & 22.92 & 530 & 575.3 & 0.0391 & 1.226 \\
\hline 20.69 & 27.29 & 530 & 592.1 & 0.0289 & 1.319 \\
\hline 22.69 & 31.8 & 530 & 606.5 & 0.0237 & 1.401 \\
\hline 24.69 & 36.45 & 530 & 618.9 & 0.0206 & 1.476 \\
\hline 26.69 & 41.22 & 530 & 629.9 & 0.0185 & 1.544 \\
\hline 28.69 & 46.1 & 530 & 639.6 & 0.017 & 1.607 \\
\hline
\end{tabular}

\section{A.2 ACM Mass Flow Rate}

Mass flow rate can be calculated using the air density and volumetric flow rate as follows.

$$
\dot{m}=\rho \dot{V} \quad(\text { Eqn. A.8) }
$$

Where $\rho$ is the air density, and $\dot{V}$ is the volumetric flow rate. The uncertainty is calculated in a similar way to the compressor efficiency uncertainty. First, the mass flow rate should be expressed in terms of measured quantities.

$$
\dot{m}=\left(\frac{R T}{P}\right) \dot{V} \quad(\text { Eqn. A.9) }
$$

The resulting uncertainty in mass flow rate for the following conditions is approximately 0.6 $\mathrm{lbm} / \mathrm{min}$, or $6.7 \%$.

Table A.3 Conditions for evaluating uncertainty in mass flow rate.

\begin{tabular}{|c|c|}
\hline Compressor Inlet Condition & Value \\
\hline Temperature [Deg. R] & 560 \\
\hline Pressure [psia] & 25 \\
\hline Volumetric Flow Rate [CFM] & 75 \\
\hline
\end{tabular}




\section{A.3 Compressor Power}

Compressor power can be calculated by the following equation:

$$
\dot{W}_{c}=\dot{m} c_{p}\left(T_{2 c}-T_{1 c}\right) \quad(\text { Eqn. A.10) }
$$

Using units of pound-mass, BTU/lbm/ ${ }^{\circ} \mathrm{R}$, and ${ }^{\circ} \mathrm{R}$, the result can be converted to hp by applying a conversion factor of 0.02358 to the right-hand side of Eqn. A.10.

The result of the uncertainty analysis for the following conditions is approximately $0.21 \mathrm{hp}$, or $7.5 \%$.

Table A.4 Conditions for evaluating uncertainty in ACM compressor power.

\begin{tabular}{|c|c|}
\hline Compressor Inlet and Outlet Conditions & Value \\
\hline Specific Heat, Constant Pressure [BTU/lbm/R] & 0.241 \\
\hline Inlet Temperature [Deg. R] & 530 \\
\hline Outlet Temperature [Deg. R] & 575 \\
\hline Inlet Pressure [psia] & 22.5 \\
\hline Volumetric Flow Rate [CFM] & 100 \\
\hline
\end{tabular}

\section{A.4 Turbine Power}

Turbine power can be expressed using the following equation.

$$
\dot{W}_{t}=\dot{m} c_{p}\left(T_{1 t}-T_{2 t}\right) \quad(\text { Eqn. A.11) }
$$

Alternatively, it can be expressed in terms of measured quantities.

$$
\dot{W}_{t}=\left(\frac{R T_{1 c}}{P_{1 c}}\right) \dot{V} c_{p}\left(T_{1 t}-T_{2 t}\right) \quad(\text { Eqn. A.12) }
$$

The resulting uncertainty is $0.16 \mathrm{hp}$, or $4.5 \%$.

\section{A.5 Cooling Capacity}

The cooling capacity can be expressed using the following equation. 


$$
\dot{Q}=\dot{m} c_{p}\left(T_{\text {ambient }}-T_{2 t}\right) \quad(\text { Eqn. A.13) }
$$

The cooling capacity can be expressed in terms of measured quantities.

$$
\dot{Q}=\left(\frac{R T_{1 c}}{P_{1 c}}\right) \dot{V} c_{p}\left(T_{1 t}-T_{2 t}\right) \quad(\text { Eqn. A.14) }
$$

The resulting uncertainty is $11.7 \mathrm{BTU} / \mathrm{min}$, or $6.1 \%$.

\section{A.6 Turbine Efficiency}

The turbine efficiency can be expressed as

$$
\eta=\frac{\dot{m} c_{p}\left(T_{1 t}-T_{2 t}\right)}{\dot{m}_{t} c_{p_{t}} T_{1 T}\left[\frac{P \gamma_{t}{ }^{-1} / \gamma_{t-1}}{{ }^{\gamma_{t}-1} / \gamma_{t}}\right]} \quad \text { (Eqn. A.15) }
$$

Alternatively, it can be expressed in terms of measured quantities.

$$
\eta=\frac{\left(T_{1 t}-T_{2 t}\right)}{T_{1 T}\left[\frac{P_{1 t} / P_{2 t}{ }^{-1} / \gamma_{t}}{P_{1 t} / P_{2 t}{ }^{-1} / \gamma_{t}}\right]} \quad \text { (Eqn. A.16) }
$$

The resulting uncertainty is $0.02[-]$.

\section{A.7 Intercooler Effectiveness}

The intercooler effectiveness can be expressed as

$$
E_{\text {htxr }}=\frac{\left(T_{1}-T_{2}\right)}{\left(T_{1}-T_{\text {ambient }}\right)} \quad(\text { Eqn. A.17) }
$$

The resulting uncertainty is $0.04[-]$. 


\section{A.8 Intercooler Pressure Drop}

The intercooler effectiveness can be expressed as

$$
\Delta P_{h t x r}=\left(P_{1}-P_{2}\right) \quad(\text { Eqn. A.18) }
$$

The resulting uncertainty is $0.03[-]$.

\section{A.9 Numerical Uncertainty Propagation Analysis}

Numerical uncertainty analysis was required for the dry-air rated parameters and relative humidity calculations. This was required because properties of moist air were found using the psychometric lookup functions in Engineering Equation Solver (EES), and an analytical solution for finding uncertainty was not possible. The calculated values that required numerical treatment are DAR COP, DAR Cooling Capacity, and the calculation of the relative humidity from wet-bulb, dry-bulb temperature, and ambient pressure measurements. More information about the method used in EES can be found at:

http://www.fchart.com/download/ees manual.exe

Alternatively, it can be found in PDF format at:

http://www.unh.edu/p2/ihf/ees.pdf 
Appendix B - Derivation of Equations for Air-cycle Analysis

B.1 Isentropic Compressor Power Requirement

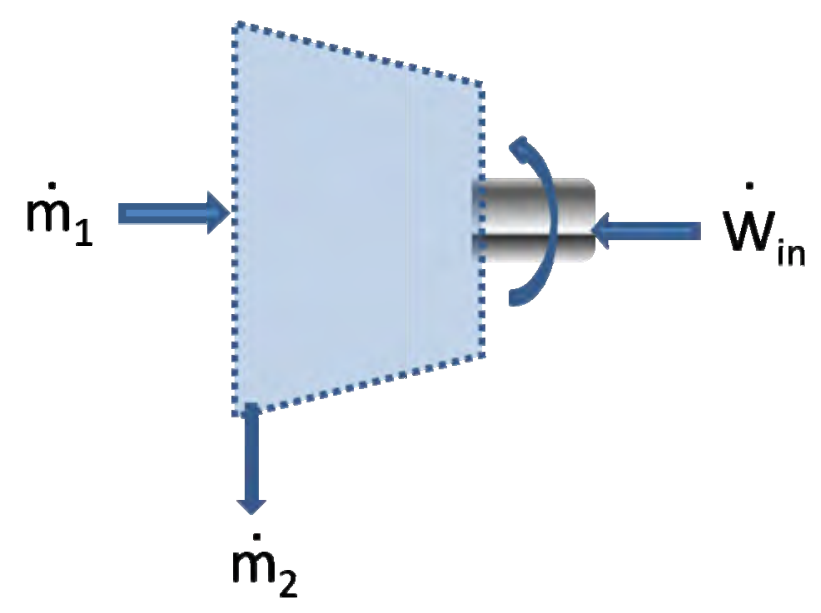

Figure B.1 Compressor control volume analysis.

\section{Assumptions}

- Isentropic

o Adiabatic

o Reversible

- Perfect Gas

o Ideal gas behavior

o Constant properties

Governing Equations

$$
\begin{gathered}
P v=R T \quad(\text { Eqn. B.1) } \\
P v^{\gamma}=\text { constant } \quad(\text { Eqn. B.2) }
\end{gathered}
$$




\section{Continuity}

$$
\dot{m}_{1}=\dot{m}_{2} \quad \text { (Eqn. B.3) }
$$

\section{Energy}

$$
\dot{W}_{\text {in }}=\dot{m}\left(h_{2}-h_{1}\right) \quad \text { (Eqn. B.4) }
$$

For a perfect gas, enthalpy can be found with the following equation

$$
h=c_{p}\left(T-T_{r e f}\right) \quad(\text { Eqn. B.5) }
$$

Substituting Eqn. B.5 into Eqn. B.4 results in the following energy equation

$$
\dot{W}_{i n}=\dot{m} c_{p}\left(T_{2}-T_{1}\right) \quad(\text { Eqn. B.6) }
$$

Using the equation of state for an ideal gas (Eqn. 3.1.1), we get

$$
\dot{W}_{i n}=\dot{m} \frac{c_{p}}{R}\left(P_{2} v_{2}-P_{1} v_{1}\right) \quad \text { (Eqn. B.7) }
$$

Rearranging Eqn. B.7 results in

$$
\dot{W}_{\text {in }}=\dot{m} \frac{c_{p}}{R} P_{2} v_{1}\left[\frac{v_{2}}{v_{1}}-\frac{P_{1}}{P_{2}}\right] \quad \text { (Eqn. B.8) }
$$

Applying the polytropic equation for an adiabatic boundary condition to Eqn. B.8 gives us

$$
\dot{W}_{\text {in }}=\dot{m} \frac{c_{p}}{R} P_{2} v_{1}\left[{\frac{P_{1}}{P_{2}}}^{1 / \gamma}-\frac{P_{1}}{P_{2}}\right] \quad \text { (Eqn. B.9) }
$$

Rearranging and defining pressure ratio as $P R_{C} \stackrel{\text { def }}{=} P_{2 C} / P_{1 C}$ we get,

$$
\dot{W}_{\text {in }}=\dot{m} c_{p} P_{1} v_{1}\left[P R_{C}{ }^{\gamma-1} / \gamma-1\right] \text { (Eqn. B.10) }
$$


Finally, substituting the ideal gas equation of state, we get

$$
\dot{W}_{C}=\dot{m} c_{p} T_{1 C}\left[P R_{C}{ }^{\gamma-1} / \gamma-1\right] \quad(\text { Eqn. B.11) }
$$

If we define an isentropic efficiency as

$$
\eta \stackrel{\text { def }}{=} \frac{\dot{W}_{\text {isentropic }}}{\dot{W}_{\text {actual }}} \quad \text { (Eqn. B.12) }
$$

Eqn. B.11 becomes

$$
\dot{W}_{C}=\dot{m} c_{p} \frac{T_{1}}{\eta_{c}}\left[P R_{C}{ }^{\gamma-1 / \gamma}-1\right] \quad(\text { Eqn. B.13) }
$$




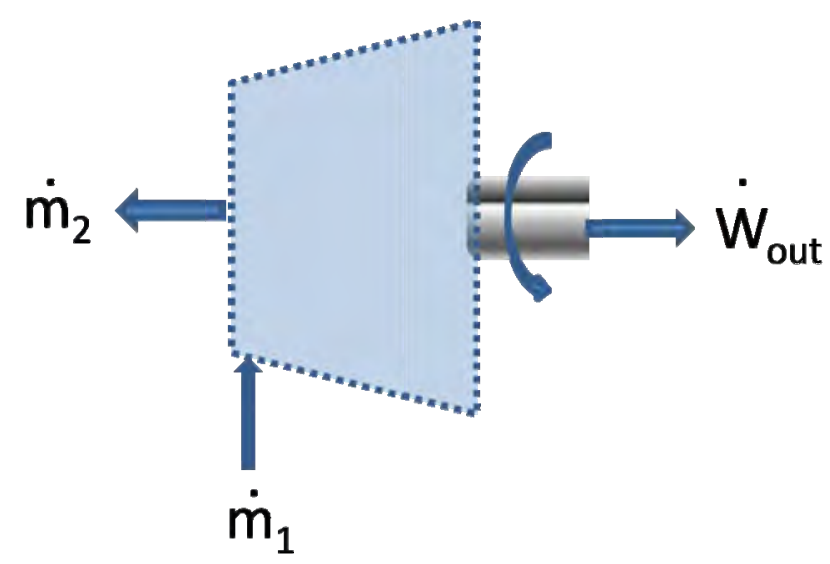

Figure B.2 Turbine control volume analysis.

\section{Assumptions}

- Isentropic

o Adiabatic

o Reversible

- Perfect Gas

o Ideal gas behavior

o Constant properties

\section{Governing Equations}

$$
\begin{gathered}
P v=R T \quad(\text { Eqn. B.14) } \\
P v^{\gamma}=\text { constant } \quad(\text { Eqn. B.15) }
\end{gathered}
$$

\section{Continuity}

$$
\dot{m}_{1}=\dot{m}_{2} \quad \text { (Eqn. B.16) }
$$




\section{Energy}

$$
\dot{W}_{\text {out }}=\dot{m}\left(h_{1}-h_{2}\right) \quad(\text { Eqn. B.17) }
$$

For a perfect gas, enthalpy can be found with the following equation

$$
h=c_{p}\left(T-T_{r e f}\right) \quad(\text { Eqn. B.18) }
$$

Substituting Eqn. B.18 into Eqn. B.17 results in the following energy equation

$$
\dot{W}_{\text {out }}=\dot{m} c_{p}\left(T_{1}-T_{2}\right) \quad \text { (Eqn. B.19) }
$$

Using the equation of state for an ideal gas (Eqn. B.14), we get

$$
\dot{W}_{\text {out }}=\dot{m} \frac{c_{p}}{R}\left(P_{1} v_{1}-P_{2} v_{2}\right) \quad(\text { Eqn. B.20) }
$$

Rearranging Eqn. B.20 results in

$$
\dot{W}_{\text {out }}=\dot{m} \frac{c_{p}}{R} P_{2} v_{1}\left[\frac{P_{1}}{P_{2}}-\frac{v_{2}}{v_{1}}\right] \quad(\text { Eqn. B.21) }
$$

Applying the polytropic equation for an adiabatic boundary condition to Eqn. B.21 gives us

$$
\dot{W}_{\text {out }}=\dot{m} \frac{c_{p}}{R} P_{2} v_{1}\left[\frac{P_{1}}{P_{2}}-\frac{P_{1}}{P_{2}}{ }^{1 / \gamma}\right]
$$

Rearranging and defining pressure ratio as $P R_{C} \stackrel{\text { def }}{=} P_{2 C} / P_{1 C}$ we get,

$$
\dot{W}_{\text {out }}=\dot{m} c_{p} P_{1} v_{1}\left[1-P R_{T}^{\gamma-1 / \gamma}\right] \quad \text { (Eqn. B.23) }
$$

Making a substitution with the ideal gas equation of state,

$$
\dot{W}_{\text {out }}=\dot{m} c_{p} T_{1}\left[1-P R_{T}^{\gamma-1 / \gamma}\right] \quad \text { (Eqn. B.24) }
$$


Dividing Eqn. B.24 by $P R_{C}{ }^{\gamma-1} / \gamma$ results in an equation of similar form to the compressor power equation. It is not necessary, but it is a more common form and makes it easier to remember for many people.

$$
\dot{W}_{\text {out }}=\dot{m} c_{p} T_{1}\left[\frac{P R_{T}^{\gamma-1 / \gamma}-1}{P R_{T}^{\gamma-1 / \gamma}}\right] \quad(\text { Eqn. B.25) }
$$

If we define an isentropic efficiency as

$$
\eta \stackrel{\text { def }}{=} \frac{\dot{W}_{\text {actual }}}{\dot{W}_{\text {isentropic }}} \quad \text { (Eqn. B.26) }
$$

Eqn. B. 25 becomes

$$
\dot{W}_{\text {out }}=\dot{m} c_{p} T_{1} \eta_{T}\left[\frac{P R_{T}^{\gamma-1} / \gamma_{-1}}{P R_{T}^{\gamma-1} / \gamma}\right] \quad \text { (Eqn. B.27) }
$$




\section{B.3 Bearing Losses}

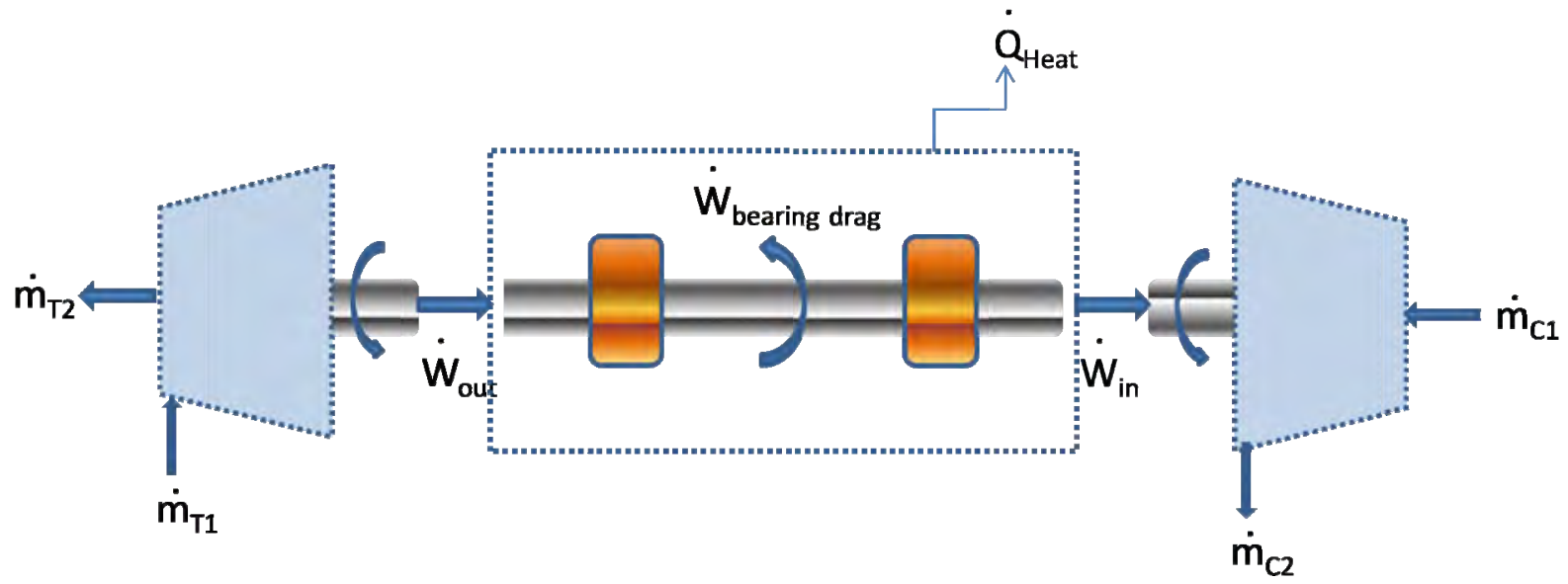

Figure B.3 Bearing system control volume analysis.

\section{Energy}

$$
\dot{W}_{\text {Bearing Drag }}=\dot{W}_{T}-\dot{W}_{C} \quad \text { (Eqn. B.28) }
$$

Substituting Eqn. B.27 and Eqn. B.13

$$
\dot{W}_{\text {Bearing Drag }}=\dot{m}_{c} c_{p_{c}} \frac{T_{1 C}}{\eta_{c}}\left[P R_{C}{ }^{\gamma_{c}-1} / \gamma_{c}-1\right]-\dot{m}_{t} c_{p_{t}} T_{1 T} \eta_{t}\left[\frac{P R_{t}{ }^{\gamma^{-1} / \gamma_{t-1}}}{P R_{t}{ }^{\gamma^{-1} / \gamma_{t}}}\right]
$$




\section{B.4 Isentropic Efficiency}

\section{B.4.1 Compressor Isentropic Efficiency Measurement}

Referring to Figure 3.1 for sensor layout, there are pressure and temperature measurements at the compressor inlet and outlet.

Starting with Eqn. B.12, and substituting Eqn. B.6 and Eqn. B.13

$$
\eta=\frac{\dot{m}_{c} c_{p_{c}} T_{1 C}\left[P R_{C}{ }^{\left.\gamma_{c}-1 / \gamma_{c-1}\right]}\right.}{\dot{m} c_{p}\left(T_{2_{\text {measured }}}-T_{1_{\text {measured }}}\right)} \quad(\text { Eqn. B.30) }
$$

This can be reduced to

$$
\eta_{c}=\frac{T_{1 C}\left[P R_{C}{ }^{\gamma_{c}-1} / \gamma_{c-1}\right]}{\left(T_{2 c}-T_{1 c}\right)}
$$

\section{B.4.2 Turbine Isentropic Efficiency Measurement with Bearing Losses Combined} Rearranging Eqn. B.28

$$
\dot{W}_{C}=\dot{W}_{T}-\dot{W}_{\text {Bearing Drag }} \quad \text { (Eqn. B.32) }
$$

In words, the next step is equating compressor power with effective turbine power. The righthand side is the lumped parameter containing the turbine power being reduced by the bearing losses. This reduces the apparent turbine isentropic efficiency. Turbine isentropic efficiency can be defined as

$$
\eta \stackrel{\text { def }}{=} \frac{\dot{W}_{\text {actual }}}{\dot{W}_{\text {isentropic }}}, \quad \text { (Eqn. B.33) }
$$

where the actual work represents the power consumed by the compressor, not the power produced directly by the turbine. This is typically the efficiency presented commercial turbocharger datasheets. 
Substituting Eqn. B.13 and Eqn. B.27 into Eqn. B.33

$$
\eta=\frac{\dot{m}_{c} c_{p_{c}} \frac{T_{1 C}}{\eta_{c}}\left[P R_{C}{ }^{\gamma_{c}-1} / \gamma_{c-1}\right]}{\dot{m}_{t} c_{p_{t}} T_{1 T} \eta_{t}\left[\frac{P R_{t}{ }^{-1} / \gamma_{t-1}}{\gamma_{t}-1 / \gamma_{t}}\right]}
$$

For most systems, the mass flow rate of the turbine is the mass flow rate of the compressor with the addition of a mass flow rate of fuel. For the $A C M$, the turbine mass flow rate is equal to the compressor mass flow rate, and, since the assumption of a perfect gas has been made, the gas properties remain constant throughout the cycle calculations. In this case, the turbine isentropic efficiency reduces to

$$
\eta=\frac{\frac{T_{1 C}}{\eta_{c}}\left[P R_{C}{ }^{\gamma-1 / \gamma}-1\right]}{T_{1 T}\left[\frac{P R_{t}{ }^{\gamma-1 / \gamma} / \gamma_{-1}}{P R_{t}{ }^{\gamma-1 / \gamma}}\right]}
$$

\section{B.4.3 Turbine Isentropic Efficiency Measurement without Bearing Losses Combined} Unlike the previous turbine isentropic efficiency calculations, the physical work will be represented as

$$
\dot{W}_{\text {actual }}=\dot{m}_{\text {measured }} c_{p}\left(T_{1, \text { measured }}-T_{2, \text { measured }}\right) \quad(\text { Eqn. B.36) }
$$

This is the physical power produced by an adiabatic turbine before bearing losses. Eqn. B.36 and Eqn. B.25 can be substituted into Eqn. B.33 to form

$$
\eta=\frac{\dot{m}_{\text {measured }} c_{p}\left(T_{1, \text { measured }}-T_{2, \text { measured }}\right)}{\dot{m}_{t} c_{p_{t}} T_{1 T}\left[\begin{array}{c}
\gamma_{t}{ }^{-1} / \gamma_{t-1} \\
\gamma_{t}-1 / \gamma_{t}
\end{array}\right]} \quad \text { (Eqn. B.37) }
$$

This can be reduced to 


$$
\eta=\frac{\left(T_{1, \text { measured }}-T_{2, \text { measured }}\right)}{T_{1 T}\left[\frac{P R_{t}{ }^{-1} / \gamma_{t-1}}{\gamma_{t}-1 / \gamma_{t}}\right]} \quad \text { (Eqn. B.38) }
$$




\section{B.5 Compressor Discharge Temperature}

Compressor discharge temperature can be calculated using the compressor work equation. In real cases, there will be a temperature rise due to isentropic, adiabatic compression and a component due to compressor inefficiency. The latter part is accounted for by the isentropic efficiency. Equating Eqn. B.6 and Eqn. B.13 results in the following equation.

$$
\dot{m} c_{p}\left(T_{2}-T_{1}\right)=\dot{m} c_{p} \frac{T_{1}}{\eta_{c}}\left[P R_{C}{ }^{\gamma-1 / \gamma}-1\right] \quad \text { (Eqn. B.39) }
$$

This results in an equation relating the compressor discharge temperature to a function of the compressor inlet temperature, pressure ratio, and compressor isentropic efficiency, three pieces of information that are typically known. By simplifying and rearranging Eqn. B.39, we get

$$
T_{2}=T_{1}+\frac{T_{1}}{\eta_{c}}\left[P R_{C}^{\gamma-1 / \gamma}-1\right] \quad(\text { Eqn. B.40) }
$$




\section{B.6 Corrected Compressor Flow Rate}

Corrected compressor flow rate maps the physical flow rate to a standard reference condition.

The compressor test conditions must be known to predict performance at conditions other than those identical to the testing conditions. Having one defined standard temperature and pressure condition for all compressor maps reduces chances of error by the engineer. Honeywell Turbo Technologies (Garrett Turbo) uses the compressor reference temperature and pressure as $545^{o_{R}}$ and $28.4 \mathrm{in} \mathrm{Hg}$, respectively [5].

$$
W_{C}^{*}=\frac{W_{C} \sqrt{T_{1 C} / 545}}{P_{1 C} / 28.4} \quad(\text { Eqn. B.41) }
$$

\section{B.7 Corrected Compressor Speed}

The corrected compressor speed needs to be corrected in a similar fashion to compressor flow rate, except for dependence on pressure [5].

$$
N_{C}^{*}=\frac{N_{C}}{\sqrt{T_{1 C / 545}}} \text { (Eqn. B.42) }
$$

\section{B.8 Turbine Discharge Temperature}

The turbine outlet temperature cannot be predicted exactly from typical turbine maps. Most turbine maps provide the turbine efficiency including bearing losses, so this will indicate less than the actual power the turbine is producing. This will tend to underestimate the temperature drop across the turbine. For most applications of turbochargers, the turbine discharge temperature is not of much concern; the power provided to the compressor is of more interest. 
Starting with a compressor-turbine power balance (Eqn. B.13 and Eqn. B.27), the turbine discharge temperature can be found.

$$
\dot{m} c_{p} \frac{T_{1 c}}{\eta_{c}}\left[P R_{C}^{\gamma-1 / \gamma}-1\right]=\dot{m} c_{p}\left(T_{1 T}-T_{2 T}\right) \quad \text { (Eqn. B.43) }
$$

The compressor and turbine flow rates are the same and, it is being modeled as a perfect gas, so it reduces to

$$
\frac{T_{1 c}}{\eta_{c}}\left[P R_{C}^{\gamma-1 / \gamma}-1\right]=\left(T_{1 T}-T_{2 T}\right) \quad(\text { Eqn. B.44) }
$$

This can be arranged in the form

$$
T_{2 T}=T_{1 T}-\frac{T_{1 c}}{\eta_{c}}\left[P R_{C}^{\gamma-1 / \gamma}-1\right] \quad(\text { Eqn. B.45) }
$$

\section{B.9 Corrected Turbine Flow Rate}

Corrected turbine flow rate calculation is the same as corrected to corrected compressor flow rate, except for reference conditions. Honeywell Turbo Technologies (Garrett Turbo) uses a turbine reference temperature and pressure of $519^{\circ}$ and $29.92 \mathrm{in} \mathrm{Hg}$, respectively [5].

$$
W_{T}^{*}=\frac{W_{T} \sqrt{T_{1 T} / 519}}{P_{1 T} / 29.92} \quad(\text { Eqn. B.46) }
$$




\section{B.10 Corrected Turbine Speed}

Corrected turbine speed calculation is the same as for corrected compressor speed, with the exception of reference temperature [5].

$$
N_{T}^{*}=\frac{N_{T}}{\sqrt{T_{1 T} / 519}} \text { (Eqn. B.47) }
$$

\section{B.11 Dry Air-Rated Temperature}

Treating systems with multi-component flow can be difficult and cumbersome. The dry airrated method allows the engineer to treat moist air systems as a single-component gas. The dry air rated temperature is a pseudo temperature of dry air that has equivalent enthalpy to the original air-entrained water mixture if the entrained water were to adiabatically vaporize. This can greatly simplify calculations by allowing treatment as an ideal gas or simplified further to a perfect gas.

\section{Appendix C - Compressor-Turbine Matching Process}

Before beginning the cycle analysis, compressor and turbine performance maps are needed from the manufacturer. These maps provide the isentropic efficiency of the components over their operating range. Obtaining a good turbine-compressor match is a critical step in achieving minimum turbine discharge temperatures. 


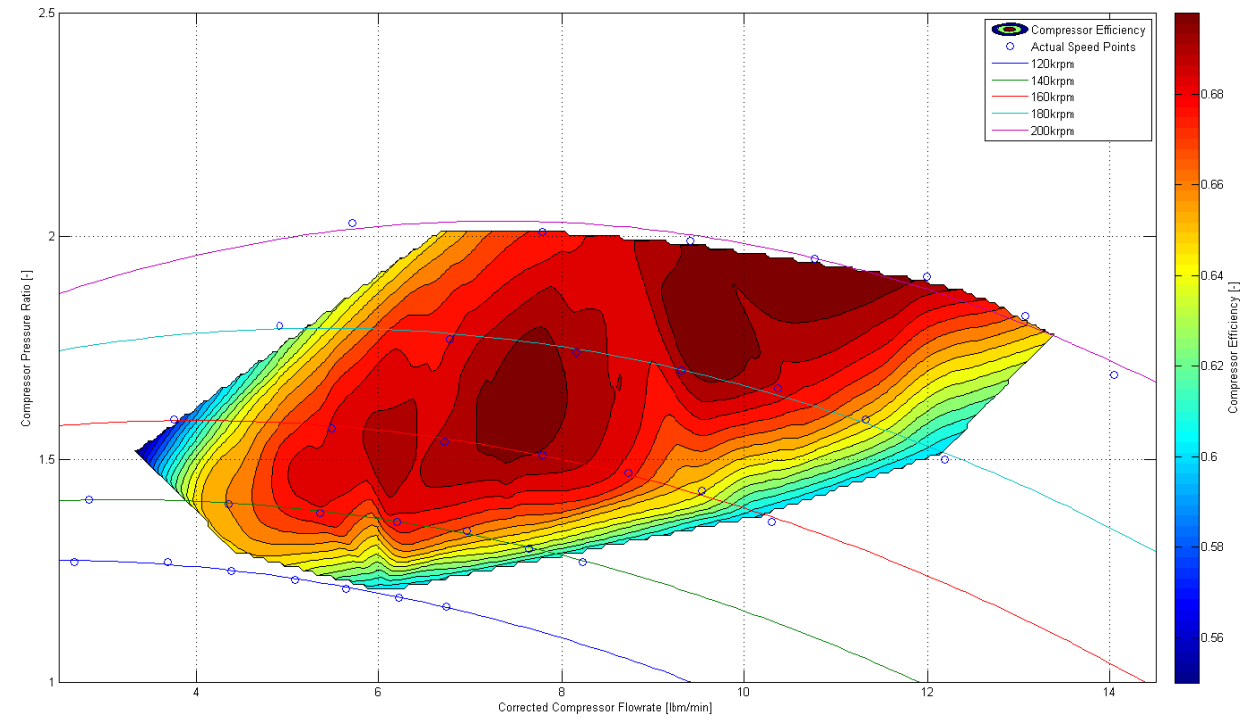

Figure C.1 GT1244 compressor performance map.

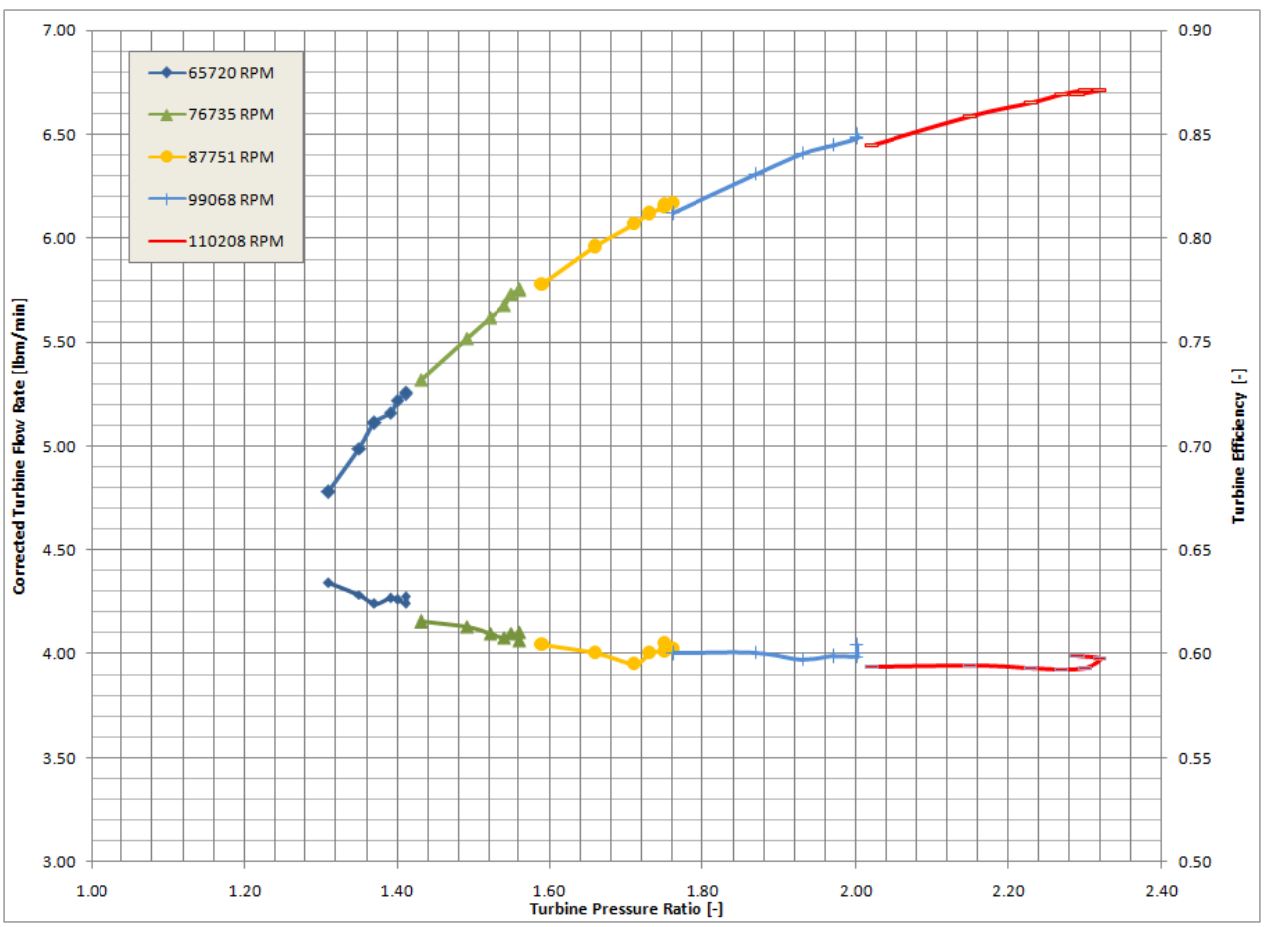

Figure C.2 GT1244 turbine performance map. 
To start the compressor-turbine analysis, it is important to start with the compressor first because the compressor performance map will define a corrected compressor speed based on the pressure ratio and corrected compressor flow rate. One can assume values for corrected compressor flow rate and pressure ratio. Using these assumed values the compressor map will provide compressor speed and isentropic efficiency. Using Eqn. C.1, the required compressor power can be calculated.

$$
\dot{W}_{C}=\dot{m} c_{p} \frac{T_{1}}{\eta_{c}}\left[P R_{C}{ }^{\gamma-1} / \gamma-1\right] \quad(\text { Eqn. C.1) }
$$

The next step is to convert corrected quantities to physical quantities, such as compressor mass flow rate and speed. These can be modified to the actual operating conditions by the following equations.

$$
\begin{gathered}
W_{c}=W_{c}^{*} \frac{\sqrt{T_{1 c / 545}}}{P_{1 c / 28.4}} \quad(\text { Eqn. C.2) } \\
N_{c}=N_{c}^{*} \sqrt{T_{1 c / 545}} \quad(\text { Eqn. C.3) }
\end{gathered}
$$

The compressor outlet temperature can be determined using the known or assumed compressor inlet temperature, compressor pressure ratio, and compressor efficiency.

$$
T_{2}=T_{1}+\frac{T_{1}}{\eta_{c}}\left[P R_{C}^{\gamma-1 / \gamma}-1\right] \text { (Eqn. C.4) }
$$

The next component after the compressor will be the heat exchanger, followed by the turbine. There will be a pressure drop across the heat exchanger. Typical values can be assumed for the pressure drop and effectiveness, unless actual heat exchanger data is available. In this project, engineering data on the heat exchanger was not available, so the pressure drop was assumed to 
be $1 p s i$ and effectiveness to be 0.80 . From this, turbine inlet pressure and temperature can be found.

$$
\begin{gathered}
P_{1 T}=P_{2 c}-\Delta P_{H T X R} \text { (Eqn. C.5) } \\
T_{1 T}=T_{2 c}-\varepsilon\left(T_{2 c}-T_{a m b}\right)(\text { Eqn. C.6) }
\end{gathered}
$$

Typically the turbine outlet pressure can be assumed to be the ambient pressure. The turbine pressure ratio can be found from the following equation.

$$
P R_{T}=P_{1 T} /_{P_{2 T}} \text { (Eqn. C.7) }
$$

To acquire data from the turbine performance map, corrected turbine mass flow rate and speed are required. These can be found from the following equations.

$$
\begin{gathered}
W_{T}^{*}=\frac{W_{T} \sqrt{T_{1 T} / 519}}{P_{1 T} / 29.92} \text { (Eqn. C.8) } \\
N_{T}^{*}=\frac{N_{T}}{\sqrt{T_{1 T / 519}}} \quad \text { (Eqn. C.9) }
\end{gathered}
$$

Turbine efficiency can be found from the turbine performance map at this point, and turbine power can be found using the following equation. The corrected turbine mass flow rate must fall on the flow lines on the turbine performance map, and if they do not, conservation of mass is not satisfied. Iteration with new assumed values is required.

$$
\dot{W}_{\text {out }}=\dot{m} c_{p} T_{1} \eta_{T}\left[\frac{P R_{T}{ }^{\gamma-1} / \gamma_{-1}}{P R_{T}^{\gamma-1} / \gamma}\right] \quad(\text { Eqn. C.10) }
$$

Once this is complete the turbine power generation can be compared to the compressor power consumption. They should be relatively close, within a tolerance determined by the engineer. If 
not, a new corrected compressor mass flow rate and pressure ratio can be selected and repeat this process until mass, power, and speed are balanced. 


\section{Appendix D - Detailed Calibration Procedure}

The experimental data is collected using a National Instruments DAQPad 6015 data acquisition (DAQ) system and a NI SCB-68 breakout box with cold-junction compensation (CJC). The type-T thermocouple inputs are configured as a differential input for noise cancellation purposes. Noise is an issue in the thermocouple readings because the span of the wire reached up to 15 feet. Shielding with single-ended grounding provides enough protection from line noise to obtain standard accuracy of $+/-1 C$ for type- $T$ thermocouples. Each thermocouple channel is calibrated using a Fluke 714 Thermocouple Calibrator unit. The pressure transducers are automotive 3-bar MAP (Manifold Absolute Pressure) sensors. The pressure transducers are powered from a $+5 \mathrm{~V}$ source available from the $\mathrm{NI}$ equipment, as to provide a stable input to improve the quality of the measurements. The pressure transducers are calibrated using a Fluke 718 Series Pressure Calibrator unit.

The laminar flow element (LFE) is a Meriam 50MC2-2. The calibration sheet that relates the volumetric flow rate to pressure differential correlation is provided by the manufacturer. The pressure differential across the LFE is measured by a Meriam 2110P Smart Pressure Gauge differential pressure transducer. The differential pressure transducer is calibrated using a Fluke 718 Series Pressure Calibrator unit. The two calibration curves, from the LFE and differential pressure transducer, are combined to relate the volumetric flow rate to a voltage output.

All measurements were oversampled and averaged to provide the most stable measurements. The noise measured was nearly normally distributed, so averaging does not generate a significant offset in the readings. In any case, the oversampling and averaging method used was employed at the time of calibration, so any offset is captured in the calibrations obtained. The largest components of the noise were at $60 \mathrm{~Hz}$ and $120 \mathrm{~Hz}$. These two frequencies correlate to 
noise that is generated by single-phase $A C$ and full-bridge rectified single-phase AC. The sensor calibration was checked periodically to ensure proper sensor operation. The severity of the electromagnetic interference (EMI) in the testing facility can be seen in the next plots.

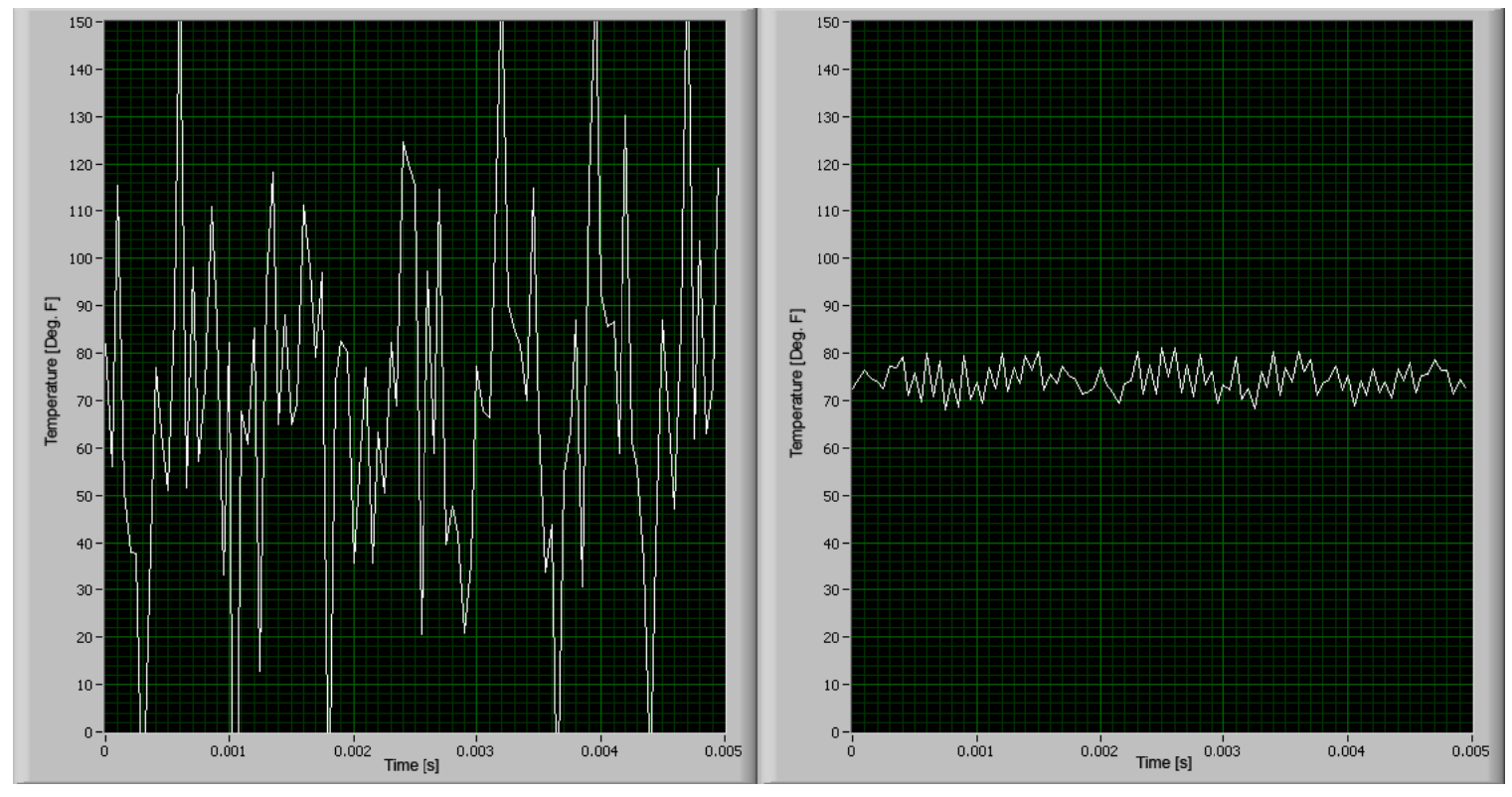

Figure D.1 Thermocouple measurement noise (pre-filtered) before and after shielding the wires.

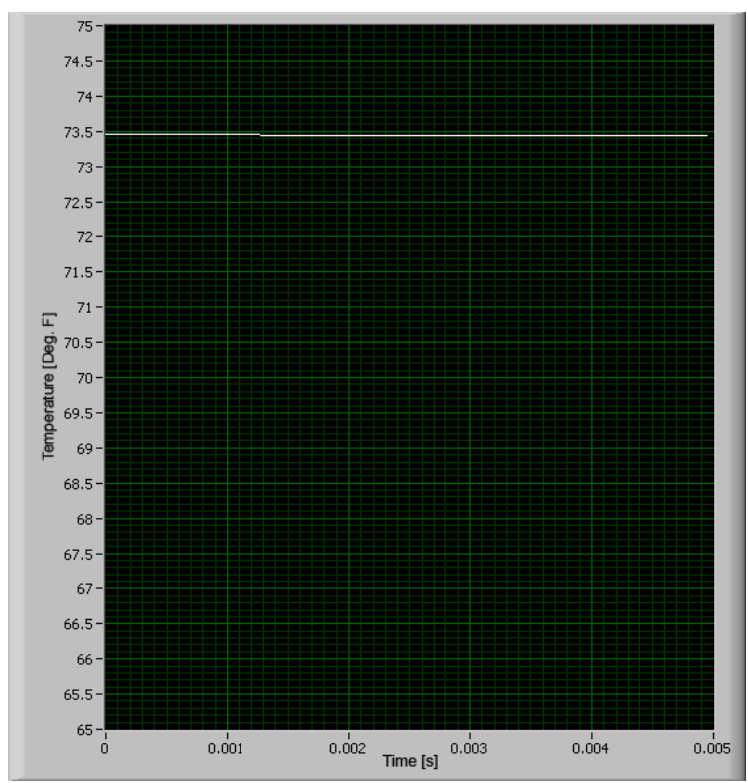

Figure D.2 Temperature measurement output after oversampling and averaging. 
The temperature output from the DAQ system always agreed with the Fluke calibrator unit within 1.7 Deg. F, after implementing the shielding, oversampling, and averaging. This is approximately the standard limit of error for the thermocouple wire used. 


\section{Appendix E - Sensor Calibration Charts}

\section{E.1 Pressure Transducer Calibration}
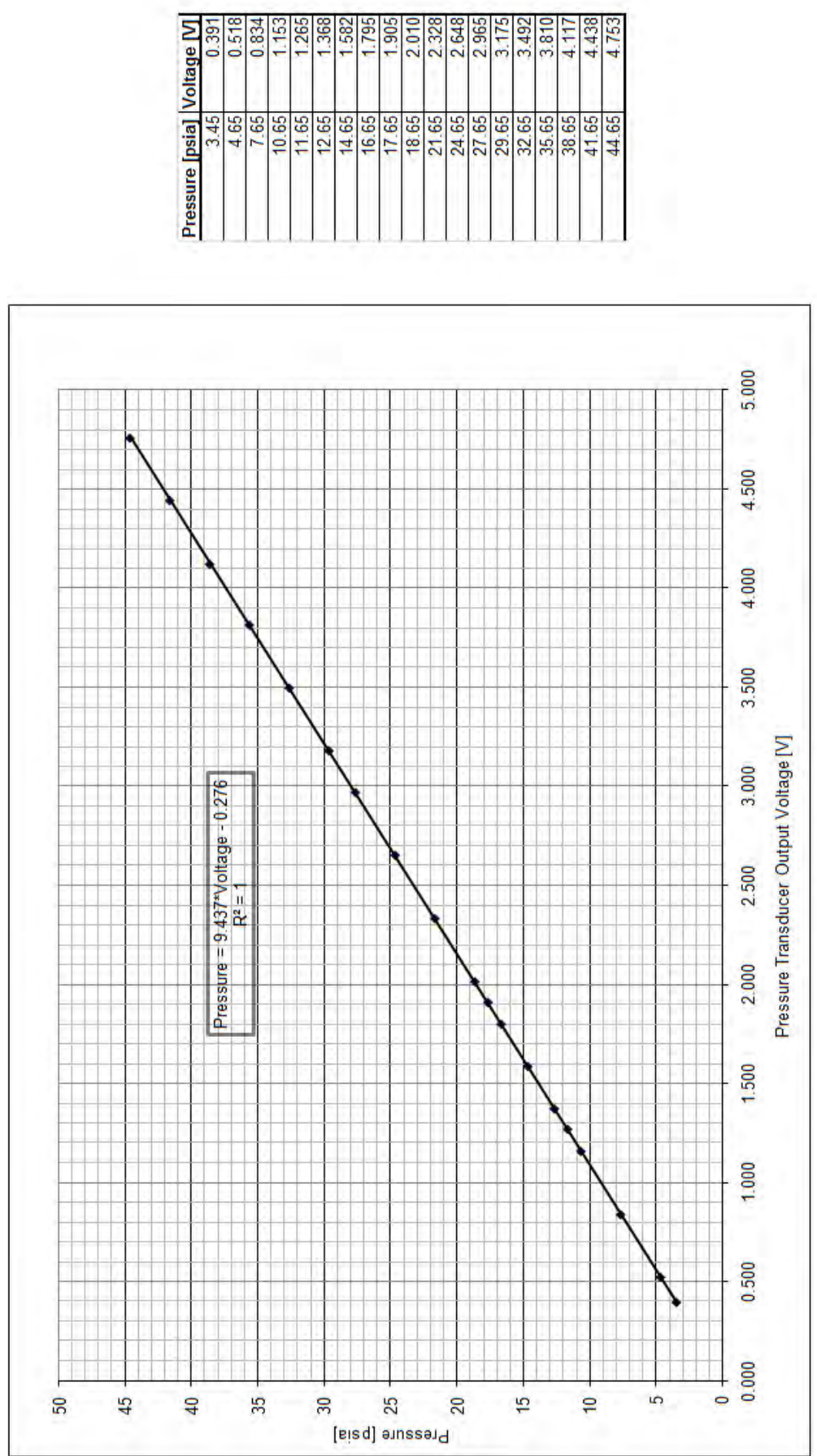

Figure E.1 MAP sensor calibration chart. 


\section{E.2 Laminar Flow Element and Differential Pressure Transducer Calibration}

The laminar flow element and differential pressure transducer setup requires multiple calibrations. The first calibration comes with the LFE manufacturer, and this relates volumetric flow rate to a pressure differential across the element. It is important to note that in order to make accurate measurements with a LFE, using the provided calibration sheet, the required straight, smooth pipe entrance and exit lengths must be at an approximate minimum of 10 and 5 pipe diameters, respectively.

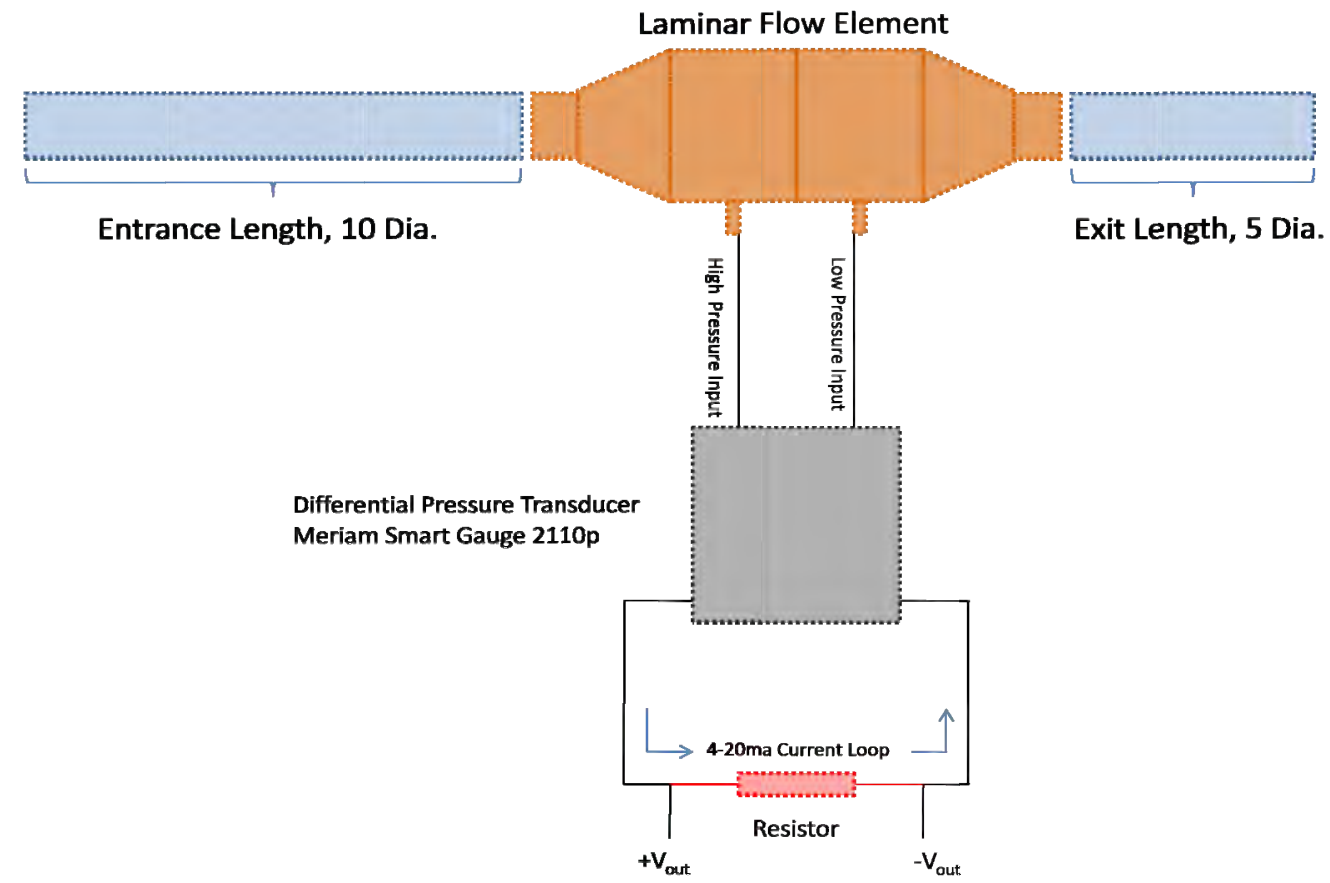

Figure E.2 This schematic shows the configuration of the LFE, differential pressure transducer, and resistor. 


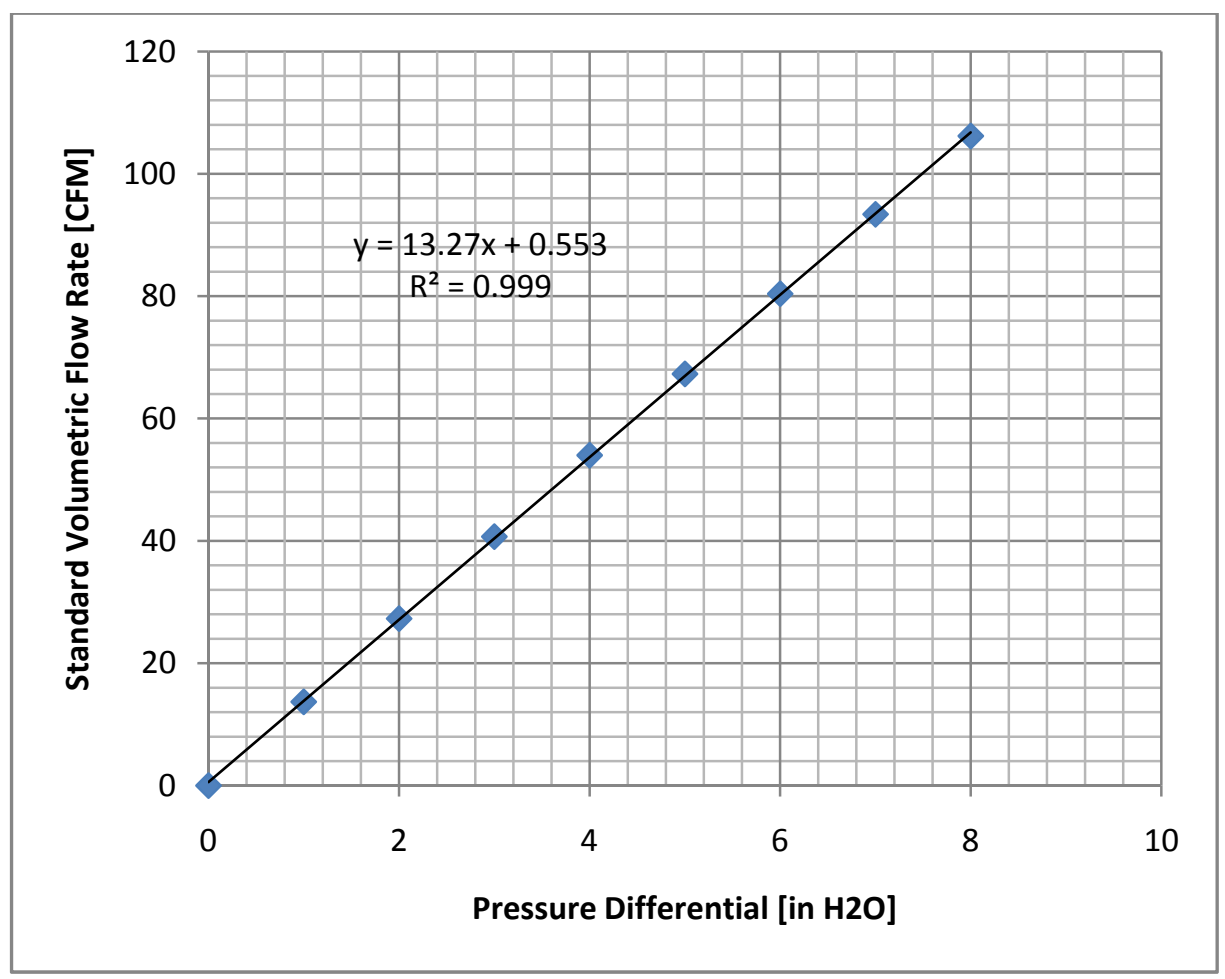

Figure E.3 Laminar flow element calibration curve. Data is provided by Meriam Process Technologies.

The second calibration required is for the differential pressure transducer to relate output voltage to the pressure differential. The sensor provides a standard 4-20ma current loop output. This is an excellent choice for transmitting a signal accurately over larger distances. While there is a voltage drop associated with a length of wire, there is continuity in the current through the wire. One drawback is that the current must be converted to a voltage by placing a resistor in the loop and measuring the voltage differential across the resistor. There is a tolerance on that resistor that is often $5-10 \%$. There are closer tolerances available at additional cost, but a pressure transducer calibration with the implemented resistor in place will provide the most accurate measurements. 


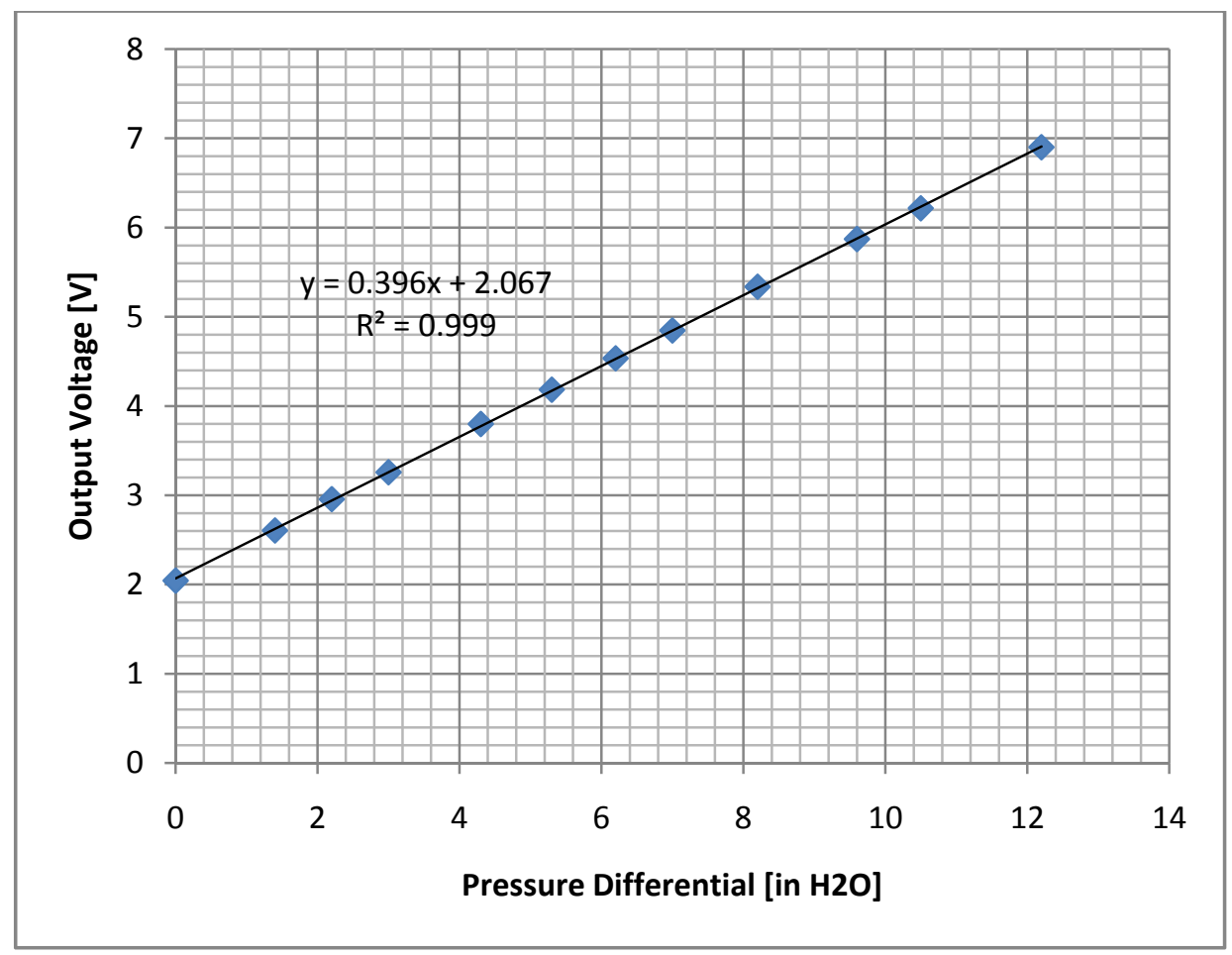

Figure E.4 Differential pressure transducer and resistor combined calibration.

Once both calibrations are complete, you have a relationship between volumetric flow rate and pressure drop and another relationship between pressure drop and voltage. The two can be related by doing a linear curve-fit on each calibration, and using the resulting equations to make a new calibration chart relating the voltage output to volumetric flow rate. 


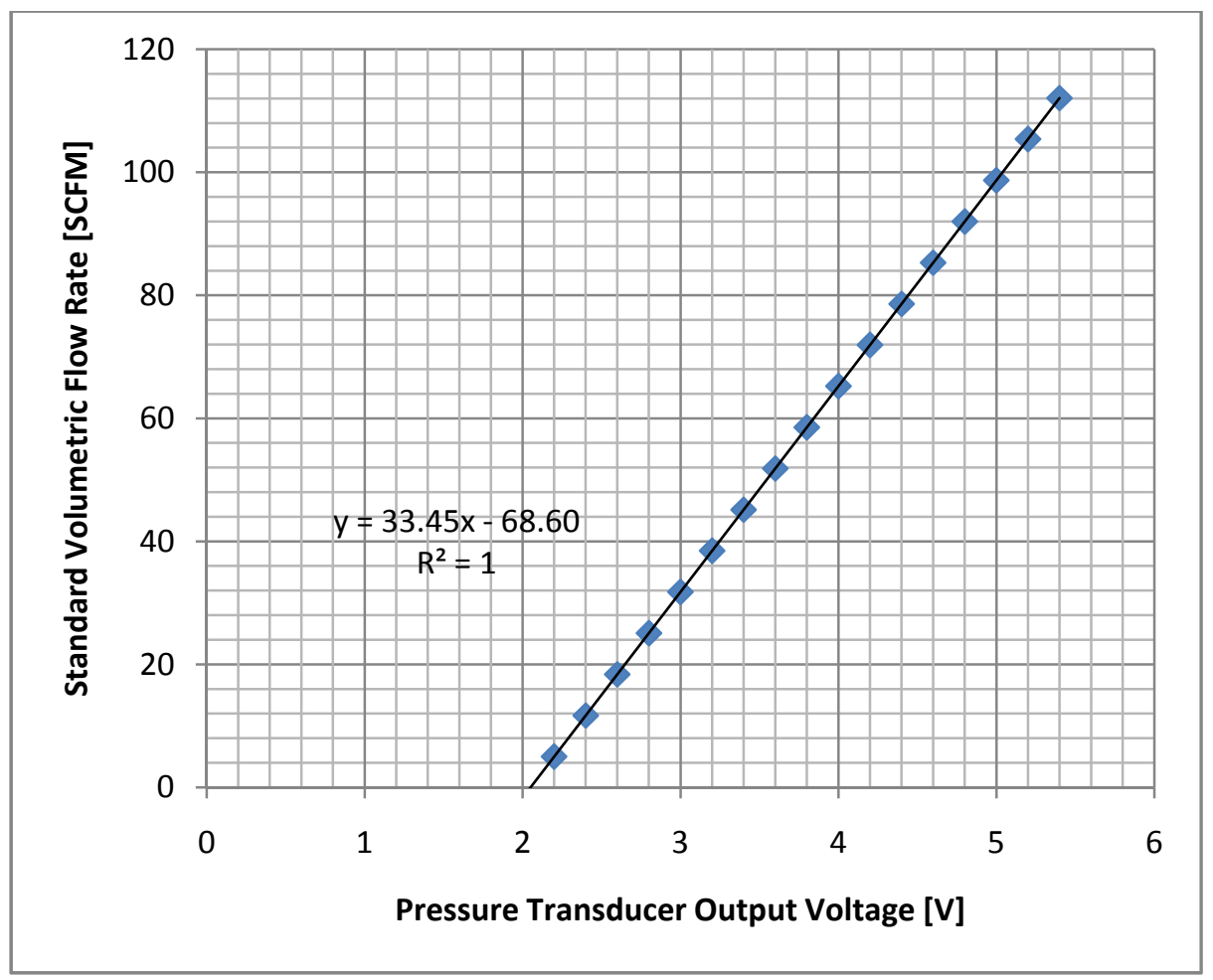

Figure E.5 Laminar flow element setup calibration.

\section{E.3 Thermocouple Calibration}

The thermocouples were calibrated using a Fluke 714 Calibrator Unit, as described in Appendix D. With sufficient electro-magnetic interference (EMI) protection, the standard type-T calibration, as implemented by default in Labview, was sufficient to match the calibration unit within 1.7 [Deg. F] over the expected temperature range of ACM operation. This range was expected to be approximately 32-180 [Deg. F]. 


\section{Appendix F - Perkins Diesel Performance, Stock Turbocharger}

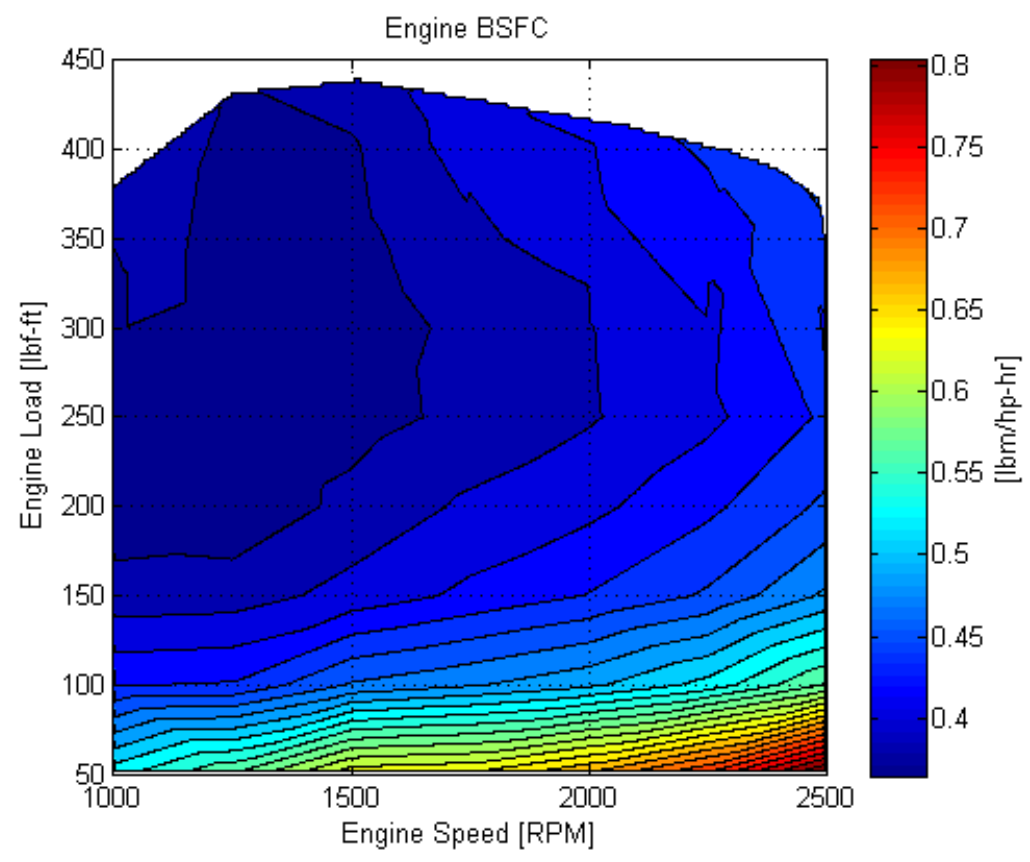

Figure F.1 BSFC contour map using the stock turbocharger.

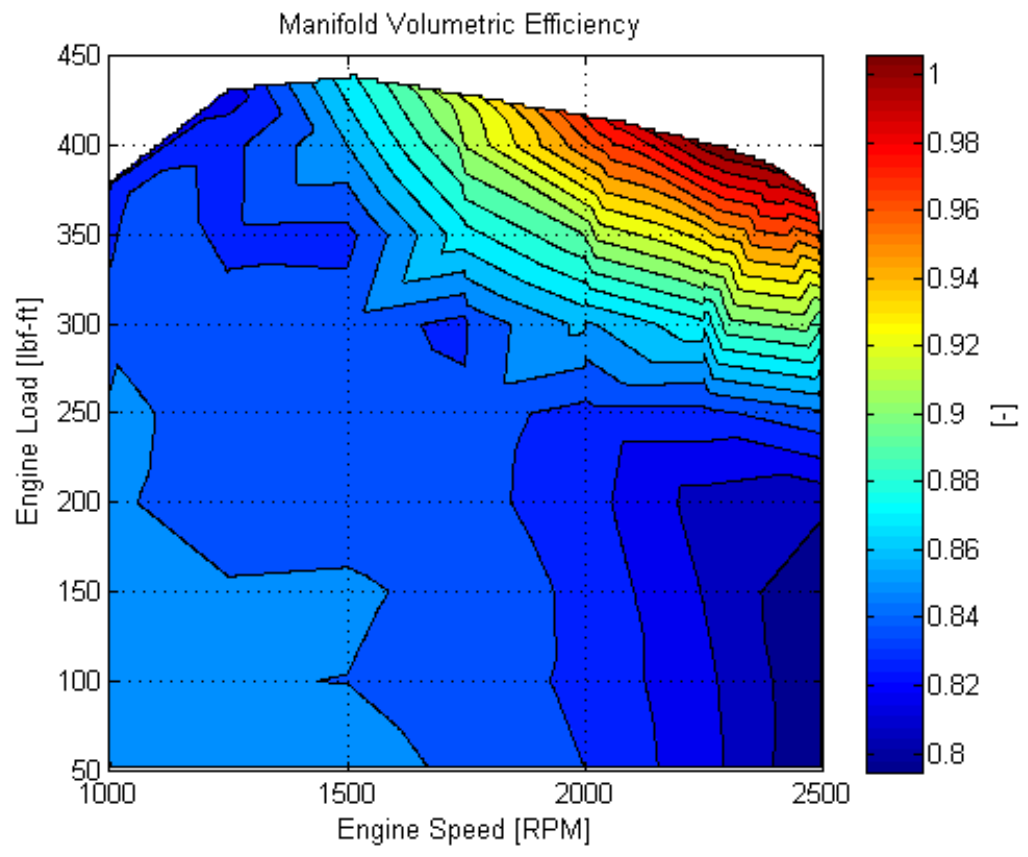

Figure F.2 VE contour map using the stock turbocharger. 


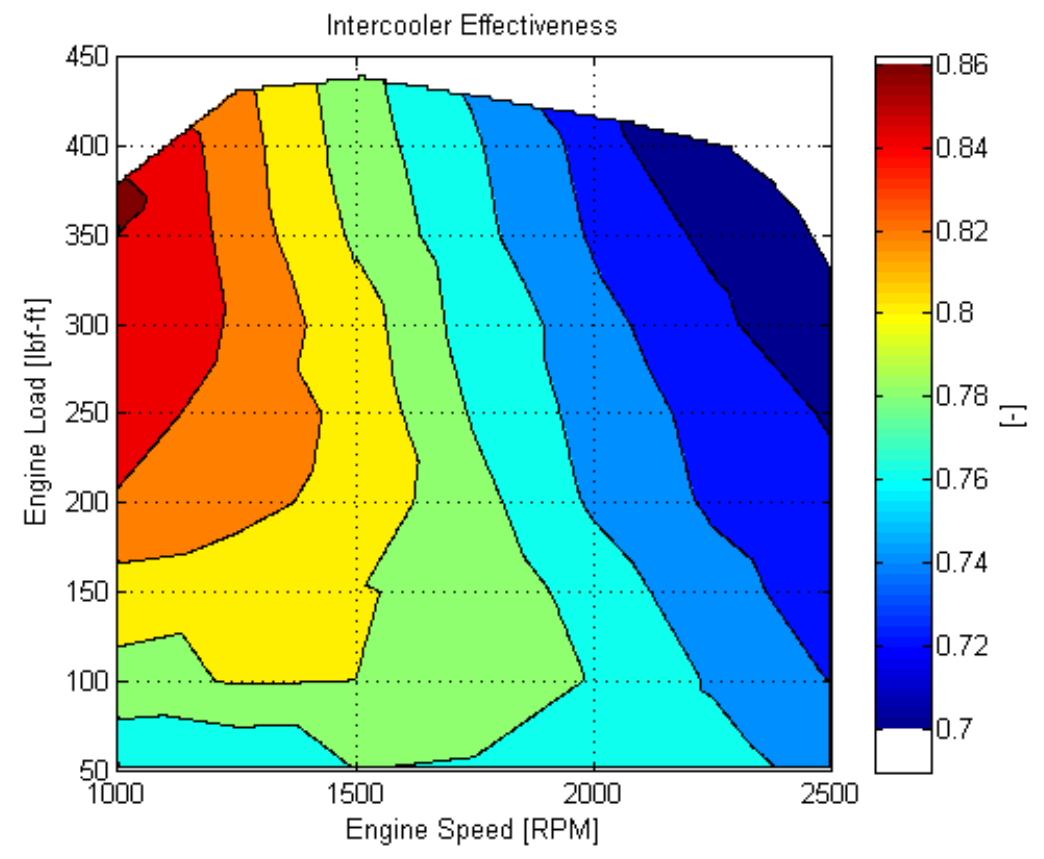

Figure F.3 Intercooler effectiveness contour map using the stock turbocharger.

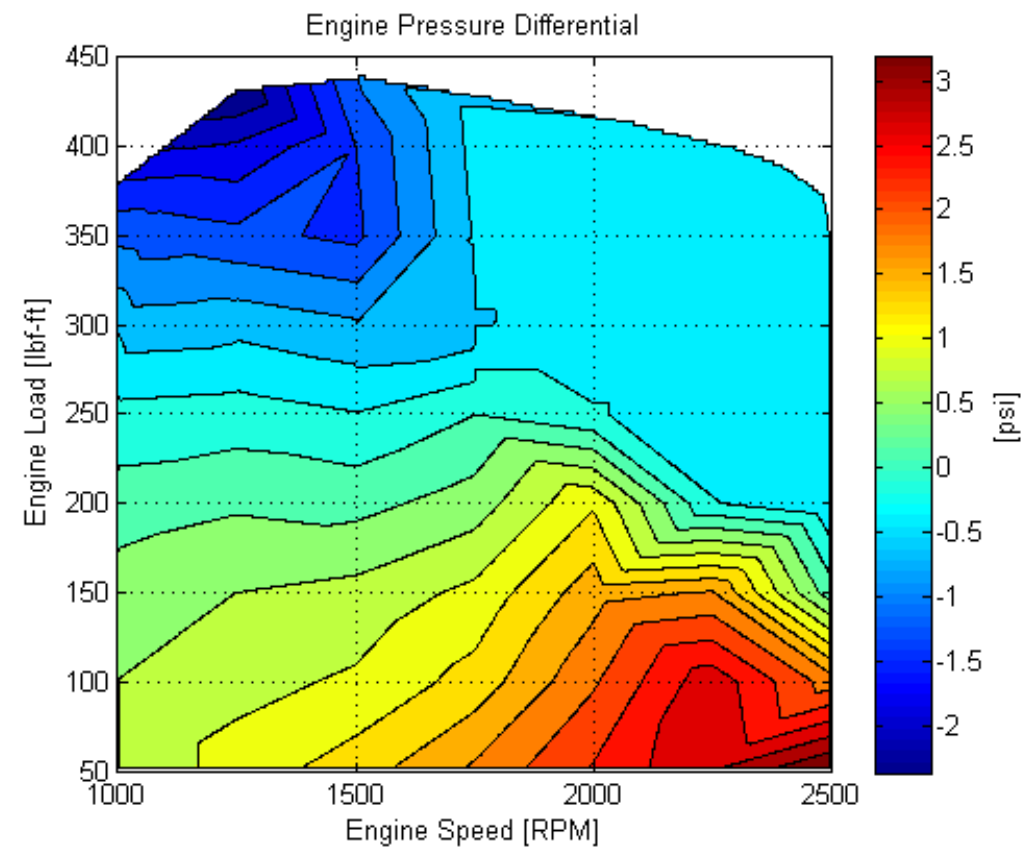

Figure F.4 Pressure differential between the exhaust and intake manifolds. (P_int - P_exh) 


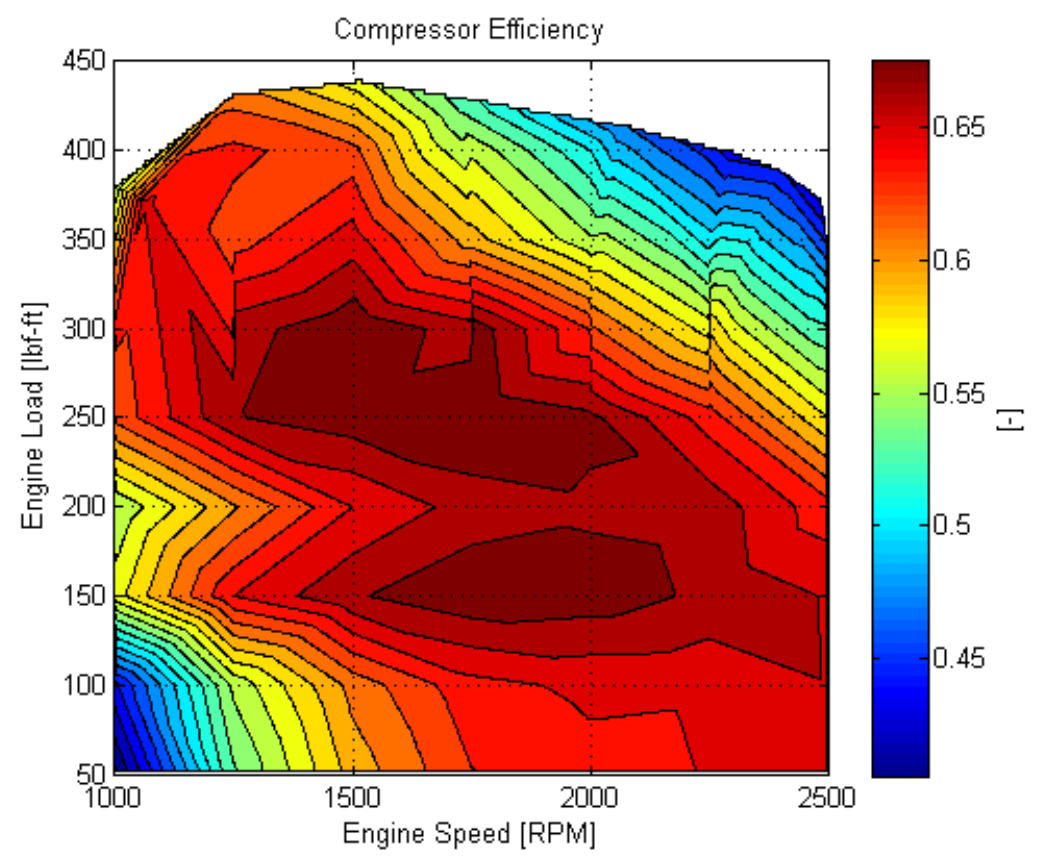

Figure F.5 Engine turbocharger compressor efficiency.

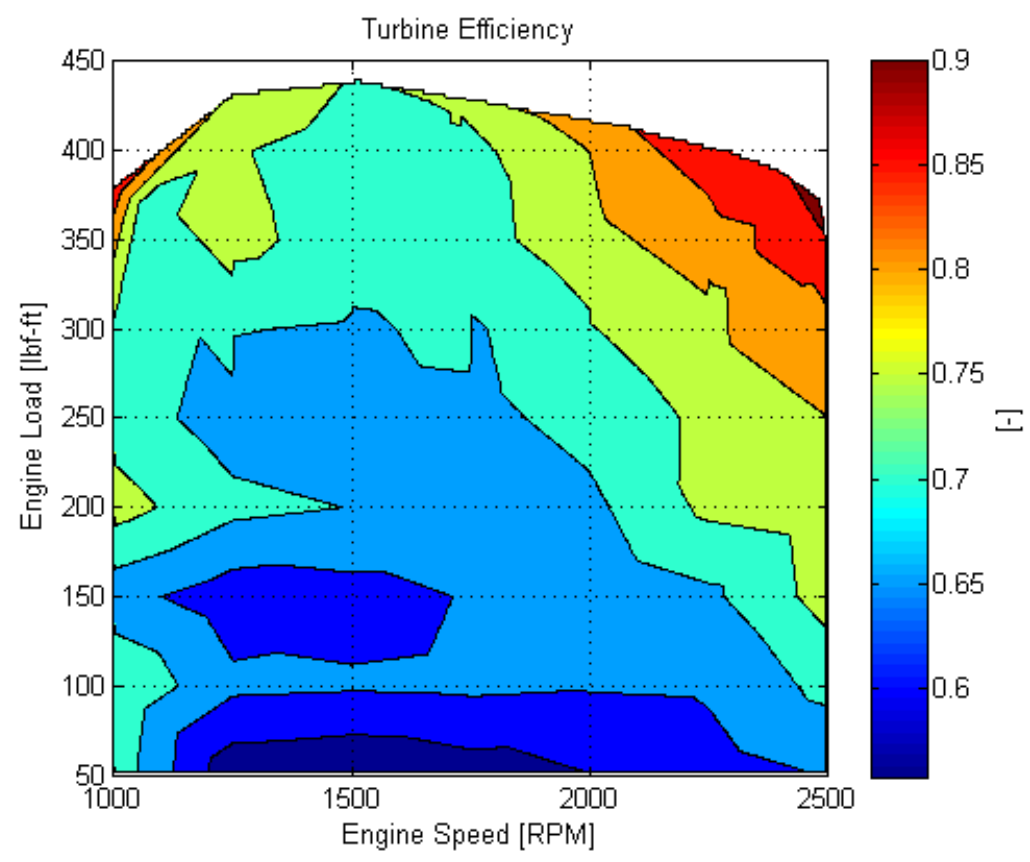

F.6 Engine turbocharger turbine efficiency. 


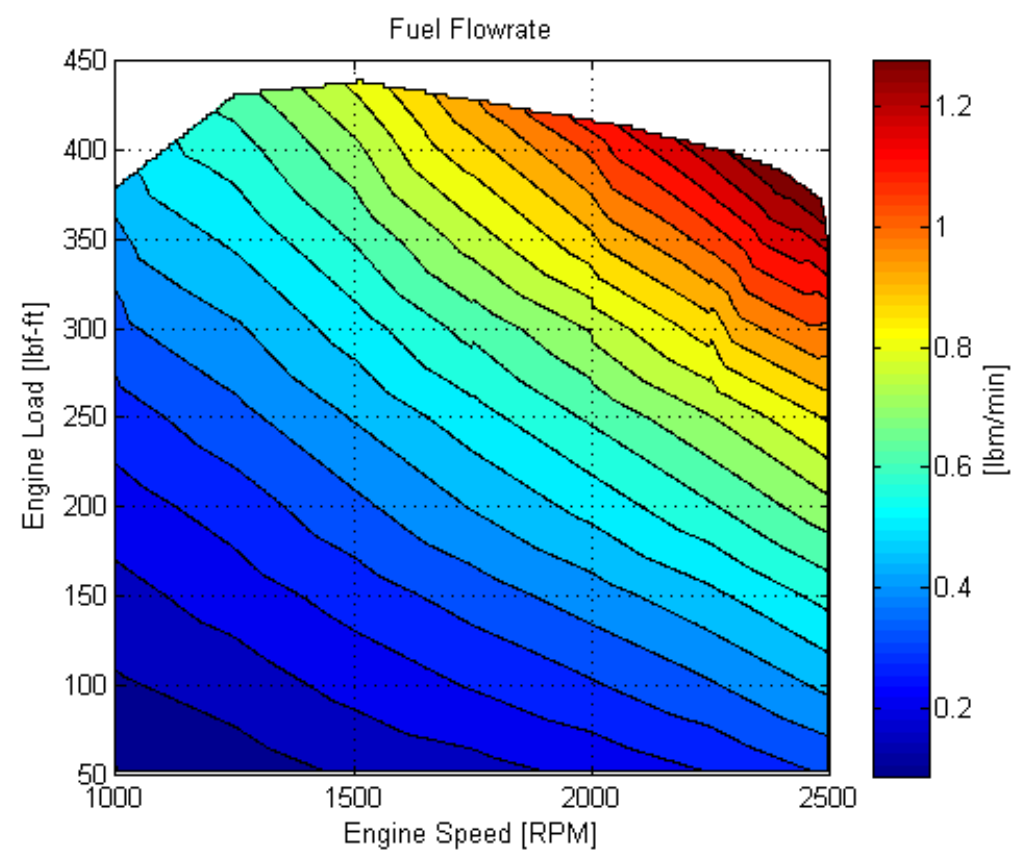

F.7 Engine fuel mass flow rate. 


\section{Appendix G - Perkins Diesel Performance, VNT Turbocharger}

The VNT turbocharger was tested over a smaller torque and speed range due to fuel measurement time limitations and the extra time necessary for turbine vane position adjustments.

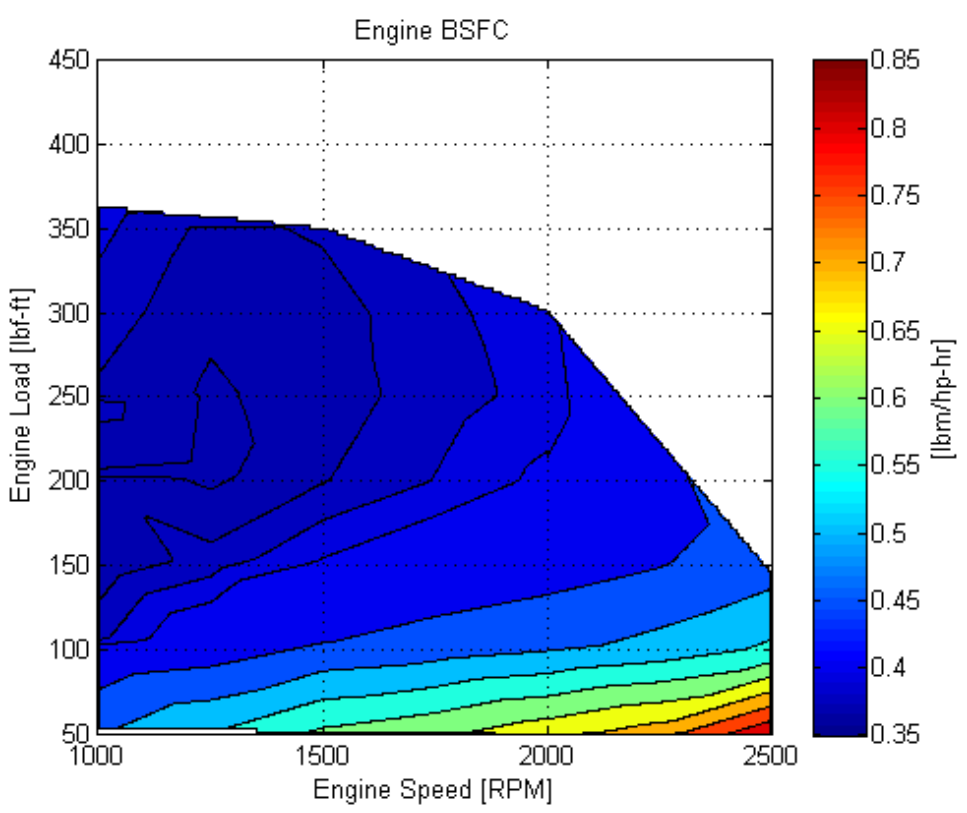

Figure G.1 BSFC contour map using the VNT turbocharger

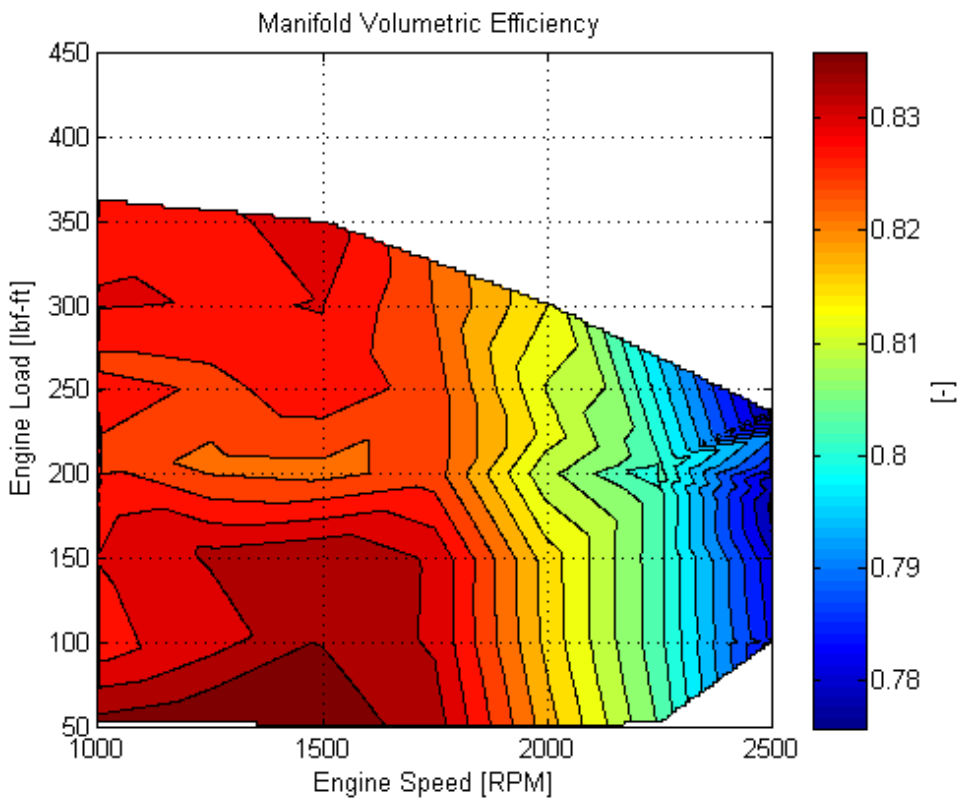

Figure G.2 VE contour map using the VNT turbocharger. 


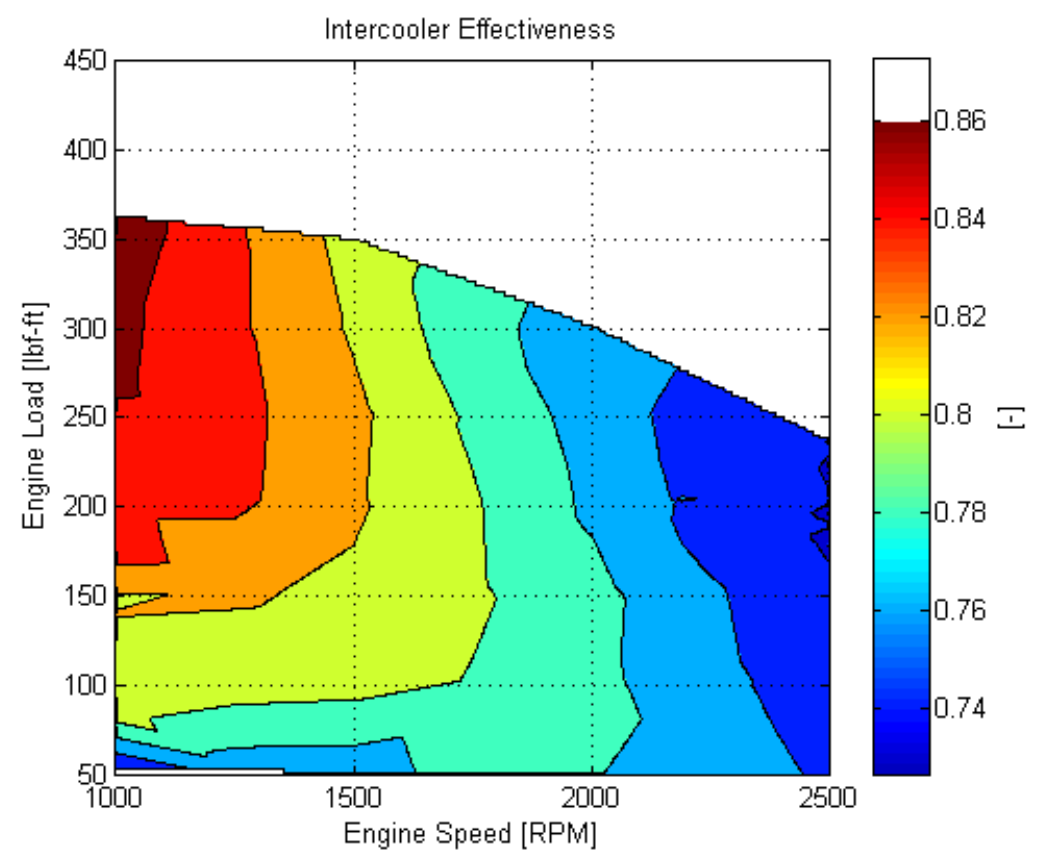

Figure G.3 Intercooler effectiveness contour map using the VNT turbocharger.

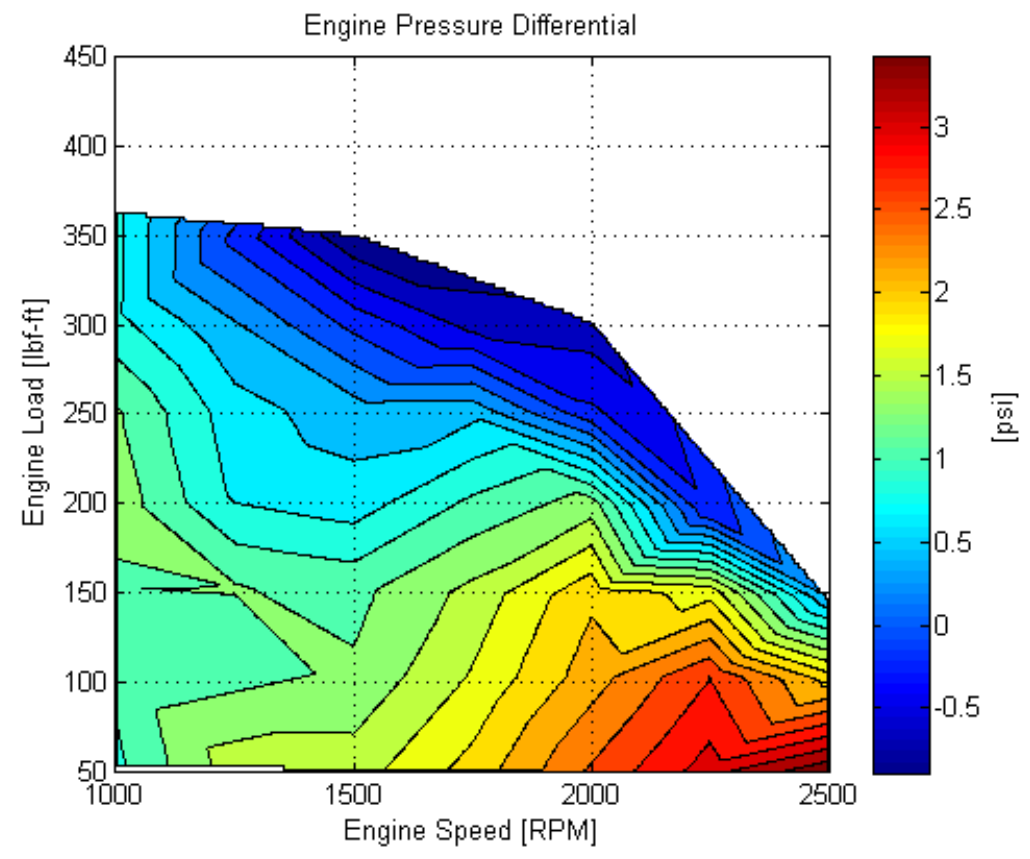

Figure G.4 differential between the exhaust and intake manifolds. (P_int - P_exh) 


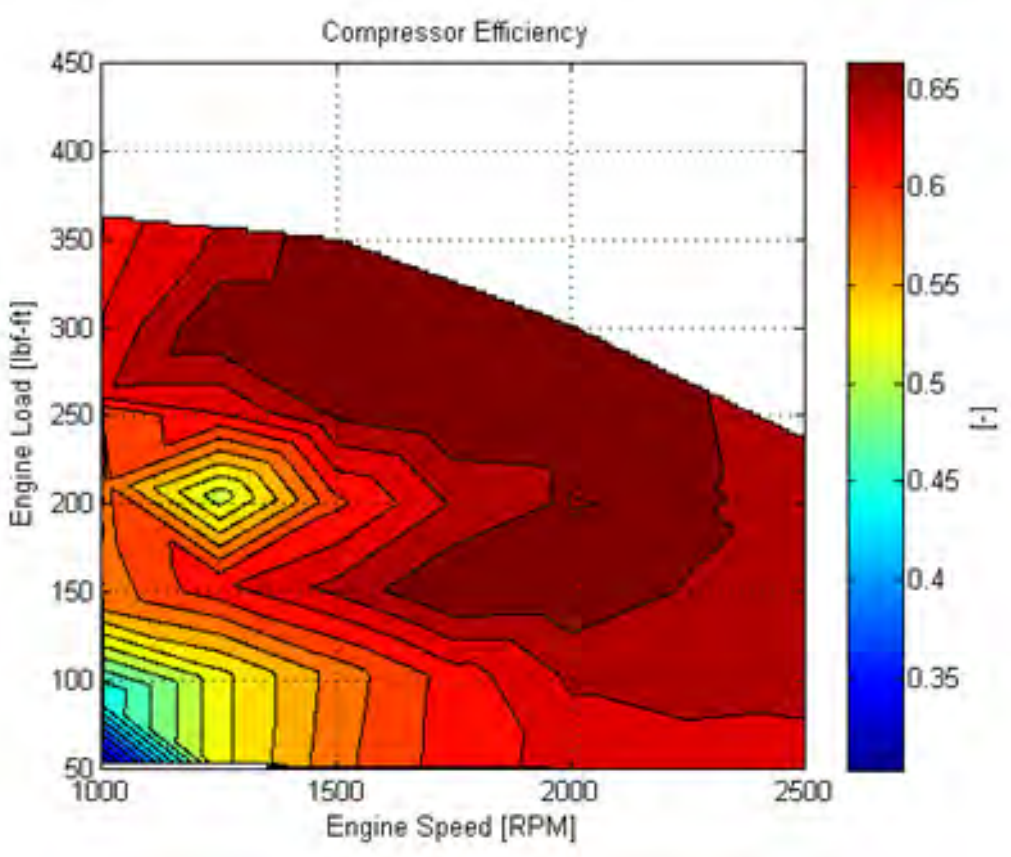

Figure G.5 Engine turbocharger compressor efficiency.

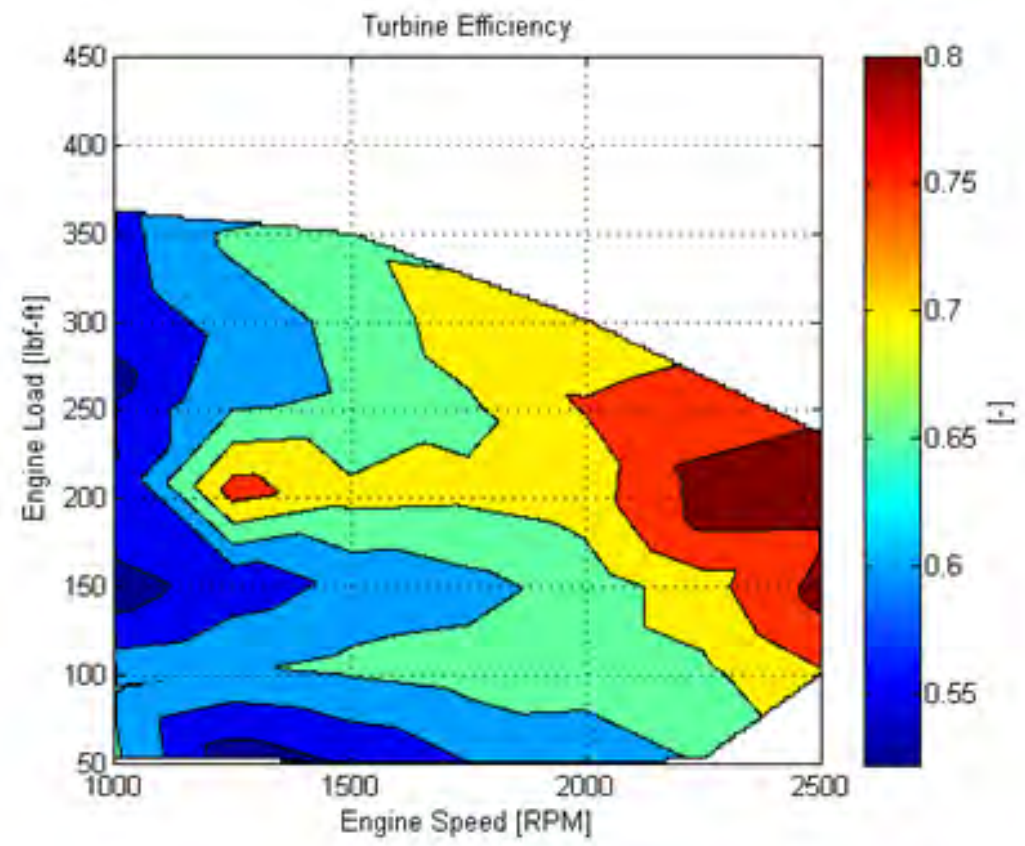

Figure G.6 Engine turbocharger turbine efficiency. 


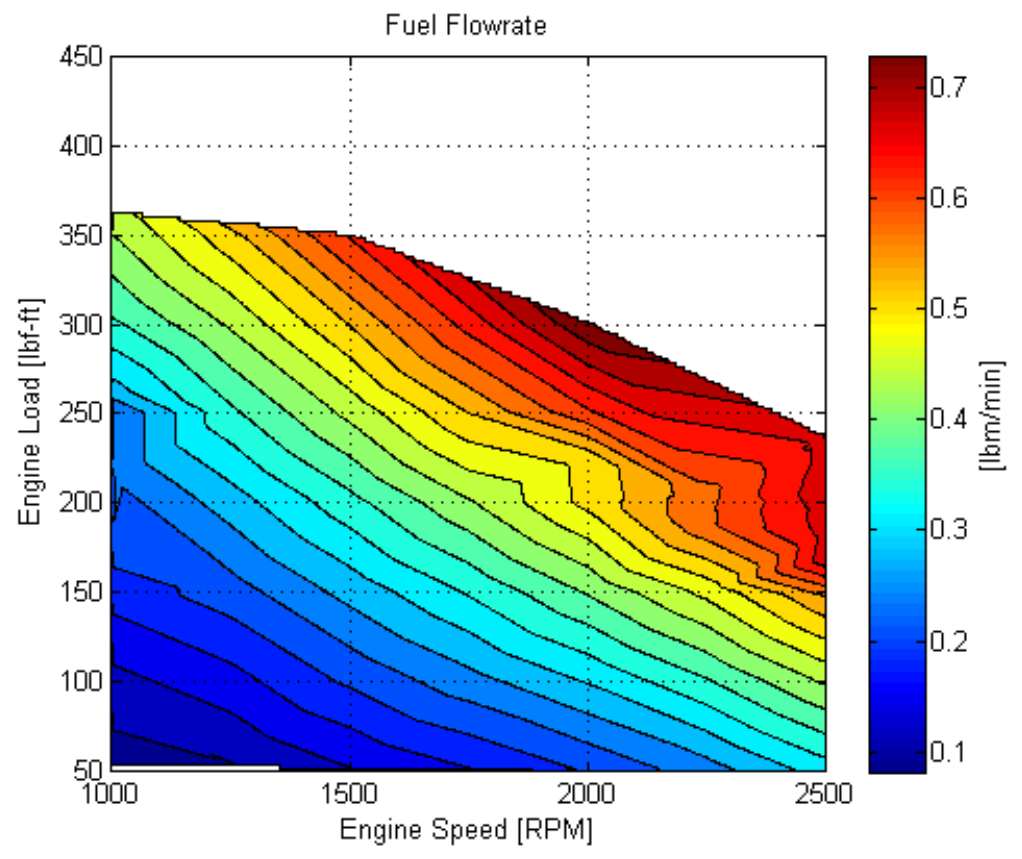

Figure G.7 Engine fuel mass flow rate. 


\section{Appendix H - Sanden R134a Compressor Performance Chart}

Compressor: SD5H14

Refrigerant: R134a

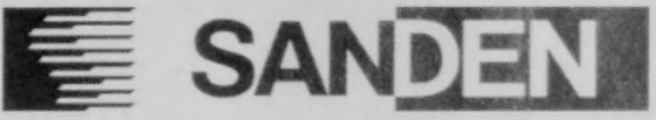

\section{Refrigerating Capacity}

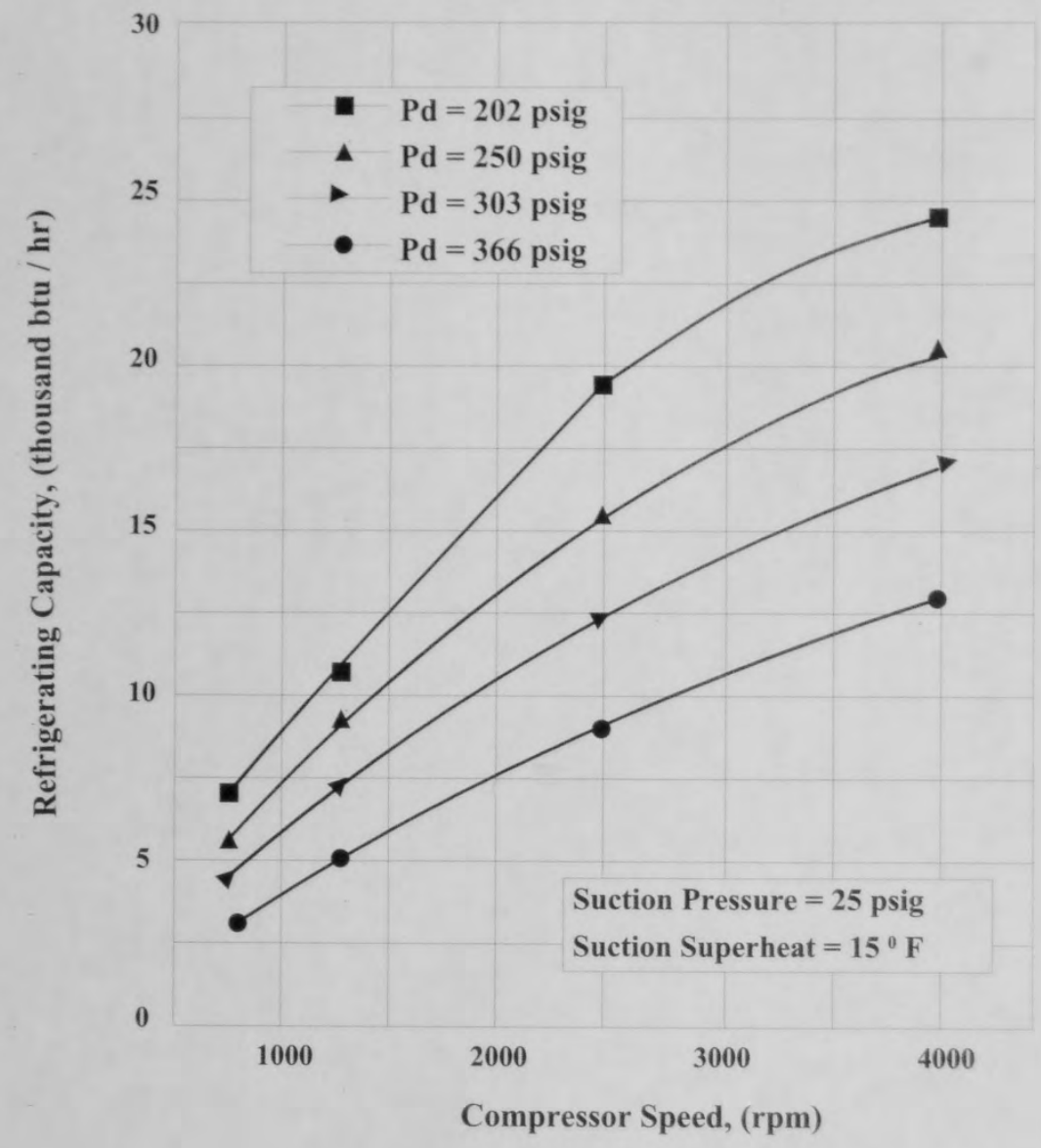

Sanden Engineering Labs

Tested to Standards: ASHRAE 23-78, ISO 917, JS B8606 
Appendix I - ACM Performance Prediction Code in Matlab 
\% Chris Forster

\% This is the main ACM modeling program.

\% 1) Load compressor and turbine data into tab-delimited text files.

$\% 2$ ) Set system parameters (Main_Parameters.m)

\% 3) Execute this file.

\% 4) Everything else is automatic.

$\%$ K

$---$

$\%$ Program starts here.

$\%$

$--$

\% Clear the workspace, command window, close open plots/windows, $\boldsymbol{k}$ etc...

clear;

close all;

clc;

\%matlabpool(2)

\% Load/preprocess compressor data comp_data_process;

\% Load/preprocess turbine data turb_data_process;

\% Load/preprocess heat exchanger data \%HTXR1

$\% \mathrm{n} / \mathrm{a}$ for now

\%HTXR2

htxr2_data_process;

\% Load system parameters

Main_Parameters;

\% Load psychometric table data

PsychometricTables = PsychometricTables $(\odot)$;

\% Find humdity stuff... water_mass_init $=$ PsychometricLookup(ambient.P, ambient.T, ambient. $\boldsymbol{k}$ rel_hum, Psychometrictables) ${ }^{*} 7000 ; \quad \%$ grains

\% T-C Operating Point Matching 
$\%$ Set mass flow rate tolerances and limits

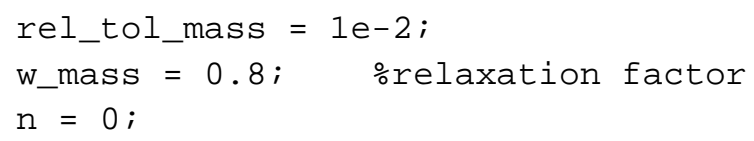

$\%$ Set Initial Guesses

m_dot_c_corr $=6: 0.05: 8$;

PR_c $=1.25: 0.01: 1.55$;

n_calcs $=$ length $\left(m \_d o t \_c \_c o r r\right){ }^{*}$ length $\left(P R \_c\right)$;

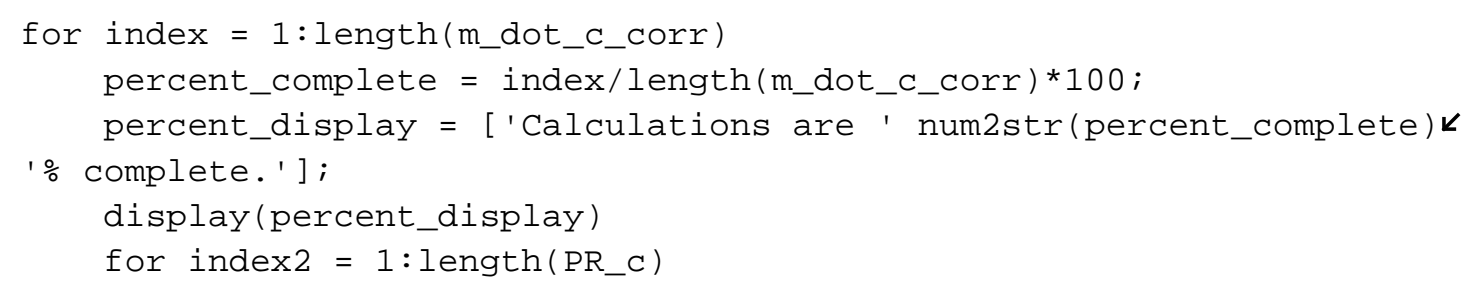


if (abs (multi_acm(keepers_nan(index, 1), keepers_nan(index, 2)). $\boldsymbol{k}$ power_t-multi_acm (keepers_nan $($ index, 1$)$, keepers_nan $($ index, 2) ).power_c $) k$ $<=0.2)$

keepers_power $\left(k \_i n d e x,:\right)=\operatorname{keepers\_ nan}($ index, : $)$;

$\mathrm{k} \_$index $=\mathrm{k} \_$index +1 ;

end

end

k_index $=1$;

for index $=1:$ length $($ keepers_power $(:, 1))$

if (abs (multi_acm(keepers_power(index, 1), keepers_power (index, 2)) . $\boldsymbol{k}$ m_dot_t_phy-multi_acm (keepers_power $($ index, 1$)$, keepers_power $($ index, 2$)) . k$ m_dot_c_phy) $<=0.1)$ keepers_mass $\left(k \_i n d e x,:\right)=$ keepers_power $($ index, : ) ; $\mathrm{k} \_$index $=\mathrm{k} \_$index +1 ;

end

end

figure(2)

subplot $(2,1,2)$

hold on

for index $=1:$ length $\left(\operatorname{keepers} \_\right.$mass $\left.(:, 1)\right)$

scatter(multi_acm(keepers_mass (index, 1), keepers_mass $($ index, 2$)) \cdot \boldsymbol{k}$

P1_t/ambient.P,multi_acm(keepers_power(index, 1 ), keepers_power(index, $\boldsymbol{k}$ 2)).m_dot_t_corrected)

end

figure(2)

subplot $(2,1,2)$

hold on

for index $=1:$ length $\left(\operatorname{keepers} \_\right.$mass $\left.(:, 1)\right)$

scatter(multi_acm(keepers_mass (index,1), keepers_mass (index, 2)).k

P1_t/ambient.P,multi_acm(keepers_power(index, 1 ), keepers_power(index, $\boldsymbol{k}$

2)).eta_t)

end

\% Plots

plots

figure(1)

subplot $(1,2,1)$

hold on 


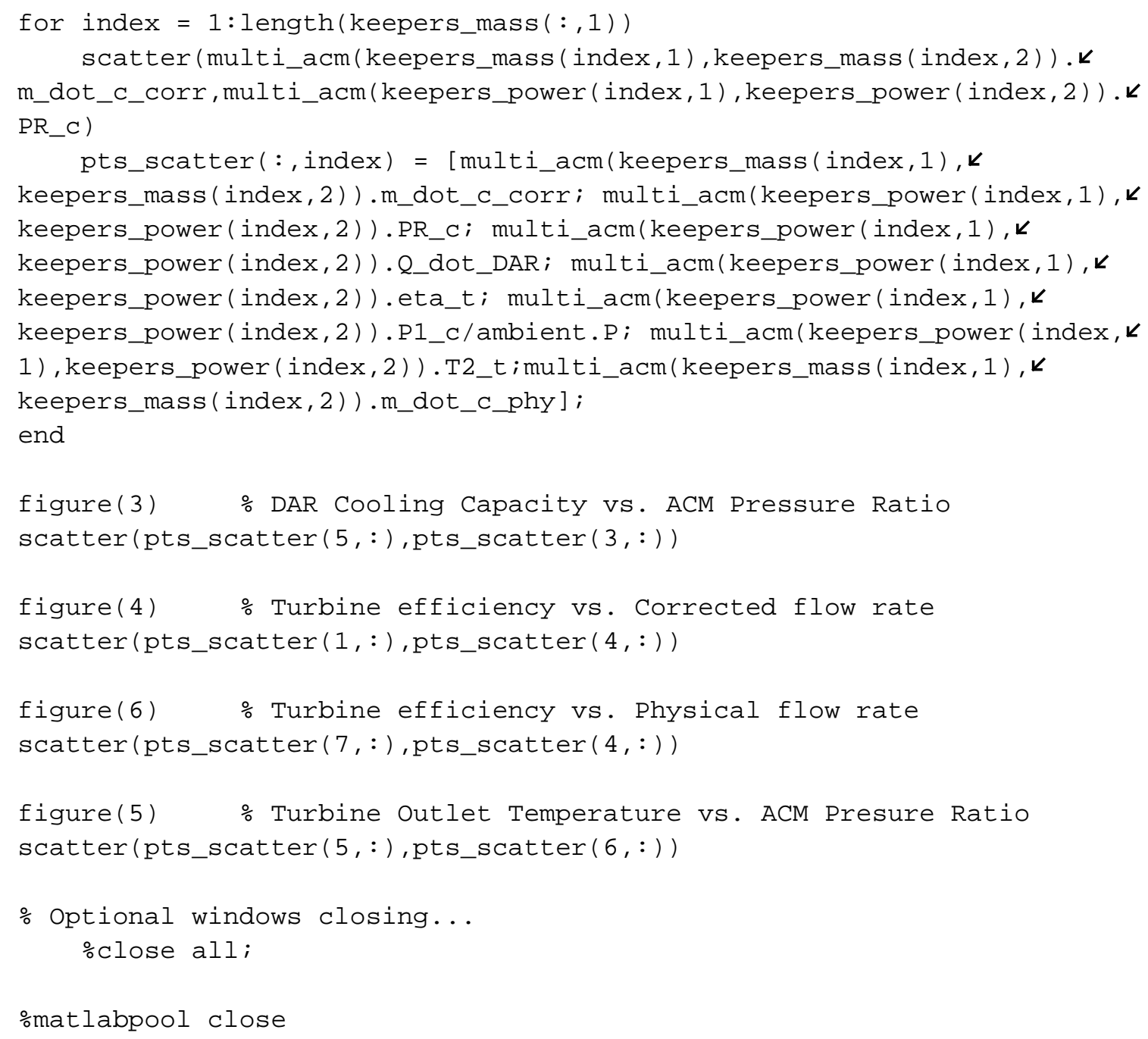




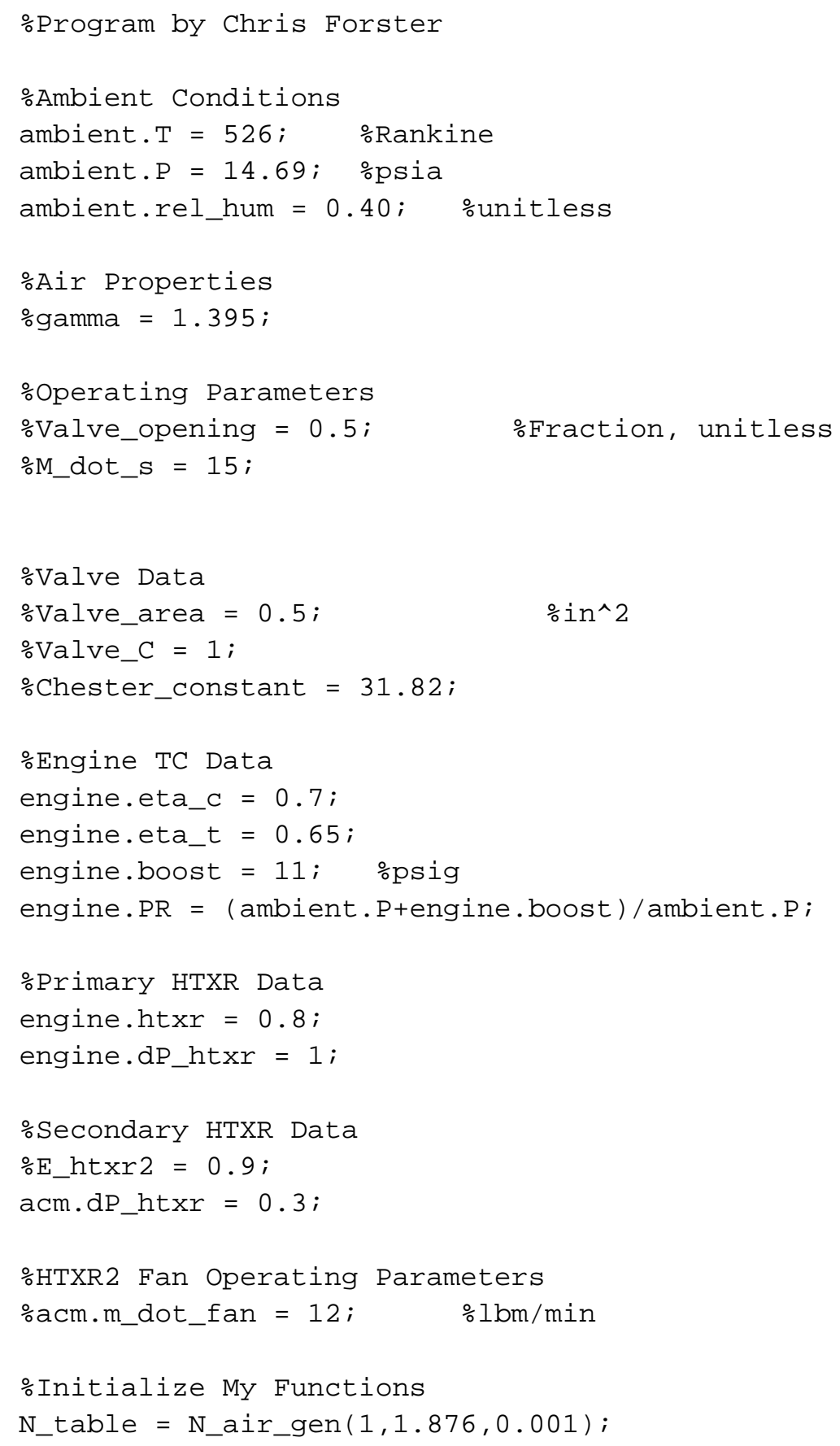


\% Chris Forster

\% Turbine Power

function $\left[W \_t\right]=$ turbine_power $\left(P 1, P 2, T 1, m \_d o t, e t a \_t\right)$

if $($ isnan $(P 1)==1)|(i \operatorname{snan}(P 2)==1)|(i \operatorname{snan}(T 1)==1) \mid$ (isnank

$\left(m \_\right.$dot $\left.)==1\right) \mid($ isnan $($ eta_t $)==1)$

$\mathrm{w}_{\mathrm{L}} \mathrm{t}=0$;

\%display('turbine_power NaN input warning, W_t set to zero!') else

$\mathrm{PR}=\mathrm{P} 1 / \mathrm{P} 2 ;$

C_p $=0.241 ;$

W_t $=m \_d o t{ }^{*} C \_p^{*} T 1^{*}$ eta_t ${ }^{*}((P R \wedge \odot .285-1) / P R \wedge \odot .285){ }^{*} \odot .02358 ; \quad$ \%Units ink $\mathrm{HP}$

end

end \%End of function 
\% Chris Forster

\% Turbine Mass Flow Rate

\%turb_data $(:, 1)=$ Corrected Speed

\%turb_data $(:, 2)=$ Pressure Ratio

\%turb_data $(:, 3)=$ Corrected mass flow rate data

\%turb_data $(:, 4)=$ Turbine Efficiency $(0-1$ range $)$

\%This function returns actual mass flow rate (not corrected).

function[m_dot] = turbine_massflow $\left(P 1, P 2, T 1, N \_p h y, t u r b \_d a t a\right)$

if $($ isnan $(P 1)==1)|(i \operatorname{snan}(P 2)==1)|(i \operatorname{snan}(T 1)==1) \mid($ isnank

(N_phy) $==1$ )

m_dot $=0$;

\%display('turbine_massflow NaN input warning, m_dot set to zero!')

else

$\mathrm{PR}=\mathrm{P} 1 / \mathrm{P} 2 ;$

N_corrected_t $=$ N_phy/sqrt $(T 1 / 519)$;

\% Lookup speeds, then calc two flowrates based on PR

for index $=1:$ length (turb_data.speeds)

if N_corrected_t < turb_data.speeds(1)

m_dot_corrected_t $=0$;

\%display('Corrected Turbine speed Too Low, NO DATA')

break

end

if N_corrected_t $>$ turb_data.speeds(length(turb_data.speeds))

m_dot_corrected_t $=0$;

\%display('Corrected Turbine Speed Too High, NO DATA')

break

end

if N_corrected_t $==$ turb_data.speeds(index)

m_dot_corrected_t $=$ turb_data.fits.flow(index, 1$) k$

${ }^{*} P^{\wedge} 2+t u r b \_d a t a . f i t s . f l o w($ index, 2)*PR+turb_data.fits.flow(index, 3$)$; break

end

if N_corrected_t $<$ turb_data.speeds(index)

m_dot $1=$ turb_data.fits.flow $($ index $-1,1){ }^{*} P R^{\wedge} 2+$ turb_data.fits.k

flow (index-1, 2) *PR+turb_data.fits.flow(index-1, 3);

$\mathrm{m} \_$dot 2 = turb_data.fits.flow $($ index, 1$){ }^{*} P R^{\wedge} 2+$ turb_data.fits.flowk

(index, 2)*PR+turb_data.fits.flow(index, 3);

m_dot_corrected_t $=$ m_dot1 $+\left(\mathrm{m} \_\right.$dot2 $-\mathrm{m} \_$dot 1$) /($ turb_data.speedsk 


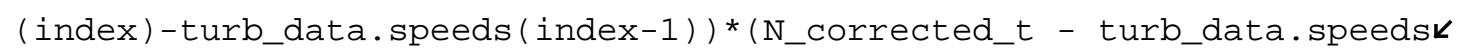
(index-1)); break

end end

$m \_$dot $=$m_dot_corrected_t* $(P 1 * 2.03625437 / 29.92) / \operatorname{sqrt}(T 1 / 519) ;$

end

end \%End of function 
\% Chris Forster

\% This function finds the turbine efficiency from the turbine datak file.

\%turb_data $(:, 1)=$ Corrected Speed

\%turb_data $(:, 2)=$ Pressure Ratio

\%turb_data $(:, 3)=$ Correct Turbine Mass Flow Rate

\%turb_data $(:, 4)=$ Turbine Efficiency

function[eta_turb] = turbine_efficiency $\left(P 1, P 2, T 1, N \_\right.$phy, turb_data $)$

if $($ isnan $(P 1)==1)|(i \operatorname{snan}(P 2)==1)|(i \operatorname{snan}(T 1)==1) \mid$ (isnank

(N_phy) == 1)

eta_turb = 0 ;

\%display('turbine_efficiency NaN input warning, m_dot set tok zero!' )

else

$\mathrm{PR}=\mathrm{P} 1 / \mathrm{P} 2 ;$

N_corrected $=$ N_phy/sqrt $(\mathrm{T} 1 / 519) ;$

\% Lookup speeds, then calc two flowrates based on PR

for index $=1$ : length (turb_data.speeds)

if $N$ _corrected < turb_data.speeds(1)

eta_turb = 0 ;

\%display('Corrected Turbine speed Too Low, NO DATA')

break

end

if N_corrected > turb_data.speeds(length(turb_data.speeds))

eta_turb = 0 ;

\%display('Corrected Turbine Speed Too High, NO DATA')

break

end

if $\mathrm{N} \_$corrected $==$turb_data.speeds (index)

eta_turb $=$ turb_data.fits.eff $($ index, 1$){ }^{*} P R^{\wedge} 2+t u r b \_d a t a . f i t s . e f f k$

(index, 2) *PR+turb_data.fits.eff(index, 3);

break

end

if N_corrected < turb_data.speeds (index)

eta1 = turb_data.fits.eff $($ index $-1,1){ }^{*} P R^{\wedge} 2+t u r b \_d a t a . f i t s . e f f k$

(index-1,2)*PR+turb_data.fits.eff(index-1,3);

eta2 = turb_data.fits.eff $($ index, 1$){ }^{*} P R^{\wedge} 2+$ turb_data.fits.effk

(index, 2)*PR+turb_data.fits.eff(index, 3); 
eta_turb $=$ eta $1+($ eta2-eta1) $/($ turb_data.speeds $($ index $)-\boldsymbol{k}$

turb_data.speeds(index-1))*(N_corrected - turb_data.speeds(index -1$))$; break end

end

end

end \%End of function 


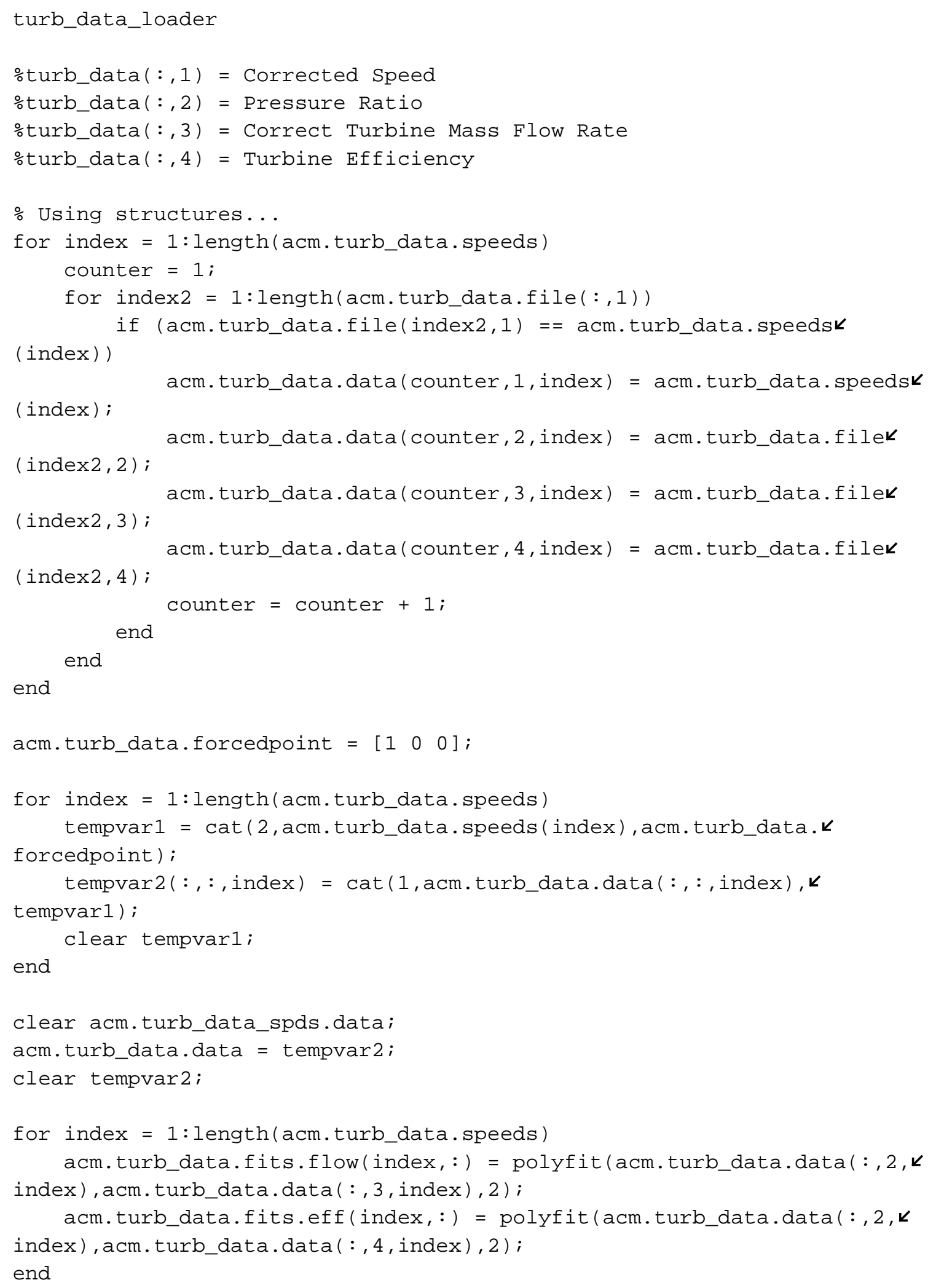


\%Chris Forster

\%The file format is a tab-delimited text file

\%turb_data $(:, 1)=$ Corrected Speed

\%turb_data $(:, 2)=$ Pressure Ratio

\%turb_data $(:, 3)=$ Correct Turbine Mass Flow Rate

\%turb_data $(:, 4)=$ Turbine Efficiency

\%This file will be run from within the simulation (automatically...)

acm.turb_data.file = load('turb_data.txt');

acm.turb_data.speeds = load('turb_speeds.txt'); 
\% Chris Forster

function $[$ acm2 $]=T C \_m a t c h(a c m$, engine, ambient, water_mass_init, $\boldsymbol{k}$ m_dot_c_corr, PR_c)

acm.m_dot_c_corr = m_dot_c_corr ;

acm.PR_c = PR_c;

\% Find Compressor Speed (Physical Speed)

acm.P1_c = engine $\cdot$ boost +14.7 ;

acm.P2_c $=$ acm.P1_c*acm.PR_c;

acm.T1_C $=$ ambient.T-5;

acm.N_c_phy $=$ compressor_speed $($ acm.P1_c,acm.P2_c,acm.T1_c,acm. $k$

m_dot_c_corr,acm.comp_x_speed,acm.comp_y_speed,acm.comp_z_speed);

\% Find physical flow from compressor corrected flow acm.m_dot_c_phy = compressor_flow_phy $\left(\mathrm{acm} . \mathrm{P} 1 \_\mathrm{c}, \mathrm{acm} . \mathrm{T} 1 \_\mathrm{c}, \mathrm{acm} . \boldsymbol{k}\right.$ m_dot_c_corr);

\% Find compressor efficiency.

acm.eta_c $=$ compressor_efficiency $($ acm.P1_c,acm.P2_c,acm.T1_c, $k$ acm.m_dot_c_corr,acm.comp_x_eta,acm.comp_y_eta,acm.comp_z_eta);

\% Find compressor outlet temperature. acm.T2_c $=$ acm.T1_c $+\left(1 / a c m . e t a \_c\right) * a c m . T 1 \_c *\left(\left(a c m . P 2 \_c / a c m . k\right.\right.$ P1_c)^®.285-1); $\quad$ \%Deg R

\% Find required compressor power. acm.power_c = compressor_power $($ acm.P1_c,acm.P2_c, acm.T1_c,acm. $k$ m_dot_c_phy, acm.eta_c);

\% Find pressure drop accross the heat exchanger. acm.P_delta $=0.3 ; \quad \% p s i a$, constant for now... acm.P1_t $=$ acm.P2_c - acm.P_delta;

\% Find heat exchanger outlet temperature. acm.T_delta_htxr2 = acm.T2_c - ambient.T;

acm.E_htxr = 0.83 ;

acm.T1_t $=$ acm.T2_c - acm.E_htxr* (acm.T2_c - ambient.T)；

\% Convert T1_t to T1_t_DAR acm.g_sat_t1 = PsychometricLookup $\left(\operatorname{acm} . P 1 \_t, a c m . T 1 \_t, 1, k\right.$ PsychometricTables)*7000; \%grains acm.T1_t_DAR $=$ T_DAR $\left(\left(\mathrm{acm} . T 1 \_t-460\right), a c m . g \_s a t \_t 1, k\right.$ water_mass_init) +460 ; 
\% Find Turbine mass flow rate (physical) from speed and pressurek ratio across turbine. acm.N_t_phy $=$ acm. N_c_phy;

acm.P2_t = ambient.P;

acm.m_dot_t_phy $=$ turbine_massflow $\left(a c m . P 1 \_t, a c m . P 2 \_t, a c m . k\right.$

T1_t_DAR,acm.N_t_phy,acm.turb_data); \%Actual flow (not corrected)

\% Find corrected turbine flow from m_dot_t acm.m_dot_t_corrected $=$ turbine_massflow_corrected $\left(\operatorname{acm} . P 1 \_t, k\right.$ acm.T1_t_DAR,acm.m_dot_t_phy);

\% Find turbine efficiency acm.eta_t $=$ turbine_efficiency $\left(\mathrm{acm} . \mathrm{P} 1 \_t, a c m . P 2 \_t, a c m . T 1 \_t \_D A R, k\right.$ acm.N_t_phy,acm.turb_data);

\% Find power produced by turbine acm.power_t $=$ turbine_power $\left(a c m . P 1 \_t, a c m . P 2 \_t, a c m . T 1 \_t, a c m . k\right.$ m_dot_t_phy, acm.eta_t);

\% Find turbine outlet temperature

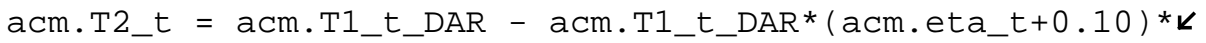
$((($ acm.P1_t/acm.P2_t $) \wedge \odot .285-1) /($ acm.P1_t/acm.P2_t $) \wedge \odot .285)$; \%acm.T2_t $=$ acm.T1_t_DAR - (acm.power_t*42.4072263) $/ \mathrm{acm} . \boldsymbol{k}$ m_dot_t_phy/0.241;

\% Find humdity stuff... acm.T2_g_sat = PsychometricLookup $($ ambient.P, acm.T2_t $, 1, k$ Psychometrictables)*7000; \%grains acm.T2_t_DAR = T_DAR( (acm.T2_t -460), acm.T2_g_sat, $\boldsymbol{k}$ water_mass_init) +460 ; acm.T2_t_DB $=$ T_DAR_DB ( (acm.T2_t_DAR-460), acm.T2_g_sat, $k$ water_mass_init) +460 ;

\% Find cooling capacity acm.Q_dot_DAR $=$ acm.m_dot_t_phy* $0.241^{*}($ ambient.$T$ - acm. $\boldsymbol{k}$ T2_t_DAR) ${ }^{*} 60 / 12000 ; \quad$ \%Units in tons

\% Cleanup time...

clear index;

clear index2;

clear counter;

$\mathrm{acm} 2=\mathrm{acm} ;$

end \%End of function 
\%Program by Chris Forster

\%July 15, 2008

\%Input all humidity ratios in grains/lbm

\%Input temperature in degrees $\mathrm{F}$

\%Returns Dry Air Rated Temperature in degrees $\mathrm{F}$

function [T_DB] = T_DAR_DB (T_DAR,g_sat,g_tot $)$

if $($ isnan $($ T_DAR $)==1) \mid($ isnan $($ g_sat $)==1) \mid($ isnan $($ g_tot $)==1)$ T_DB $=0$;

\%display('T_DAR_DB NaN input warning, T_DB set to zero!') else

$\%$ Define g_ent (entrained water)

if (g_tot $>=$ g_sat)

g_ent = g_tot - g_sat;

else

end

g_ent $=0$;

\% Convert grains to $1 \mathrm{bm}$

g_sat $=$ g_sat $/ 7000$;

g_tot $=$ g_tot $/ 7000$;

g_ent $=$ g_ent $/ 7000$;

\% Calculate T_DB from T_DAR assuming above 32F (DB)

\% Check assumption afterwards.

T_DB_TBD $=\left(T \_D A R^{*}\left(0.24+0.4332{ }^{*}\right.\right.$ g_tot $)+1093^{*}$ g_ent $) /(0.24+0 . k$ $4332^{*}$ g_sat+g_ent);

if (T_DB_TBD $<=32$ ) T_DB_TBD $=\left(\right.$ T_DAR ${ }^{*}\left(0.24+0.4332^{*}\right.$ g_tot $)+1221^{*}$ g_ent $) /(0.24+0 . k$ $4332 *$ g_sat $+0.5^{*}$ g_ent );

end

T_DB $=$ T_DB_TBD; $\quad$ \%Degree $\mathrm{F}$

end

end 


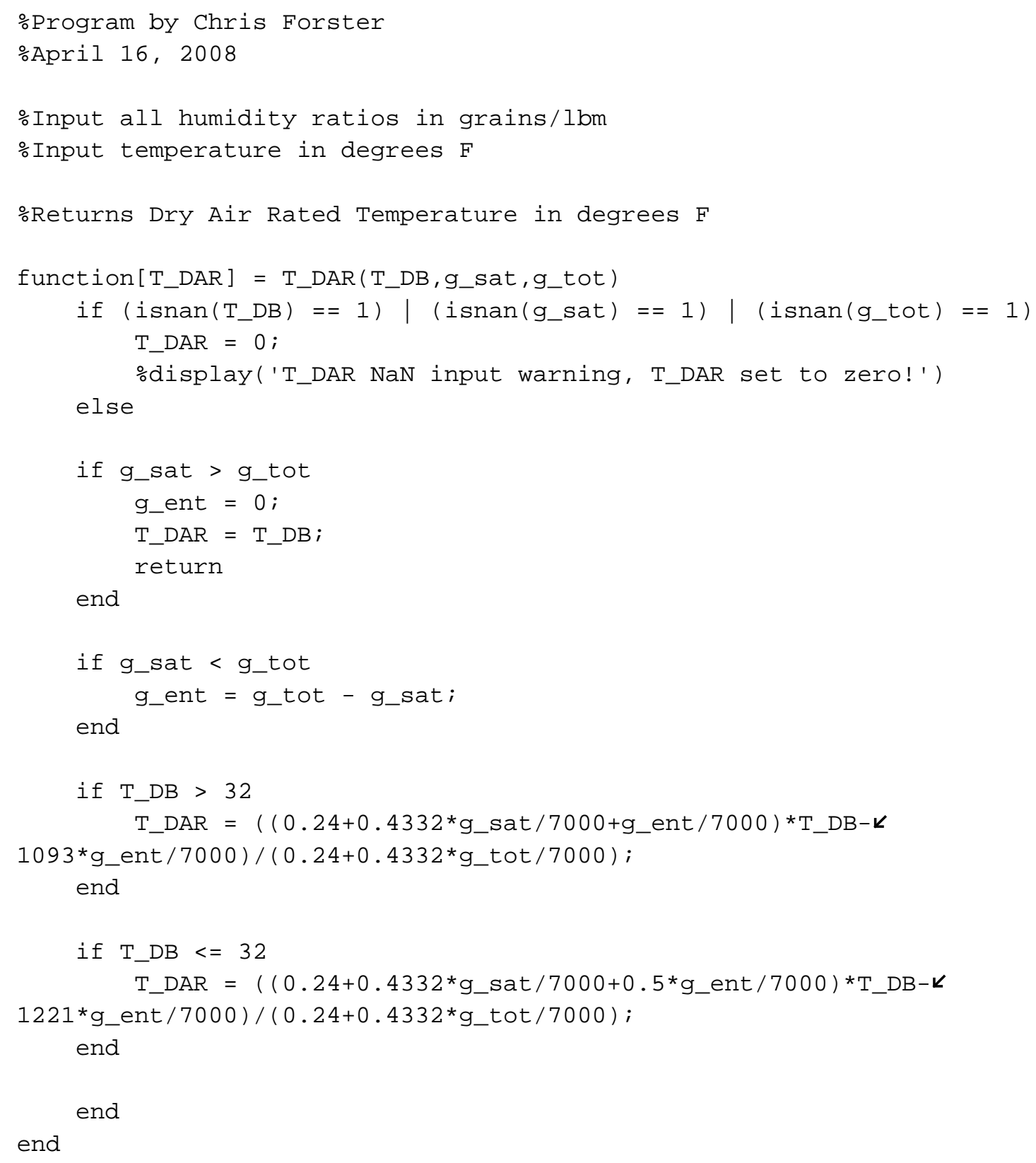




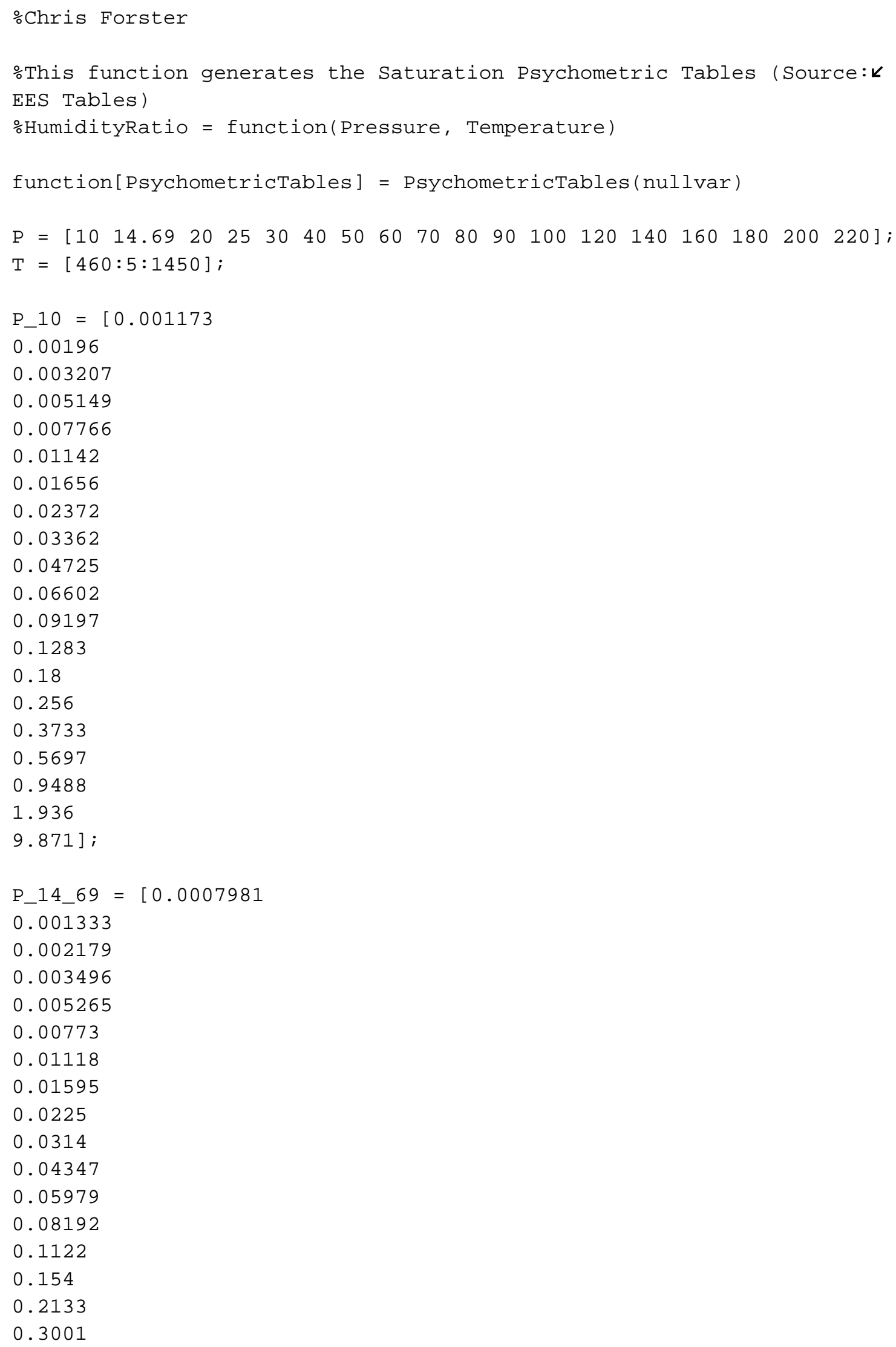




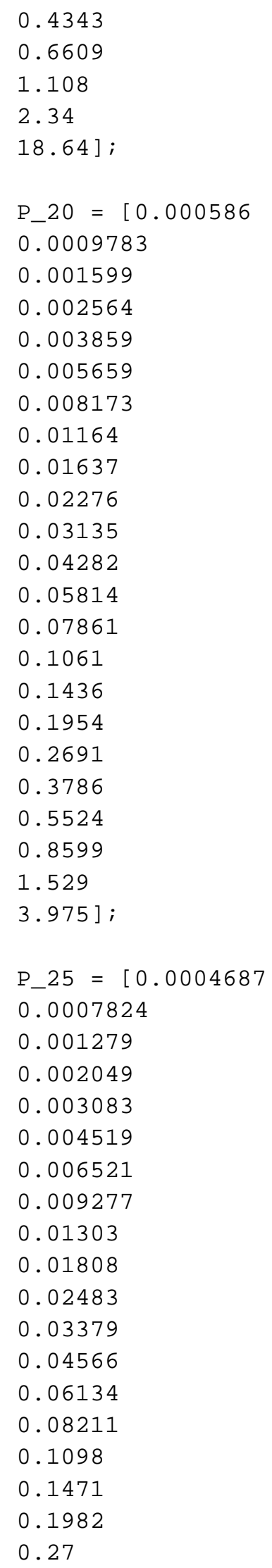




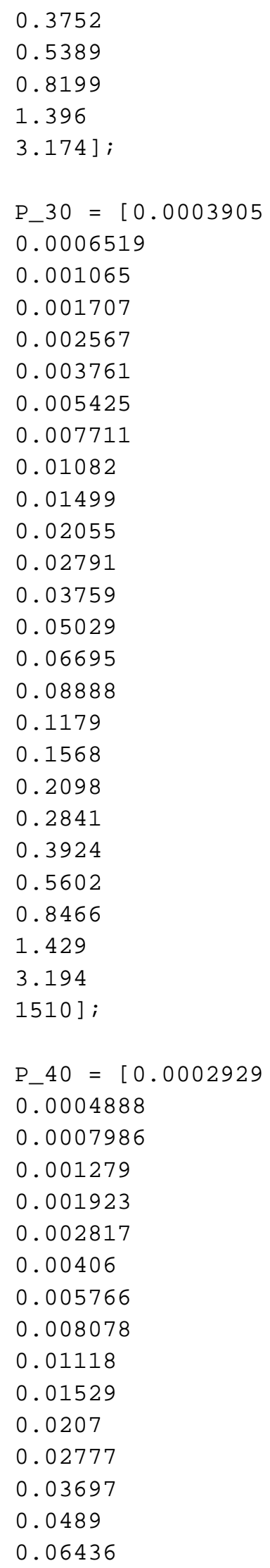




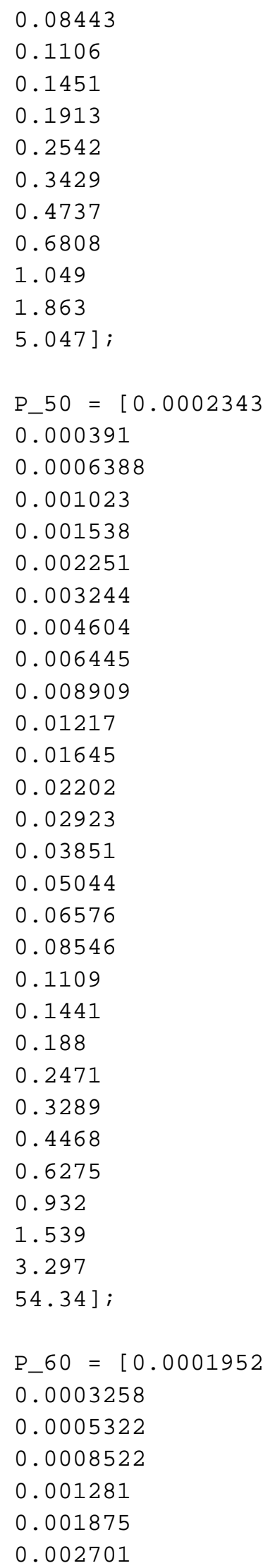




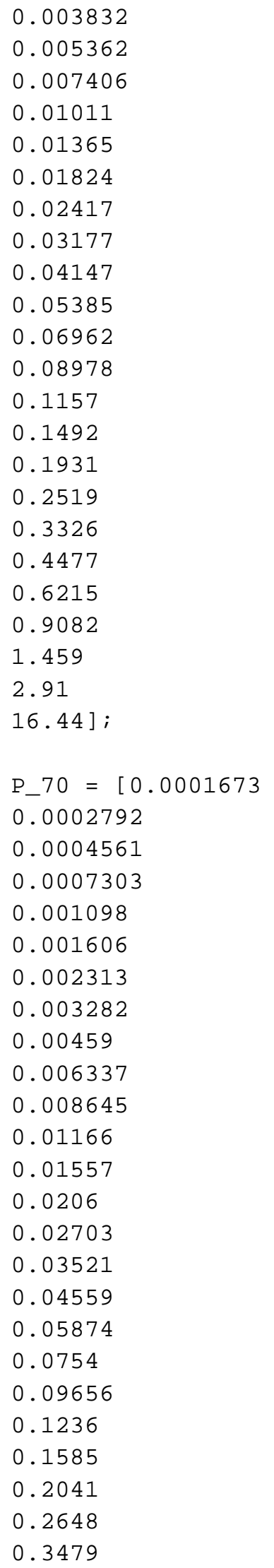




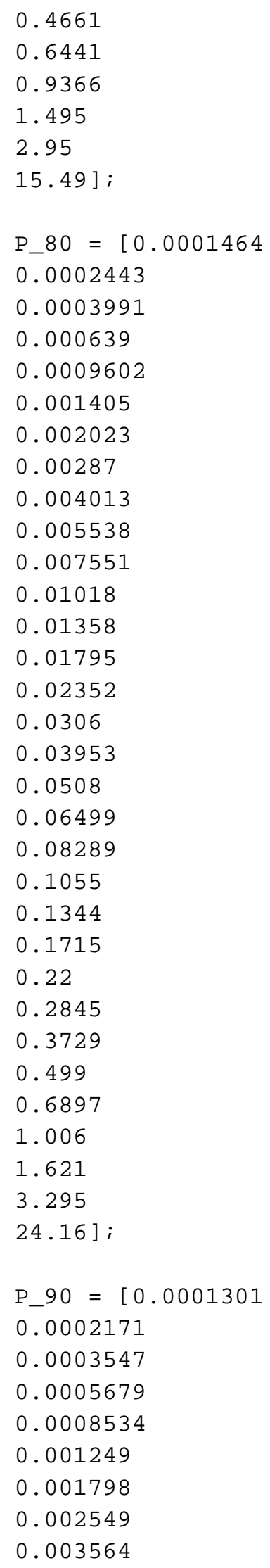




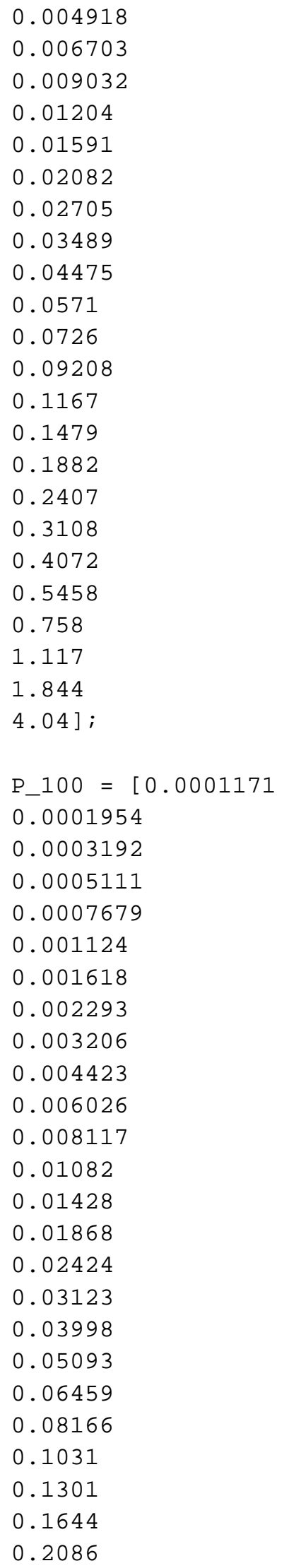




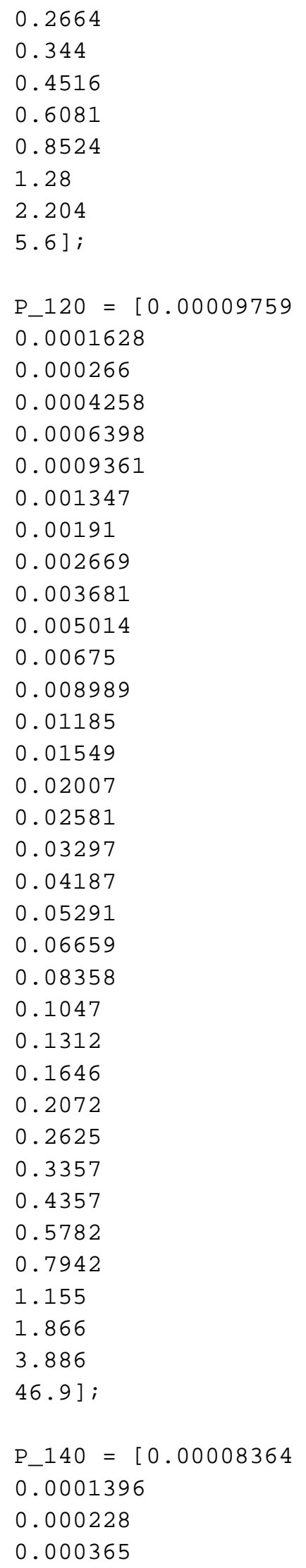




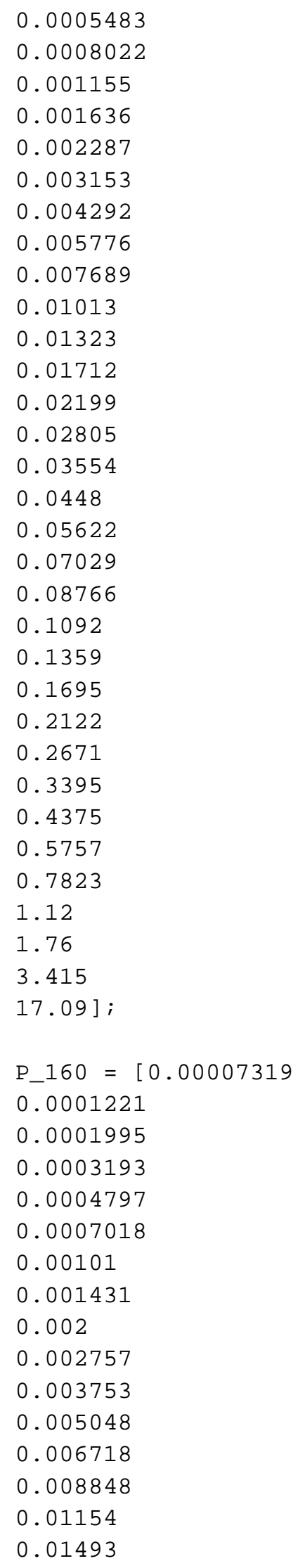




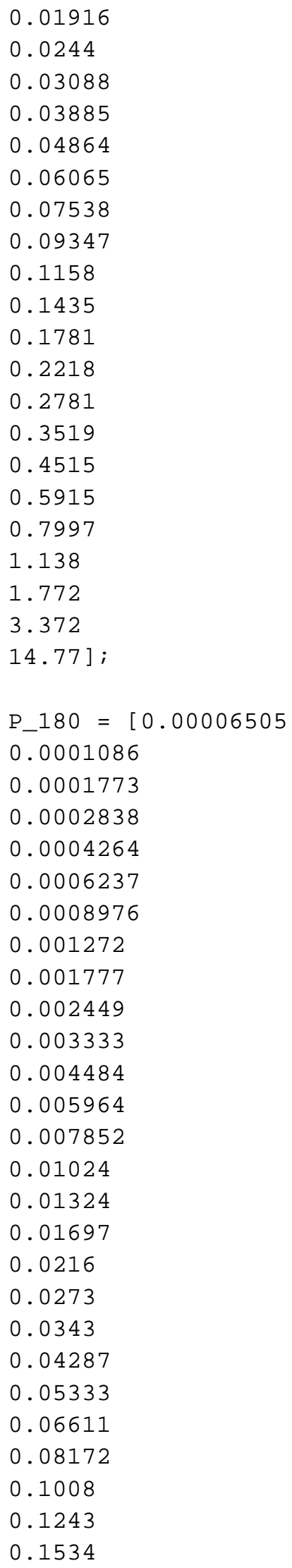

0.1534 


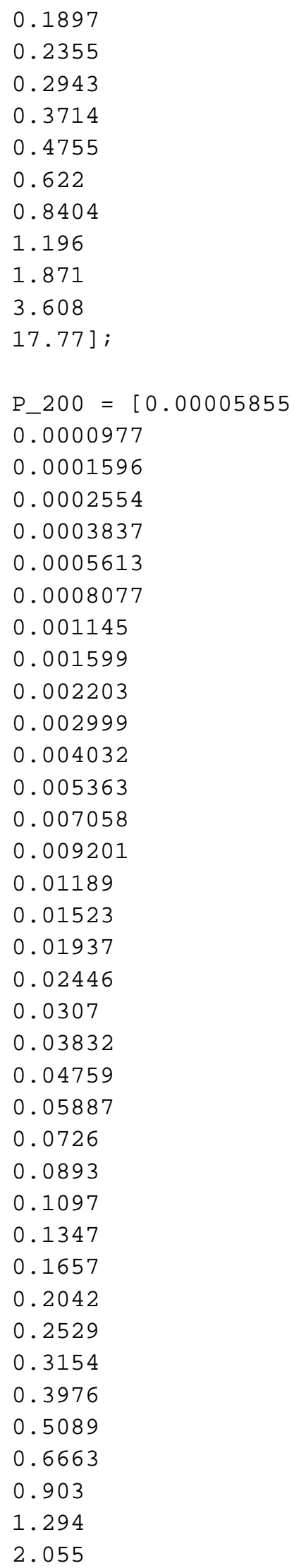




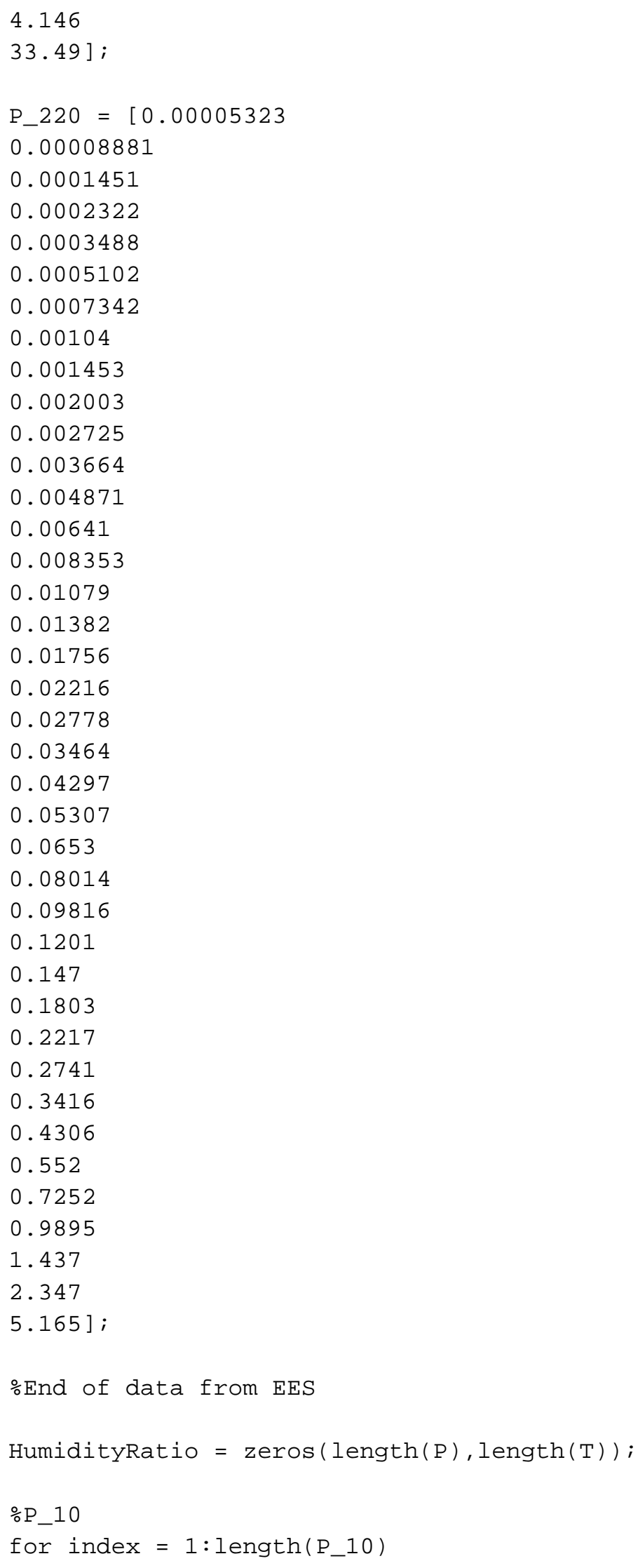

0.2741

$\odot .3416$

0.4306

0.552

0.7252

0.9895

1.437

2.347

$5.165]$

\%End of data from EES

HumidityRatio $=\operatorname{zeros}($ length $(\mathrm{P}), \operatorname{length}(\mathrm{T}))$;

\%P_10

for index $=1:$ length $\left(P \_10\right)$ 


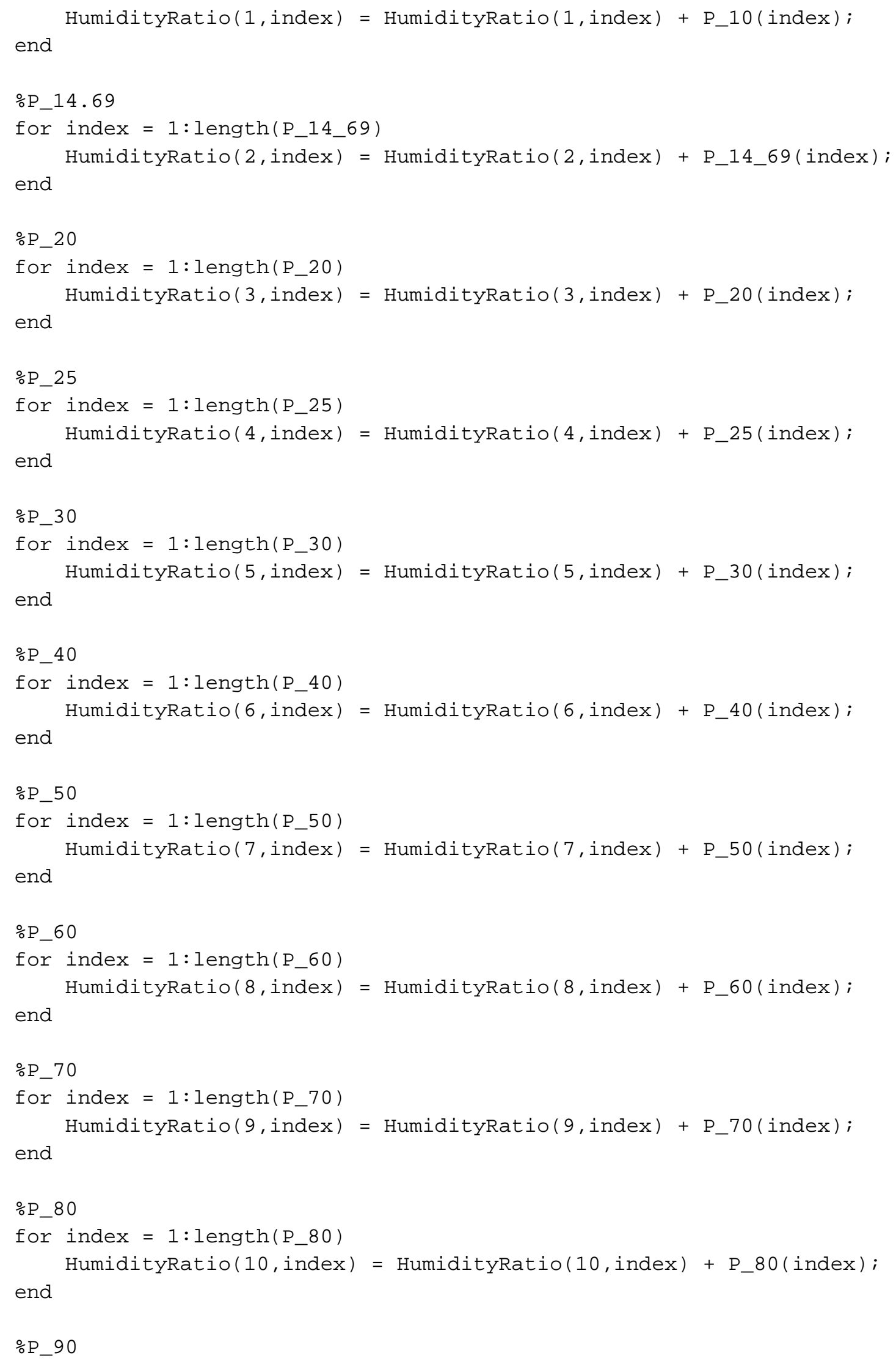




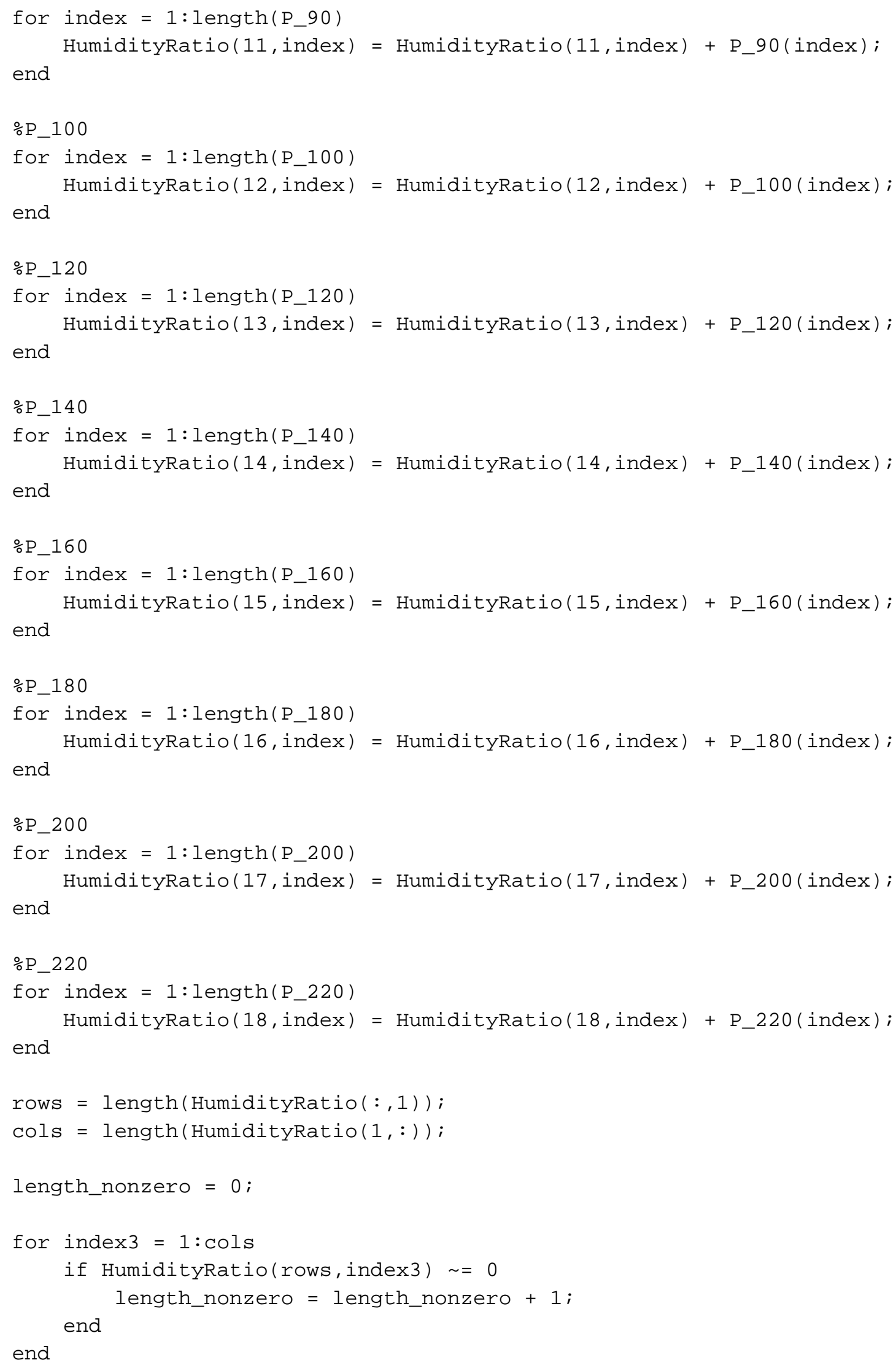




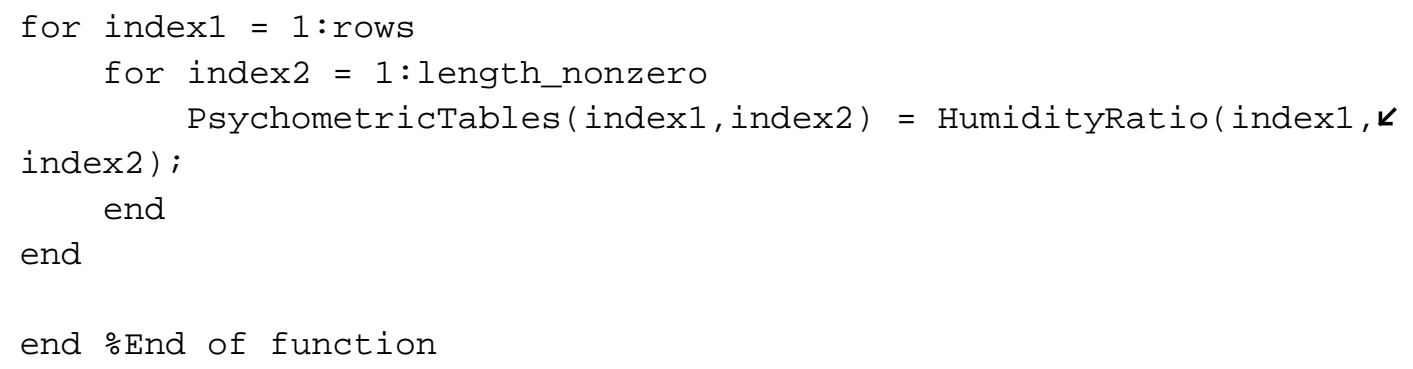


\%Chris Forster

\% Temperature - Rankine

$\%$ Pressure - psia

function[PsychometricLookup_output] = PsychometricLookup(Pressure, $\boldsymbol{k}$ Temperature, RelativeHumidity, PsychometricTables)

$\mathrm{P}=\left[\begin{array}{llllllllllllllllll}10 & 14.69 & 20 & 25 & 30 & 40 & 50 & 60 & 70 & 80 & 90 & 100 & 120 & 140 & 160 & 180 & 200 & 220\end{array}\right]$;

$T=[460: 10: 460+38 * 10]$;

PsychometricLookup_sat $=$ interp2 $(T, P, P s y c h o m e t r i c T a b l e s$, Temperature, $\boldsymbol{k}$ Pressure, 'spline');

PsychometricLookup_output = PsychometricLookup_sat ${ }^{*}$ RelativeHumidity;

end \%End of function 


\section{\%Chris Forster}

$\%$ This section of code is for plotting...keeps it clean.

$\%$ Get screensize and figure layout

acm. chart. screensize $=\operatorname{get}\left(\odot,{ }^{\prime} \operatorname{Screensize}{ }^{\prime}\right)$;

\%Figure(1) location and size

acm. chart.comp_x_loc $=0$;

acm. chart. comp_y_loc $=0$;

acm. chart.comp_width $=$ acm. chart.screensize $(3)$;

$\mathrm{acm}$. chart. comp_height $=$ acm. chart.screensize $(4) * 0.95$;

acm. chart.comp_pos $=[$ acm. chart.comp_x_loc, acm. chart.comp_y_loc, $\boldsymbol{k}$ acm.chart.comp_width, acm.chart.comp_height];

\%Figure(2) location and size

acm. chart.turb_x_loc $=$ acm. chart.screensize $(3) / 3$;

acm. chart.turb_y_loc $=$ acm. chart.screensize $(4) * 2 / 3$;

acm. chart.turb_width $=$ acm. chart.screensize $(3) / 3$;

acm. chart.turb_height $=$ acm. chart.screensize $(4) * 0.3$;

acm.chart.turb_pos $=$ [acm.chart.turb_x_loc, acm.chart.turb_y_loc, $k$ acm.chart.turb_width, acm.chart.turb_height];

\% Compressor Plots

figure('Position', acm. chart.comp_pos)

figure(1)

subplot $(1,2,1)$

contourf(acm.comp_x_eta,acm.comp_y_eta,acm.comp_z_eta)

title 'Compressor Efficiency'

xlabel 'Corrected Mass Flow Rate [1bm/min]'

ylabel 'Pressure Ratio [-]'

colorbar

hold on

PR_c ) ;

comp_point $=$ plot $\left(m u l t i \_a c m(1) \cdot m \_d o t \_c \_c o r r, m u l t i \_a c m(1) \cdot k\right.$

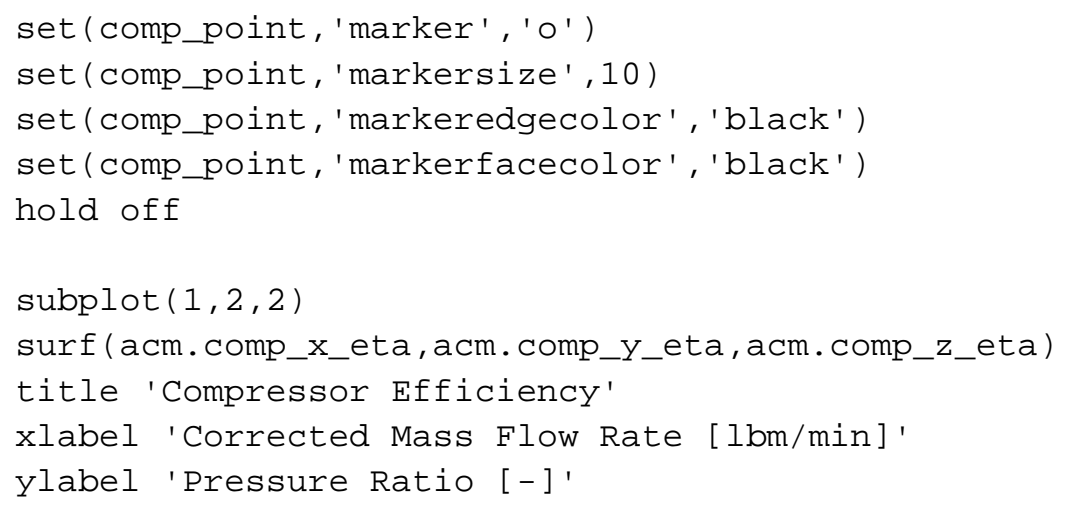


\% Turbine Plots

figure('Position', acm.chart.turb_pos)

figure(2)

subplot $(2,1,1)$

for index $=1$ : length (acm.turb_data.speeds)

for index $2=1:$ length $\left(\operatorname{acm} . t u r b \_d a t a \cdot \operatorname{data}(:, 1,1)\right)-1$

$\mathrm{acm}$. chart.turbine.data $($ index 2,1 , index $)=\mathrm{acm} \cdot \boldsymbol{k}$

turb_data.data(index2,1,index);

$\mathrm{acm}$.chart.turbine.data (index 2,2 , index $)=\mathrm{acm} . \mathrm{k}$

turb_data.data(index2,2, index);

acm. chart.turbine.data (index 2,3, index $)=$ acm. $k$

turb_data.data(index2,3, index);

acm. chart.turbine.data $($ index 2,4 , index $)=$ acm. $k$

turb_data.data(index2,4, index);

end

end

for index $=1:$ length (acm.turb_data.speeds $)$

hold all

x_plot $=$ acm.chart.turbine.data $(:, 2$, index $)$;

y1_plot $=$ acm. chart.turbine.data $(:, 3$, index $)$;

y2_plot $=$ acm.chart.turbine.data $(:, 4$, index $)$;

$[A X, H 1, H 2]=p l o t y y\left(x \_p l o t, y 1 \_p l o t, x \_p l o t, y 2 \_p l o t\right.$, 'plot ' $)$;

hold off

clear x_plot;

clear y1_plot;

clear y2_plot;

\%legend('a', 'b', 'c', 'd', 'location', 'eastoutside' )

$\operatorname{set}\left(A X(1), ' x l i m ',\left[\begin{array}{ll}1 & 2.4\end{array}\right]\right)$

set (AX(1), 'ylim', [ [ 8 8 $]$ )

$\operatorname{set}(A X(2), ' x \lim$ ', [1 1 2.4])

set $\left(\operatorname{AX}(2)\right.$, 'ylim', $\left.\left[\begin{array}{lll}0.4 & 1.2\end{array}\right]\right)$

set(H1(1), 'color', get_color(index))

set(H2(1), 'color', get_color(index))

set (H1(1), 'linewidth', 2)

set (H2(1), 'linewidth', 2)

$\operatorname{set}\left(\operatorname{AX}(1),{ }^{x}\right.$ tick', $\left.[1: 0.2: 2.4]\right)$

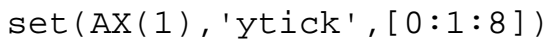

$\operatorname{set}(\operatorname{AX}(2)$, 'ytick', $[0.4: 0.1: 1.2])$

set( $A X(1)$, 'xminorgrid', 'on') 


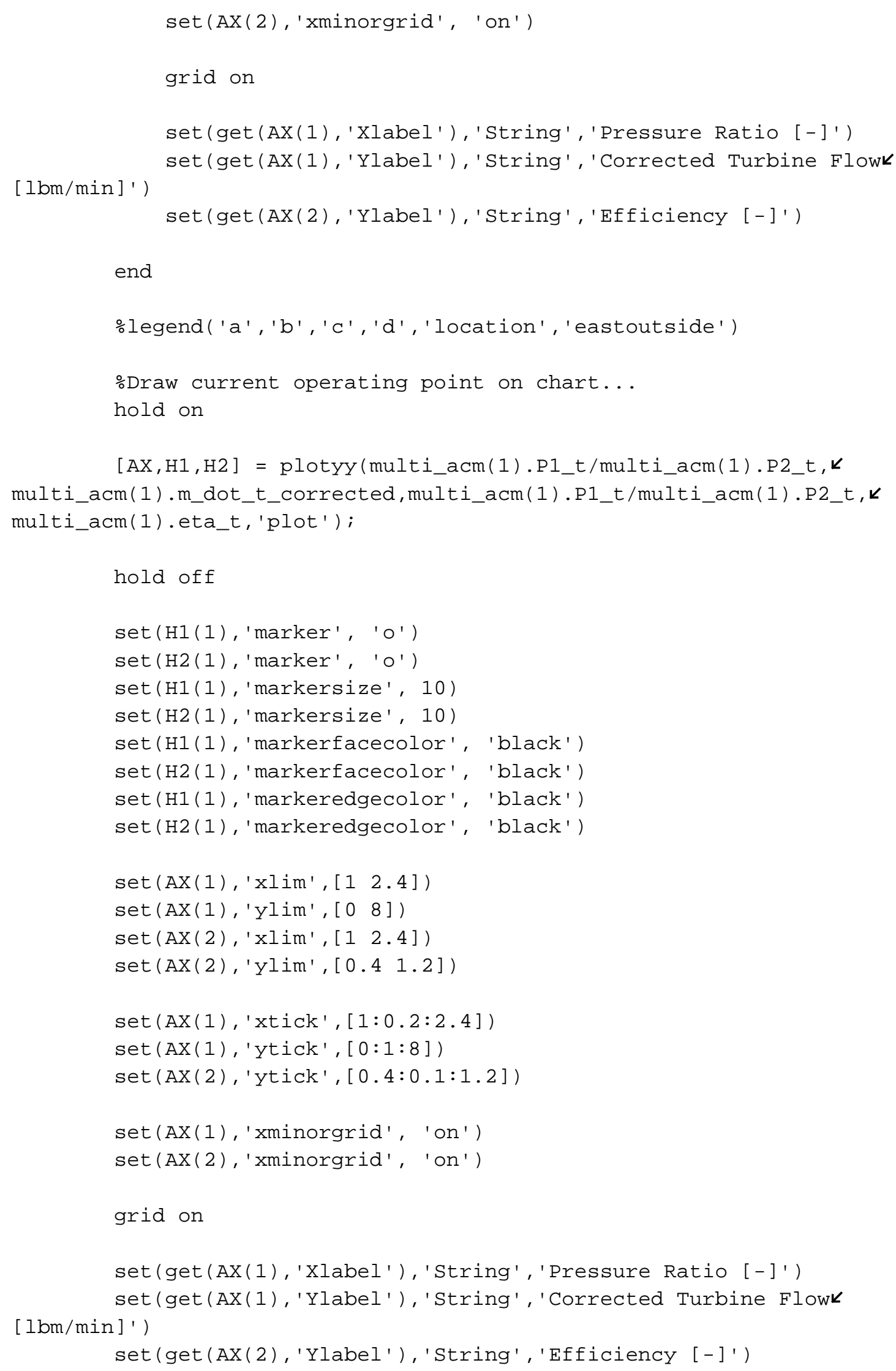




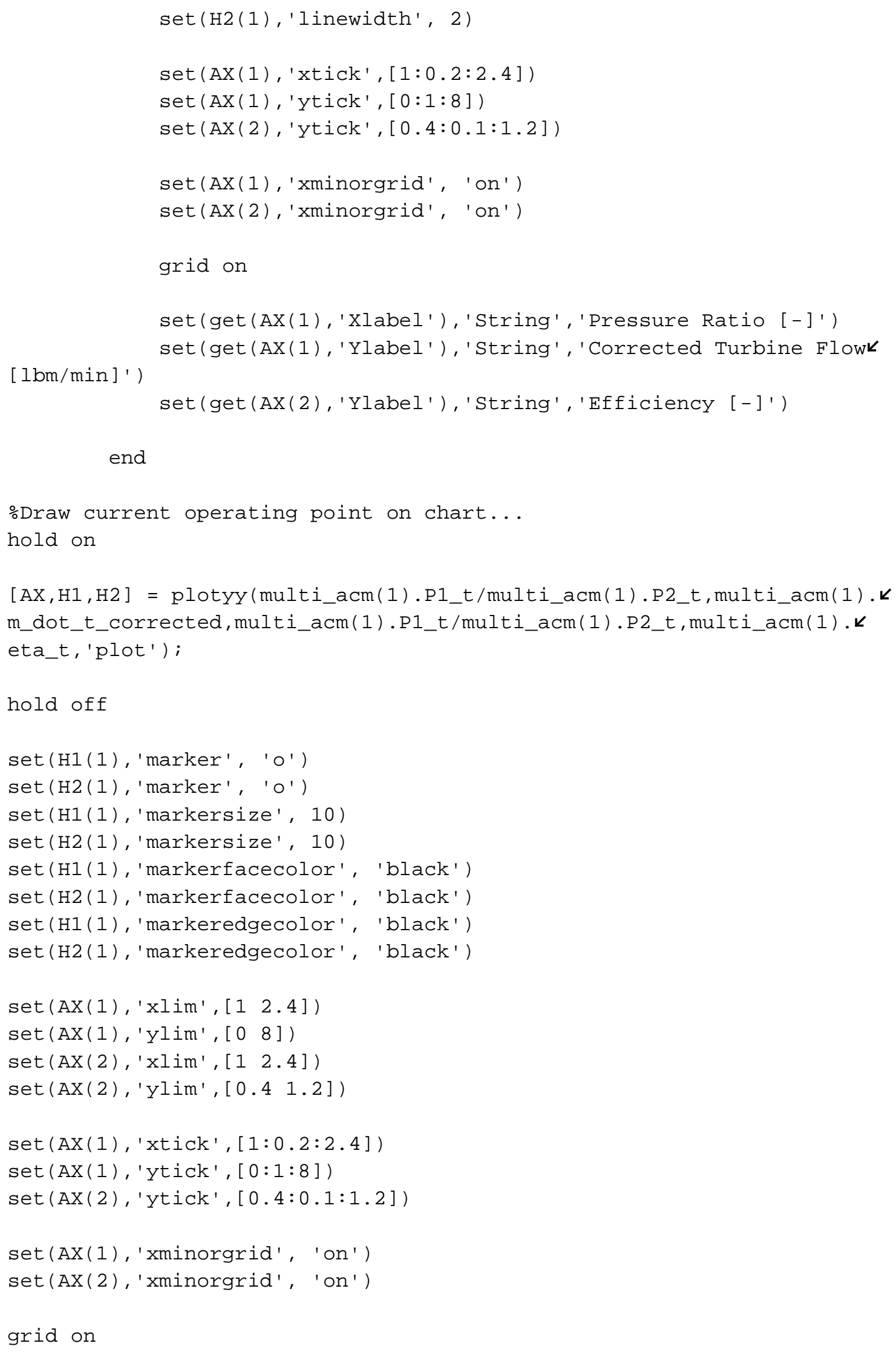


set (get(AX(1), 'Xlabel'), 'String', 'Pressure Ratio [-]')

set (get(AX(1), 'Ylabel'), 'String', 'Corrected Turbine Flow [lbm/min]')

set (get (AX(2), 'Ylabel'), 'String', 'Efficiency [-]')

$\%$ End of current operating points 
\%Program by Chris Forster

\%April 8, 2008

function[PressureRatio] = N_air_rev(N_in, N)

\%Isentropic Exponent Lookup and Interpolation

\%Reference - Chester Smith

\%Accuracy of function is good. The largest difference between thek function and Chester Smith's tables I've seen so far is

\%approximately 0.0003

\%table_resolution should be a value like $\odot .01$ or $\odot .001$, depending onk the

\%table entry step size you want...more means slower, but morek accurate...

\%Returns error if you specify table_start less than one

if $\left(\mathrm{N} \_\right.$in $<=\mathrm{N}(1$, length $\left.(\mathrm{N}(2,:)))\right)$

PressureRatio = interp1 $(\mathrm{N}(2,:), \mathrm{N}(1,:)$, N_in, 'spline' $)$;

else

PressureRatio $=0.00000001 ;$

end

end 
\%Program by Chris Forster

\%April 8, 2008

function[N_air_gen] = N_air_gen(table_start, table_end, $\boldsymbol{k}$

table_resolution)

\%Isentropic Exponent Lookup and Interpolation

\%Reference - Chester Smith

\%Accuracy of function is good. The largest difference between thek function and Chester Smith's tables I've seen so far is

\%approximately 0.0003

\%table_resolution should be a value like $\odot .01$ or 0.001 , depending onk the \%table entry step size you want...more means slower, but morek accurate...

\%Returns error if you specify table_start less than one

if (table_start $<1$ )

N_air_gen $=$ 'error - table_start less than 1';

end return

if (table_end $<=$ table_start) N_air_gen = 'error - check table bounds'; return

end

$\mathrm{P} 2=14.69 ;$

$C 1=[2.06806,1 ; 2.06416,1.0500 ; 2.004159781,1.879] ;$

PR = table_start:table_resolution:table_end;

$\mathrm{P} 1=\mathrm{P} 2{ }^{*} \mathrm{PR} ;$

for index $=1:$ length $(P R)$

if $(\mathrm{PR}($ index $)<=\mathrm{C} 1(3,2))$

N_air_gen $(1$, index $)=P R($ index $)$;

N_air_gen $(2$, index $)=\operatorname{sqrt}(P 2 *(P 1($ index $)-P 2)) / P 1($ index $)$ *interp1k

$(\mathrm{C} 1(:, 2), \mathrm{C} 1(:, 1), \mathrm{PR}($ index $)$, 'linear' $)$; else

N_air_gen $(1$, index $)=P R($ index $)$;

end

$N \_a i r \_g e n(2$, index $)=1$;

end

end 
\%Program by Chris Forster

\%April 8, 2008

function $\left[\mathrm{N} \_\right.$air $]=\mathrm{N} \_$air $\left(\mathrm{PR} \_\right.$in, $\left.\mathrm{N}\right)$

\%Isentropic Exponent Lookup and Interpolation

\%Reference - Chester Smith

\%Accuracy of function is good. The largest difference between thek function and Chester Smith's tables I've seen so far is

\%approximately 0.0003

\%table_resolution should be a value like $\odot .01$ or $\odot .001$, depending on the \%table entry step size you want...more means slower, but morek accurate...

\%Returns error if you specify table_start less than one

if $\left(P R \_\right.$in $\left.<1\right)$

N_air $=$ 'error - PR less than $1 \ldots$ reverse flow probably notk correct '

return

end

if (PR_in $<=N(1$, length $(N(1,:))))$

$\mathrm{N} \_$air $=$interp1 $\left(\mathrm{N}(1,:), \mathrm{N}(2,:), \mathrm{PR} \_\right.$in, 'spline' $)$;

else

N_air = 1;

end

end 
\% Chris Forster

$\%$ This function finds the turbine speed.

function [speed] = get_speed $($ index, acm $)$

speed $=$ acm.turb_data.speeds (index);

end \%End of function 
\% Chris Forster

\% This function allows indexed colors for easy plot formatting, avoidsk adjacent duplicate or hard-to-see colors.

function $[$ color $]=$ get_color $($ index $)$

switch index

case $\{1\}$

color = 'blue';

case $\{2\}$

color = 'red';

case $\{3\}$

color = 'green';

case $\{4\}$ color = 'cyan';

case $\{5\}$ color = 'magenta';

case $\{6\}$ color = 'black';

case $\{7\}$ color = 'blue';

case $\{8\}$

color = 'red';

case $\{9\}$

color = 'green';

case $\{10\}$ color = 'cyan';

case $\{11\}$ color = 'magenta';

case $\{12\}$ color = 'black';

otherwise

disp('Too many speeds... expand color range.'); color $=0$;

end

end \%End of function 
\% Chris Forster

\% Compressor Outlet Temperature

function $[\mathrm{T} 2]=$ compressor_temp $(\mathrm{P} 1, \mathrm{P} 2, \mathrm{~T} 1$, comp_data $)$

$\mathrm{PR}=\mathrm{P} 2 / \mathrm{P} 1$

$\mathrm{T} 2=\mathrm{T} 1+\mathrm{T} 1 /$ comp_data* $\left(\mathrm{PR}^{\wedge} \odot .283-1\right) ;$

end \%End of function 
\% Chris Forster

\% Compressor Speed

\%comp_data $(:, 1)=$ Corrected Speed

\%comp_data $(:, 2)=$ Corrected mass flow rate data

\%comp_data $(:, 3)=$ Pressure Ratio

$\%$ This function returns the physical speed (not corrected)

function [N_phy] = compressor_speed $\left(\mathrm{P} 1, \mathrm{P} 2, \mathrm{~T} 1, \mathrm{~m} \_\right.$dot, comp_X_speed, $\boldsymbol{k}$ comp_y_speed, comp_z_speed)

$\mathrm{PR}=\mathrm{P} 2 / \mathrm{P} 1 ;$

\%Removed because it is already receiving corrected compressor flow $\% \mathrm{~m} \_d o t \_c o r r e c t e d=\mathrm{m} \_d o t * \operatorname{sqrt}(\mathrm{T} 1 / 545) /(\mathrm{P} 1 * 2.03625437 / 28.4)$;

N_corrected_c $=$ interp2(comp_x_speed, comp_y_speed, comp_z_speed, m_dot, $\boldsymbol{k}$ $\mathrm{PR}$, 'linear');

N_phy = N_corrected_c*sqrt (T1/545);

end \%End of function 
\% Chris Forster

\% Compressor Outlet Pressure

function $[\mathrm{P} 2]=$ compressor_pressure $\left(\mathrm{P} 1, \mathrm{~T} 1, \mathrm{~W} \_\mathrm{c}, \mathrm{m} \_\right.$dot, comp_data $)$

C_p $=0.24 ;$

$P 2=P 1 *\left(W \_C^{*} \text { comp_data } / m \_d o t / C \_p / T 1+1\right)^{\wedge}(1 / \odot .283) ;$

end \%End of function 
\% Chris Forster

\% This function finds the power required from the compressor.

\%comp_data $(:, 1)=$ Corrected Speed

\%comp_data $(:, 2)=$ Corrected mass flow rate data

\%comp_data $(:, 3)=$ Pressure Ratio

function $\left[\right.$ power_c] = compressor_power $\left(\mathrm{P} 1, \mathrm{P} 2, \mathrm{~T} 1, \mathrm{~m} \_\right.$dot, eta_c $)$

$\mathrm{PR}=\mathrm{P} 2 / \mathrm{P} 1 ;$

power_c $=m_{-}$dot ${ }^{*} \odot .241^{*} T 1 /$ eta_c* $\left(P R^{\wedge} \odot .285-1\right) * \odot .02358 ; \quad$ \%Units are ink HP

end \%End of function 
\% Chris Forster

$\%$ This function finds the power required from the compressor.

\%comp_data $(:, 1)=$ Corrected Speed

\%comp_data $(:, 2)=$ Corrected mass flow rate data

\%comp_data $(:, 3)=$ Pressure Ratio

function $\left[\mathrm{m} \_\right.$dot_phy $]=$compressor_flow_phy $\left(\mathrm{P} 1, \mathrm{~T} 1, \mathrm{~m} \_\right.$dot_c $)$

m_dot_phy $=$ m_dot_c* $(P 1 * 2.03625437) / 28.4 / \operatorname{sqrt}(T 1 / 545) ; \quad$ \%Units ink $1 \mathrm{bm} / \mathrm{min}$

end \%End of function 
\% Chris Forster

\% The script runs the script to load the compressor data files and makes

\% uniform grids from sparse testing data.

comp_data_loader

\%comp_data_eta $(:, 1)=$ Corrected mass flow rate data

\%comp_data_eta $(:, 2)=$ Pressure Ratio

\%comp_data_eta $(:, 3)=$ Compressor Efficiency

acm.comp_x_eta $=\min ($ acm.comp_data.etafile $(:, 1)): 0.05: \max (\operatorname{acm} . \boldsymbol{k}$ comp_data.etafile $(:, 1))$;

acm.comp_y_eta $=\min ($ acm $\cdot$ comp_data.etafile $(:, 2)): 0.01: \max (\operatorname{acm} \cdot \boldsymbol{k}$

comp_data.etafile $(:, 2))$;

acm.comp_y_eta = acm. comp_y_eta';

acm.comp_z_eta $=\operatorname{griddata}(\operatorname{acm}$. comp_data.etafile $(:, 1)$, acm.comp_data.k etafile $(:, 2)$,acm.comp_data.etafile $(:, 3)$,acm.comp_x_eta,acm.k

comp_y_eta, 'cubic');

\%comp_data_speed $(:, 1)=$ Corrected Speed

\%comp_data_speed $(:, 2)=$ Corrected mass flow rate data

\%comp_data_speed $(:, 3)=$ Pressure Ratio

acm.comp_x_speed $=\min ($ acm.comp_data. $\operatorname{speedfile}(:, 2)): 0.01: \max (\operatorname{acm} . \boldsymbol{k}$ comp_data.speedfile $(:, 2))$;

acm.comp_y_speed $=\min ($ acm.comp_data. speedfile $(:, 3)): 0.01: \max (\operatorname{acm} . \mathbf{k}$ comp_data.speedfile $(:, 3))$;

acm. comp_y_speed $=$ acm . comp_y_speed ' ;

acm.comp_z_speed $=\operatorname{griddata}\left(\operatorname{acm} \cdot \operatorname{comp} \_d a t a \cdot \operatorname{speedfile}(:, 2)\right.$, acm. $\boldsymbol{k}$

comp_data.speedfile $(:, 3)$,acm.comp_data.speedfile $(:, 1)$, acm. $\boldsymbol{k}$

comp_x_speed,acm.comp_y_speed, 'cubic') ;

clc; 
\%Chris Forster

\%This file will be run from within the simulation (automatically...)

\% This script loads the two compressor data files (tab-delimitedk format)

acm.comp_data.speedfile = load('comp_data_speed.txt'); acm.comp_data.etafile = load('comp_data_eta.txt') ; 
\% Chris Forster

\% This function finds the compressor efficiency from the compressork data matrix.

\%comp_data $(:, 1)=$ Corrected Speed

\%comp_data $(:, 2)=$ Corrected mass flow rate data

\%comp_data $(:, 3)=$ Pressure Ratio

function[eta_c] = compressor_efficiency $\left(\mathrm{P} 1, \mathrm{P} 2, \mathrm{~T} 1, \mathrm{~m} \_\right.$dot, $\mathrm{comp} \_\mathrm{x} \_$eta, $k$ comp_y_eta,comp_z_eta)

$\mathrm{PR}=\mathrm{P} 2 / \mathrm{P} 1 ;$

eta_c $=$ interp2(comp_x_eta, comp_y_eta,comp_z_eta,m_dot,PR, 'linear') ;

end \%End of function 
\% Chris Forster

\% Turbine Mass Flow Rate

function[m_dot_t_corrected] = turbine_massflow_corrected $(P 1, T 1, k$ m_dot_t)

$m \_d o t \_t \_c o r r e c t e d=m \_d o t \_t * \operatorname{sqrt}(T 1 / 519) /(P 1 * 2.03625437 / 29.92)$;

end \%End of function 
Appendix J - ACM Performance Prediction Code in EES 
// Chris Forster

// 10/1/2009

// ACM Performance Prediction

// Functions Section

Function T_DAR(T_DB,g_tot,P_amb)

g_sat = humrat(airh2o,T=T_DB, $P=P$ _amb, $R=1$ )

g_ent = g_tot - g_sat

If(g_ent $>0)$ Then

If $\left(\left(T \_D B-459.67\right)>=32\right)$ Then

$A:=\left(\left(0.24+0.4332^{*} g \text { sat+g_ent }\right)^{\star}\left(T \_D B-459.67\right)-1093^{\star} g\right.$ ent $) /\left(0.24+0.4332^{\star}\right.$ g_tot $)$

Else

$A:=\left(\left(0.24+0.4332^{\star} g \_s a t+0.5^{\star} g \_e n t\right)^{*}\left(T \_D B-459.67\right)-1221^{*} g \_e n t\right) /\left(0.24+0.4332^{*} g\right.$ tot $)$

Endlf

Else

A:=(T_DB-459.67)

Endlf

T_DAR:=A+459.67

End

g_tot = humrat(airh2o, T=T_amb, P=P_amb, R=R_amb)

// End Functions Section

fluid $\$=$ 'air'

// debugging stuff

$\left\{m \_d o t=10\right\}$

$\left\{N \_\right.$phy $\left.=95000\right\}$

$\left\{W_{-} t \_c=6\right\}$

// end debugging stuff

// Ambient Conditions

T_amb $=531[\mathrm{R}]$

P_amb $=14.69$ [psia]

R_amb $=0.40$

// ACM Inlet Conditions

T_1c $=526[\mathrm{R}]$

$\left\{P \_1 c=25.5[\right.$ psia $\left.]\right\}$

$\left\{P R \_A C M=1.5\right\}$

$\mathrm{PR} \_\mathrm{ACM}=\mathrm{P} \_1 \mathrm{c} / \mathrm{P} \_2 \mathrm{t}$

// Heat Exchanger Data

E_htxr $=0.83$

dp_htxr = 0.3 [psi]

gamma $=1.4$

$\mathrm{n}=($ gamma-1)/gamma

// Compressor Work Calculation

PR_c $=$ P_2c/P_1c

W_dot_c $=m$ _dot ${ }^{\star} C P\left(\text { fluid } \$, T=T \_1 c\right)^{*} T$ 1c/eta_c* $\left(P R \_c^{\wedge} n-1\right) * 0.02358$ 
T_2c $=T \_1 c+T \_1 c^{*}\left(P R \_c^{\wedge} n-1\right) / e t a \_c$

// HTXR Calculations

P_1t = P_2c - dp_htxr

T_1t $=$ T_2c - E_htxr* $\left(T \_2 c-T \_a m b\right)$

// Turbine Work Calculation

PR_t $=$ P_1t $/ P \_2 t$

$P \_2 t=14.69$

W_dot_t $=$ m_dot ${ }^{\star} C P(\text { fluid } \$, T=T \text { Tt })^{\star} T$ _1 ${ }^{\star}$ eta_t ${ }^{\star}\left(P R \_t^{\wedge} n-1\right) / P R \_t^{\wedge} n^{\star} 0.02358$

T_2t $=$ T_1t - W_dot_t/0.02358/m_dot/CP(fluid $\left.\$, T=T \_1 t\right)$

// Compressor-Turbine Relationship

W_dot_c $=$ W_dot_t

// Compressor and Turbine Map Interpolation

W_c_c $=$ m_dot*sqrt $\left(T \_1 c / 545\right) /\left(P \_1 c^{\star} 2.03625437 / 28.4\right)$

eta_c = interpolate2D('c_data', 'W_c', 'PR_c', 'Eta_c', W_c $=$ W_c_c, PR_c $=$ PR_c)

N_c_c = interpolate2D('N_c', 'W_c', 'PR_c', 'N_c', W_c = W_c_c, PR_c = PR_c)

N_c_c $=$ N_phy/sqrt $\left(T \_1 c / 545\right)$

N_t_c $=$ N_phy/sqrt $\left(T \_1 t / 519\right)$

W_t_c $=$ m_dot*sqrt $\left(T \_1 t / 519\right) /\left(P \_1 t^{\star} 2.03625437 / 29.92\right)$

eta_t $=$ interpolate2D('t_data', 'W_t', 'PR_t', 'Eta_t', W_t $=$ W_t_c, PR_t $=$ PR_t)

W_t_c = interpolate2D('t_data', 'N_t', 'PR_t', 'W_t', N_t = N_t_c, PR_t = PR_t)

// Capacity Calculations

Q_dot_dry $=m$ _dot ${ }^{\star} \mathrm{CP}($ fluid $\$, T=T$ _amb $){ }^{\star}\left(T \text { _amb }-\mathrm{T} \_2 \mathrm{t}\right)^{\star} 60 / 12000$

T_2t_DAR $=$ T_DAR $\left(T \_2 t, g \_t o t, P \_a m b\right)$

Q_dot_DAR $=m$ _dot* $\mathrm{CP}(\text { fluid } \$, T=T \text { _amb })^{\star}(T \text { _amb }-T \text { _t__DAR })^{\star} 60 / 12000$

// Primary/Source Compressor Calculations

PR_ce $=$ P_1c/P_amb

eta_ce $=0.70$

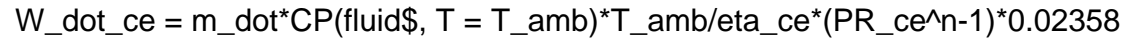

// COP Calculations

COP_dry = Q_dot_dry*4.71617735/W_dot_ce

COP_DAR $=$ Q_dot_DAR*4.71617735/W_dot_ce

\section{SOLUTION}

Unit Settings: [R]/[psia]/[lbm]/[degrees]

\section{(Table 1, Run 20)}

COPDAR $=0.6145$

$$
\text { COPdry }=0.4299
$$

$\mathrm{dphtxr}=0.3$ [psi]

$\eta^{c}=0.6317$

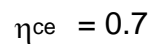

$\eta^{\mathrm{t}}=0.5873$

$E_{h t x r}=0.83$

fluid\$ = 'air'

$\gamma=1.4$

gtot $=0.006505$

$\dot{\mathrm{m}}=14.16$

$\mathrm{n}=0.2857$

$\mathrm{N}_{\mathrm{c}, \mathrm{c}}=109524$

Nphy $=107598$

$\mathrm{Nt}, \mathrm{c}=105396$

PRACM $=1.7$

$\mathrm{PR}_{\mathrm{c}}=1.292$

$\mathrm{PR}_{\text {ce }}=1.7$

$\mathrm{PRt}_{\mathrm{t}}=2.177$ 
$\mathrm{P}_{1 \mathrm{t}}=31.98$

$\mathrm{P}_{2 \mathrm{t}}=14.69$

Q்DAR $=1.296$

$\mathrm{R}_{\mathrm{amb}}=0.4$

$\mathrm{T}_{1 \mathrm{t}}=540.9$

$\mathrm{T}_{2 \mathrm{t}}=477.6$

$\mathrm{T}_{\mathrm{amb}}=531[\mathrm{R}]$

$\dot{\mathrm{W}}_{\mathrm{c}}=5.072[\mathrm{Btu} / \mathrm{lbm}]$

$\dot{\mathrm{W}}_{\mathrm{t}}=5.072$

No unit problems were detected.

EES suggested units (shown in purple) for T_2c W_dot_c .

$$
\begin{aligned}
& \mathrm{P}_{2 c}=32.28 \\
& \mathrm{P}_{a m b}=14.69 \text { [psia] } \\
& \dot{\mathrm{Q}}_{\mathrm{dry}}=0.9068 \\
& \mathrm{~T}_{1 \mathrm{c}}=526[\mathrm{R}] \\
& \mathrm{T}_{2 \mathrm{c}}=589.3[\mathrm{R}] \\
& \mathrm{T}_{2 \mathrm{t}, \mathrm{DAR}}=454.7 \\
& \mathrm{~W}_{\mathrm{c}, \mathrm{c}}=7.769 \\
& \dot{\mathrm{W}}_{\mathrm{ce}}=9.947 \\
& \mathrm{~W}_{\mathrm{t}, \mathrm{c}}=6.642
\end{aligned}
$$


Appendix K - ACM Single-Point Performance Comparison in EES 


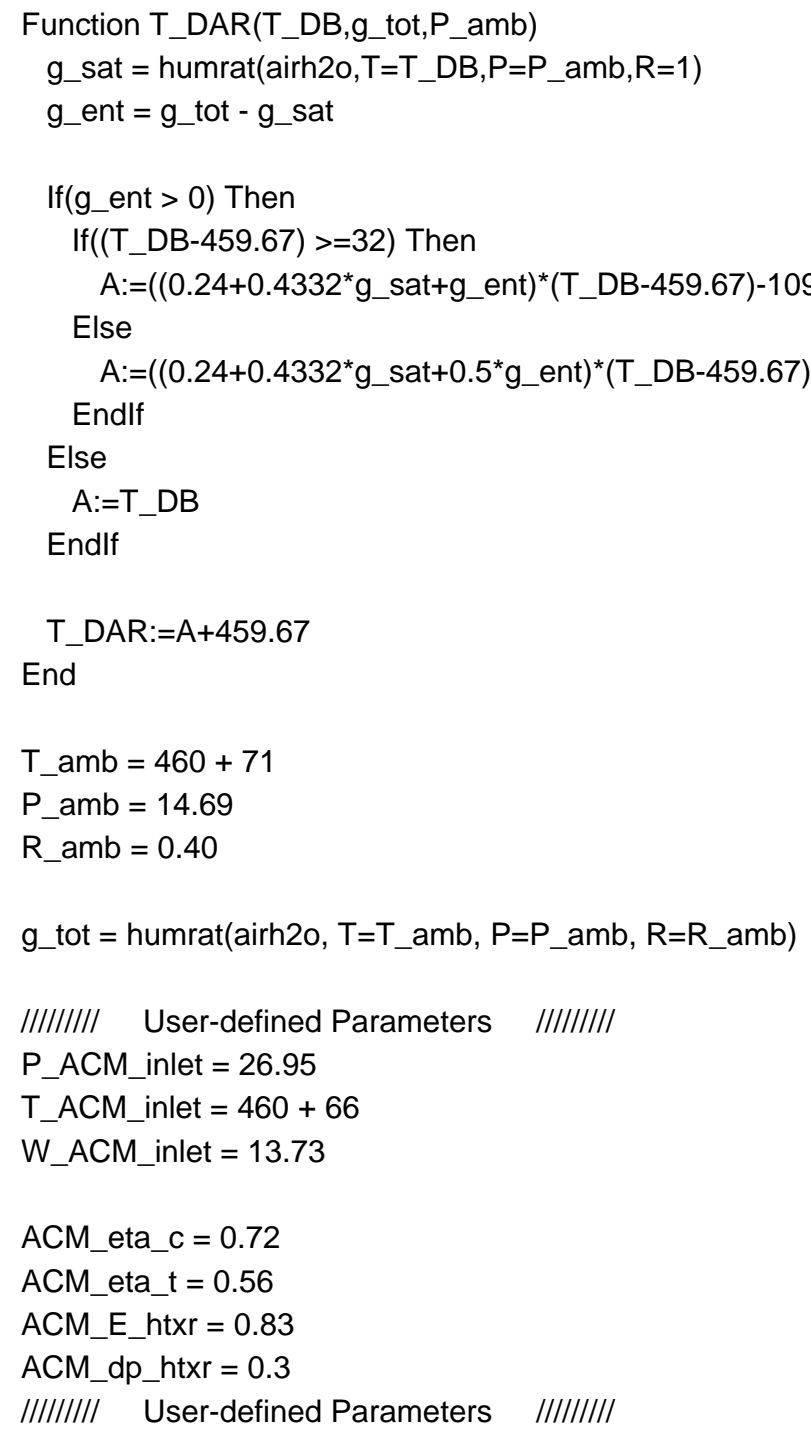

"Engine Parameters"

eta_c1 $=0.7$

W_bleed $=$ W_ACM_inlet

boost $=$ P_ACM_inlet $-\mathrm{P} \_$amb $\quad$ \{Comment this out for parametric table operations... $\}$

E_htxr1 = 1

dp_htxr1 =0

"ACM Parameters"

eta_c2 = ACM_eta_c

eta_t2 = ACM_eta_t

E_htxr2 = ACM_E_htxr

dp_htxr2 = ACM_dp_htxr

"Primary Compressor Power"

power_c1 $=$ W_bleed*0.24*T_1c1*(PR_c1^0.285-1)/eta_c1*0.02358

"Power Balance"

power_c2 $=$ W_bleed*0.24*T_1c2* $\left(P R \_c 2^{\wedge} 0.285-1\right) /$ eta_c2*0.02358

power_t2 $=$ W_bleed ${ }^{\star} 0.24^{*} T \_1 t 2^{\star}\left(P R \_t 2^{\wedge} 0.285-1\right) / P R \_t 2^{\wedge} 0.285^{\star}$ eta_t2 ${ }^{\star} 0.02358$ 
power_c2 = power_t2

"T_1c2* $\left(P R \_c 2^{\wedge} 0.285-1\right) / e t a \_c 2=T \_1 t 2^{*}\left(P R \_t 2^{\wedge} 0.285-1\right) / P R \_t 2^{\wedge} 0.285^{\star} e t a \_t 2 "$

"Pressure Ratios"

PR_c1 $=\left(P \_a m b+b o o s t\right) / P \_a m b$

$P R \_c 2=P \_2 c 2 / P \_1 c 2$

PR_t2 = P_1t2/P_2t2

$P R \_A C M=P \_1 c 2 / P \_2 t 2$

$P \_2 c 1=P \_a m b+b o o s t$

$P \_1 c 2=P \_A C M \_$inlet

$P \_1 t 2=P \_2 c 2-d p \_h t \times r 2$

$\{$ User-defined\}

P_2t2 $=P \_a m b$

"Temperatures"

T_1c1 = T_amb

T_2c1 = T_1c1 + T_1c1*(PR_c1^0.285-1)/eta_c1

T_1htxr1 $=$ T_2c1

T_2htxr1 = T_1htxr1 - E_htxr1*(T_1htxr1-T_amb)

T_1c2 = T_2htxr1

$\mathrm{T} \_2 \mathrm{c} 2=\mathrm{T} \_\mathrm{ACM}$ inlet $\quad\{$ User-defined $\}$

T_1htxr2 = T_2c2

T_2htxr2 = T_1htxr2 - E_htxr2*(T_1htxr2-T_amb)

T_1t2 = T_2htxr2

T_2t2 = T_1t2 - power_t2/0.02358/W_bleed $/ 0.24$

T_2t2_DAR $=$ T_DAR(T_2t2,g_tot,P_amb)

Q_DAR $=$ W_bleed ${ }^{*} 0.241 *\left(T \_a m b-T \_2 t 2\right.$ _DAR $) * 60 / 12000$

COP_DAR $=$ Q_DAR/(power_c1/0.02358)/60*12000

\{"Uncertainty Analysis Section"

"Measurement Uncertainties"

$T \_1 c 2 \_u=1.7$

$T \_2 c 2 \_u=1.7$

P_1c2_u $=0.02$

$P \_2 c 2 \_u=0.02$

"Compressor Efficiency"

\} 
Appendix L - ACM Component Efficiency Variation Simulation 


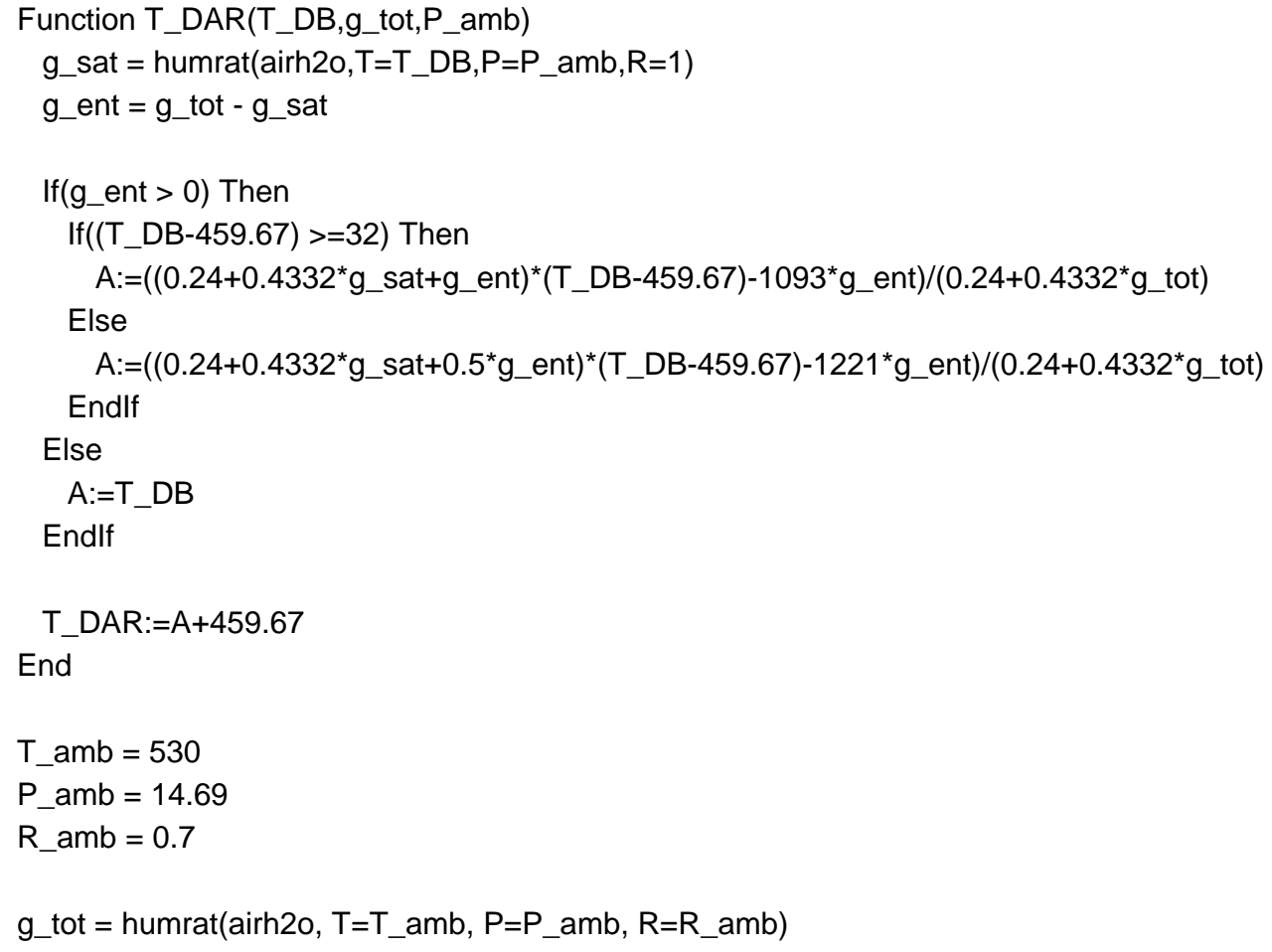


P_1c2 $=$ P_2c1 $-d p \_h t x r 1$
P_1t2 $=P \_2 c 2-d p \_h t x r 2$
$P \_2 t 2=P \_a m b$

"Temperatures"

T_1c1 = T_amb

T_2c1 $=$ T_1c1 + T_1c1*(PR_c1^0.285-1)/eta_c1

T_1htxr1 = T_2c1

T_2htxr1 = T_1htxr1 - E_htxr1*(T_1htxr1-T_amb)

T_1c2 = T_2htxr1

T_2c2 $=$ T_1c2 + T_1c2* $\left(P R \_c 2^{\wedge} 0.285-1\right) /$ eta_c2

T_1htxr2 = T_2c2

T_2htxr2 = T_1htxr2 - E_htxr2*(T_1htxr2-T_amb)

T_1t2 = T_2htxr2

T_2t2 = T_1t2 - power_t2/0.02358/W_bleed $/ 0.24$

T_2t2_DAR $=$ T_DAR(T_2t2,g_tot,P_amb)

Q_DAR $=$ W_bleed ${ }^{*} 0.241 *\left(T \_a m b-T \_2 t 2 \_D A R\right) * 60 / 12000$

COP_DAR $=$ Q_DAR/(power_c1/0.02358)/60*12000

\{"Uncertainty Analysis Section"

"Measurement Uncertainties"

$T \_1 c 2 \_u=1.7$

$T \_2 c 2 \_u=1.7$

P_1c2_u $=0.02$

P_2c2_u $=0.02$

"Compressor Efficiency"

\} 
Appendix M - ACM Post-processing Code in Matlab 
\% Chris Forster

$\% 5 / 2 / 2009$

clear;

clc;

close all;

\%\% Ambient Test Conditions

Ambient_Pressure $=14.69$;

\% Ambient_Temperature $=83.5 ; \quad$ \%Deg. $\mathrm{F}$

Ambient_Temperature $=71 ; \quad$ \%Deg. $\mathrm{F}$

Rel_hum $=0.40$;

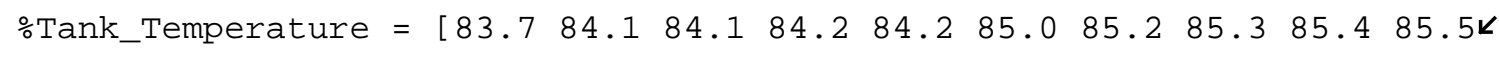
86.1 71]; \%Deg F

Tank_Temperature $=65^{*}$ ones $(1,7) ; \quad$ \%Deg $\mathrm{F}$

$\%$ Column Assignments - Data

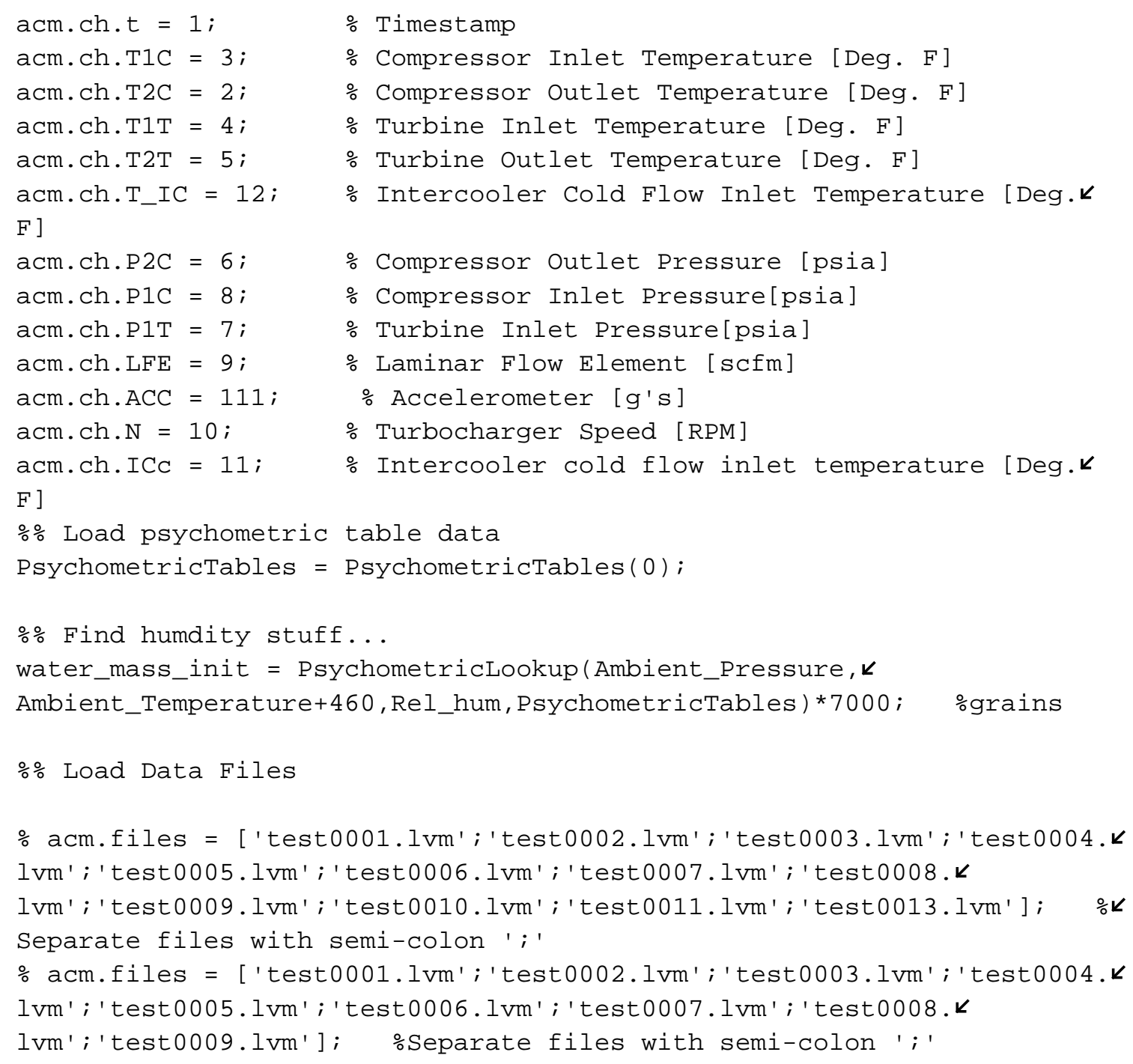




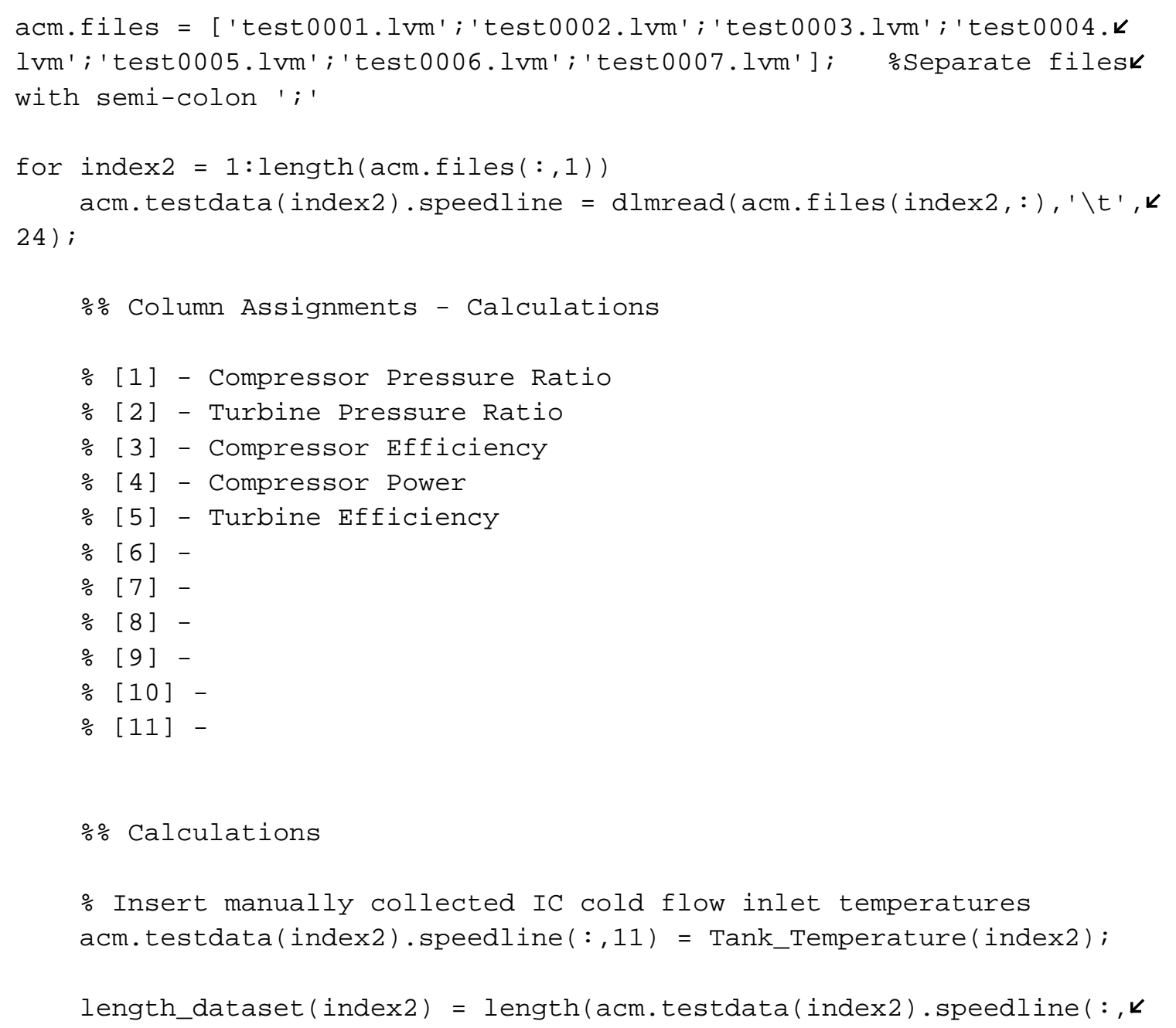




$$
\text { ch. P1C); }
$$

end

$\operatorname{acm} \cdot \operatorname{avg}(1$, index2 $)=\operatorname{mean}(\operatorname{acm} \cdot \operatorname{calcs}($ index2 $) \cdot \operatorname{speedline}(:, 1)) ;$

\% Turbine Pressure Ratio

for index $=1:$ length_dataset $($ index2)

acm.calcs (index2). speedline (index, 2) $=\operatorname{acm} \cdot$ testdata (index 2$) \cdot \boldsymbol{k}$ speedline(index, acm.ch.P1T)/Ambient_Pressure;

end

$\operatorname{acm} \cdot \operatorname{avg}(2$, index 2$)=\operatorname{mean}(\operatorname{acm} \cdot \operatorname{calcs}($ index 2$) \cdot \operatorname{speedline}(:, 2))$;

\% Compressor Efficiency

for index $=1$ : length_dataset $($ index2)

acm.calcs (index2). speedline (index, 3$)=($ acm.testdata (index 2$) \cdot k$ speedline (index, acm.ch. T1C) +460$) *(a c m . c a l c s($ index 2$)$. speedline (index, 1)k $\wedge \odot .285-1) /(($ acm . testdata (index 2$)$. speedline (index, acm. ch. T2C $)+460)-k$ (acm. testdata(index2). speedline (index, acm.ch.T1C)+460));

end

$\operatorname{acm} \cdot \operatorname{avg}(3$, index2 $)=$ mean $(\operatorname{acm} \cdot \operatorname{calcs}($ index 2$) \cdot \operatorname{speedline}(:, 3))$;

$\%$ Mass Flowrate

for index = 1:length_dataset (index2)

acm.calcs (index2). speedline (index, 4) = acm.testdata (index2). $\boldsymbol{k}$ speedline (index, acm.ch. LFE) *acm. testdata(index 2 ). speedline (index, acm. $\boldsymbol{k}$ ch.P1C)/(acm . testdata (index2) . speedline (index, acm. ch. T1C) +460$) * 2 . k$ $699504268^{*}$ viscosity_correction(acm.testdata(index 2 ). speedline(index, $\boldsymbol{k}$ acm.ch.T1C));

end

$\operatorname{acm} \cdot \operatorname{avg}(4$, index2 $)=$ mean $(\operatorname{acm} \cdot \operatorname{calcs}($ index 2$) \cdot \operatorname{speedline}(:, 4))$;

\% Compressor Power

for index $=1$ : length_dataset $($ index 2$)$ acm.calcs (index2). speedline (index, 5) = acm.calcs (index 2$) \cdot \boldsymbol{k}$ speedline (index, 4)* $\odot .241 *$ (acm. testdata (index2). speedline (index, acm.ch.k T1C) +460 ) /acm . calcs (index2) . speedline (index, 3$)^{*}$ (acm . calcs (index2) . $k$ speedline $\left.(\text { index }, 1)^{\wedge} \odot .285-1\right) * \odot .02358$;

end

$\operatorname{acm} \cdot \operatorname{avg}(5$, index2 $)=$ mean $(\operatorname{acm} \cdot \operatorname{calcs}($ index 2$) \cdot \operatorname{speedline}(:, 5))$;

\% Turbine Efficiency (WITH BEARING LOSSES)

for index $=1$ : length_dataset $($ index 2$)$ acm.calcs (index2). speedline $($ index, 6$)=\operatorname{acm} \cdot \operatorname{calcs}($ index 2$) \cdot \boldsymbol{k}$ speedline (index, 5)/acm. calcs (index2). speedline (index, 4)/0.241/(acm. $\boldsymbol{k}$ testdata (index2). speedline(index, acm.ch.T1T) +460$) /($ acm. calcs (index2) . $k$ 
speedline $\left.(\text { index }, 2)^{\wedge} \odot .285-1\right)^{\star}$ acm. calcs (index2). speedline (index, 2$)^{\wedge} \odot . \kappa$ 285/0.02358;

end

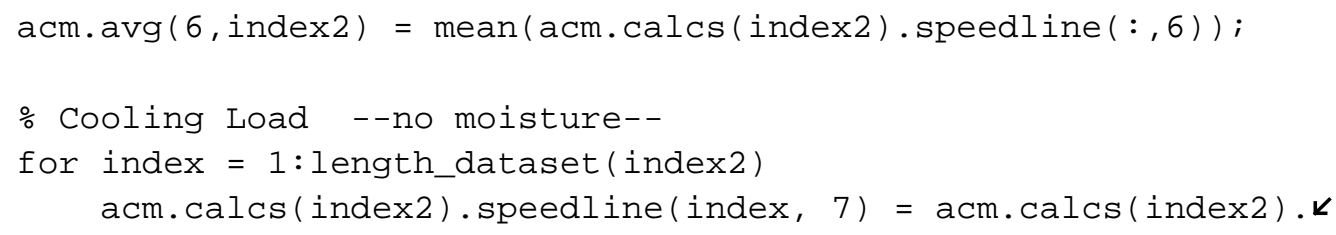

end 


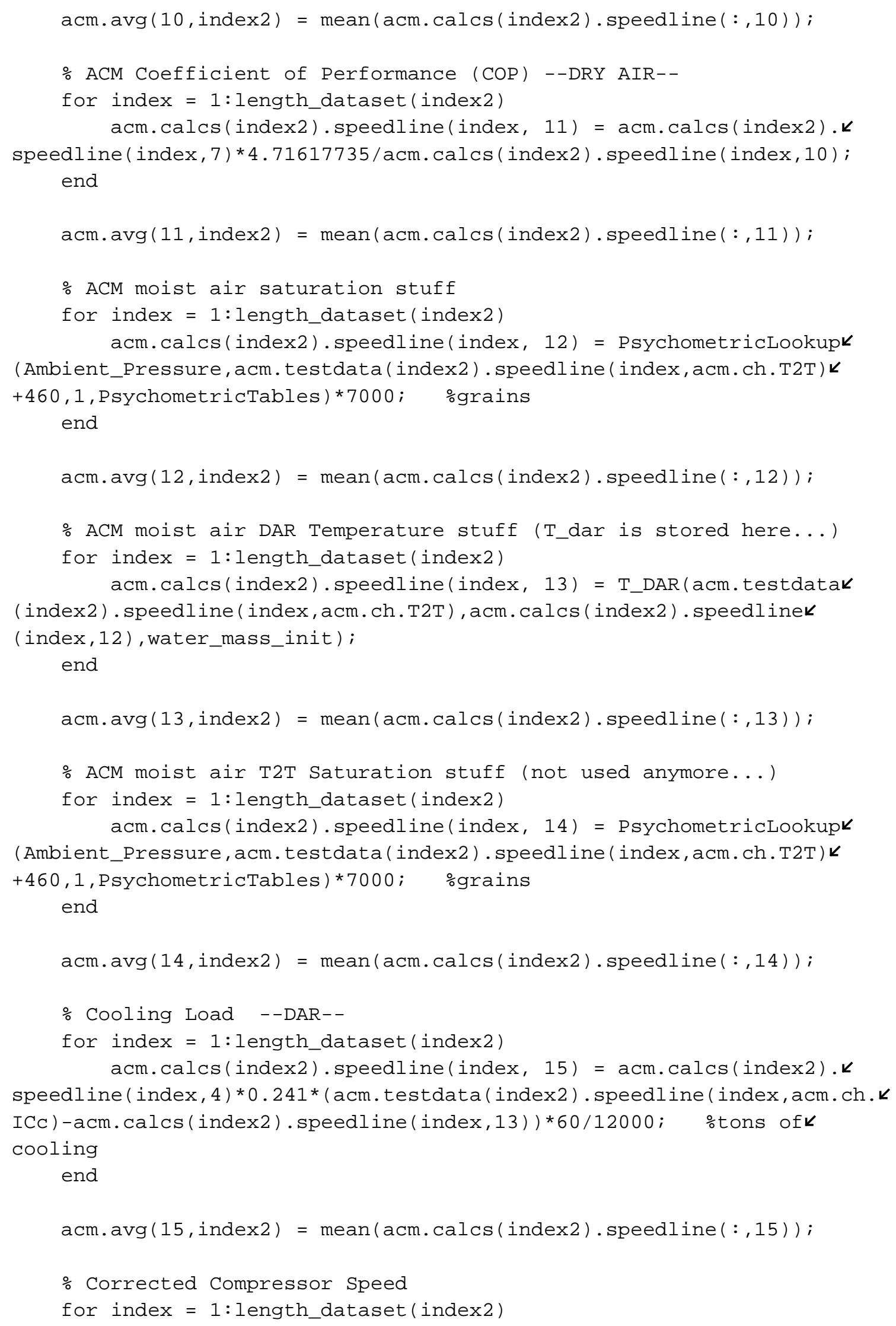


acm.calcs (index2).speedline (index, 16) = acm.testdata(index2). $\boldsymbol{k}$ speedline (index, acm.ch.N)/sqrt ( (acm.testdata(index2). speedline(index, $\boldsymbol{k}$ acm.ch. T1C) +460$) / 545)$;

end

$\operatorname{acm} \cdot \operatorname{avg}(16$, index2 $)=$ mean $(\operatorname{acm} \cdot \operatorname{calcs}($ index2 $) \cdot \operatorname{speedline}(:, 16)) ;$

\% ACM Coefficient of Performance (COP) --DAR--

for index = 1:length_dataset $($ index 2$)$

acm.calcs (index2). speedline (index, 17) $=$ acm.calcs (index2).k

speedline (index, 15)*4.71617735/acm. calcs (index2). speedline (index, 10);

end

$\operatorname{acm} \cdot \operatorname{avg}(17$, index2 $)=$ mean $(\operatorname{acm} \cdot \operatorname{calcs}($ index2 $) \cdot$ speedline $(:, 17))$;

\% ACM IC Effectiveness Estimate (without cold flow inlet temp, $\boldsymbol{k}$ assumed to be ambient...)

$\%$ for index $=1:$ length_dataset $($ index 2$)$

$\% \quad$ acm.calcs (index2). speedline (index, 18) $=($ acm.testdatak (index2).speedline (index, acm.ch.T2C)-acm. testdata (index2) . speedlinek (index, acm.ch.T1T) )/(acm. testdata (index2). speedline (index, acm. ch. T2C) - $k$ acm. testdata(index2). speedline(index, acm.ch.T1C));

$\% \quad$ end

\% ACM IC Effectiveness Estimate Using Measured IC Cold Flow Inletk Temperatures

for index $=1:$ length_dataset $($ index2)

acm.calcs (index2). speedline (index, 18) = (acm.testdatak

(index2). speedline (index, acm.ch.T2C)-acm. testdata(index2).speedlinek

(index, acm.ch.T1T) )/(acm. testdata (index2). speedline (index, acm.ch.T2C) - $\boldsymbol{k}$ acm. testdata(index2). speedline(index, acm.ch.ICc));

end

$\operatorname{acm} \cdot \operatorname{avg}(18$, index2 $)=\operatorname{mean}(\operatorname{acm} \cdot \operatorname{calcs}($ index2 $) \cdot \operatorname{speedline}(:, 18))$;

\% Corrected Turbine Speed

for index $=1$ : length_dataset $($ index2)

acm.calcs(index2). speedline(index, 19) $=$ acm.testdata(index2). $k$ speedline (index, acm.ch.N)/sqrt ( (acm. testdata(index 2$)$. speedline (index, $k$ acm.ch. T1T) +460$) / 519$ );

end

$\operatorname{acm} \cdot \operatorname{avg}(19$, index2 $)=\operatorname{mean}(\operatorname{acm} \cdot \operatorname{calcs}($ index2 $) \cdot \operatorname{speedline}(:, 19)) ;$

\% Turbine Efficiency (WITHOUT BEARING LOSSES)

for index $=1$ : length_dataset $($ index 2$)$

\%acm.calcs (index 2$)$. speedline (index, 20) $=(1-($ acm.calcsk

(index2) . speedline (index, 13) +460$) /($ acm . testdata (index2). speedlinek

$($ index, $\mathrm{acm}$. ch. T1T $)+460)) /(($ acm. calcs $($ index2 $)$. speedline $($ index, 2$) \wedge \odot .285-k$ 
1)/acm. calcs (index2). speedline (index, 2)^৫.285);

acm.calcs (index2). speedline (index, 20) $=(1-($ acm. testdatak

(index2). speedline (index, acm.ch.T2T) +460$) /($ acm . testdata (index 2$) \cdot k$ speedline (index, acm. ch. T1T) +460) )/( (acm. calcs (index2) . speedline (index, $k$ $\left.2)^{\wedge} \odot .285-1\right) /$ acm. calcs (index2). speedline (index, 2)^৩.285);

end

$\operatorname{acm} \cdot \operatorname{avg}(20$, index2 $)=$ mean $(\operatorname{acm} \cdot \operatorname{calcs}($ index2 $) \cdot \operatorname{speedline}(:, 20))$;

\% Overall Cycle Pressure Ratio

for index $=1:$ length_dataset $($ index 2$)$

$\mathrm{acm} . \mathrm{calcs}($ index 2$) \cdot$ speedline (index, 21) $=$ acm.testdata(index2).k speedline(index, acm.ch.P1C)/Ambient_Pressure;

end

$\operatorname{acm} \cdot \operatorname{avg}(21$, index2 $)=\operatorname{mean}(\operatorname{acm} \cdot \operatorname{calcs}($ index2 $) \cdot \operatorname{speedline}(:, 21)) ;$

\% Bearing Efficiency

for index = 1:length_dataset $($ index 2$)$

$\mathrm{acm} . \operatorname{calcs}($ index 2$)$. speedline (index, 22) $=1-(\mathrm{acm} \cdot \operatorname{calcs}($ index 2$) \cdot k$

speedline(index, 20)-acm.calcs(index2).speedline(index, 6));

end

acm. $\operatorname{avg}(22$, index2 $)=$ mean $(\operatorname{acm} \cdot \operatorname{calcs}($ index 2$) \cdot$ speedline $(:, 22))$;

\% Turbine Power (before bearing losses)

for index $=1$ : length_dataset (index2)

acm.calcs (index2). speedline (index, 23) $=$ acm.calcs (index2) $\cdot \boldsymbol{k}$

speedline (index, 4) * $0.241 *$ (acm.testdata(index 2$)$. speedline(index, $\boldsymbol{k}$ acm.ch.T1T) - acm. testdata (index2). speedline (index, acm.ch.T2T)) ${ }^{*} \kappa$

$\odot .02358$;

end

$\operatorname{acm} \cdot \operatorname{avg}(23$, index2 $)=\operatorname{mean}(\operatorname{acm} \cdot \operatorname{calcs}($ index2 $) \cdot \operatorname{speedline}(:, 23))$;

\% Bearing Efficiency (Recalculated using power)

for index $=1$ : length_dataset $($ index2)

acm.calcs (index2). speedline(index, 24) = 1 - (acm.calcsk

(index2).speedline(index, 23) - acm.calcs(index2).speedline(index, 5)) $k$ /acm.calcs (index2). speedline(index, 23);

end

$\operatorname{acm} \cdot \operatorname{avg}(24$, index2 $)=$ mean $(\operatorname{acm} \cdot \operatorname{calcs}($ index2 $) \cdot \operatorname{speedline}(:, 24))$;

\% Compressor Outlet Temperature vs. overall ACM PR

for index $=1$ : length_dataset $($ index 2$)$

acm.calcs (index2). speedline (index, 25) = acm.testdata (index2).k

speedline(index, 2);

end 
$\operatorname{acm} \cdot \operatorname{avg}(25$, index2 $)=\operatorname{mean}(\operatorname{acm} \cdot \operatorname{calcs}($ index 2$) \cdot \operatorname{speedline}(:, 25))$;

for index3 $=1: 10$

acm.avgcalc $($ index 3, index 2$)=\operatorname{mean}(\operatorname{acm}$. testdata $($ index 2$) \cdot \boldsymbol{k}$ speedline ( : , index 3$))$;

end

end

$\%$ Plots

\% Plot smoothing

datap = 100;

scatter_bit $=0 ; \quad \% \odot$ scatter off / 1 scatter on

for index $=1:$ length $(\operatorname{acm} \cdot \operatorname{avg}(:, 1))$

acm.xi(index, $:)=\min (\operatorname{acm} \cdot \operatorname{avg}($ index, $:)):(\max (\operatorname{acm} \cdot \operatorname{avg}($ index, $:))-\operatorname{mink}$ $(\operatorname{acm} . \operatorname{avg}($ index, : $)))$ /datap: $\max (\operatorname{acm} . \operatorname{avg}($ index, $:))$;

end

for index $=1:$ length $(\operatorname{acm} . \operatorname{avgcalc}(:, 1))$

acm.xicalc $($ index,$:)=\min (\operatorname{acm} . \operatorname{avgcalc}($ index, $:)):(\max (\operatorname{acm}$. avgcalck

(index, : ) ) - min (acm.avgcalc (index, : )))/datap: $\max (\operatorname{acm}$. avgcalc(index, : )) ;

end

\% Compressor Locus of Operating Points

figure(1)

$\operatorname{plot}(\operatorname{acm} . \operatorname{avg}(8,:), \operatorname{acm} . \operatorname{avg}(1,:))$

grid on

xlabel('Corrected Compressor Flow Rate [lbm/min]')

ylabel('Compressor Pressure Ratio [-]')

hold on

if scatter_bit $==1$

for index $=1:$ length $(\operatorname{acm}$. files $(:, 1))$ scatter (acm. calcs (index) . speedline $(:, 8), \operatorname{acm}$. calcs (index) . $k$

speedline $(:, 1))$

end

else

scatter $(\operatorname{acm} . \operatorname{avg}(8,:), \operatorname{acm} . \operatorname{avg}(1,:))$

end

\% Turbine Locus of Operating Points 


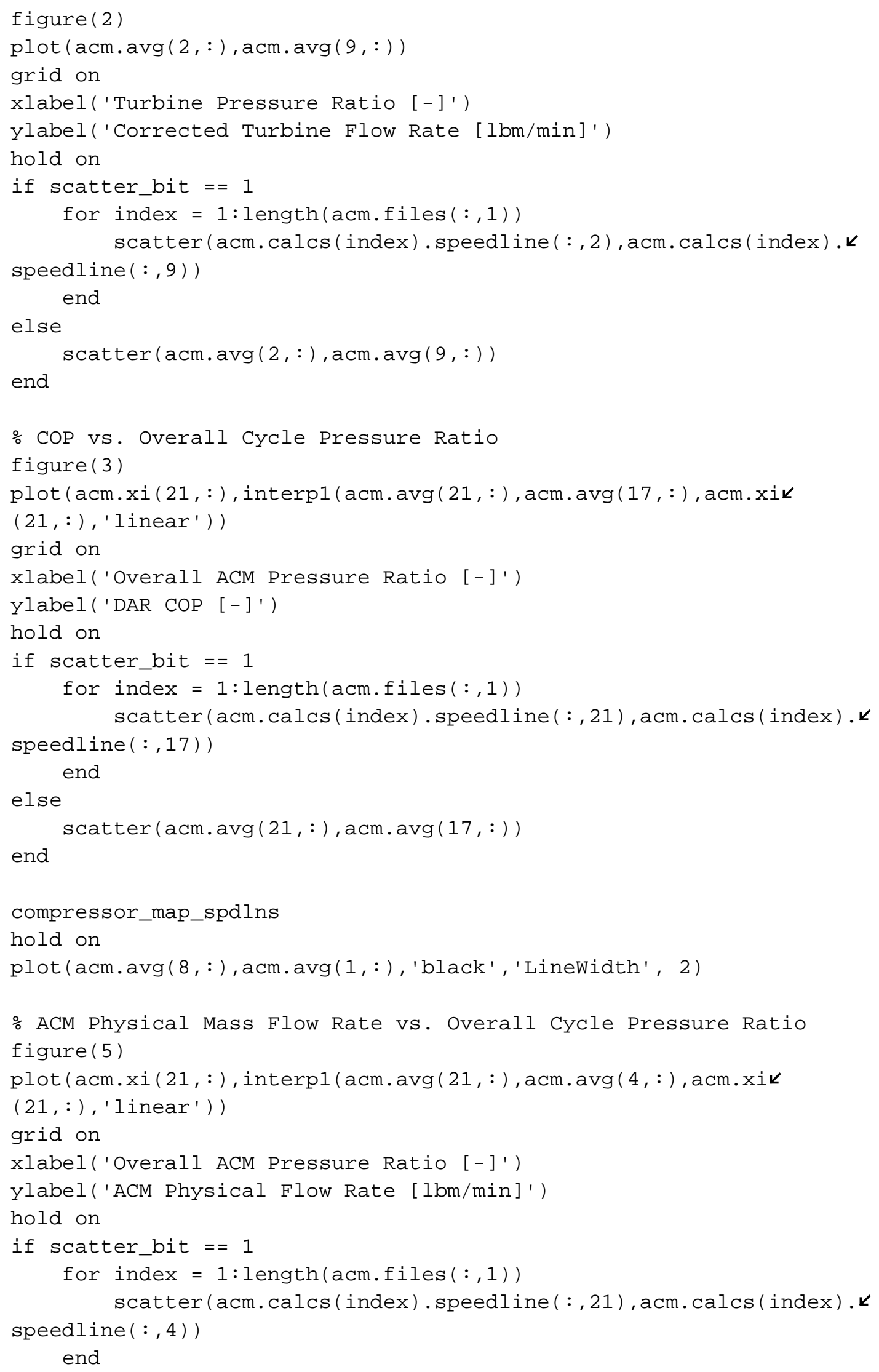




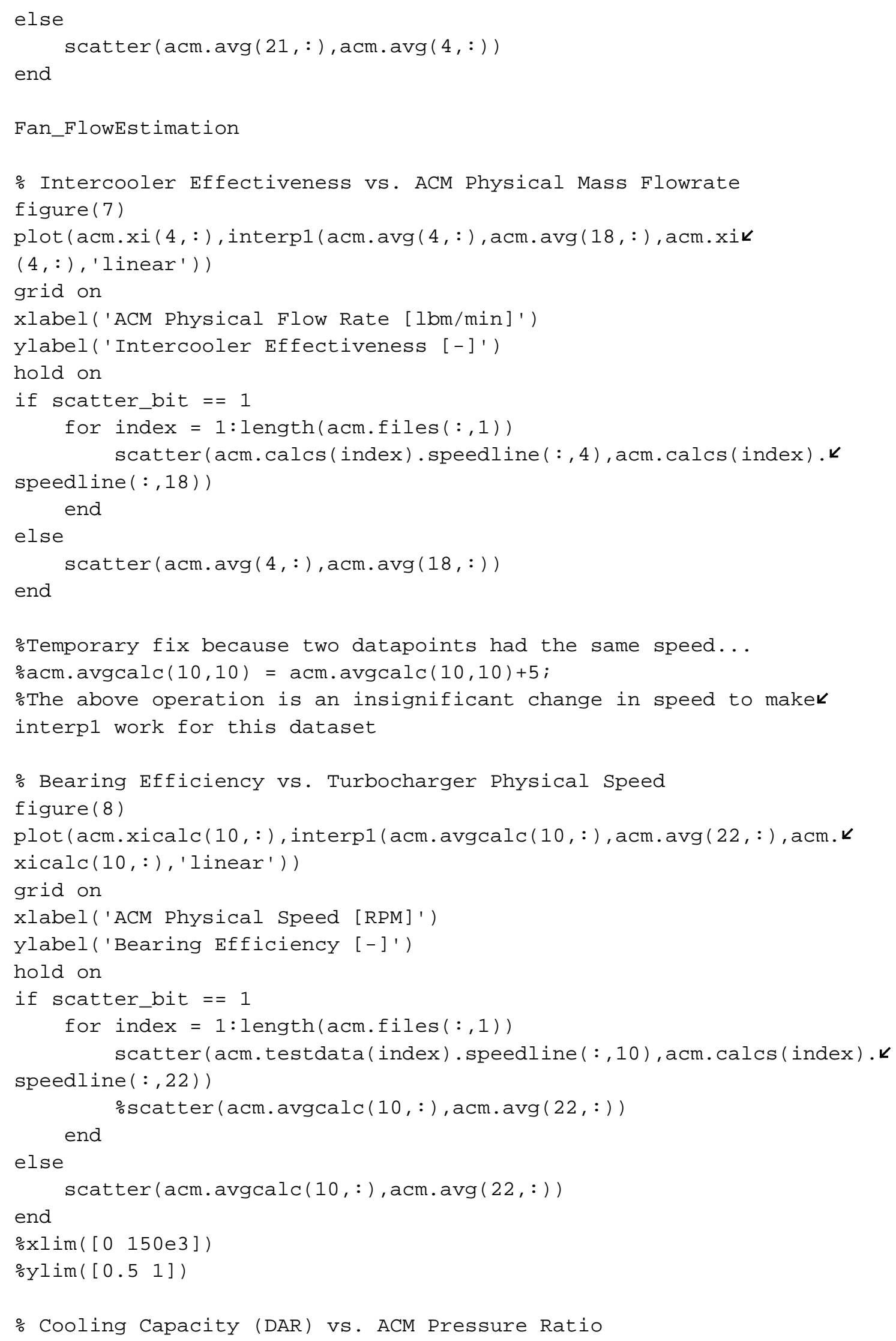




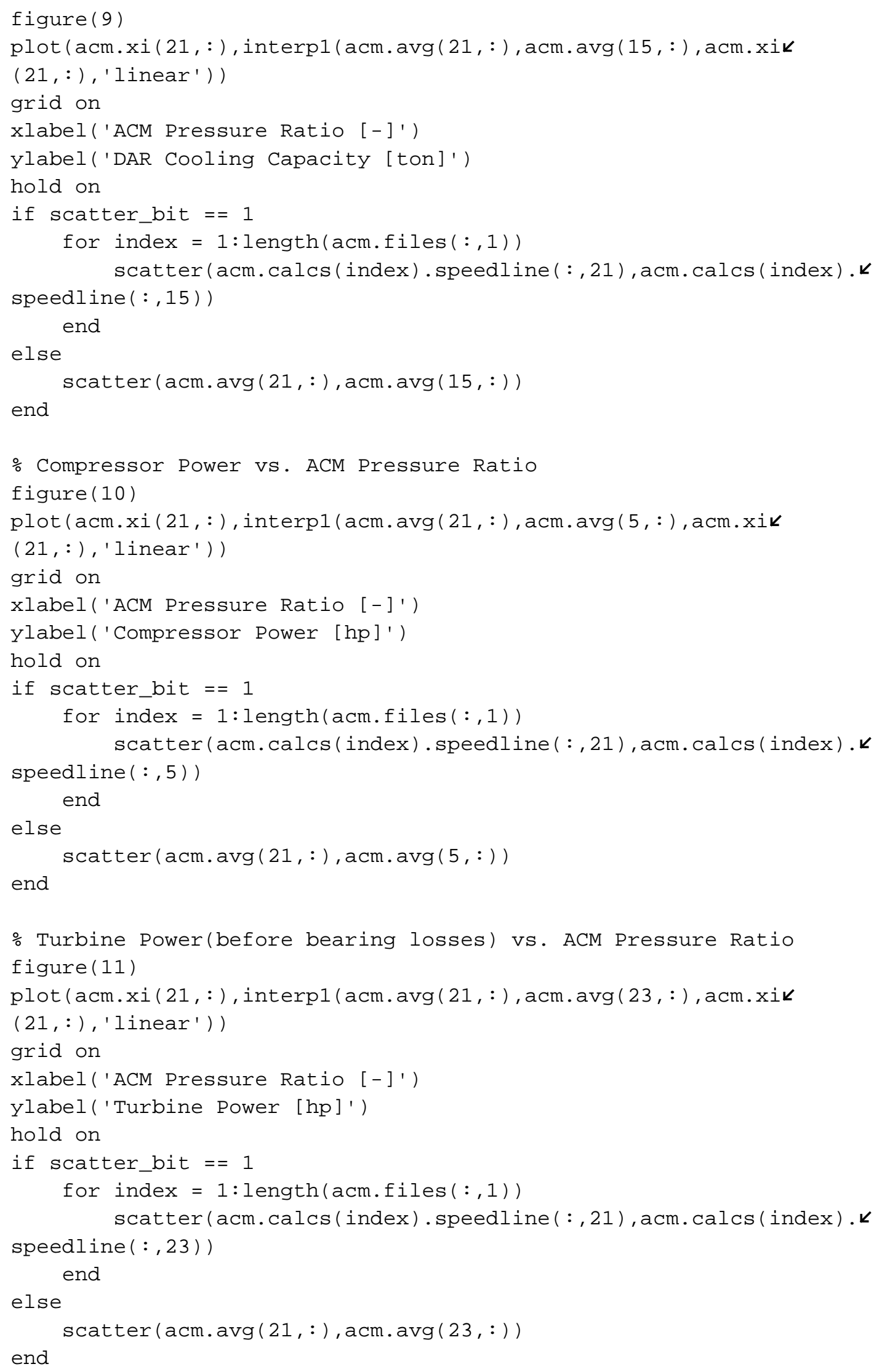




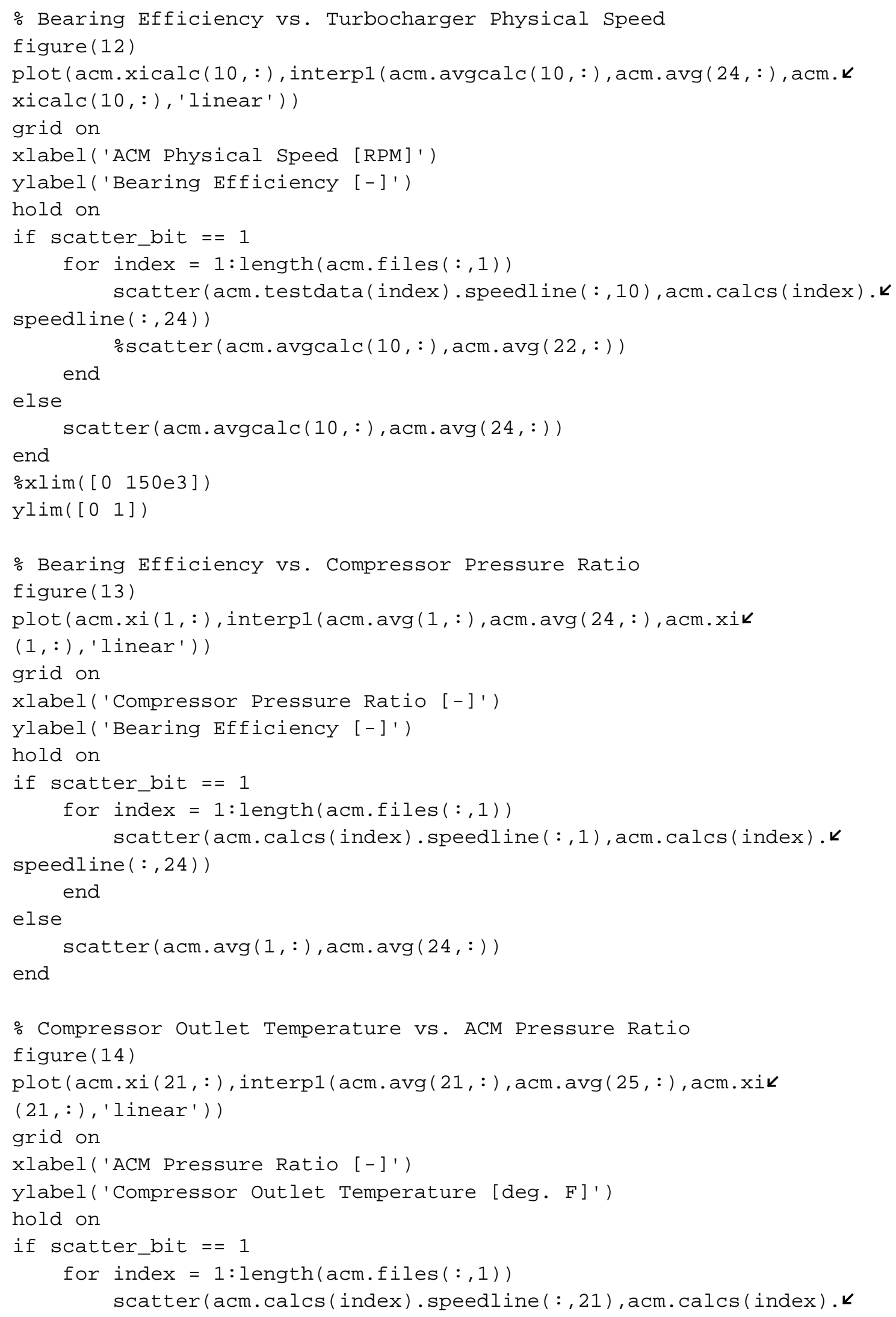




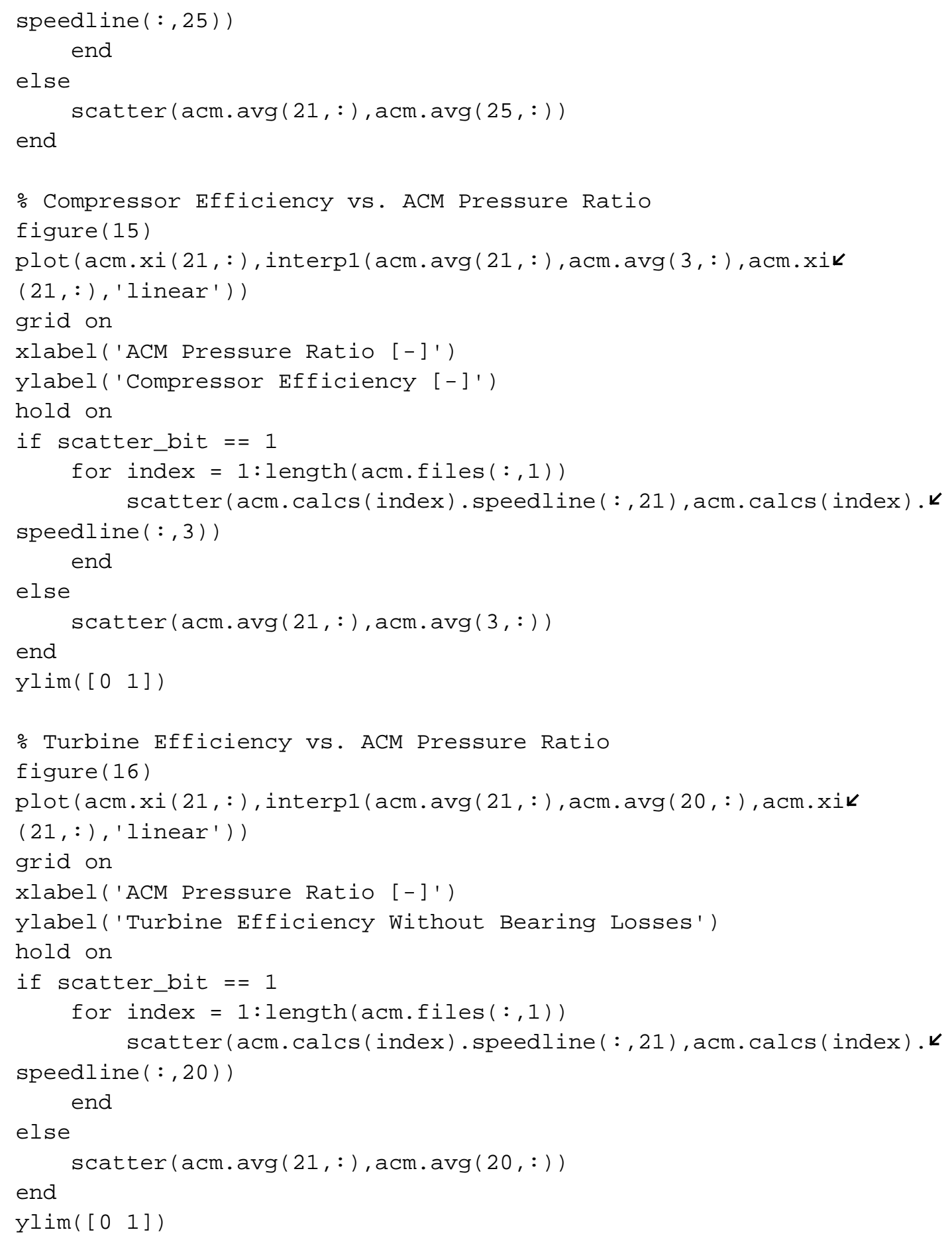


\%Program by Chris Forster

\%July 15, 2008

\%Input all humidity ratios in grains/lbm

\%Input temperature in degrees $\mathrm{F}$

\%Returns Dry Air Rated Temperature in degrees $\mathrm{F}$

function [T_DB] = T_DAR_DB (T_DAR,g_sat,g_tot $)$

$\%$ Define g_ent (entrained water)

if (g_tot $>=$ g_sat)

g_ent = g_tot - g_sat;

else

g_ent $=0$;

end

\% Convert grains to $1 \mathrm{bm}$

g_sat $=$ g_sat $/ 7000$;

g_tot $=$ g_tot $/ 7000$;

g_ent = g_ent $/ 7000$;

\% Calculate T_DB from T_DAR assuming above 32F (DB)

\% Check assumption afterwards.

T_DB_TBD $=\left(\right.$ T_DAR ${ }^{*}\left(0.24+0.4332^{*}\right.$ g_tot $)+1093^{*}$ g_ent $) /(0.24+0 . k$

4332*g_sat+g_ent);

if (T_DB_TBD $<=32$ )

T_DB_TBD $=\left(T \_D A R^{*}\left(0.24+\odot .4332^{*}\right.\right.$ g_tot $)+1221^{*}$ g_ent $) /(\odot .24+\odot . k$ $4332^{*}$ g_sat $+0.5^{*}$ g_ent $)$;

end

T_DB = T_DB_TBD; $\quad$ \%Degree $\mathrm{F}$

end 
\%Program by Chris Forster

\%April 16, 2008

\%Input all humidity ratios in grains/lbm

\%Input temperature in degrees $\mathrm{F}$

\%Returns Dry Air Rated Temperature in degrees $\mathrm{F}$

function[T_DAR] = T_DAR(T_DB,g_sat,g_tot $)$

if g_sat > g_tot

g_ent $=0$;

T_DAR = T_DB;

return

end

if g_sat $<$ g_tot

g_ent = g_tot - g_sat;

end

if $T$ _DB $>32$

$\mathrm{T}$ _DAR $=\left(\left(0.24+0.4332{ }^{*} \mathrm{~g} \_\right.\right.$sat $/ 7000+\mathrm{g} \_$ent $\left./ 7000\right){ }^{*} \mathrm{~T} \_\mathrm{DB}-\boldsymbol{K}$

$1093^{*}$ g_ent $\left./ 7000\right) /\left(0.24+0.4332{ }^{*}\right.$ g_tot $\left./ 7000\right)$;

end

if $T \_D B<=32$

T_DAR $=\left(\left(0.24+0.4332{ }^{*}\right.\right.$ g_sat $/ 7000+0.5^{*}$ g_ent $\left./ 7000\right){ }^{*} T$ DB $-\boldsymbol{k}$

$1221 *$ g_ent $/ 7000) /(0.24+0.4332 *$ g_tot $/ 7000)$;

end

end 


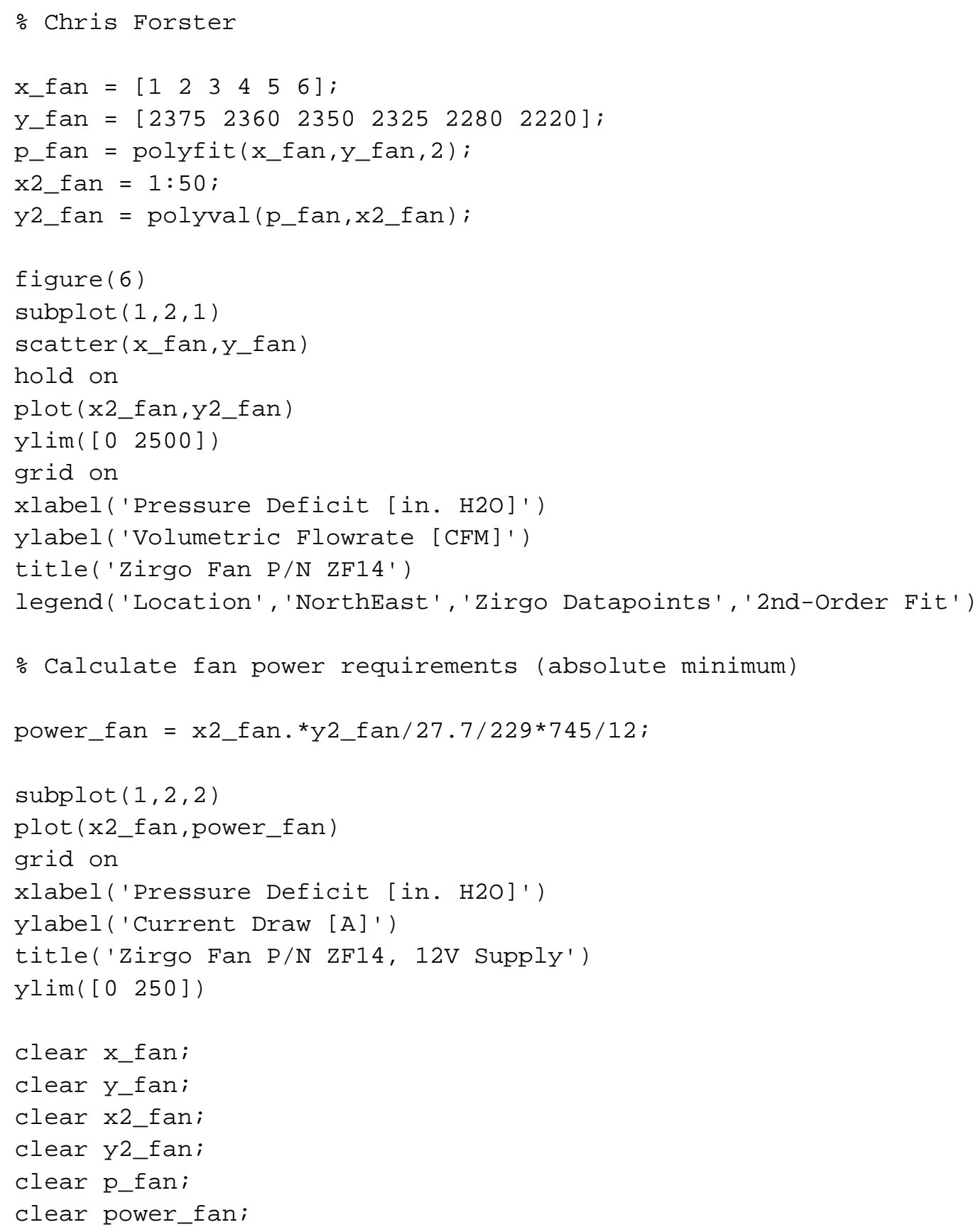




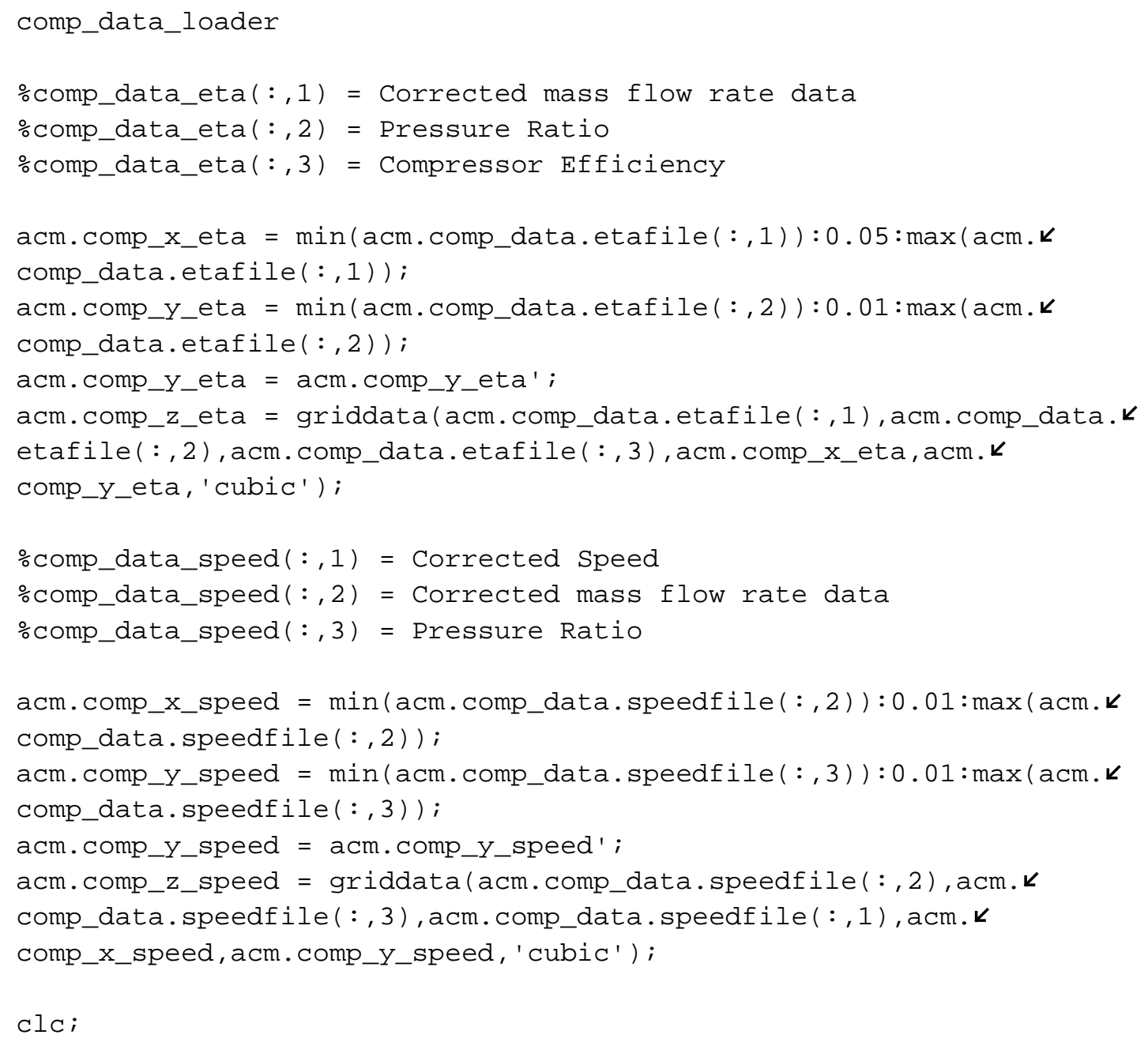


\%Chris Forster

\%The file format is a tab-delimited text file

\%This file will be run automatically...

acm.comp_data.speedfile = load('comp_data_speed.txt');

acm.comp_data.etafile = load('comp_data_eta.txt') ; 
\% Chris Forster

$\% 6 / 20 / 2009$

$\%$ This function is a viscosity correction factor for LFE measurements.

\% (Source: WwW.meriam.com)

function $[$ up] = viscosity_correction $(T)$

$v_{-} \operatorname{col1}=50: 159$;

v_col2 $=\left[\begin{array}{lllllllllll}1.03034 & 1.02877 & 1.02720 & 1.02564 & 1.02408 & 1.02253 & 1.02099 k\end{array}\right.$

$\begin{array}{llllllll}1.01945 & 1.01792 & 1.01639 & 1.01487 & 1.01336 & 1.01185 & 1.01035 & 1.00885 k\end{array}$

$1.007361 .005881 .004401 .00292 \ldots$

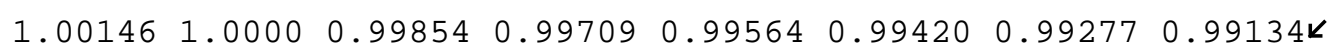

$\begin{array}{lllllllll}0.98992 & 0.98850 & 0.98709 & 0.98568 & 0.98428 & 0.98288 & 0.98149 & 0.98010 \mathrm{~K}\end{array}$

$0.97872 \quad 0.97734 \quad 0.97597 \quad 0.97461 \quad 0.97325 \quad \ldots$

$\begin{array}{llllllllll}0.97189 & 0.97054 & 0.96919 & 0.96785 & 0.96651 & 0.96518 & 0.96386 & 0.96253 \mathrm{~K}\end{array}$

$\begin{array}{llllllll}0.96122 & 0.95991 & 0.95860 & 0.95729 & 0.95600 & 0.95470 & 0.95341 & 0.95213 \mathrm{~K}\end{array}$

$0.950850 .94957 \quad 0.94830 \quad 0.94704 \ldots$

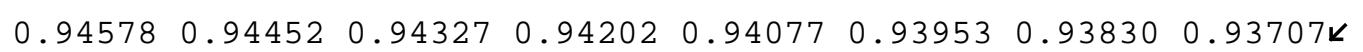

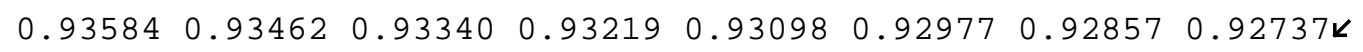

$0.92618 \quad 0.92499 \quad 0.92380 \quad 0.92262 \ldots$

$0.92144 \quad 0.92027 \quad 0.91910 \quad 0.91794 \quad 0.91678 \quad 0.91562 \quad 0.91446 \quad 0.91331 K$

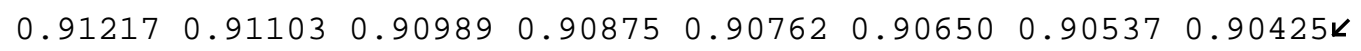

$0.903140 .90203 \quad 0.90092 \quad 0.89981 \ldots$

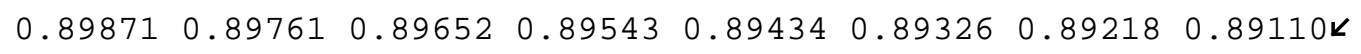

$\odot .89003$ ๑.88896];

v_table $=\left[\mathrm{v} \_\right.$col1' $1^{\prime} \_$col2' $]$;

up $=$ interp1 $\left(v_{-}\right.$table $(:, 1), v_{-}$table $\left.(:, 2), T\right)$;

end 


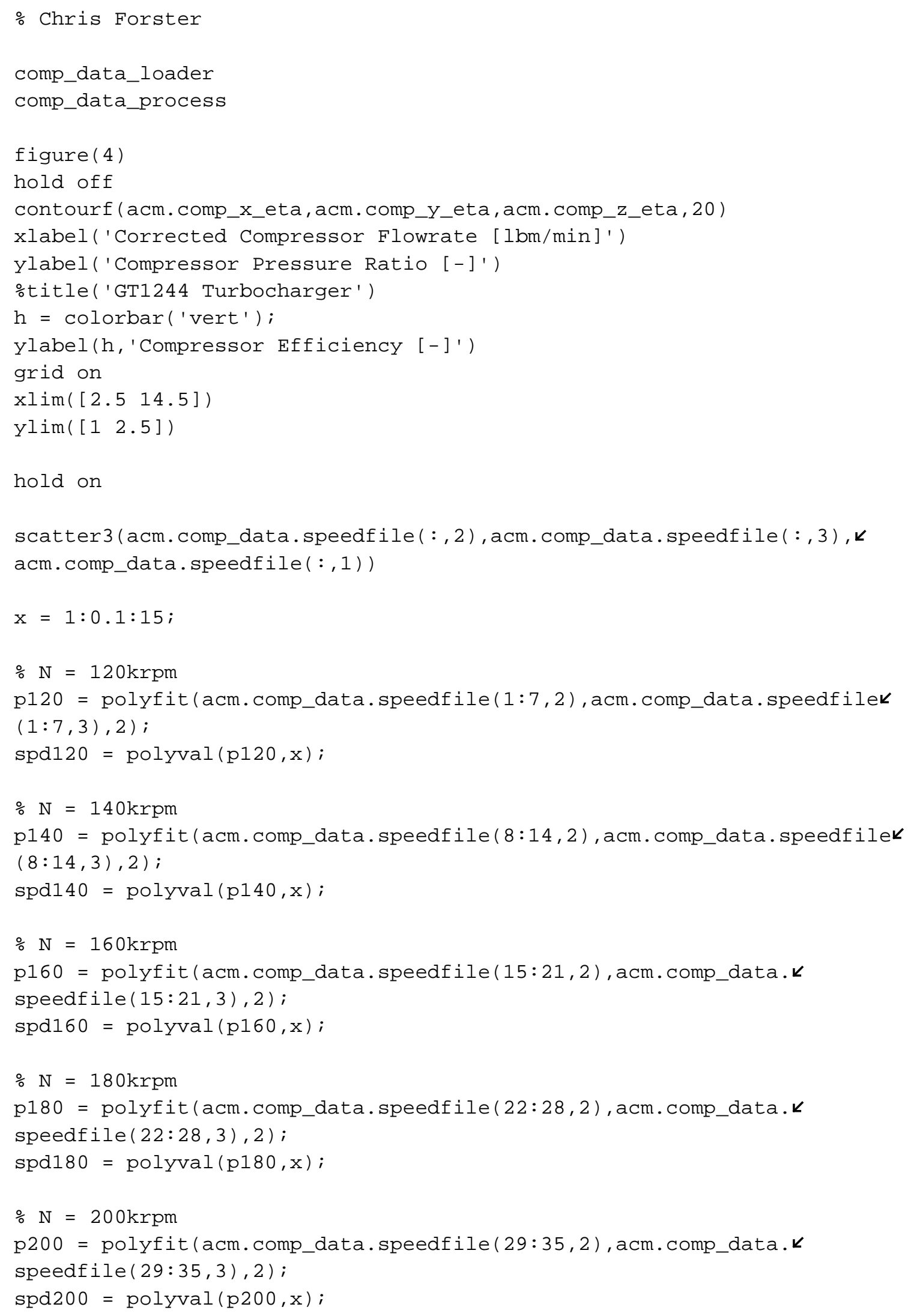


hold on

$\operatorname{plot}(x, \operatorname{spd} 120, x, \operatorname{spd} 140, x, \operatorname{spd} 160, x, \operatorname{spd} 180, x, \operatorname{spd} 200)$

$\mathrm{g}=$ legend('Location', 'NorthEast', 'Compressor Efficiency', 'Actualk

Speed Points', '120krpm', '140krpm', '160krpm', '180krpm', '200krpm');

hold off 
Appendix N - Perkins Diesel Post-processing Code in Matlab 


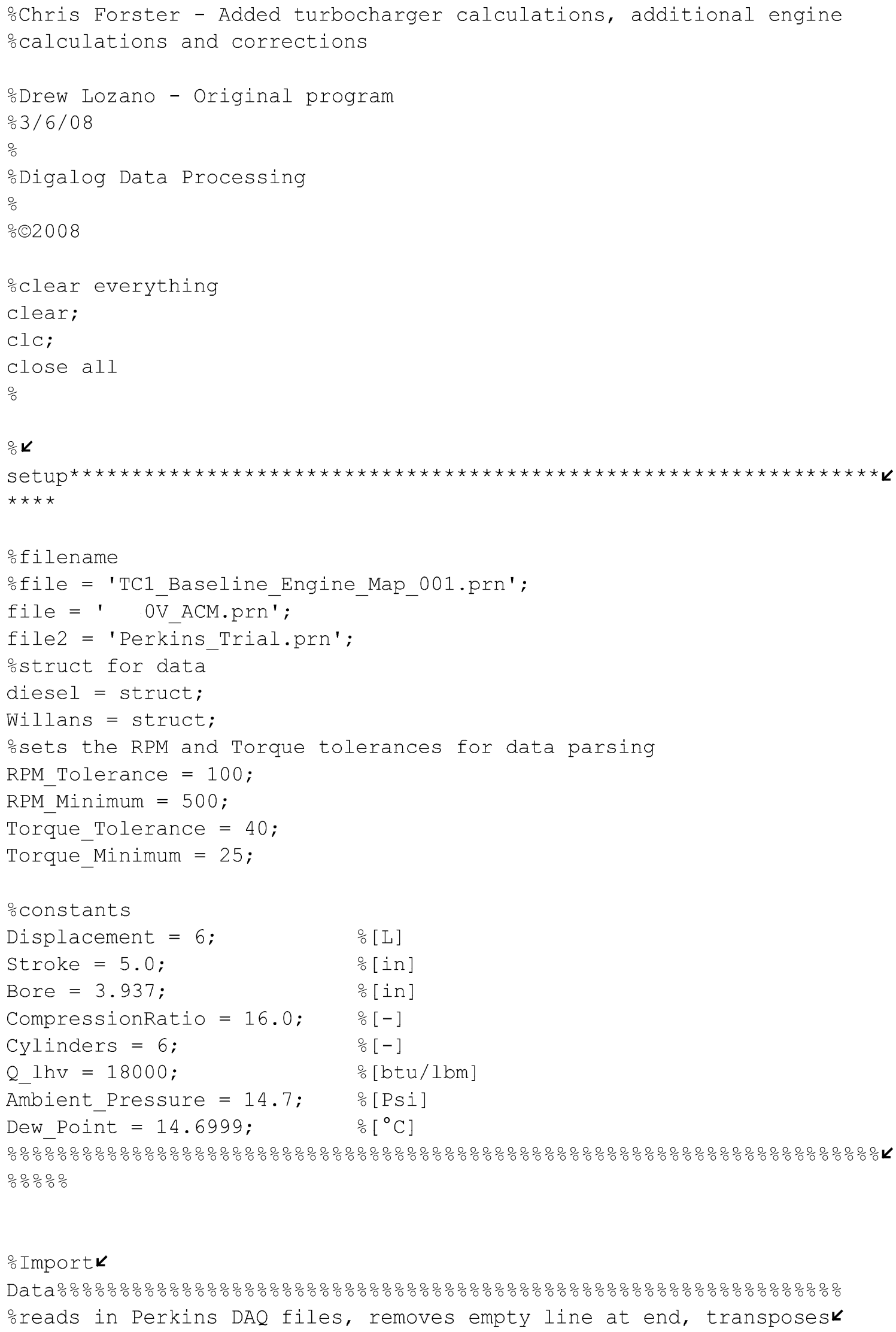




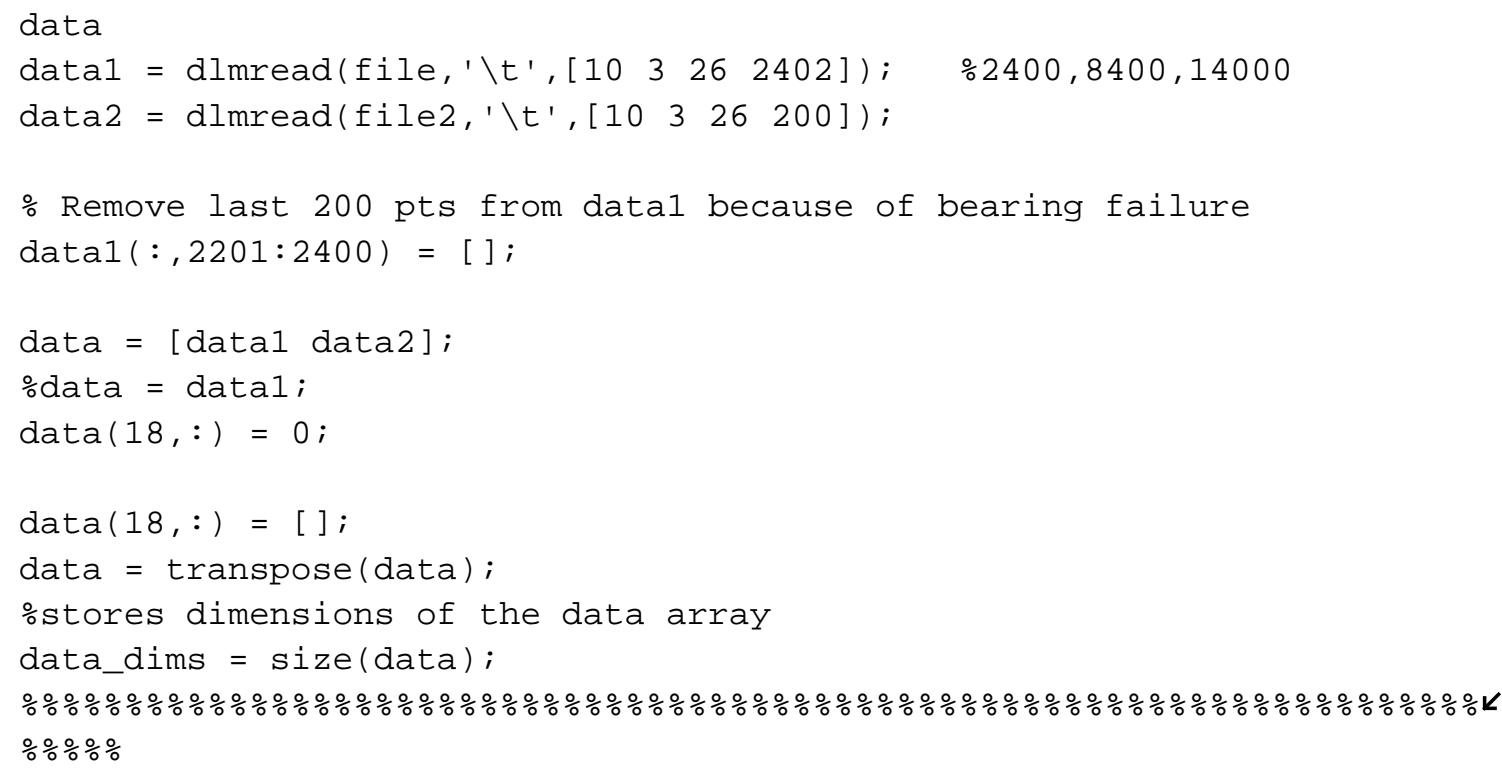




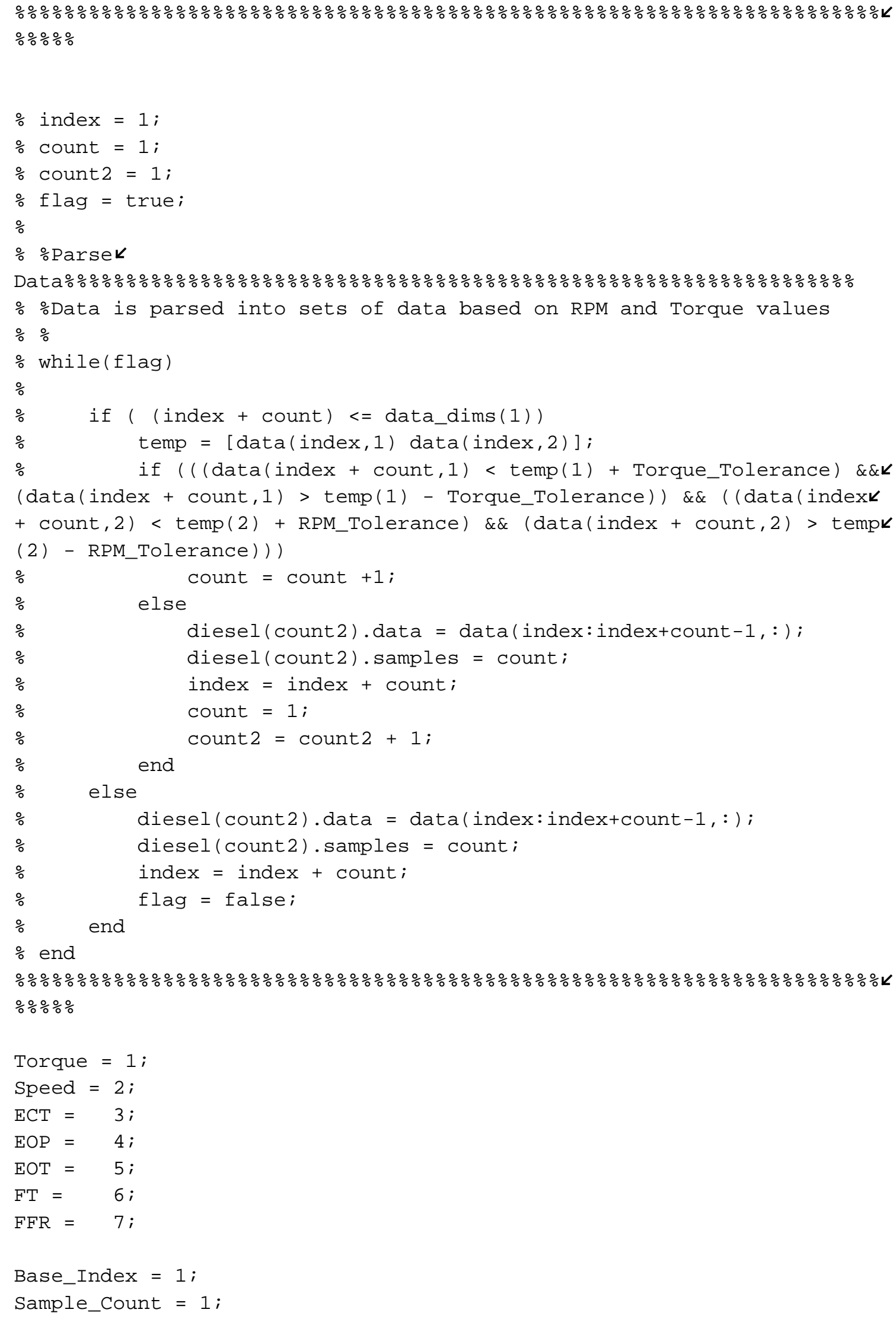




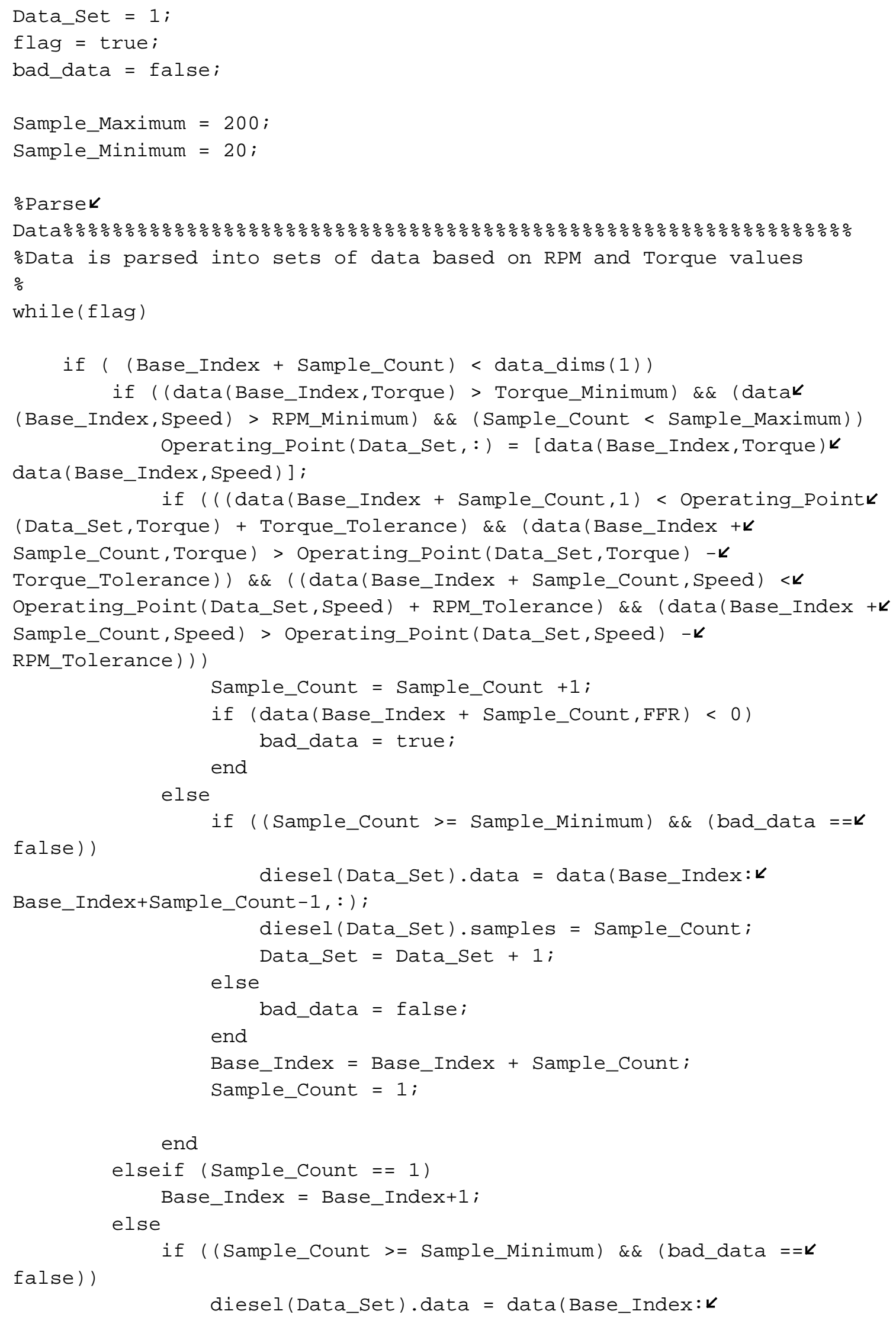




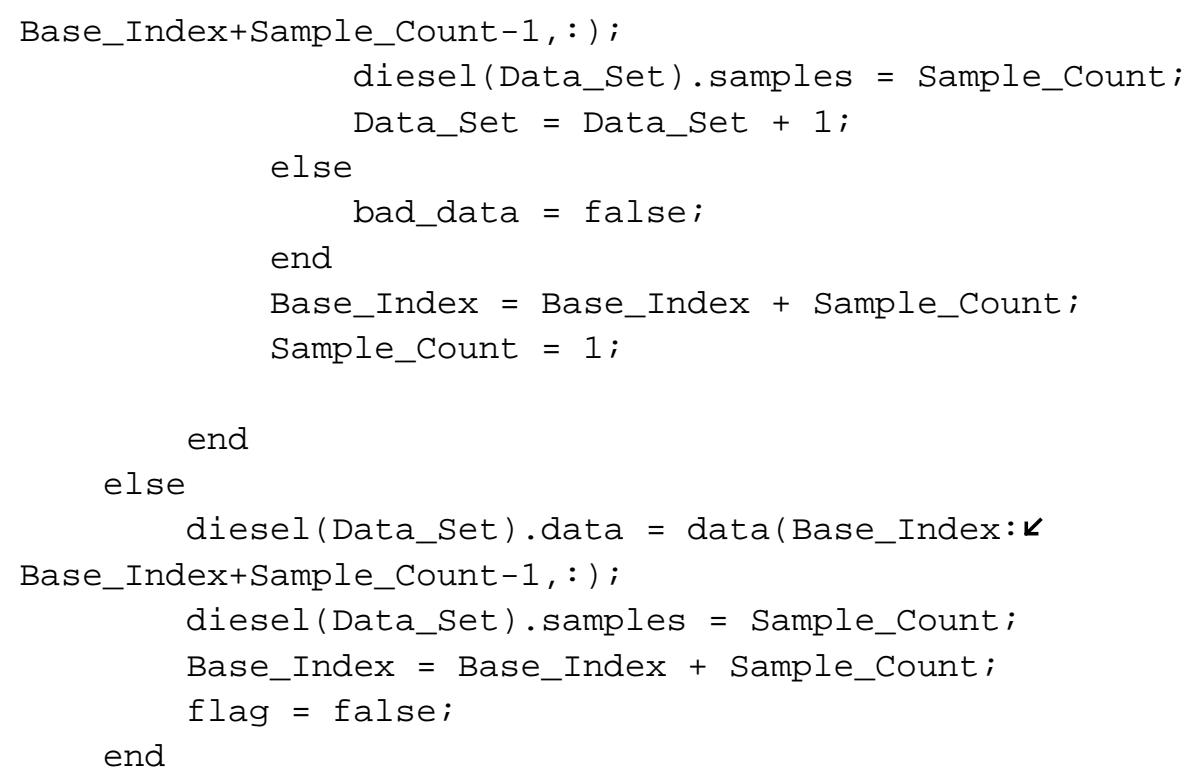

end

diesel_dims = size $($ diesel $) ;$

\%\% Append ACM Test Data to the Perkins Dataset //Added by Chrisk Forster

file_acm = 'acm_avg.txt';

data_acm = dlmread $\left(f i l e \_a c m, ' \backslash t '\right)$;

$\%$ Remove bad dataset for bearing failure

data_acm $(:, 12)=[]$;

for count $=1$ :diesel_dims $(2)$

diesel (count) $\cdot$ data $(:, 18)=$ data_acm $(1$, count $)$;

diesel (count).data $(:, 19)=$ data_acm $(2$, count $)$;

diesel(count).data $(:, 20)=$ data_acm $(3$, count $)$;

diesel (count).data $(:, 21)=$ data_acm $(4$, count $)$;

diesel (count).data $(:, 22)=$ data_acm $(5$, count $)$;

diesel (count) $\cdot$ data $(:, 23)=$ data_acm $(6$, count $)$;

diesel (count).data $(:, 24)=$ data_acm $(7$, count $)$;

diesel (count) $\cdot$ data $(:, 25)=$ data_acm $(8$, count $)$;

diesel (count).data $(:, 26)=$ data_acm $(9$, count $)$;

diesel (count).data $(:, 27)=$ data_acm $(10$, count $)$;

diesel(count).data $(:, 28)=$ data_acm $(11$, count);

diesel (count).data $(:, 29)=$ data_acm $(12$, count $)$;

diesel(count).data $(:, 30)=$ data_acm $(13$, count $)$;

diesel (count).data $(:, 31)=$ data_acm $(14$, count $)$;

diesel (count).data $(:, 32)=$ data_acm $(15$, count $)$;

diesel(count).data $(:, 33)=$ data_acm $(16$, count $)$; 


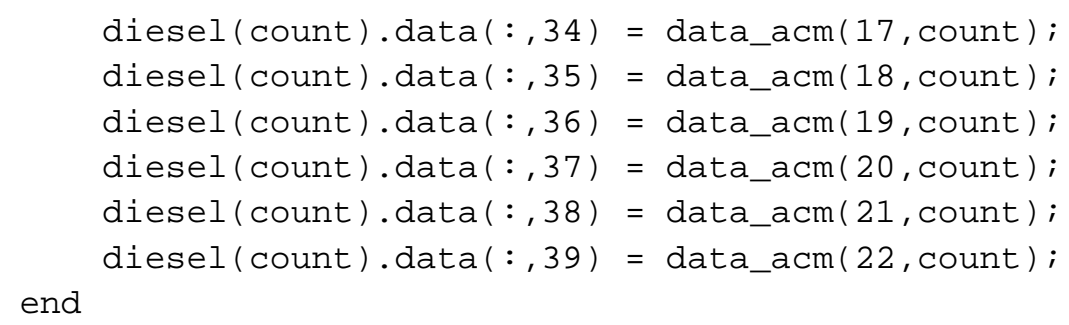


for count $=1$ :diesel_dims $(2)$

for count2 = 1:diesel( count). samples

diesel(count). calcs (count 2,5$)=2 * \operatorname{diesel}($ count) $\cdot$ data (count $2, k$

17) / ( $0.035314666919 *$ Displacement * diesel(count).data(count2, 2)); end

end

\%Inlet Volumetric Efficiency Calculations

for count = 1:diesel_dims $(2)$

for count $2=1$ : diesel (count). samples

$\%$ diesel (count) $\cdot \operatorname{calcs}($ count 2,6$)=2304 *$ diesel $($ count $) \cdot$ datak $($ count 2,17$) /($ Stroke * Bore^2 * pi * diesel(count).data (count2, 2)) *k (Ambient_Pressure / (Ambient_Pressure + diesel(count).data(count2, $k$ $10))) *($ diesel (count).data (count2,11) + 459.67) / (diesel(count).datak (count 2,8$)+459.67)$; diesel(count). calcs $($ count 2,6$)=$ Ambient_Pressure/(diesel $k$

(count). data (count2,10)+Ambient_Pressure)* (diesel (count). data (count2, $k$ 11) +460$) /($ diesel (count) . data (count2, 8) +460$) *$ diesel (count) . data (count $2, k$ $17) /\left(0.25^{*} \mathrm{pi}^{*}\right.$ Bore^${ }^{\star}{ }^{*}$ Stroke ${ }^{*}$ Cylinders $/ 1728^{*}$ diesel (count) . data (count2, 2$) k$ /2);

end

end

\%Air Mass Flow Rate Calculations

for count $=1$ :diesel_dims $(2)$

for count2 = $1:$ diesel $($ count $)$. samples

diesel(count). calcs $($ count 2,7$)=2.699504268 *$ diesel (count).$k$ data(count2,17) * Ambient_Pressure / ( diesel(count).data(count2,8) +k 459.67);

end

end

\%Mean Piston Speed Calculations

for count = 1:diesel_dims $(2)$

for count $2=1$ : diesel (count). samples

diesel(count) $\cdot$ calcs $($ count 2,8$)=2 / 60 *($ Stroke/12) * diesel $\kappa$

( count).data(count2,2);

end

end

\%BMEP Calculations

for count = 1:diesel_dims $(2)$

for count $2=1:$ diesel $($ count $)$. samples

diesel (count) $\cdot \operatorname{calcs}($ count 2,9$)=\operatorname{diesel}($ count $) \cdot \operatorname{calcs}(\operatorname{count} 2,1) k$

* 2 * $396000 /(6$ * Stroke * pi * (Bore^2 /4) *diesel(count). datak

( count 2, 2));

end

end 


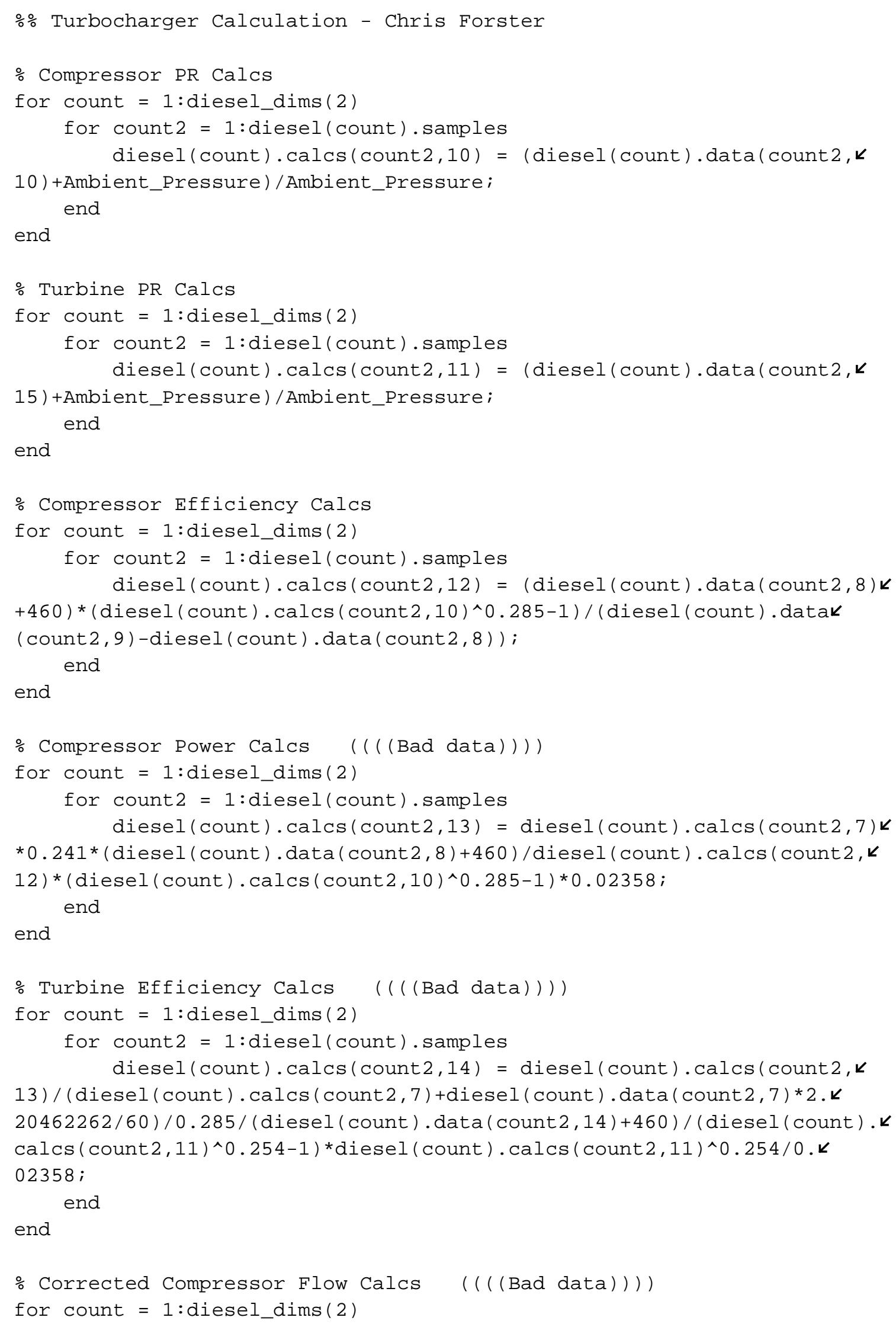




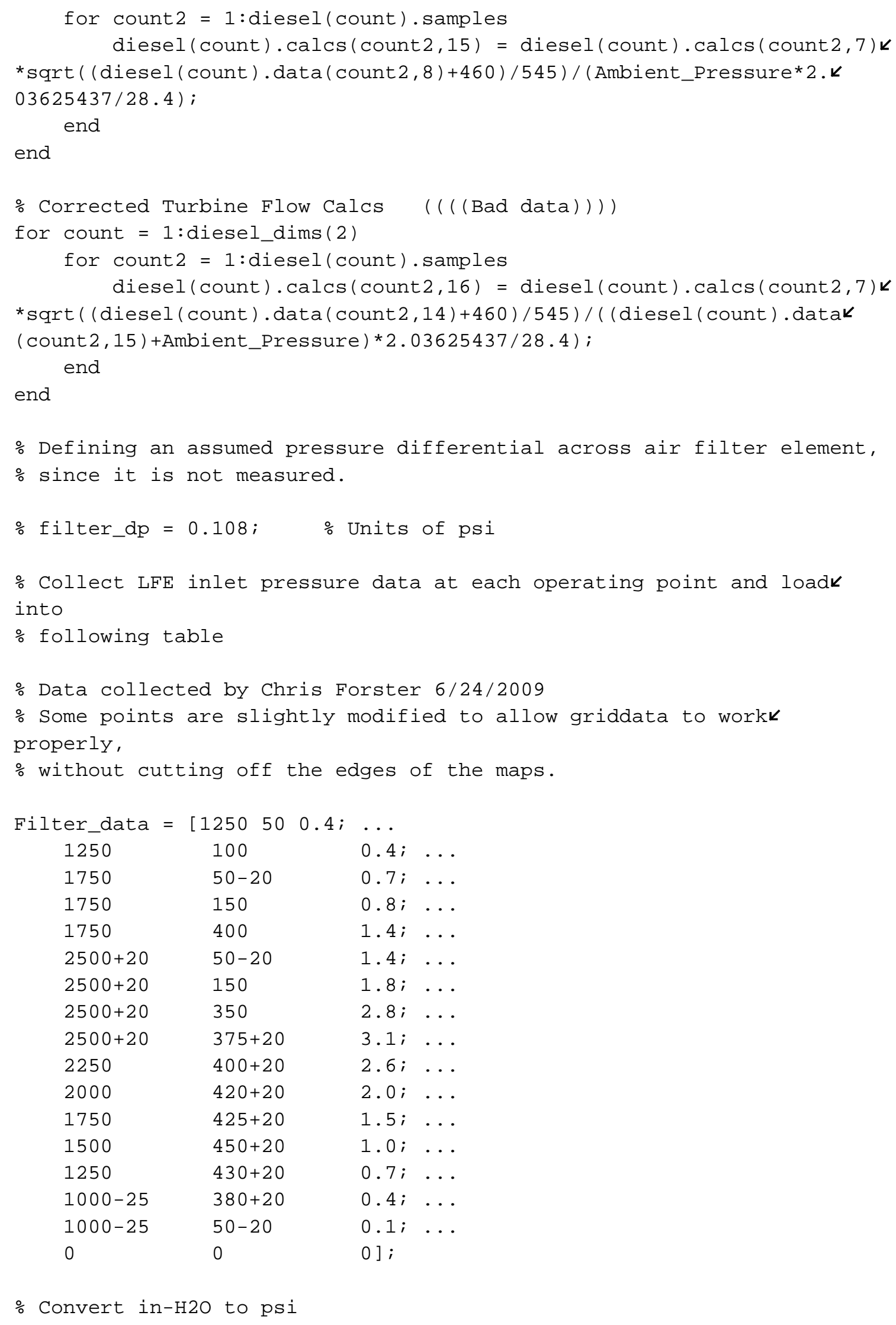




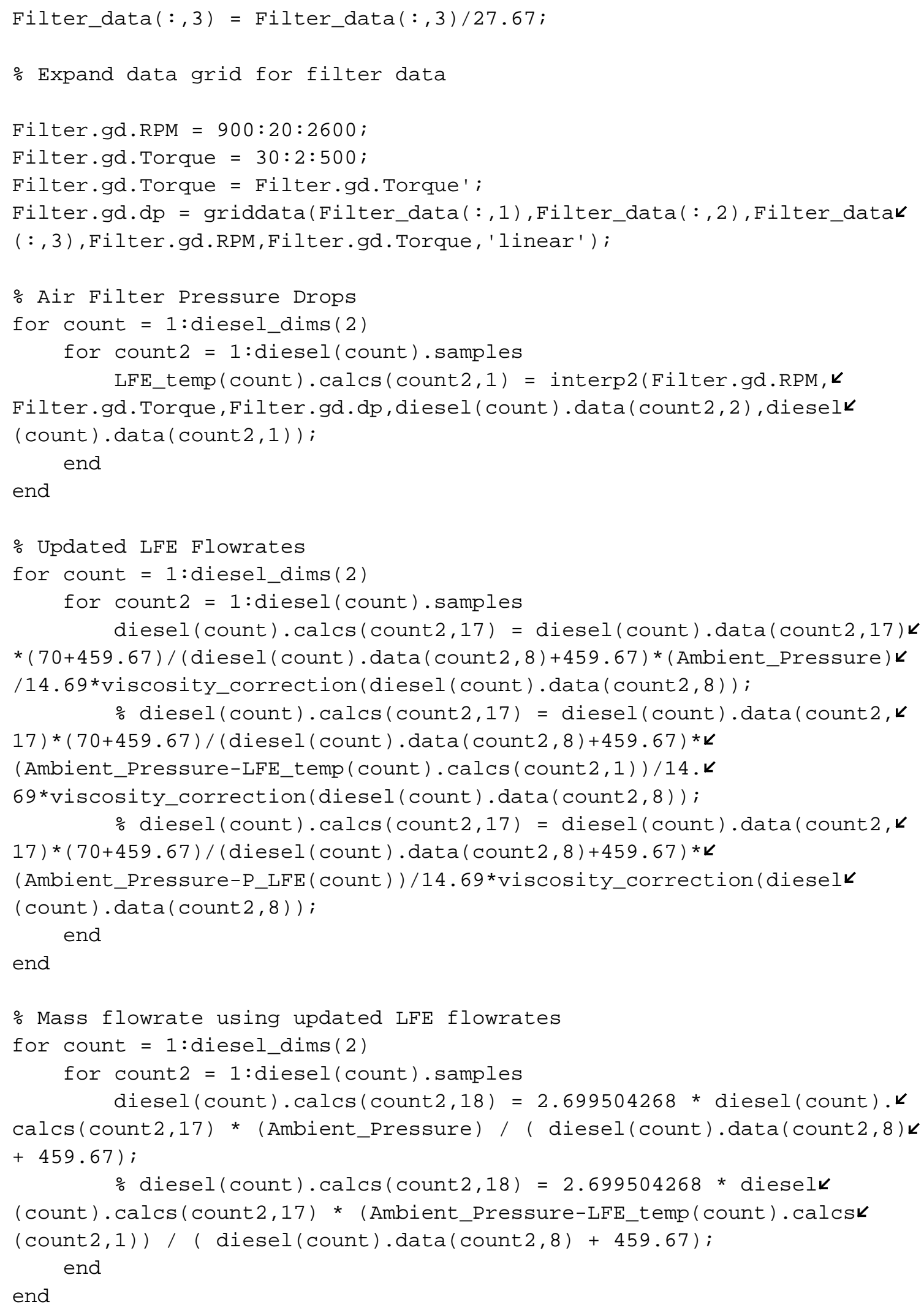


\% Intake Air Density using updated LFE flowrates (Compressor Inlet)

for count $=1$ : diesel_dims $(2)$

for count $2=1:$ diesel (count). samples

diesel (count). calcs $($ count 2,19$)=2.699504268{ }^{*} K$

(Ambient_Pressure) / ( diesel(count).data (count2,8) + 459.67);

$\%$ diesel (count) $\cdot \operatorname{calcs}($ count 2,19$)=2.699504268{ }^{*} \boldsymbol{k}$

(Ambient_Pressure-LFE_temp(count).calcs (count 2,1$)) /($ diesel (count).k

data (count2,8) + 459.67);

end

end

\% Manifold Volumetric Efficiency using updated LFE flowrates

for count $=1$ : diesel_dims(2)

for count $2=1:$ diesel (count). samples

diesel(count). calcs (count 2,20$)=$ diesel (count). calcs (count $2, k$

18) /( ( $0.25^{*} \mathrm{pi}^{*}$ Bore ${ }^{\wedge}{ }^{*}$ Stroke ${ }^{*}$ Cylinders $/ 1728^{*}$ diesel (count). data (count $2, k$

$2) / 2$ )*2.699504268* (diesel(count). data(count2,10)+Ambient_Pressure) / ( $k$

diesel(count).data(count2,8) + 459.67));

end

end

\% Exhaust Manifold Exhaust Density Calculations

for count $=1:$ diesel_dims $(2)$

for count $2=1$ :diesel (count). samples

diesel (count). calcs $($ count 2,21$)=2.699504268 *(1.122 / 0.8456){ }^{*} \boldsymbol{K}$

(Ambient_Pressure+diesel(count).data(count2,15)) / ( diesel(count).k

data(count2,14) + 459.67);

end

end

\% Exhaust Manifold Volumetric Flowrate

for count $=1:$ diesel_dims $(2)$

for count $2=1$ :diesel (count). samples

diesel (count) $\cdot \operatorname{calcs}($ count 2,22$)=($ diesel $($ count $) \cdot \operatorname{calcs}($ count $2, k$

$18)+$ diesel (count) $\cdot$ data (count 2,7$\left.){ }^{\star} \odot \cdot 0367437104\right) /$ diesel (count) . calcsk

( count 2, 21);

end

end

\% Exhaust Manifold - Pumping Losses (hp)

for count $=1$ :diesel_dims $(2)$

for count $2=1$ : diesel (count). samples

diesel(count) $\cdot$ calcs (count2,23) $=0.00436363636$ * diesel $\boldsymbol{k}$

(count). calcs (count2, 22)*diesel (count) . data (count2, 15);

end

end

\% Compressor Efficiency Calcs (using updated LFE values...doesn'tk affect this one) 


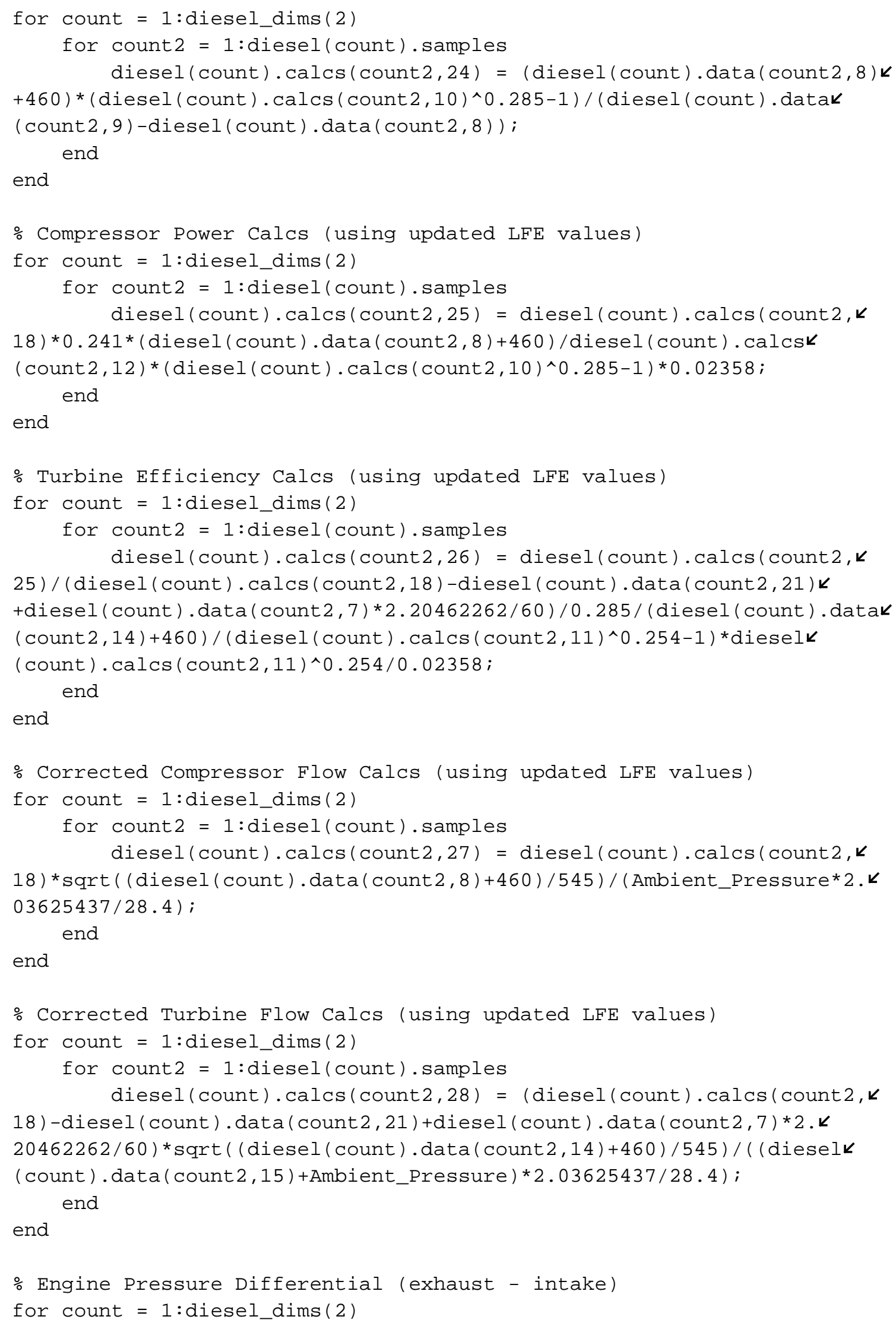




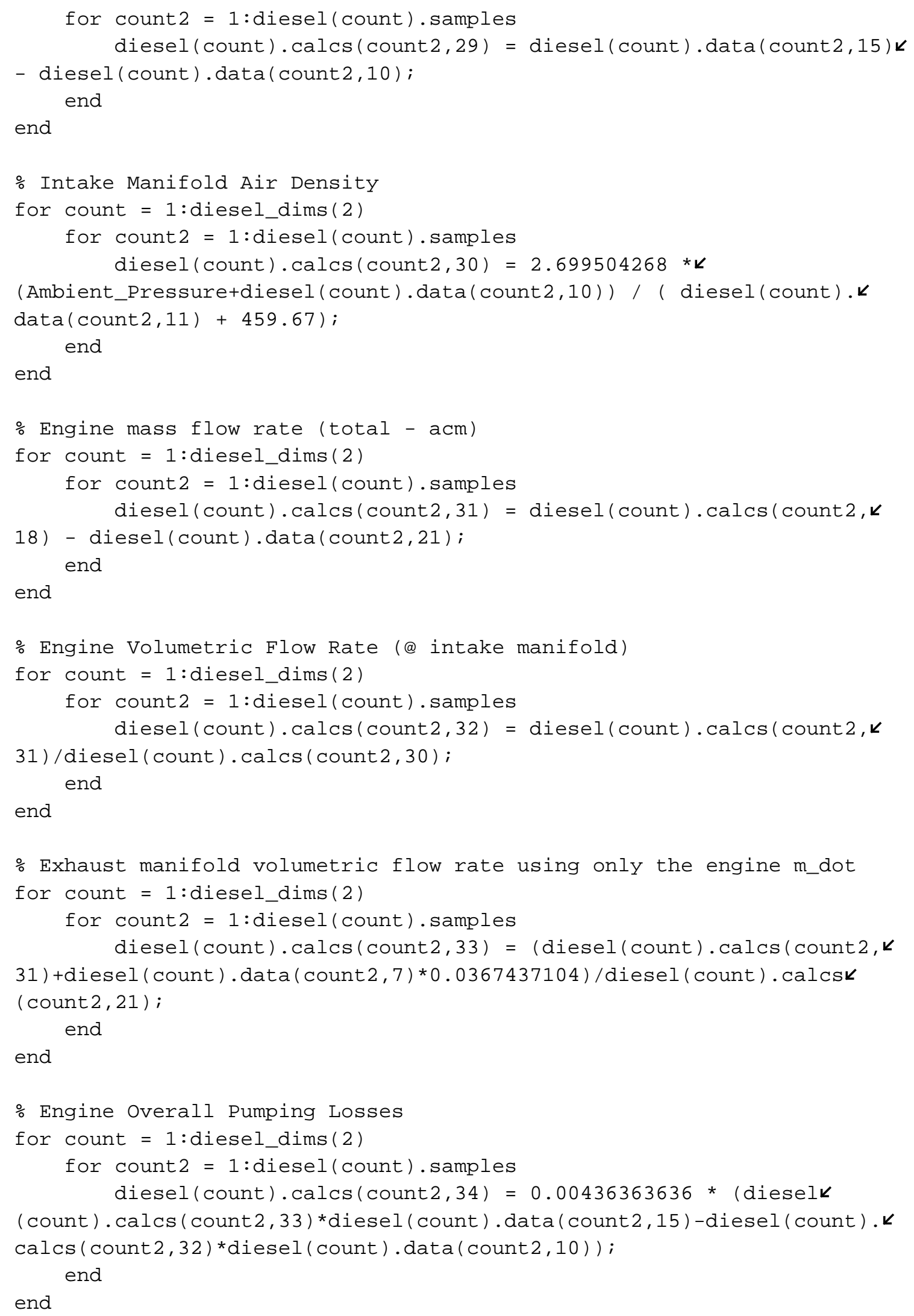


\% End Turbocharger Calculations

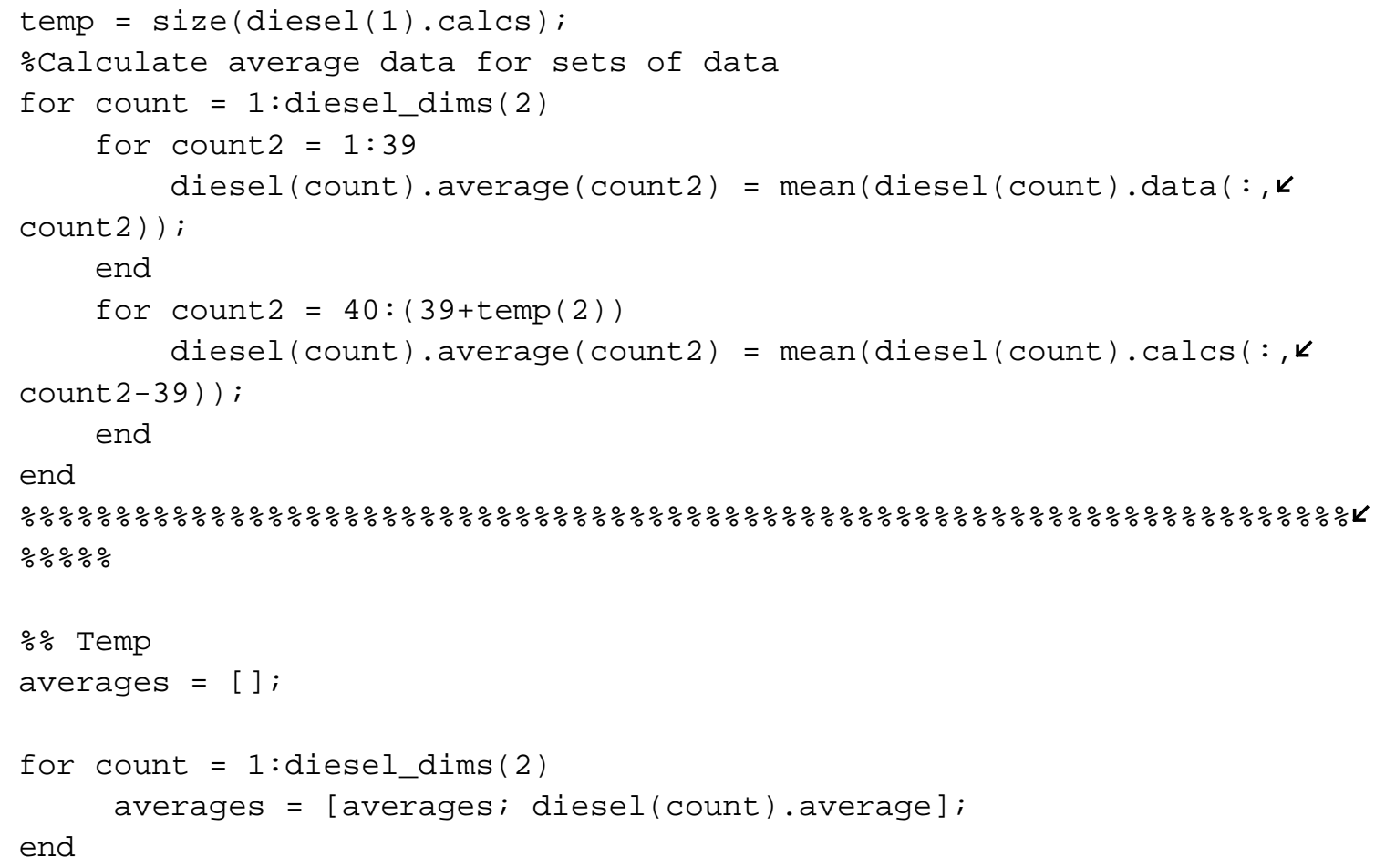


Added by Chris Forster

TC.W_T_C (count $)=$ diesel $($ count $)$. average $(33+22) ; \quad \% k$

Added by Chris Forster

TC.T1T $($ count $)=$ diesel $($ count $) \cdot \operatorname{average}(14)$;

by Chris Forster

IC_eff (count) $=$ diesel (count) . average $(21+22)$;

\%Addedk

Added by Chris Forster

IC_Tout $($ count $)=$ diesel (count). average (11);

by Chris Forster

TC.W_phy $($ count $)=$ diesel $($ count $) \cdot \operatorname{average}(24+22)$;

Added by Chris Forster

TC.VE_man (count) $=$ diesel (count) $\cdot \operatorname{average}(23+22)$;

Added by Chris Forster

TC.W_fuel (count) $=$ diesel $($ count $) \cdot \operatorname{average}(7) * 0.0367437104 ;$

by Chris Forster

TC. Boost $($ count $)=$ diesel $($ count $) \cdot \operatorname{average}(10)$;

by Chris Forster

TC.T_amb $($ count $)=$ diesel $($ count $) \cdot \operatorname{average}(8)$;

by Chris Forster

TC.W_phy_updated $($ count $)=$ diesel $($ count $) \cdot \operatorname{average}(35+22)$;

Added by Chris Forster

TC.VE_man_updated (count) $=$ diesel (count). average $(37+22)$;

Added by Chris Forster

TC. EGD (count) = diesel (count). average $(38+22)$;

Added by Chris Forster

TC.Pumping (count) $=$ diesel (count) $\cdot \operatorname{average}(39+22)$;

Added by Chris Forster

TC.EGP( count $)=$ diesel (count). average $(15)$;

by Chris Forster

TC.ETA_C_updated (count) $=$ diesel (count). average $(41+22)$;

Added by Chris Forster

TC.P_C_updated $($ count $)=$ diesel $($ count $) \cdot \operatorname{average}(42+22)$;

Added by Chris Forster

TC.ETA_T_updated $($ count $)=$ diesel $($ count $) \cdot \operatorname{average}(43+22)$;

Added by Chris Forster

TC.W_C_C_updated (count) $=$ diesel (count) . average $(44+22)$;

Added by Chris Forster

TC.W_T_C_updated $($ count $)=$ diesel (count) . average $(45+22)$;

Added by Chris Forster

TC.Vflow_updated (count) $=$ diesel (count) . average $(34+22)$;

Added by Chris Forster

TC. $\operatorname{EDP}($ count $)=$ diesel $($ count $) \cdot \operatorname{average}(46+22)$;

Added by Chris Forster

TC.Pumping_dp (count) $=$ diesel $($ count $) \cdot \operatorname{average}(51+22)$;

Added by Chris Forster

ACM.DARcap (count) $=$ diesel (count) $\cdot \operatorname{average}(32)$;

Added by Chris Forster

end 
\%Determines the RPM set points used

$\%$ Creates a vector of RPM set points

clear temp;

temp $=\operatorname{sort}(\mathrm{RPM})$;

count $=1$;

for count $2=1$ : diesel_dims $(2)$

if $(($ count $==1)$ \&\& (temp $($ count2) > RPM_Minimum $))$

Base_RPM(count) $=$ temp $($ count 2$)$;

count $=$ count +1 ;

count $2=$ count $2+1 ;$

else if $(($ count $>1) \& \&$ (temp (count2) > Base_RPM (count -1$)+k$

RPM_Tolerance))

Base_RPM (count) $=$ temp $($ count 2$) ;$

count $=$ count +1 ;

end

end

end

\%Determines the Torque set points used

\%creates a vector of Torque set points

clear temp;

temp $=\operatorname{sort}($ Torque $)$;

count $=1$;

for count2 $=1$ : diesel_dims $(2)$

if ( (count $==1)$ \&\& (temp (count2) > Torque_Minimum) )

Base_Torque (count) $=$ temp $($ count 2$)$;

count $=$ count +1 ;

count $2=$ count $2+1 ;$

else if $(($ count $>1) \& \&($ temp $($ count 2$)>$ Base_Torque (count -1$)+K$

Torque_Tolerance/2))

Base_Torque $($ count $)=$ temp $($ count 2$)$;

count $=$ count +1 ;

end

end

end

\%Determines the dimensions of the useful averaged data matrix

RPM_Points = size(Base_RPM);

Torque_Points = size (Base_Torque);

\%Determines the position of the BSFC data point in the RPM/Torquek matrix

\%based on its RPM and Torque set points

for count $=1$ :diesel_dims $(2)$

for count2 = 1:RPM_Points(2)

for count3 = 1:Torque_Points $(2)$

if $((($ RPM $($ count $)>($ Base_RPM (count2) - RPM_Tolerance/2))k

$\& \&($ RPM $($ count $)<($ Base_RPM $($ count 2$)+$ RPM_Tolerance/2) $)) \& \&(($ Torquek 


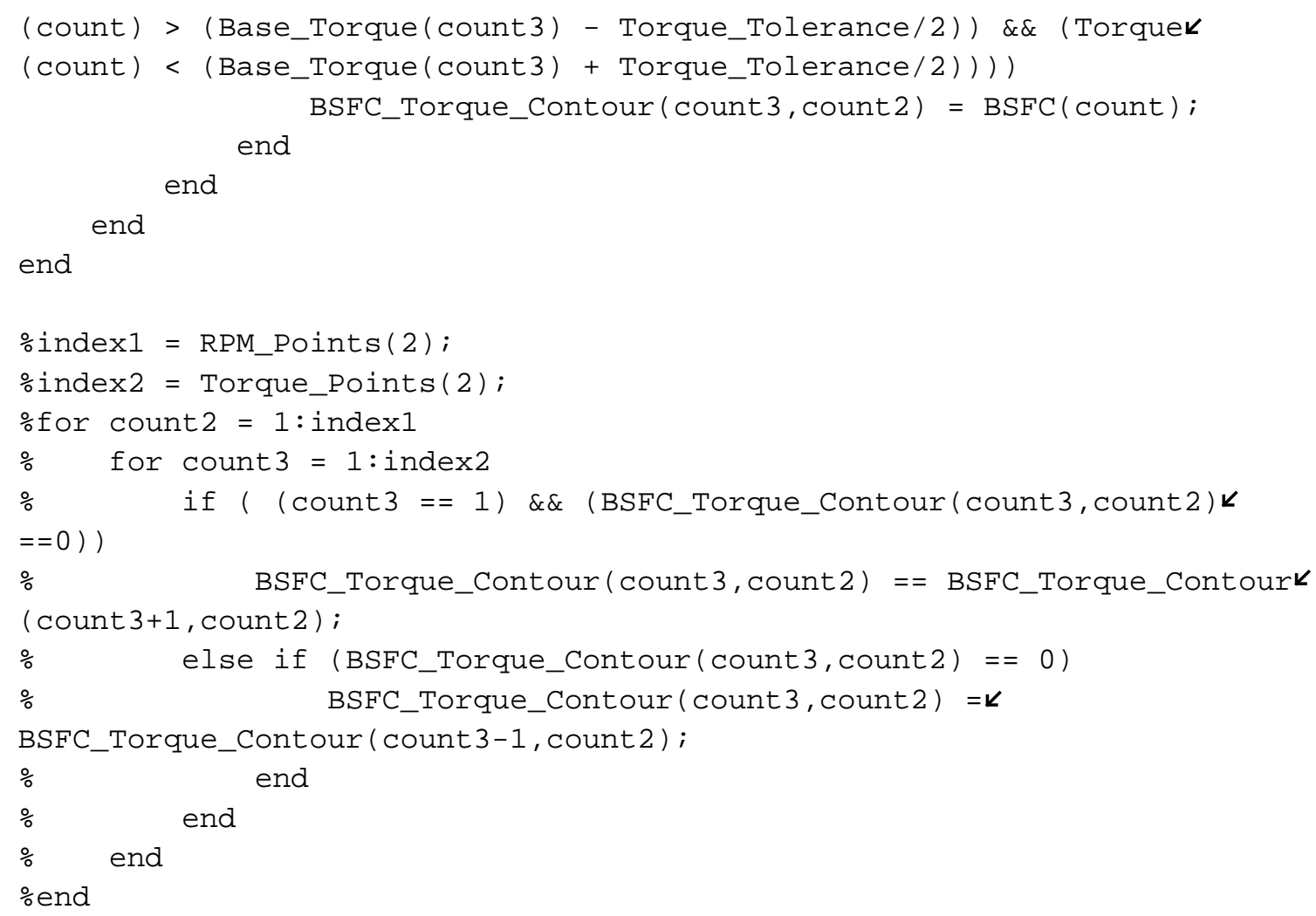




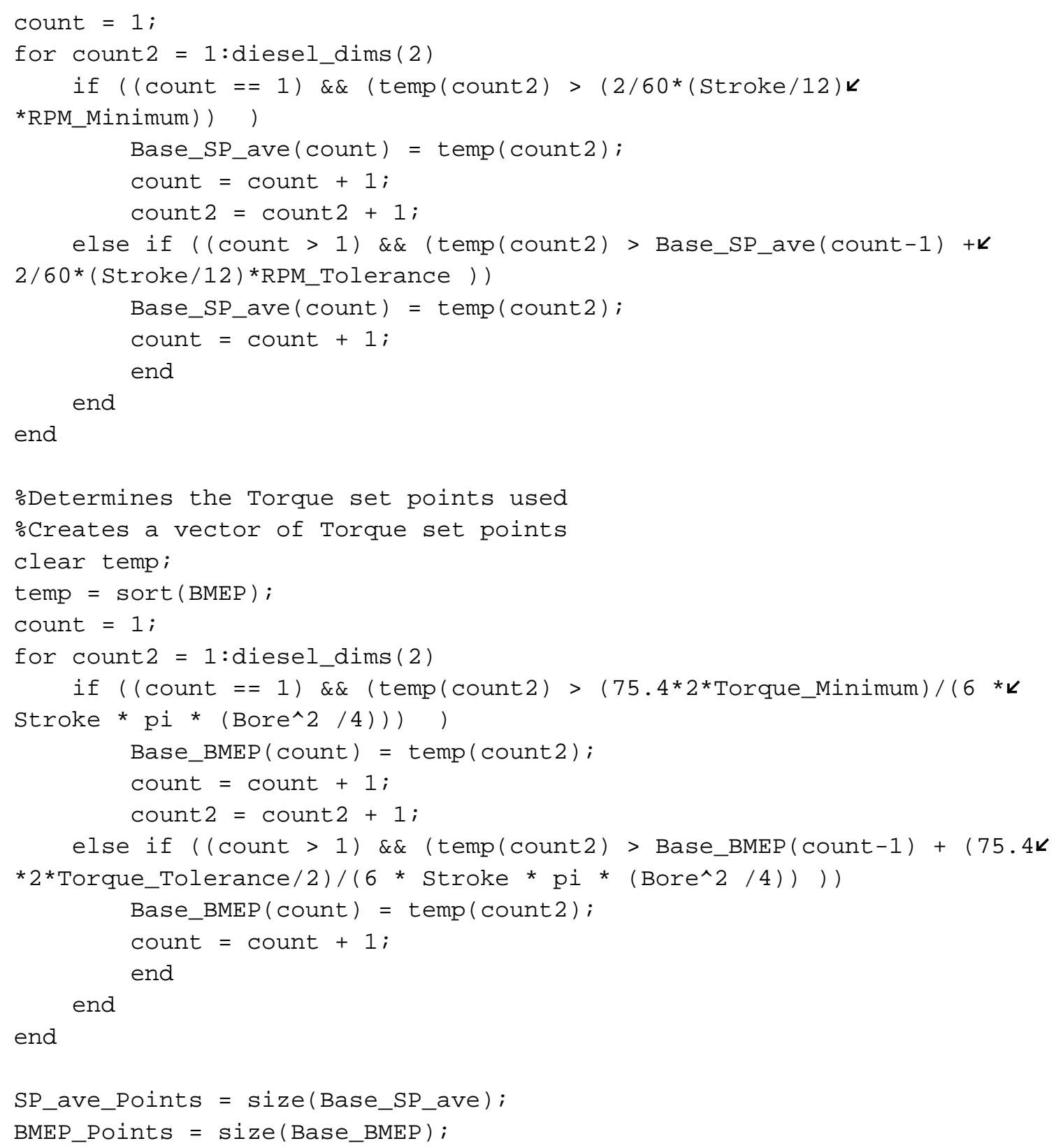

\%Determines the position of the BSFC data point in the SP_ave/BMEPK matrix

\%based on its RPM and Torque set points

for count $=1$ :diesel_dims(2)

for count2 = 1:SP_ave_Points(2)

for count3 = 1:BMEP_Points $(2)$ if $\left(\left(\right.\right.$ SP_ave (count) $>$ (Base_SP_ave (count2) - $\left(2 / 60^{*} K\right.$ $($ Stroke/12) * RPM_Tolerance/2)) ) \&\& (SP_ave (count) < (Base_SP_avek $($ count 2$)+\left(2 / 60^{*}\left(\right.\right.$ Stroke/12) ${ }^{*}$ RPM_Tolerance/2) $\left.\left.)\right)\right) \& \&((\operatorname{BMEP}($ count $)>k$ (Base_BMEP $($ count 3$)-\left(75.4 * 2 * T o r q u e \_T o l e r a n c e / 2\right) /(6$ * Stroke * pi *

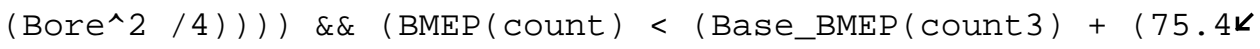




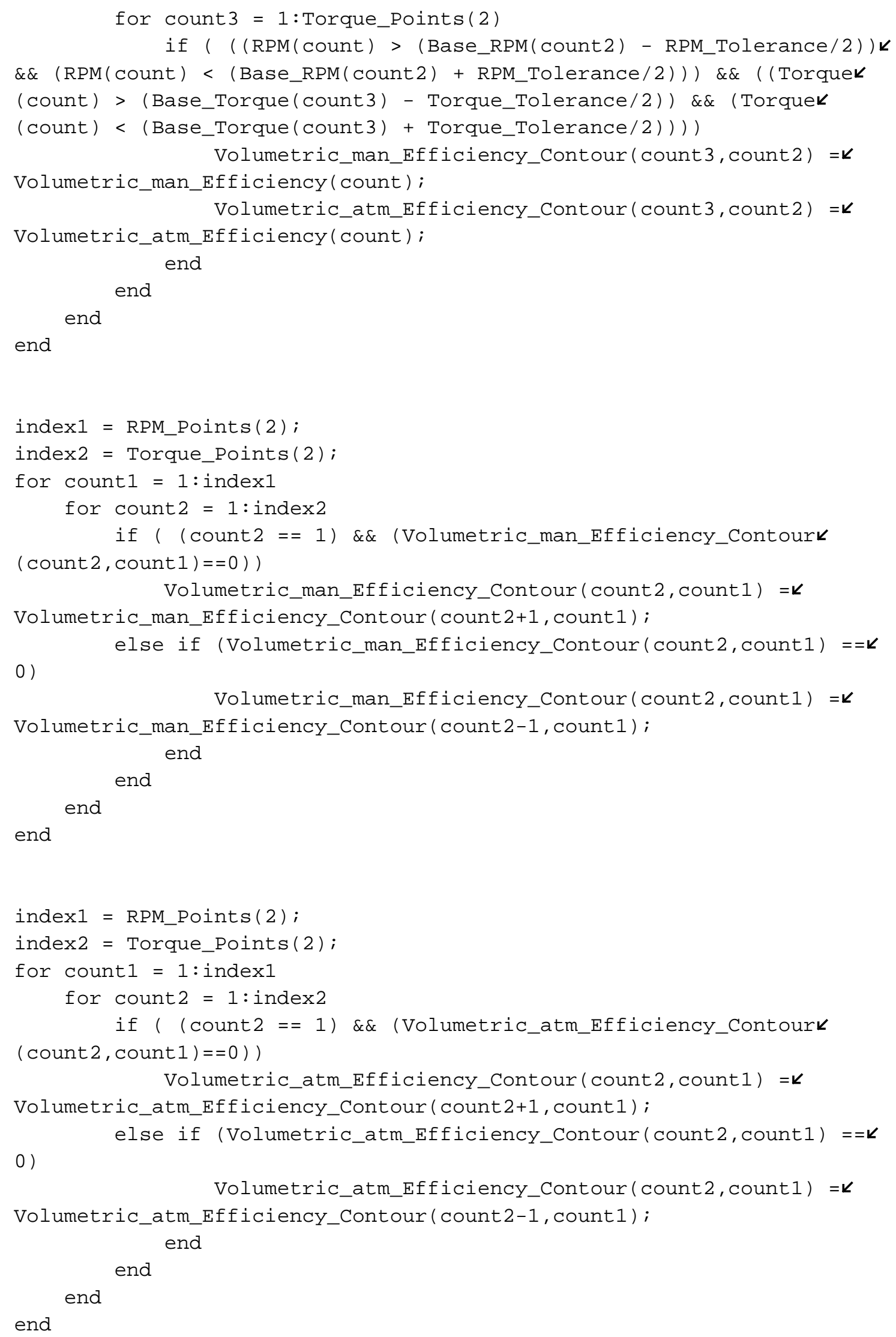




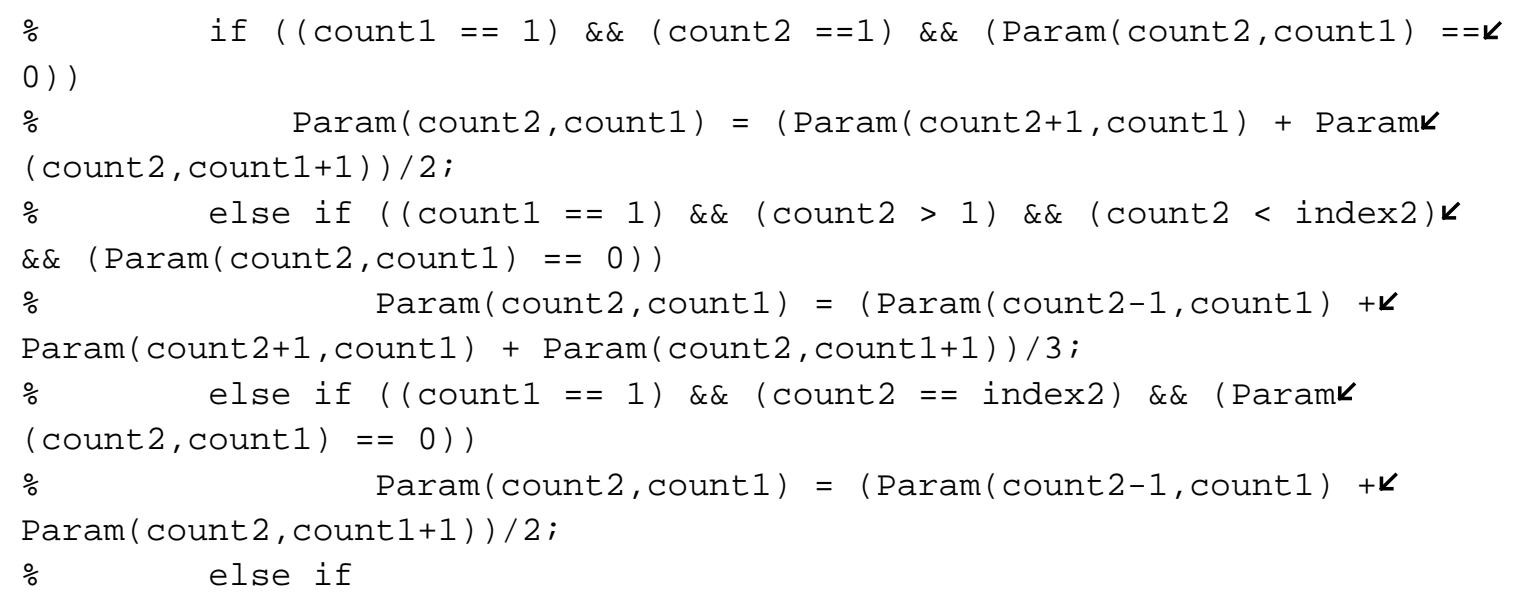


$\%$ title('Volumetric Efficiency (atmospheric)');

$\%$ xlabel('Engine Speed ( $r p m) ')$;

$\%$ ylabel('Volumetric Efficiency');

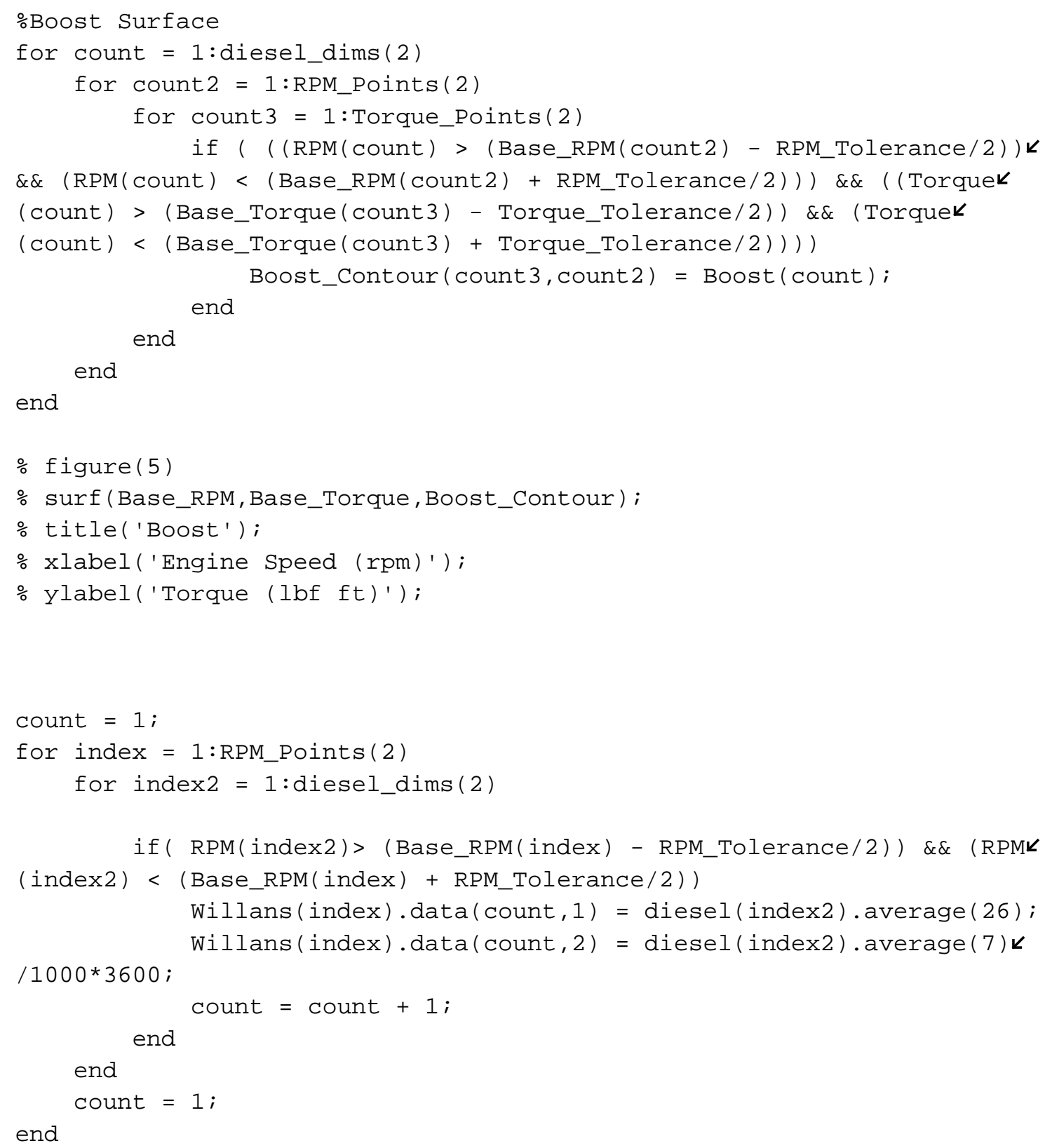




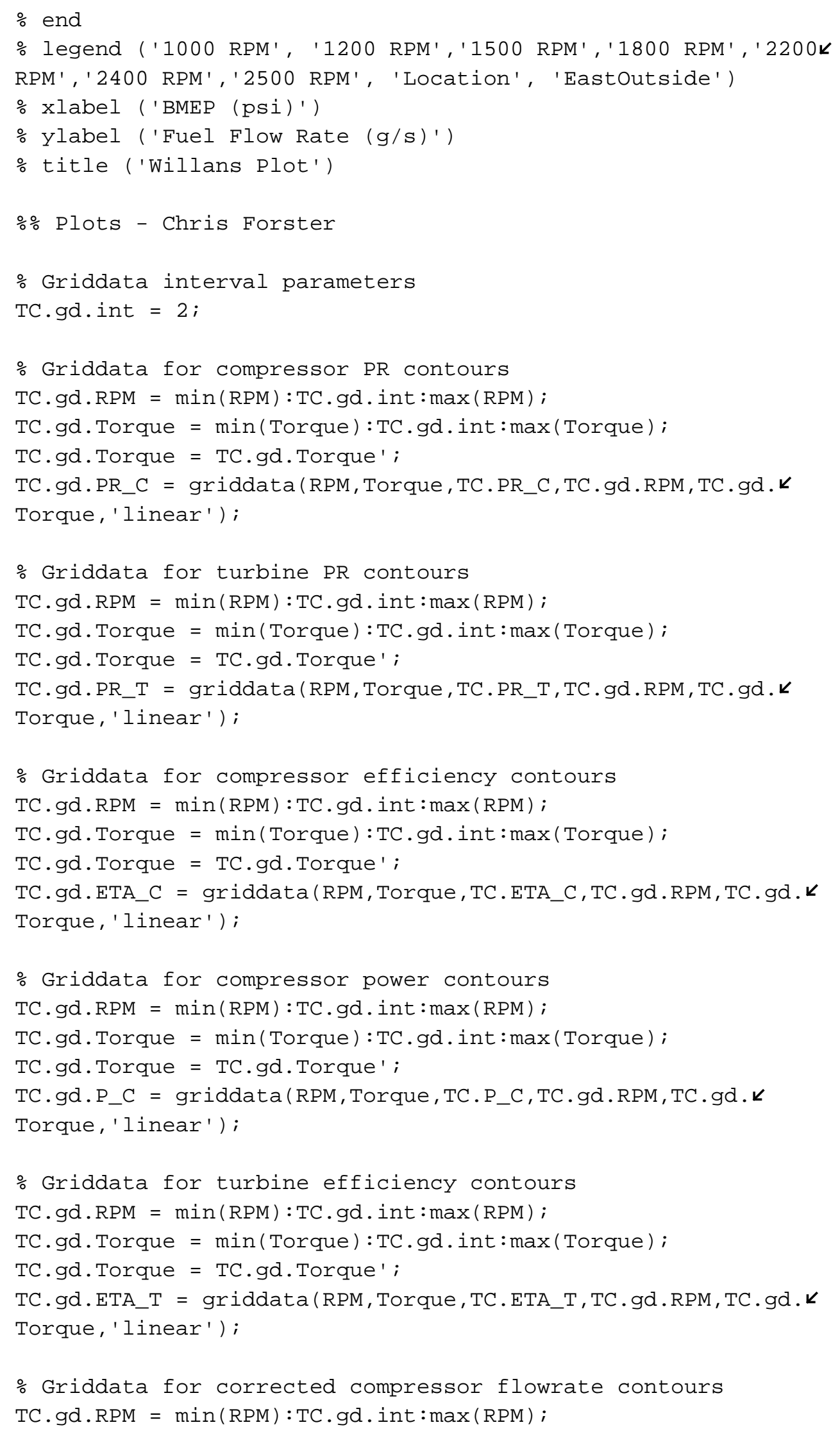




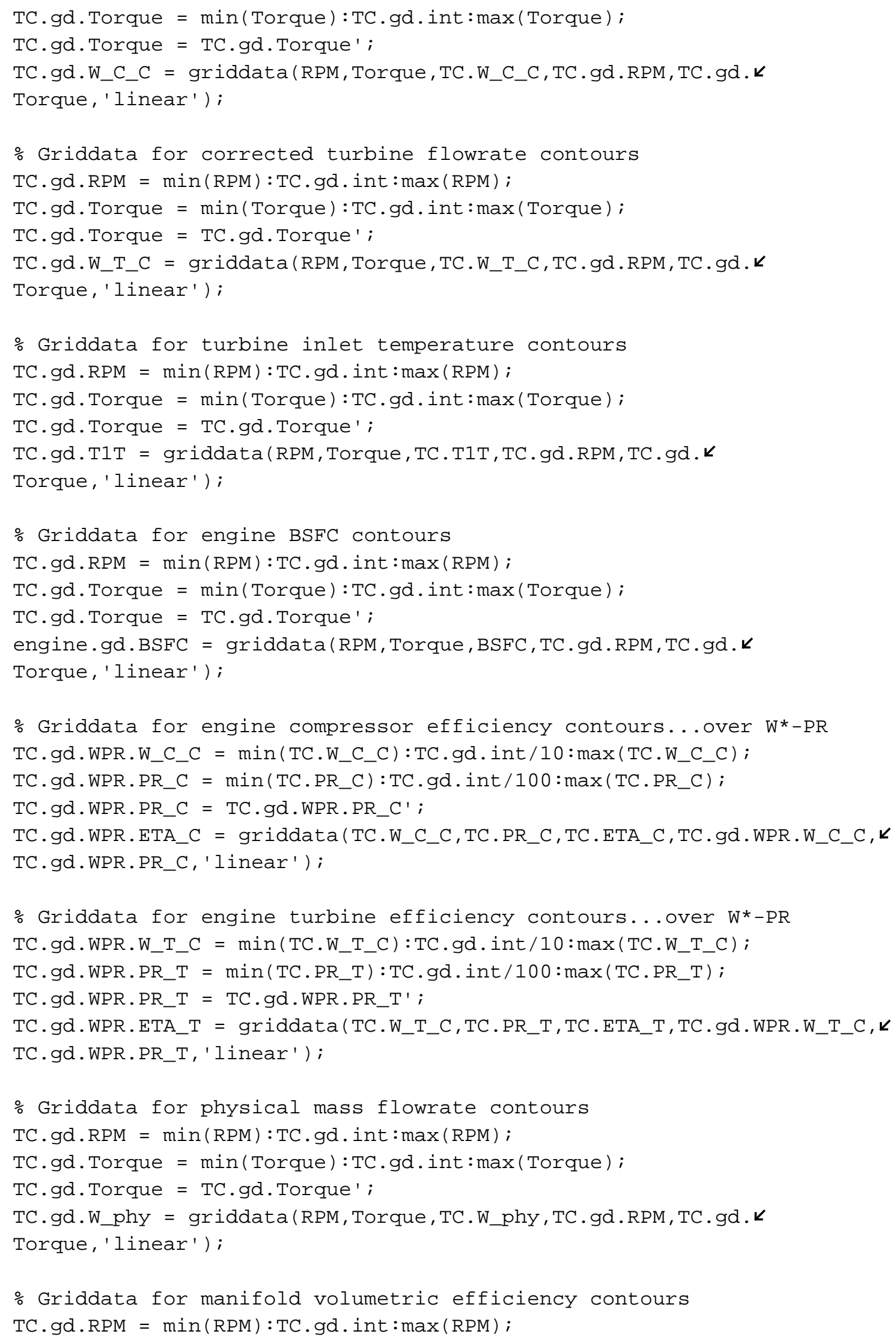




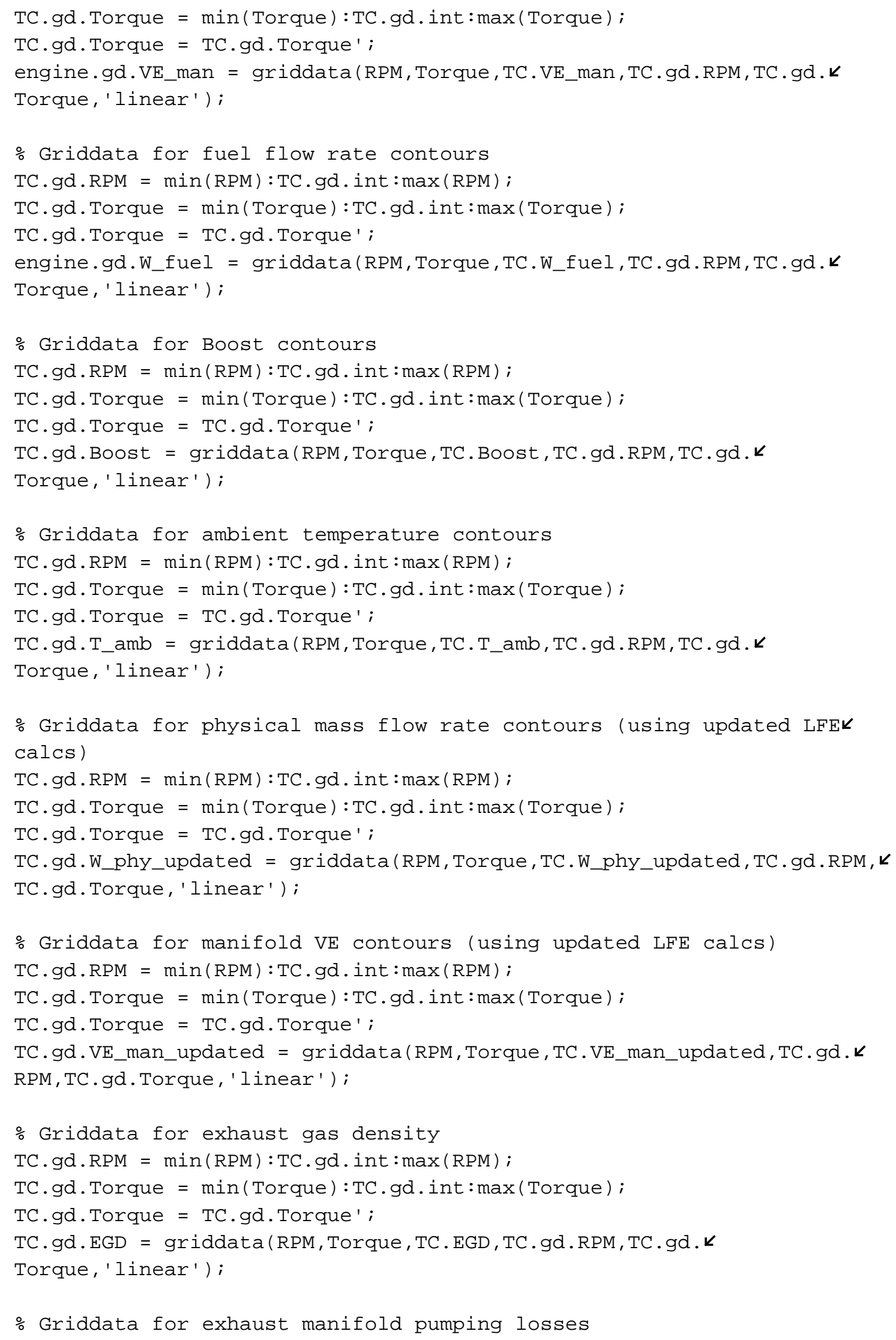




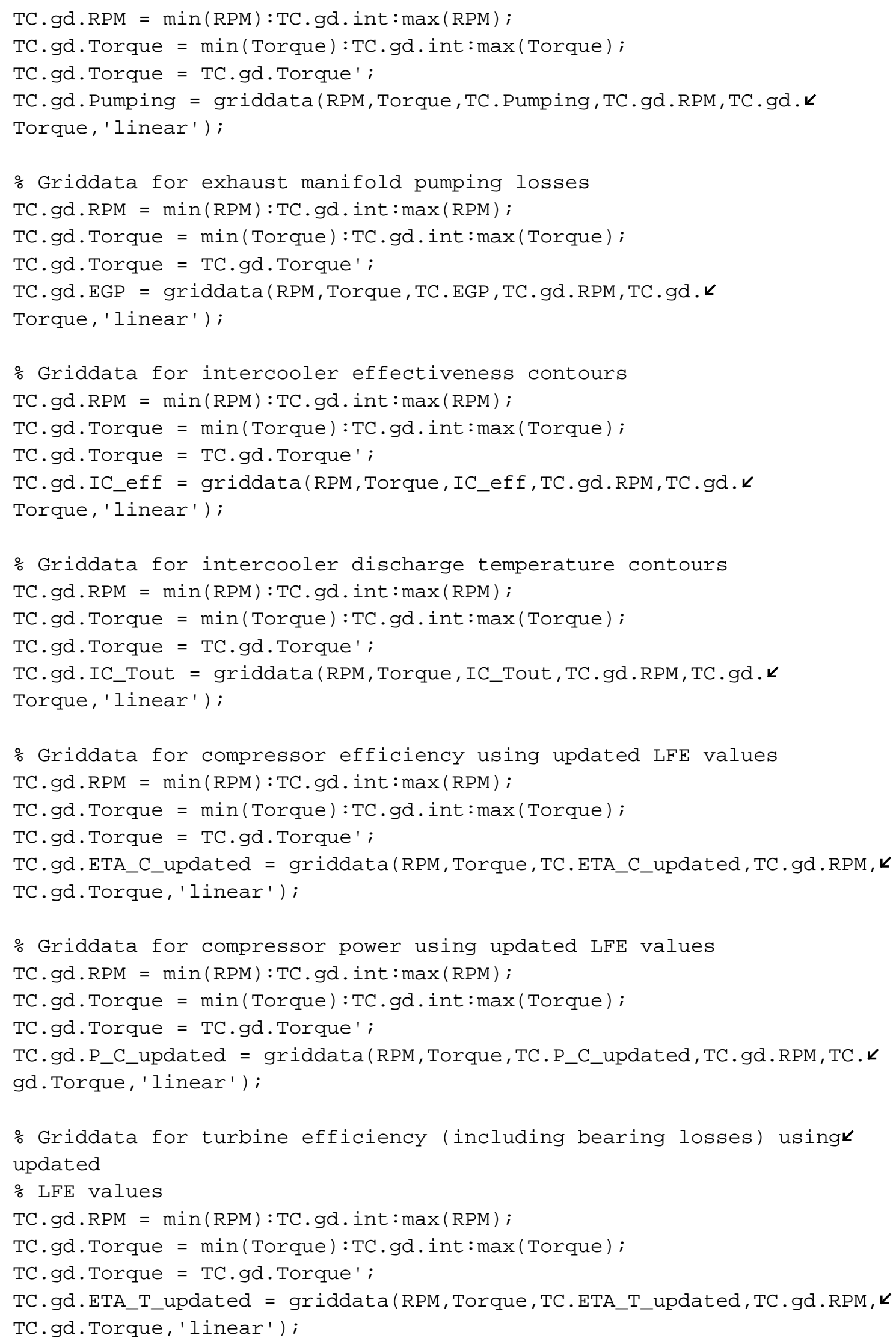


\% Griddata for corrected compressor flow using updated LFE values

TC.gd.RPM = $\min ($ RPM $):$ TC.gd.int $: \max ($ RPM $)$;

TC.gd.Torque $=\min ($ Torque $):$ TC.gd.int $: \max ($ Torque $)$;

TC.gd. Torque = TC.gd. Torque';

TC.gd.W_C_C_updated = griddata(RPM,Torque, TC.W_C_C_updated, TC.gd.RPM, $\boldsymbol{k}$ TC.gd.Torque, 'linear' );

\% Griddata for corrected turbine flow using updated LFE values

TC.gd.RPM $=\min (\mathrm{RPM}): \mathrm{TC} \cdot \mathrm{gd}$. int $: \max (\mathrm{RPM})$;

TC.gd.Torque $=\min ($ Torque $):$ TC.gd.int $: \max ($ Torque $) ;$

TC.gd. Torque $=$ TC.gd. Torque';

TC.gd.W_T_C_updated = griddata(RPM, Torque, TC.W_T_C_updated, TC.gd.RPM, $\boldsymbol{k}$ TC.gd.Torque, 'linear');

\% Griddata for volumetric flowrate using updated LFE values

TC.gd.RPM $=\min (\mathrm{RPM}): \mathrm{TC} \cdot \mathrm{gd} \cdot$ int $: \max (\mathrm{RPM})$;

TC.gd.Torque $=\min ($ Torque $):$ TC.gd.int $: \max ($ Torque $) ;$

TC.gd.Torque = TC.gd. Torque';

TC.gd.Vflow_updated $=$ griddata(RPM,Torque, TC.Vflow_updated, TC.gd.RPM, $k$ TC.gd.Torque, ' linear');

\% Griddata for engine pressure differential

TC.gd.RPM $=\min (\mathrm{RPM}): \mathrm{TC} \cdot \mathrm{gd} \cdot$ int $: \max (\mathrm{RPM})$;

TC.gd.Torque $=\min ($ Torque $):$ TC.gd.int $: \max ($ Torque $)$;

TC.gd.Torque = TC.gd. Torque';

TC.gd.EDP = griddata(RPM, Torque, TC.EDP, TC.gd.RPM, TC.gd. $\boldsymbol{k}$

Torque, 'linear' ');

\% Griddata for pumping losses using engine $d p$

TC.gd.RPM $=\min (\mathrm{RPM}): \mathrm{TC} \cdot \mathrm{gd} \cdot$ int $: \max (\mathrm{RPM})$;

TC.gd. Torque $=\min ($ Torque $):$ TC.gd $\cdot$ int $: \max ($ Torque $) ;$

TC.gd. Torque $=$ TC.gd. Torque';

TC.gd.Pumping_dp $=$ griddata(RPM,Torque, TC.Pumping_dp, TC.gd.RPM, TC.gd.k Torque, 'linear' );

\% Griddata for ACM DAR Cooling Capacity

TC.gd.RPM $=\min ($ RPM $):$ TC.gd.int: $\max ($ RPM $)$;

TC.gd. Torque $=\min ($ Torque $):$ TC.gd $\cdot$ int $: \max ($ Torque $) ;$

TC.gd. Torque = TC.gd. Torque' ;

ACM.gd.DARcap = griddata $($ RPM, Torque, ACM.DARCap, TC.gd.RPM, TC.gd. $\boldsymbol{k}$

Torque, ' linear' );

speed. $\min =1000 ;$

speed. $\max =1600$;

$\mathrm{tq} \cdot \mathrm{min}=50$;

$\mathrm{tq} \cdot \max =300$;

figure(7) 
contourf (TC.gd.RPM, TC.gd. Torque, engine.gd.BSFC, 25)

$x \lim ([$ speed.min speed.max])

$y \lim ([\mathrm{tq} \cdot \min \mathrm{tq} \cdot \max ])$

axis square

grid on

title('Engine BSFC')

xlabel('Engine Speed [RPM]')

ylabel('Engine Load [lbf-ft]')

$\mathrm{h}=\operatorname{colorbar}($ 'vert ');

ylabel(h, '[lbm/hp-hr]')

figure(8)

contourf (TC.gd.RPM, TC.gd. Torque, TC.gd.T1T, 20)

$x \lim ([$ speed.min speed.max])

$y \lim ([\mathrm{tq} \cdot \min \mathrm{tq} \cdot \max ])$

axis square

grid on

title('Engine Exhaust Gas Temperature (EGT)')

xlabel('Engine Speed [RPM]')

ylabel('Engine Load [lbf-ft]')

$\mathrm{h}=$ colorbar ('vert');

ylabel (h, '[deg. F]')

figure(9)

contourf(TC.gd.RPM, TC.gd.Torque, TC.gd.ETA_C, 20)

$x \lim ([$ speed.min speed.max])

$y \lim ([\mathrm{tq} \cdot \min \mathrm{tq} \cdot \max ])$

axis square

grid on

title('TC Compressor Efficiency')

xlabel('Engine Speed [RPM]')

ylabel('Engine Load [lbf-ft]')

$\mathrm{h}=$ colorbar('vert');

ylabel $(h, '[-] ')$

figure (10)

contourf(TC.gd.RPM, TC.gd.Torque, TC.gd.ETA_T, 20)

$x \lim ([$ speed.min speed.max])

$y \lim ([\mathrm{tq} \cdot \min \mathrm{tq} \cdot \max ])$

axis square

grid on

title('TC Turbine Efficiency')

xlabel('Engine speed [RPM]')

ylabel('Engine Load [lbf-ft]')

$\mathrm{h}=\operatorname{colorbar}$ ('vert');

ylabel $(h, '[-] ')$

figure(11)

contourf(TC.gd.WPR.W_C_C,TC.gd.WPR.PR_C,TC.gd.WPR.ETA_C, ( $.40: 0.05: K$ 


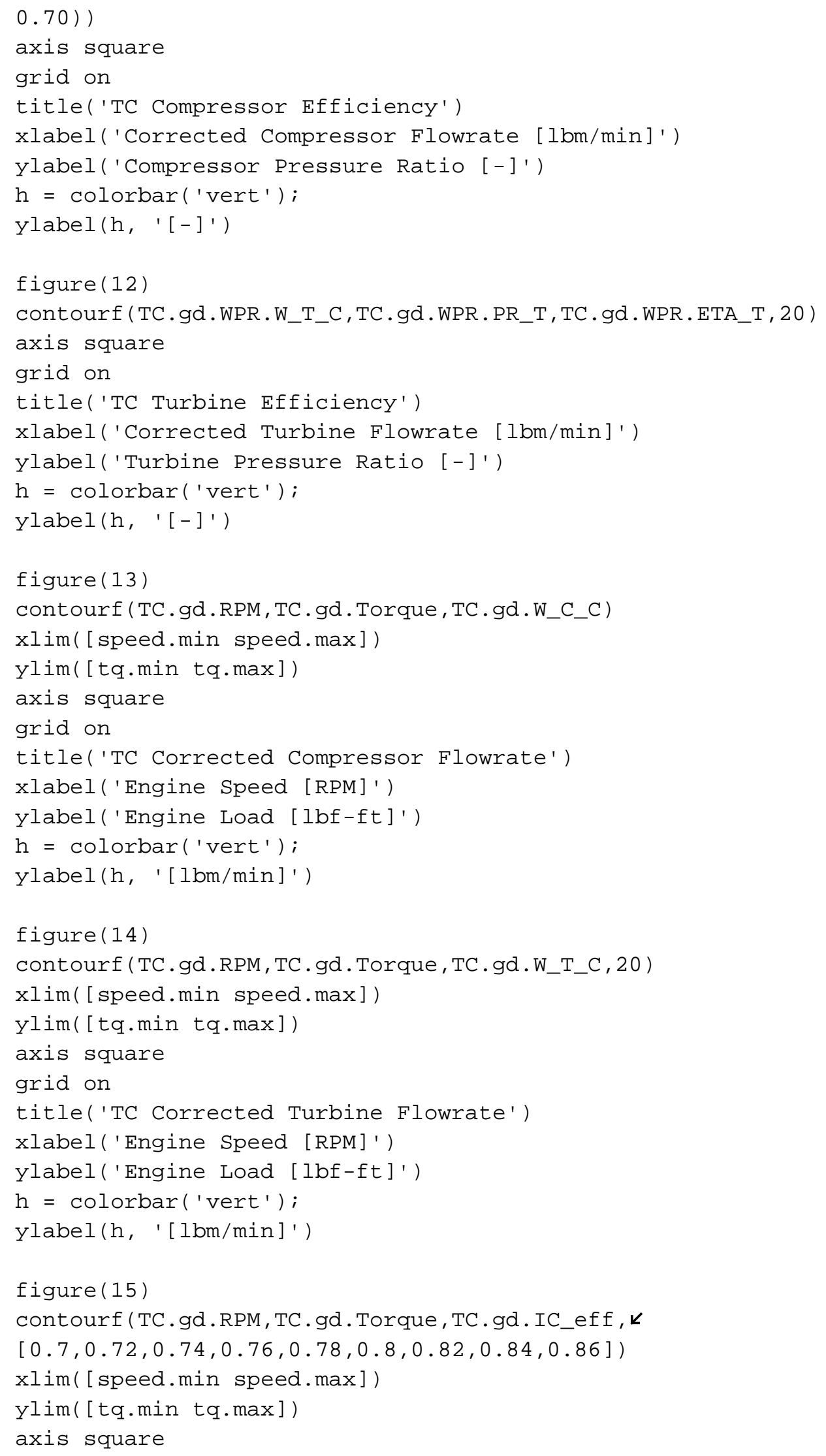




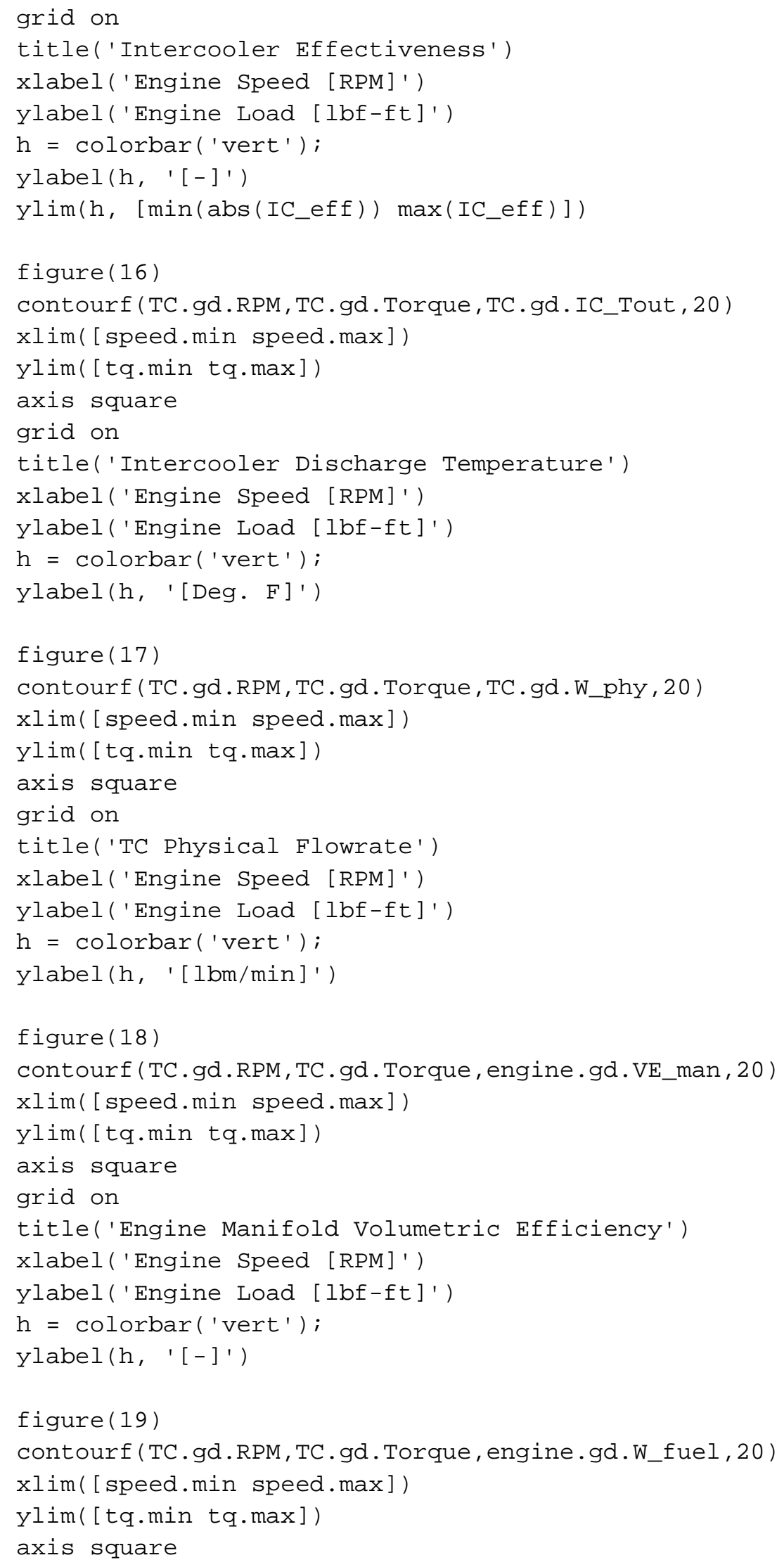




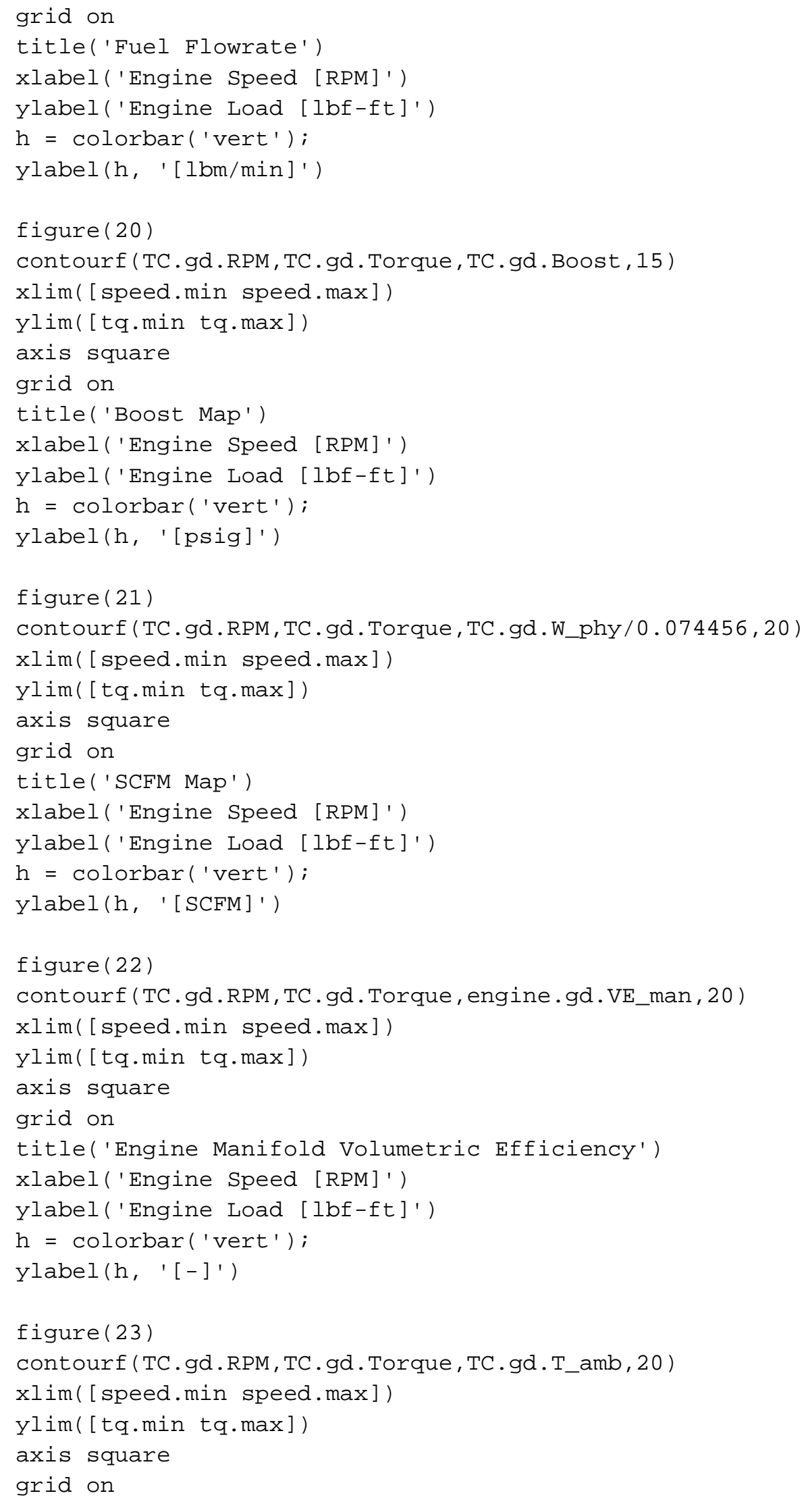




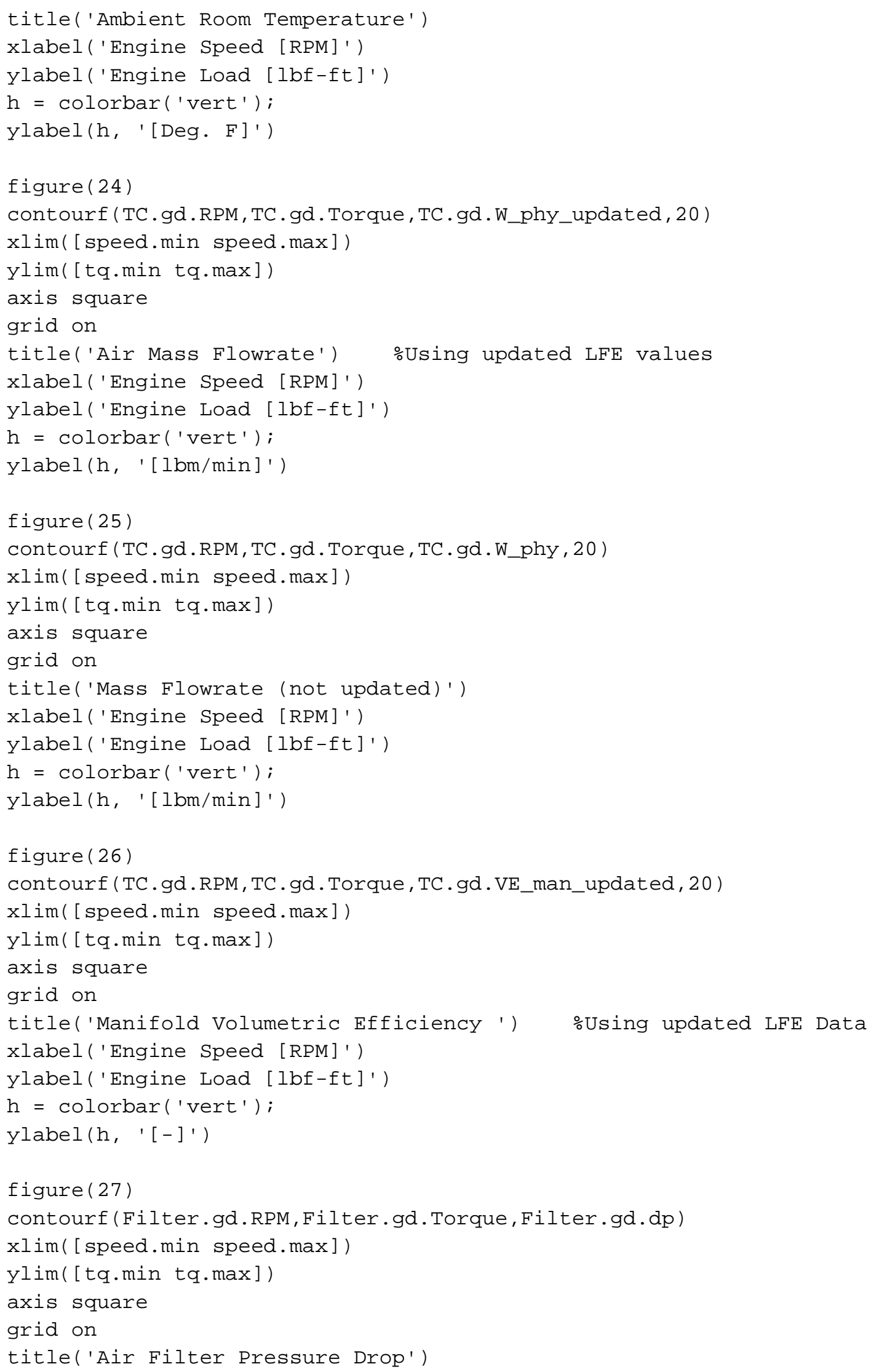




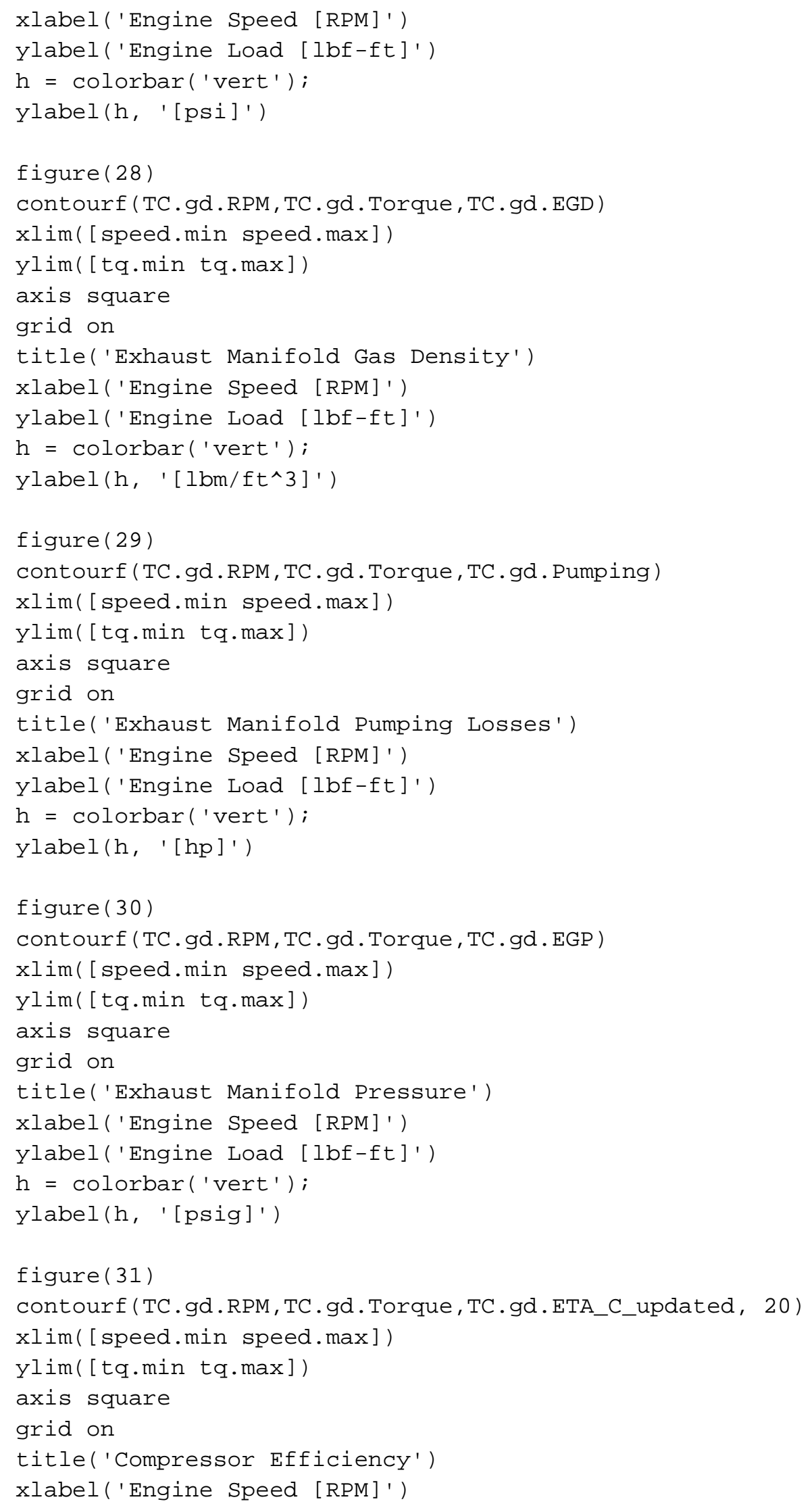




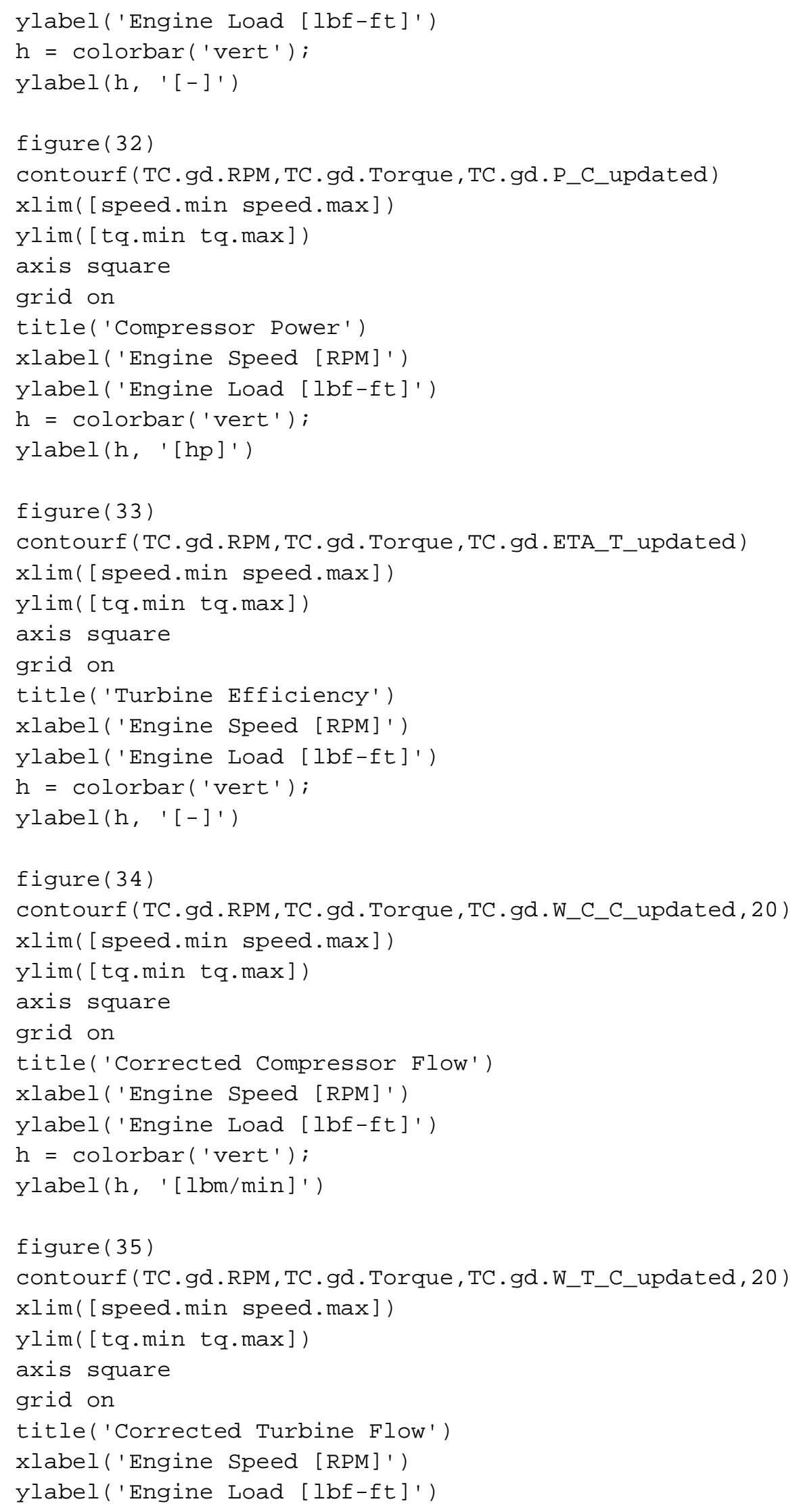




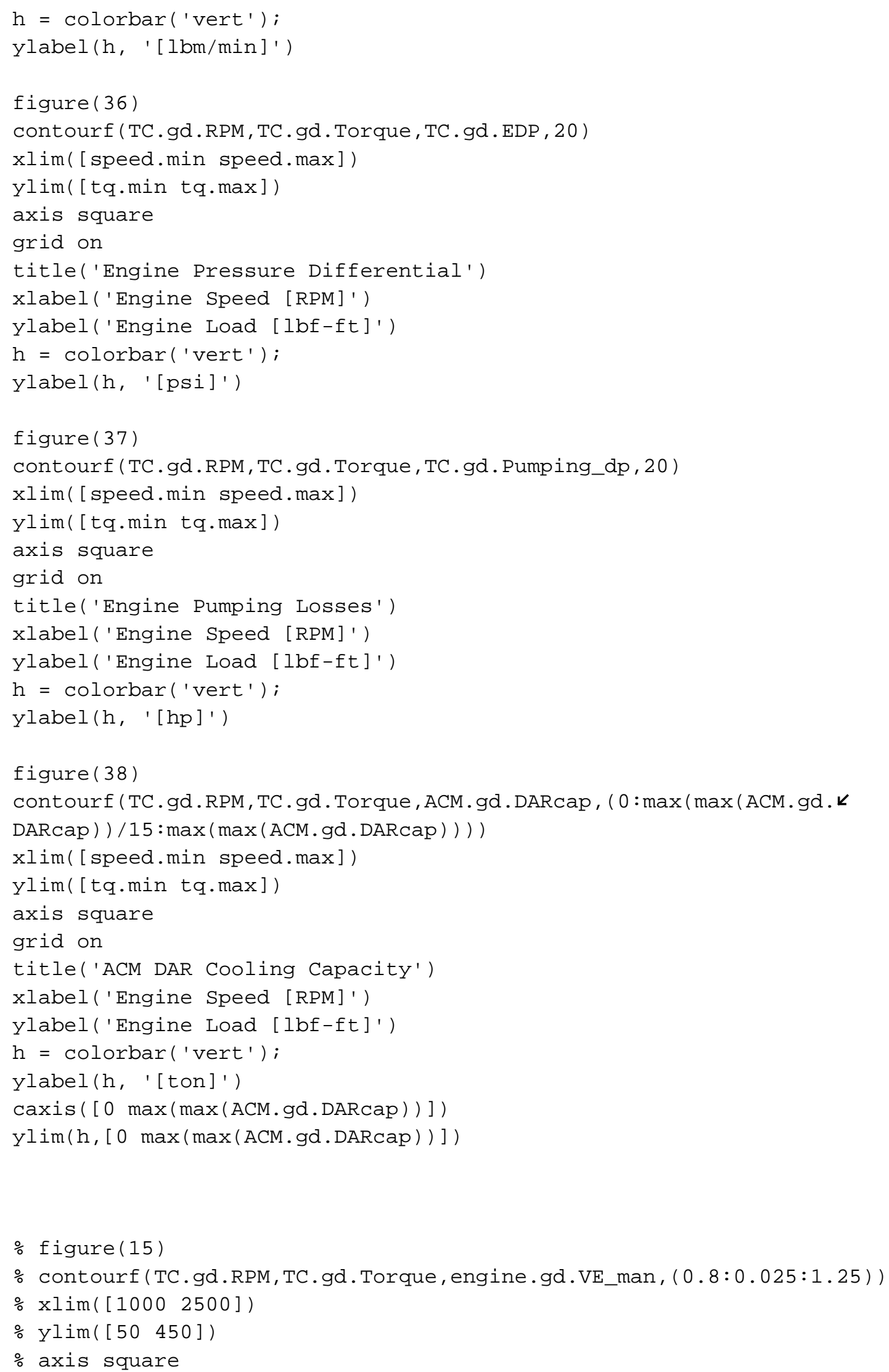




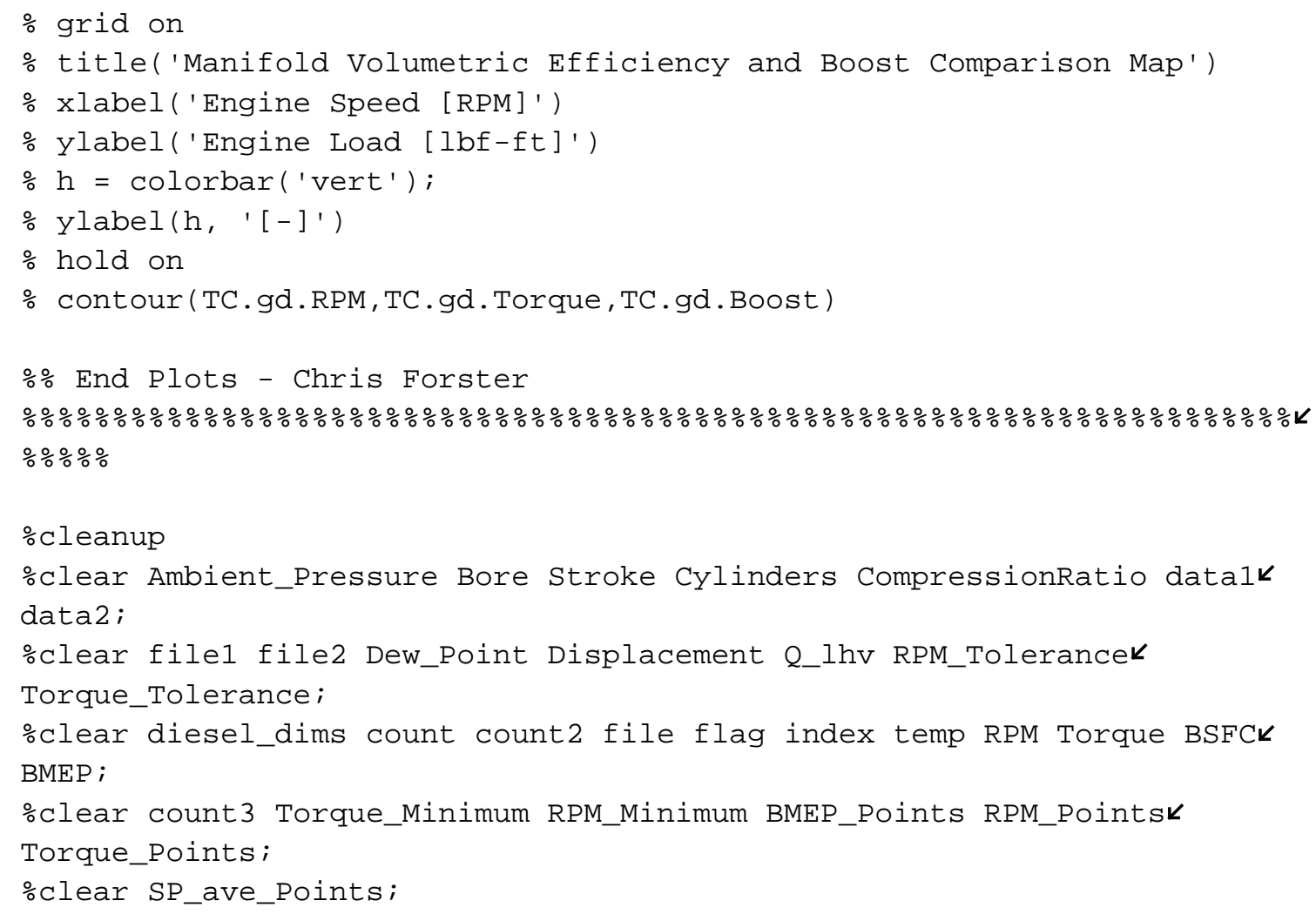


\% Chris Forster

$\%$ 6/20/2009

\% This function is a viscosity correction factor for LFE measurements.

function $[$ up] = viscosity_correction $(T)$

v_col1 $=50: 159$;

v_col2 $=\left[\begin{array}{llllllllll}1.03034 & 1.02877 & 1.02720 & 1.02564 & 1.02408 & 1.02253 & 1.02099 k\end{array}\right.$

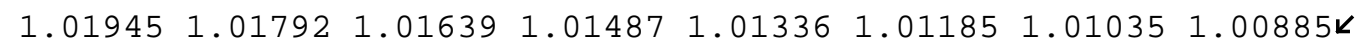

$1.007361 .005881 .004401 .00292 \ldots$

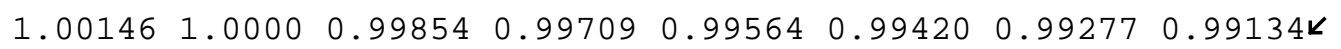

$\begin{array}{lllllllll}0.98992 & \odot .98850 & 0.98709 & 0.98568 & 0.98428 & 0.98288 & 0.98149 & 0.98010 \mathrm{~K}\end{array}$

$0.97872 \quad 0.97734 \quad 0.97597 \quad 0.97461 \quad 0.97325 \quad \ldots$

$\begin{array}{lllllllll}0.97189 & 0.97054 & 0.96919 & 0.96785 & 0.96651 & 0.96518 & 0.96386 & 0.96253 \mathrm{~K}\end{array}$

$\begin{array}{lllllllll}0.96122 & 0.95991 & 0.95860 & 0.95729 & 0.95600 & 0.95470 & 0.95341 & 0.95213 \mathrm{~K}\end{array}$

$0.95085 \quad 0.94957 \quad 0.94830 \quad 0.94704 \quad \ldots$

$0.94578 \quad 0.94452 \quad 0.94327 \quad 0.94202 \quad 0.94077 \quad 0.93953 \quad 0.93830 \quad 0.93707 k$

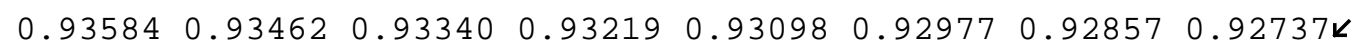

$0.92618 \quad 0.92499 \quad 0.92380 \quad 0.92262 \quad \ldots$

$0.92144 \quad 0.92027 \quad 0.91910 \quad 0.91794 \quad 0.91678 \quad 0.91562 \quad 0.91446 \quad 0.91331 k$

$\begin{array}{llllllll}0.91217 & 0.91103 & 0.90989 & 0.90875 & 0.90762 & 0.90650 & 0.90537 & 0.90425 \mathrm{~K}\end{array}$

$0.903140 .90203 \quad 0.90092 \quad 0.89981 \ldots$

$\begin{array}{lllllllll}0.89871 & 0.89761 & 0.89652 & 0.89543 & 0.89434 & 0.89326 & 0.89218 & 0.89110 \mathrm{~K}\end{array}$ $\odot .89003$ ๑.88896];

v_table $=\left[v_{-}\right.$col1' v_col2'];

up $=$ interp1(v_table $(:, 1), v_{-}$table $\left.(:, 2), T\right)$;

end 
Appendix 0 - Perkins Post-processing Comparison in Matlab 
Chris Forster

clear

clc

close all;

load -mat comparison_data

compare.gd.rpm $=1000: 5: 2500 ;$

compare.gd.torque $=(50: 2: 500)^{\prime}$;

\% Regrid ACM data to common grid for comparison compare.acm.bsfc = interp2(acm.gd.rpm, acm.gd.torque, acm.bsfc, compare.k gd.rpm(ones (1, length (compare.gd.torque)), : ), compare.gd.torque ( : , onesk $(1$, length (compare.gd.rpm $)))$, ' linear' ' NaN) ;

compare. acm. fuel = interp2(acm.gd. rpm, acm.gd.torque, acm. fuel, compare.k gd.rpm(ones (1, length (compare.gd.torque)), : ), compare.gd.torque ( : , onesk $(1$, length (compare.gd.rpm) )), 'linear' 'NaN);

compare.acm.pumping = interp2(acm.gd.rpm,acm.gd.torque, acm.pumping, $\boldsymbol{k}$ compare.gd.rpm(ones $(1$, length (compare.gd.torque) ), : ), compare.gd.torquek $(:$, ones $(1$, length (compare.gd.rpm $)))$, 'linear' 'NaN);

compare. acm.boost $=$ interp2 (acm.gd.rpm, acm.gd.torque, acm.boost, $k$ compare.gd.rpm(ones( 1 , length (compare.gd.torque)), : ), compare.gd.torquek $(:$, ones $(1$, length (compare.gd.rpm $)))$, 'linear', $\mathrm{NaN})$;

compare.acm.DARcap = interp2 (acm.rem.rpm, acm.rem.tq, acm.DARcap, $\boldsymbol{k}$ compare.gd.rpm(ones $(1$, length (compare.gd.torque) ), : ), compare.gd.torquek $(:$, ones $(1$, length (compare.gd.rpm $)))$, 'linear', $\mathrm{NaN})$;

\% Regrid Baseline data to common grid for comparison compare.baseline.bsfc = interp2(baseline.gd.rpm, baseline.gd.torque, $\boldsymbol{k}$ baseline.bsfc, compare.gd.rpm(ones( 1 , length (compare.gd.torque)), : ), $k$ compare.gd.torque ( : , ones ( 1 , length (compare.gd.rpm))), 'linear', NaN); compare. baseline.fuel = interp2 (baseline.gd.rpm, baseline.gd.torque, $k$ baseline.fuel, compare.gd.rpm(ones( 1 , length (compare.gd.torque)), : ), $k$ compare.gd.torque (:, ones $(1$, length (compare.gd.rpm) )), 'linear', NaN); compare.baseline.pumping = interp2(baseline.gd.rpm, baseline.gd.torque, $\boldsymbol{k}$ baseline.pumping, compare.gd.rpm(ones ( 1 , length (compare.gd.torque)) , : ), $k$ compare.gd.torque ( : , ones ( 1 , length (compare.gd.rpm))), 'linear ', NaN); compare. baseline. boost $=$ interp2 (baseline.gd. $r p m$, baseline.gd.torque, $\boldsymbol{k}$ baseline.boost, compare.gd.rpm(ones( 1 , length (compare.gd.torque)), : ), $k$ compare.gd.torque (:, ones (1, length (compare.gd.rpm)) ), 'linear', NaN); compare.baseline.VE_man = interp2 (baseline.gd.rpm, baseline.gd.torque, $\boldsymbol{k}$ baseline.VE_man, compare.gd.rpm(ones( 1 , length (compare.gd.torque) ), : ), $k$ compare.gd.torque ( : , ones ( 1 , length (compare.gd.rpm)) ), 'linear', NaN);

\% Regrid stock turbocharger data to common grid for comparison compare.stock.VE_man = interp2(stock.gd.rpm, stock.gd.torque, stock. $k$ VE_man, compare.gd.rpm(ones(1, length (compare.gd.torque)), : ), compare.gd.k torque ( $:$, ones $(1$, length (compare.gd.rpm $)))$, 'linear', NaN); 
compare.stock.bsfc = interp2(stock.gd.rpm,stock.gd.torque, stock.bsfc, $\boldsymbol{k}$ compare.gd.rpm (ones (1, length (compare.gd.torque)), :), compare.gd.torquek $(:$, ones $(1$, length (compare.gd.rpm) )), 'linear', NaN) ;

\% Comparing difference in pumping losses

compare.diff.pumping = compare.acm.pumping - compare.baseline.pumping;

\% Calculating power based on difference

compare.diff.bsfcpower = (compare.acm.fuel - compare.baseline.fuel). $\boldsymbol{k}$

/compare.baseline.bsfc*60;

- Calculate percent difference in methods

compare.diff.method $=$ (compare.diff.pumping - compare.diff.bsfcpower). $\boldsymbol{k}$

/compare.diff.bsfcpower*100;

\% Compare difference in boost

compare.diff.boost $=$ compare.acm.boost - compare.baseline.boost;

- Calculate COP based on BSFC differences

compare.acm. DARcop1 = compare.acm.DARcap*4.71617735./compare.diff.k

bsfcpower;

\% Calculate COP based on BSFC differences

compare.acm.DARcop2 = compare.acm.DARcap*4.71617735./compare.diff.k

pumping;

\% Compare stock turbo and GT VE_man data (percent difference)

compare.diffstock.VE_man = (compare.baseline.VE_man - compare.stock.k

VE_man)./compare.stock.VE_man*100;

\% Compare stock turbo and GT VE_man data (difference only)

compare.diffstock.VE_man2 = (compare.baseline.VE_man - compare.stock.k VE_man);

o Compare stock turbo and GT bsfC data

compare.diffstock.bsfc $=$ (compare.baseline.bsfc - compare.stock.bsfc).k

/compare.stock.bsfc*100;

\% Arrange boost-percent difference in method data into a vector for $2 \mathrm{~d} \boldsymbol{k}$ plot

compare.diff.boostsize = size(compare.diff.boost);

compare.diff.methodsize = size(compare.diff.method);

compare.twod.boostlength = compare.diff.boostsize $(1) *$ compare.diff.k

boostsize(2);

compare.twod.methodlength $=$ compare.diff.methodsize $(1){ }^{*}$ compare.diff.k

methodsize(2);

compare.twod.boost $=$ reshape (compare.diff.boost, compare.twod.k

boostlength, 1); 
compare.twod.method = reshape (compare.diff. method, compare.twod. $\boldsymbol{k}$ methodlength, 1);

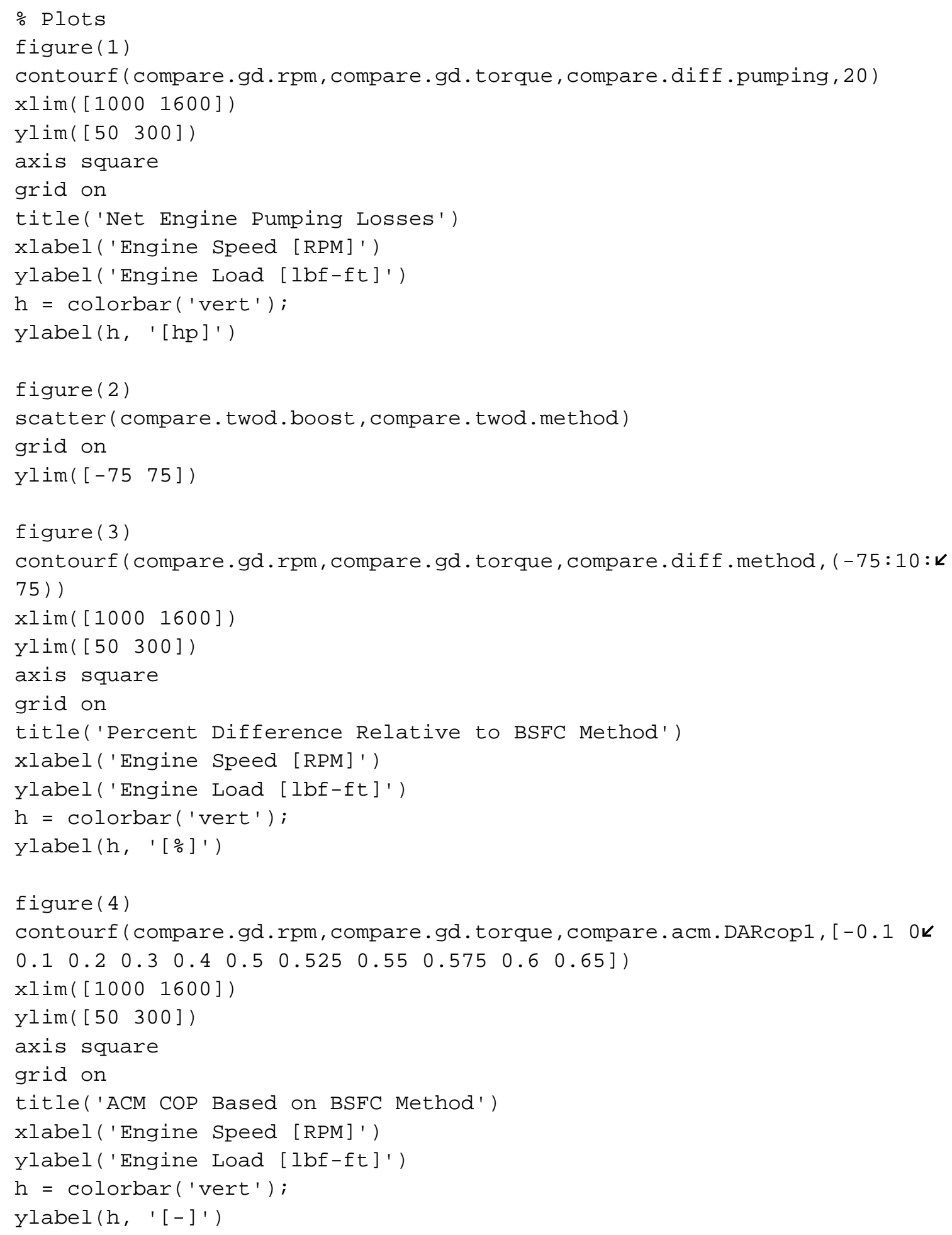




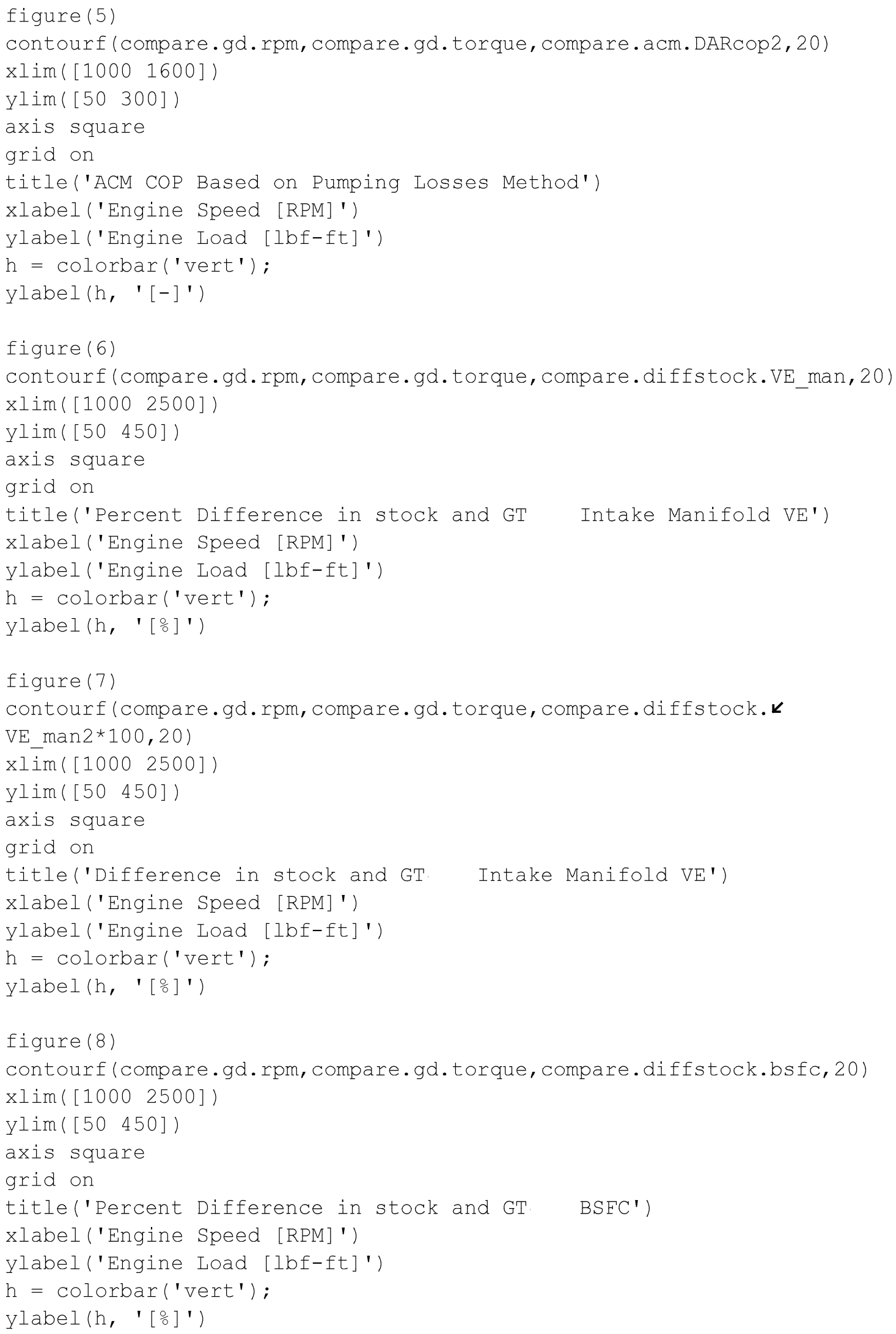




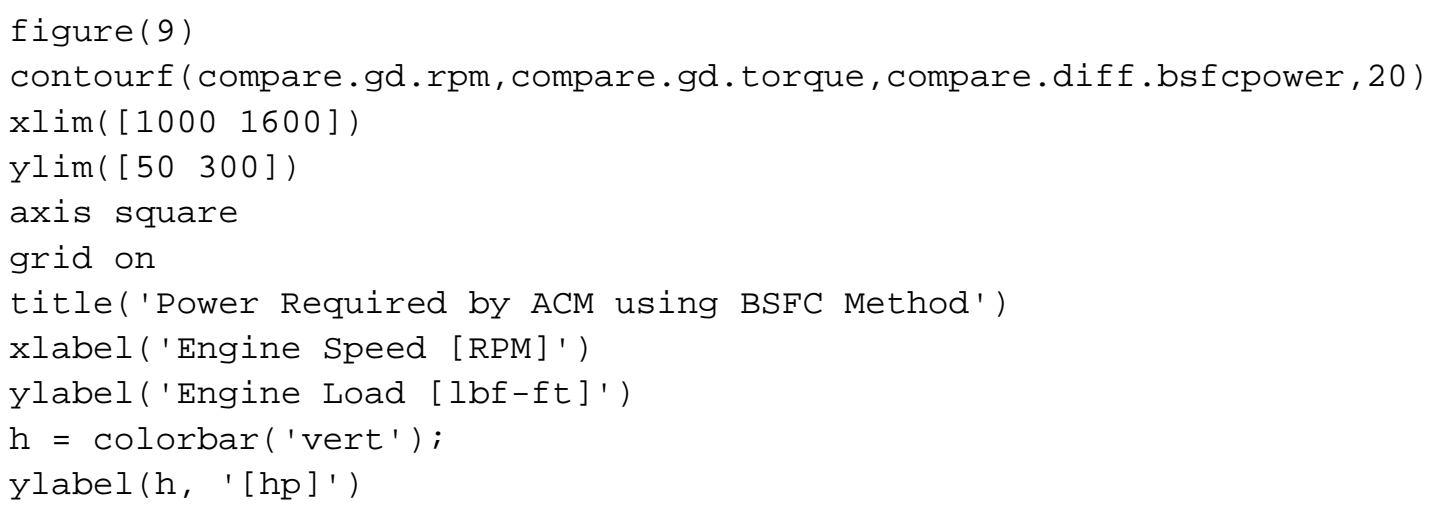

